\title{
Rußbildung in der Kohlenwasserstoffpyrolyse hinter Stoßwellen
}

\author{
Dissertation \\ zur Erlangung des Doktorgrades \\ der Mathematisch-Naturwissenschaftlichen Fachbereiche \\ der Georg-August-Universität zu Göttingen
}

\author{
vorgelegt von \\ Dietmar Tanke \\ aus Walsrode
}

Göttingen 1994 
Referent: Prof. Dr. H. Gg. Wagner

Korreferent: Prof. Dr. Drs. E.h., h.c. O. Glemser

Tag der mündlichen Prüfung: 24. Januar 1995 


\section{Inhaltsverzeichnis}

1 Einleitung 1

2 Experimentelle Methoden 2

2.1 Die Stoßrohrmethode . . . . . . . . . . . . . . . . . . . . 2

2.1.1 Qualitative Theorie des einfachen Stoßwellenrohres . . . . . . . . 3

2.1.2 Quantitative Theorie des einfachen Stoßwellenrohres . . . . . . . 4

2.2 Die Stoßwellenapparatur . . . . . . . . . . . . . . . . 6

2.2 .1 Membran .................... 7

2.3 Optischer Aufbau . . . . . . . . . . . . . . . . . . 7

2.3.1 Signalerfassung und EDV . . . . . . . . . . . . . . 7

2.4 Absorption von Licht durch Rußpartikel . . . . . . . . . . . . . . . 8

2.5 Probenentnahme und Gasanalyse . . . . . . . . . . . . . . . . . . 9

2.5 .1 Ventil . . . . . . . . . . . . . . . . 9

2.5.2 Gaschromatographische Analyse . . . . . . . . . . . . . 11

2.5.3 Elektronenmikroskopie . . . . . . . . . . . . . . 12

2.5 .4 Gas-Adsorption . . . . . . . . . . . . . . . 13

2.5 .5 HPLC . . . . . . . . . . . . . . . 13

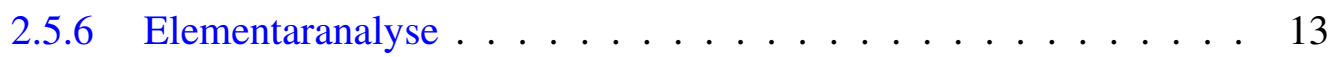

2.6 Chemikalien und Gase . . . . . . . . . . . . . . . . . . . . . . . . . . . . . . . . . . . . . . . 13

2.7 Versuchsausführung . . . . . . . . . . . . . . . . . . . 14

3 Darstellung der Meßergebnisse $\quad 15$

3.1 Absorption- und Rußausbeute-Zeitprofil . . . . . . . . . . . . . . . . . . . 15

3.2 Induktionszeit . . . . . . . . . . . . . . . . . . . . 17

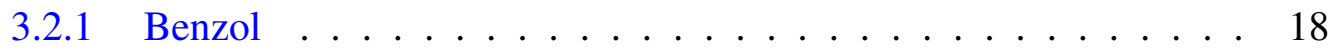

3.2 .2 Phenylacetylen . . . . . . . . . . . . . . 20

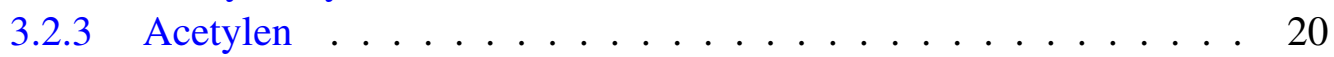

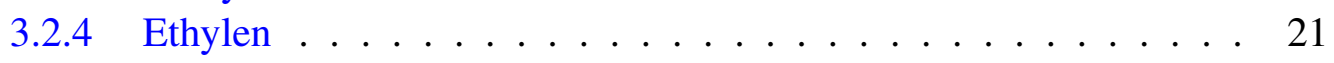

3.2 .5 Methan ....................... 21

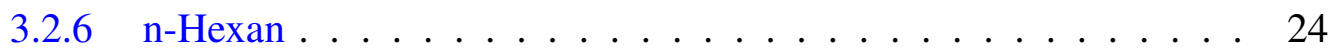

3.2.7 Vergleich der Kohlenwasserstoffe . . . . . . . . . . . . . . 24

3.2 .8 Messungen mit der Laserdiode . . . . . . . . . . . . . . . . . . 26

3.3 Rußmassenwachstum . . . . . . . . . . . . . . . . . 28

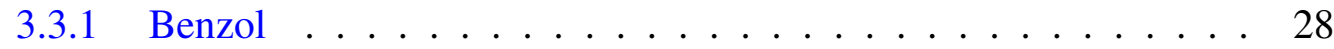

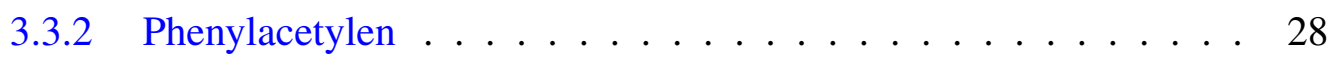

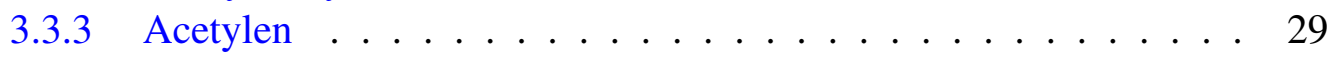

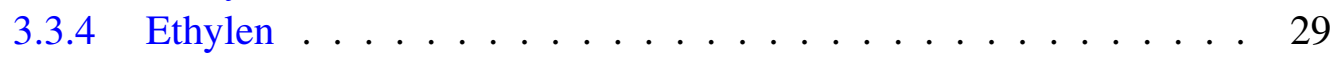

3.3 .5 Methan ........................ 30 
3.3 .6 n-Hexan . . . . . . . . . . . . . . . 30

3.3.7 Vergleich der Kohlenwasserstoffe . . . . . . . . . . . . . 34

3.4 Rußausbeute . . . . . . . . . . . . . . . . . . . . . 34

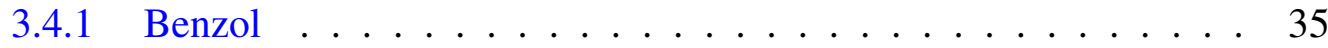

3.4 .2 Phenylacetylen . . . . . . . . . . . . . 36

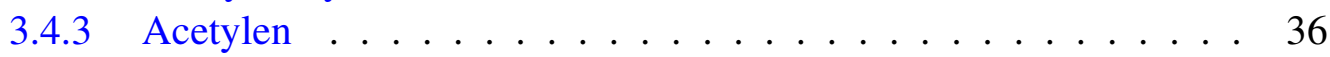

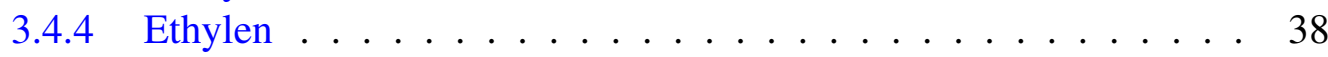

3.4 .5 Methan ........................... 38

3.4 .6 n-Hexan ............................ 40

3.4.7 Vergleich der Kohlenwasserstoffe . . . . . . . . . . . . . 40

3.4.8 Einfluß der Kohlenstoffkonzentration . . . . . . . . . . . . . 42

3.5 Additiv Eisenpentacarbonyl . . . . . . . . . . . . . . . . . . . 42

3.6 Gasförmige Endprodukte . . . . . . . . . . . . . . . . . . 44

3.6.1 Methan-Ausbeute . . . . . . . . . . . . . . . 44

3.6.2 Acetylen-Ausbeute . . . . . . . . . . . . . 46

3.6.3 Ethylen-Ausbeute . . . . . . . . . . . . . . . . 46

3.6 .4 Ethan-Ausbeute . . . . . . . . . . . . . . . 46

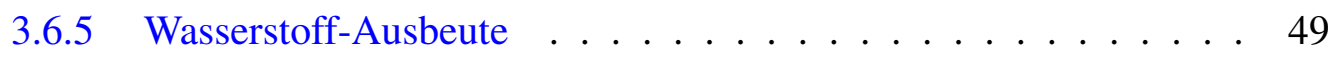

3.6.6 Höhere Kohlenwasserstoffe . . . . . . . . . . . . . . . . . 49

3.6.7 Wasserstoff-Massenbilanz . . . . . . . . . . . . . . . 49

3.6.8 Kohlenstoff-Massenbilanz ........................ 51

3.6.9 Einfluß des Gesamtdruckes . . . . . . . . . . . . . . . . . . . . . 51

3.6.10 Polycyclische Aromaten . . . . . . . . . . . . . . . 52

3.7 Elementaranalyse . . . . . . . . . . . . . . . 52

3.8 Rußpartikelstruktur . . . . . . . . . . . . . . . . . . 54

3.8.1 Spezifische Oberfläche von Ruß . . . . . . . . . . . . . . 54

3.8.2 Elektronenmikroskopie ............... 54

4 Diskussion $\mathbf{5 7}$

4.1 Rußpartikeleigenschaften . . . . . . . . . . . . . . . . . . 57

4.1.1 Durchmesser . . . . . . . . . . . . . . . 57

4.1.2 Rußpartikelanzahldichte . . . . . . . . . . . . 58

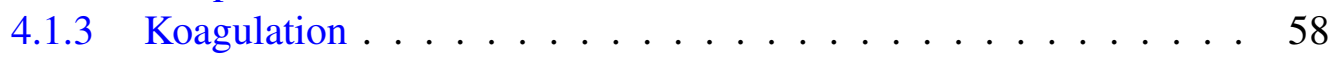

4.2 Einfluß der Lichtwellenlänge auf die detektierte Rußausbeute . . . . . . . . 59

4.3 Modell zur Temperaturabhängigkeit der Rußausbeute . . . . . . . . . . . . 60

4.4 Gasförmige Pyrolyseprodukte . . . . . . . . . . . . . . . . 61

4.5 Induktionszeit . . . . . . . . . . . . . . . . . . . 66

4.6 Vergleich mit vorgemischten Flammen . . . . . . . . . . . . . . . . 67

4.6.1 Rußmassenwachstumskonstante ............... . . 67

4.6.2 Rußausbeute . . . . . . . . . . . . . . . . . . 69

4.7 Einfluß von Eisenpentacarbonyl . . . . . . . . . . . . . . . . 70

5 Anhang $\quad \mathbf{7 5}$

5.1 Logarithmisch normale Verteilungsfunktion . . . . . . . . . . . . . 75

5.2 Symbolverzeichnis . . . . . . . . . . . . . . . 76

5.3 Berechnung von Stoßwellenparametern . . . . . . . . . . . . . 77

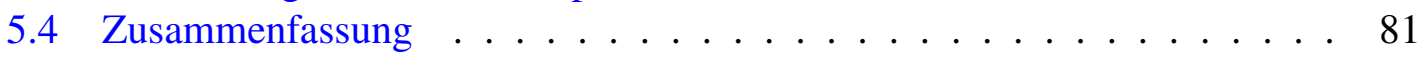

5.5 Danksagung und Lebenslauf . . . . . . . . . . . . . . . . . . . 82 


\section{Kapitel 1}

\section{Einleitung}

Ruß ist seit Jahrhunderten als Produkt von Verbrennungen und Pyrolysen bekannt. In der chemischen Industrie werden jährlich weltweit mehrere Millionen Tonnen Ruß industriell in verschiedenen großtechnischen Verfahren hergestellt. Als Werkstoff findet Ruß Einsatz als Füll- und Stützstoff in der Gummiindustrie. In der Farben- und Kunststoffindustrie wird Ruß als Schwarzpigment eingesetzt.

Wegen seiner guten Strahlungseigenschaften ist Ruß in Großfeuerungsanlagen zur Strahlungswärmeübertragung ein erwünschtes Zwischenprodukt, als Abgasbestandteil jedoch führt er zu einer Belastung der Umwelt.

Aus ökologischer wie ökonomischer Notwendigkeit ist man deshalb seit einigen Jahren intensiv bemüht, die Rußpartikelemissionen von Verbrennungsanlagen, und insbesondere von Kraftfahrzeugmotoren zu reduzieren. Dieses setzt eine vertiefte Kenntnis der zur Rußbildung führenden physikalisch-chemischen Reaktionsmechanismen voraus. Bei der Untersuchung dieser Mechanismen ist zu berücksichtigten, daß in modernen Dieselmotoren unmittelbar nach der Zündung für einige hundert Mikrosekunden sehr hohe Temperaturen $(\approx 2000 \mathrm{~K})$, Drücke $(\approx 5 \mathrm{MPa})$ und Kohlenstoffdichten $\left(\approx 20 \mathrm{~mol} / \mathrm{m}^{3}\right)$ herrschen.

Die Aufklärung von Rußbildungsmechanismen ist möglich durch Untersuchungen in Strömungsreaktoren, in Flammenexperimenten und durch Kohlenwasserstoffpyrolysen in Stoßrohren. Über rußende Flammen ist eine große Vielfalt an Literatur veröffentlicht worden. Aus den vorliegenden Daten war es aber bisher nicht möglich ein allgemeingültiges Modell der Rußbildung aufzustellen. Deshalb besteht großes Interesse, die Rußbildung unter genau definierten Bedingungen in einem möglichst einfachen System, d. h. bei konstanten Zustandsgrößen und mit wenigen Edukten (insbesondere sauerstofffrei) zu beobachten.

Die Stoßrohrmethode bietet die Möglichkeit die Temperatur, den Druck und die Testgaszusammensetzung einerseits genau zu bestimmen und andererseits diese Parameter in weiten Grenzen relativ unabhängig zu variieren. Für eine Reihe von ungesättigten Kohlenwasserstoffen ist die Rußbildung in Stoßwellenexperimenten bis zu einem Druck von $1 \mathrm{MPa}$ untersucht worden. Dabei wurden charakteristische Abhängigkeiten der Rußbildung von Temperatur, Kohlenstoffdichte und Struktur des Kohlenwasserstoffes festgestellt $[1,2,3]$. Untersuchungen für Drücke über $1 \mathrm{MPa}$ liegen nur für wenige Brennstoffe vor $[4,5]$.

Ziel dieser Arbeit war es, simultan Absorptionsmessungen, Gasanalysen und Messungen der finalen Rußpartikeldurchmesser in der Pyrolyse verschiedener Kohlenwasserstoffe auszuführen. Dazu wurden die Temperatur im Bereich von 1500 bis $2500 \mathrm{~K}$ der Druck von 0,5 bis $25 \mathrm{MPa}$ und die Kohlenstoffkonzentration von 0,4 bis $40 \mathrm{~mol} / \mathrm{m}^{3}$ variiert. 


\section{Kapitel 2}

\section{Experimentelle Methoden}

\subsection{Die Stoßrohrmethode}

Als Stoßrohrmethode bezeichnet man ein Meßverfahren bei dem Stoßwellen in einem zylinderförmigen Rohr durch ein Testgas laufen, das dadurch in sehr kurzer Zeit beschleunigt, komprimiert und erhitzt wird. Zur Untersuchung von Gasreaktionen bei höheren Temperaturen $(1000<T / \mathrm{K}<4000)$ ist die Stoßwellenmethode besonders gut geeignet. Dabei werden folgende vorteilhafte Eigenschaften der Stoßwellen ausgenutzt:

- Die Zeit zum Aufheizen und Komprimieren des Testgases $\left(t_{\mathrm{Heiz}} \ll 1 \mu \mathrm{s}\right)$ ist sehr kurz gegenüber der zu erwartenden Reaktionszeit $\left(t_{\text {Reak }} \approx 1 \mathrm{~ms}\right)$.

- Die Zustandsgrößen Druck und Temperatur des Testgases lassen sich in weiten Grenzen relativ unabhängig voneinander variieren und bleiben während des Experiments hinreichend konstant $(\Delta P= \pm 2 \%)$.

- Die Molenbrüche der Komponenten des zu untersuchenden Testgases können um Größenordnungen variiert werden $\left(1<x_{\mathrm{Gas}} / \mathrm{ppm}<50000\right)$, ohne daß die Reaktionsenthalpie merklichen Einfluß auf die Zustandsgrößen des Testgases und damit auf die Stoßwellen hat.

- Bei Stoßrohren mit hinreichend großen Durchmessern können Wandreaktionen vernachlässigt werden. Das in dieser Arbeit verwendete Stoßrohr hat mit $d=70,0 \mathrm{~mm}$ einen genügend großen Innendurchmesser.

- Die Experimente sind gut reproduzierbar. Im Rahmen dieser Arbeit wurden Experimente unter gleichen Bedingungen wiederholt, die im Abstand von Monaten bzw. Jahren durchgeführt wurden. Die erzielten Ergebnisse sind innerhalb der Fehlergrenzen identisch.

Die Einsatzmöglichkeiten der Stoßwellenmethode werden durch folgende Bedingungen eingeschränkt:

- Die maximale Dauer eines Experimentes beträgt für ein konventionelles Stoßrohr von ca. 10 Meter Länge nur wenige Millisekunden $\left(t_{\exp }<3 \mathrm{~ms}\right)$.

- Die zu untersuchende Reaktion findet stets in einem Wärme-Badgas (z. B. Argon) statt, dessen Molenbruch $x_{\text {Badgas }}$ jedoch mindestens $95 \%$ betragen sollte. 
- Stoßwellen mit optimalen Eigenschaften können am besten in einem kreisrunden Rohr mit glatter Oberfläche erzeugt werden. Beim Einbau von Detektoren, Beobachtungsfenstern etc. treten zwangsläufig Abweichungen von dieser optimalen Form auf. Das kann störende sekundäre Stoß- und/oder Expansionswellen zur Folge haben, die jedoch gering gehalten werden können. In dem hier benutzten Stoßrohr sind diese Störungen kleiner als das Signal/Rauschverhältnis des Druck-Zeitprofils, siehe auch Abbildung (3.1).

\subsubsection{Qualitative Theorie des einfachen Stoßwellenrohres}

Auf die Wirkungsweise eines einfachen Stoßrohres wird hier nur schematisch eingegangen. Detaillierte Darstellungen sind in den Standardwerken von [6, 7, 8] beschrieben. Die einfache Stoßwellenapparatur besteht aus einem vakuum- und druckfesten zylindrischen Rohr, das von flachen Endplatten senkrecht zum Rohr abgeschlossen wird und von einer gasundurchlässigen Membran in zwei Teile im Verhältnis von ca. (2:3) getrennt wird. In den längeren Rohrteil, der sogenannte Niederdruckteil (ND), wird das Testgas (Argon) bis zum Druck $P_{1}$ eingelassen. Kurz vor dem Ende des ND-Teils befinden sich Fenster und die Meßoptik. In den kürzeren Rohrteil, den Hochdruckteil (HD), wird bis zum Bersten der Membran das Treibgas (Wasserstoff) mit dem Druck $P_{5}$ eingelassen. Der Berstdruck $P_{5}$ wird durch die Stärke der Membran bestimmt. In den Experimenten dieser Arbeit wurde mit stark unterschiedlichen Druckverhältnissen $\left(P_{5} / P_{1}\right)$ gearbeitet: $\left(P_{5} / P_{1}\right) \approx 12$ bis 250 . Durch Variation dieses Druckverhältnisses kann die gewünschte Temperatur eingestellt werden. Ein höheres Druckverhältnis erzeugt höhere Temperaturen.

Nach dem Bersten der Membran werden Stoßwellen erzeugt, die in Richtung ND-Teil laufen. Gleichzeitig laufen Expansionswellen (Verdünnungswellen) in den HD-Teil. Sie sind für das weitere Geschehen im Stoßrohr zunächst nicht weiter von Interesse. Die Stoßwellen jedoch addieren sich nach einer Länge von einigen Stoßrohrdurchmessern zu einer dünnen ebenen Stoßfront (einfallende Stoßwelle) von der Dicke in der Größenordnung der freien Weglänge des Gases. Diese Stoßfront durchläuft mit Überschallgeschwindigkeit (z. B. 3 MACH bezogen auf das kalte Testgas) den ND-Teil und erhöht schlagartig die Zustandsgrößen des Testgases von $\left(T_{1}, \rho_{1}\right)$ auf $\left(T_{2}, \rho_{2}\right)^{1}$. Zudem wird das Testgas in Richtung ND-Teil beschleunigt. Die Geschwindigkeit des Testgases ist ca. halb so groß wie die der einfallenden Stoßwelle, siehe Abbildung (2.1). Am Ende des ND-Teils wird die einfallende Stoßwelle reflektiert (reflektierte Stoßwelle) und läuft zurück in Richtung HD-Teil durch das entgegenströmende Testgas. Die Zustandsgrößen des Testgases werden dadurch nochmals deutlich erhöht von $\left(T_{2}, \rho_{2}\right)$ auf $\left(T_{2 R}, \rho_{2 R}\right)$. Im Idealfall ist das Gas hinter der reflektierten Stoßwelle im Ruhezustand. Die weiteren Druckausgleichsvorgänge sind sehr komplex. So kann z. B. der Zustand hinter der reflektierten Stoßwelle auf dreierlei verschiedene Weise gestört werden.

- Bei einem zu kurzen HD-Teil treffen als erstes die am HD-Ende reflektierten Verdünnungswellen, der sogenannte Verdünnungsfächer, zu früh ein.

- Die reflektierte Stoßwelle wird an der Mediengrenze, die Test- und Treibgas voneinander trennt, gebrochen. Entweder als Stoßwelle oder als Verdünngswelle in Richtung des ND-Endes.

In jedem Fall ändern sich durch diese Störungen der Druck und die Temperatur.

\footnotetext{
${ }^{1}$ In der Theorie zur Berechnung von Stoßwellen wird oftmals die Zustandsgröße Dichte $\rho$ des Gases anstatt des Druckes $P$ verwendet.
} 


\subsubsection{Quantitative Theorie des einfachen Stoßwellenrohres}

Der Gaszustand hinter der einfallenden (reflektierten) Stoßwelle kann aus der Stoßwellengeschwindigkeit und dem Ausgangszustand des Gases berechnet werden. Die hydrodynamische Behandlung des Problems führt zu den Rankine-Hugoniot-Gleichungen. Diese Gleichungen werden aus den Erhaltungssätzen des Masse-, Impuls- und Energiestromes unter den idealisierenden Annahmen einer eindimensionalen, stationären Strömung ohne Wärme- und Reibungsverluste an die Stoßrohrwand abgeleitet.

$$
\begin{aligned}
\text { Massenstrom } \Phi_{m} & =\rho_{1} u_{1}=\rho_{2} u_{2} \\
\text { Impulsstrom } \Phi_{i} & =\rho_{1} u_{1}^{2}+p_{1}=\rho_{2} u_{2}^{2}+p_{2} \\
\text { Energiestrom } \Phi_{e} & =u_{1}^{2} / 2+H_{1}=u_{2}^{2} / 2+H_{2}
\end{aligned}
$$

Die Indizes (1) bzw. (2) charakterisieren den Zustand vor dem Stoß (1) bzw. hinter der einfallenden Stoßwelle (2). Dabei bedeuten $\rho$ die Dichte des Gases, $u$ die Geschwindigkeit des Gases relativ zur Stoßfront und $H$ die spezifische Enthalpie pro Masse. Für ein ideales Gas konstanter Wärmekapazität erhält man für den Zustand hinter der einfallenden und reflektierten (Index 2R) Stoßwelle in Abhängigkeit von $f$.

$f$ bezeichnet die Anzahl der Freiheitsgrade des Testgases $(f(\mathrm{Ar})=3)$ und $M$ die MACH-Zahl der einfallenden Stoßwelle bezogen auf das kalte Testgases.

$$
\begin{aligned}
\frac{P_{2}}{P_{1}} & =\frac{(2+f) M^{2}-1}{1+f} \\
\frac{T_{2}}{T_{1}} & =\frac{\left(M^{2}+f\right) \cdot\left[(2+f) M^{2}-1\right]}{[(1+f) M]^{2}} \\
\frac{P_{2 R}}{P_{2}} & =\frac{(3+f) M^{2}-2}{M^{2}+f} \\
\frac{T_{2 R}}{T_{2}} & =\frac{(3+f) M^{2}-2}{M^{2}+f} \cdot \frac{2 M^{2}+f-1}{(2+f) M^{2}-1}
\end{aligned}
$$

Der zum Platzen der Membran notwendige Berstdruck $P_{5}$ berechnet sich aus der MACH-Zahl, den Freiheitsgraden $\left(f_{L}, f_{T}\right)$ und den Schallgeschwindigkeiten $\left(a_{L}, a_{T}\right)$ des Lauf- und Treibgases (Indizes $L, T$ ).

$$
\frac{P_{5}}{P_{2}}=\left[1-\frac{f_{L} \cdot a_{L}}{f_{T} \cdot a_{T}} \cdot \frac{M^{2}-1}{\left(1+f_{L}\right) M}\right]^{-\left(2+f_{T}\right)}
$$

Einige Lehrbuchautoren beschreiben den Zustand hinter Stoßwellen in Abhängigkeit vom Verhältnis der spezifischen Wärmen $\kappa=c_{p} / c_{v}$ anstatt der Anzahl der Freiheitsgrade. Mit $f=2 /(\kappa-1)$ lassen sich die Gleichungen ineinander umformen.

Legt man die Rankine-Hugoniot-Gleichungen zugrunde, läßt sich ein Zeit-Weg-Diagramm der Stoß- und Expansionswellen sowie der Gasströmungen aufstellen. Aus diesem Diagramm können die zu erwartenden Beobachtungszeiten in der Meßebene abgelesen weden. In Abbildung (2.1) ist das Zeit-Weg-Diagramm mit einer in dieser Arbeit typischen Stoßwellengeschwindigkeit von $3 \mathrm{MACH}$ aufgetragen. Die Gaszustände im Stoßrohr wurden entsprechend dem Buch von Oertel [6, Seite 392] numeriert.

(1) Testgas vor dem Stoß 


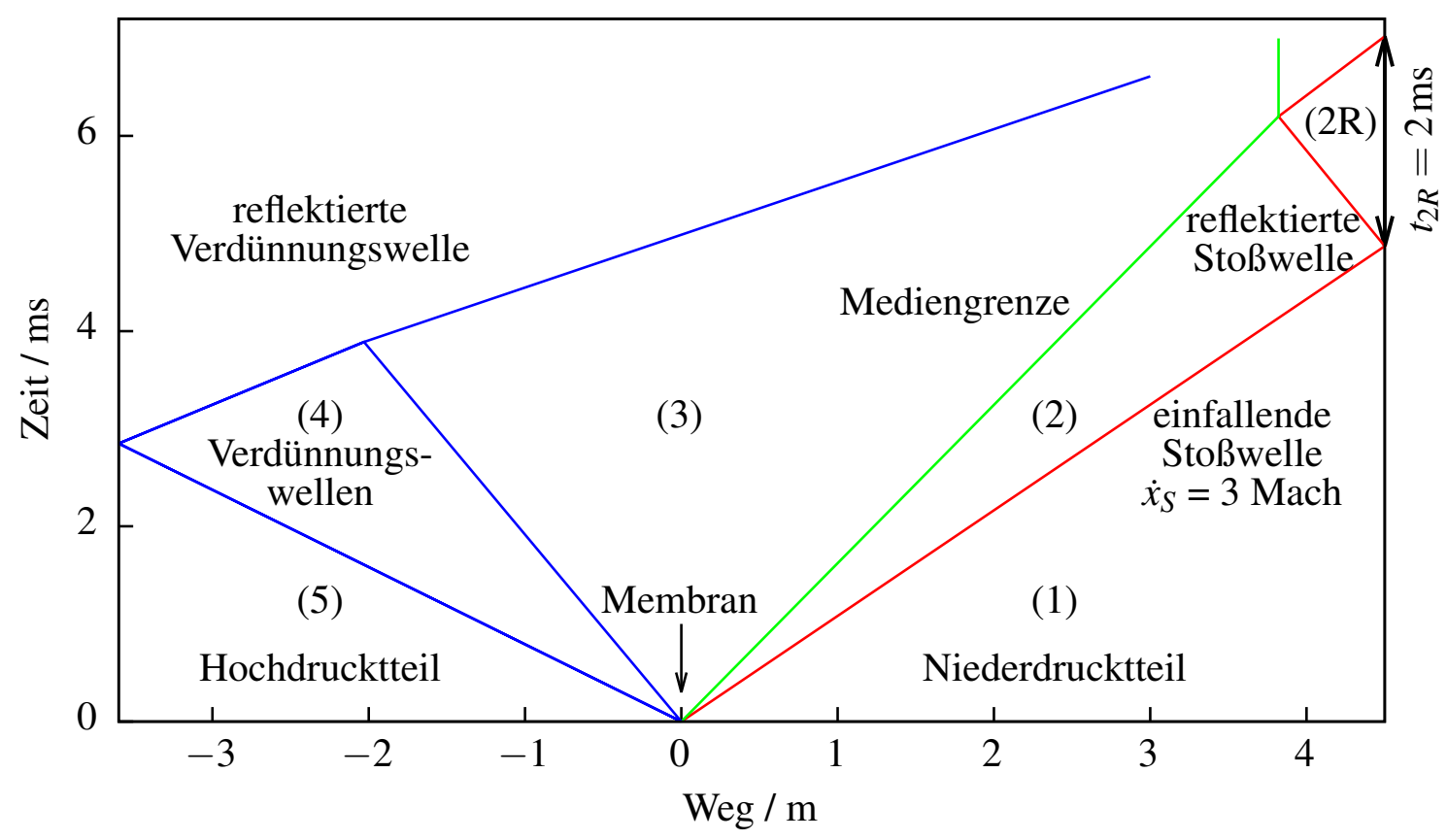

Abbildung 2.1: Ideal berechnetes Zeit-Weg-Diagramm der Stoß- und Verdünnungswellen sowie der Gasströmungen im verwendeten Hochdruckstoßrohr, mit Wasserstoff als Treibgas und Argon als Laufgas $\left(T_{1}=T_{5}=273 \mathrm{~K}\right.$ und $\left.P_{5} / P_{1}=22,6\right)$. Die Geschwindigkeit $\dot{x}_{S}$ der einfallenden Stoßwelle beträgt $3 \mathrm{MACH}$ bezüglich des kalten Testgases. Die fünf verschiedenen Gaszustände im Stoßrohr wurden nach Oertel [6] numeriert.

(2) Testgas hinter der einfallenden Stoßwelle

(2R) Testgas hinter der reflektierten Stoßwelle

(3) Treibgas zwischen dem Ende der Expansionswellen und der Mediengrenze

(4) Treibgas nach dem Durchlaufen der Front der Expansionswellen

(5) Treibgas vor dem Durchlaufen der Expansionswellen

Die Beobachtungszeit $t_{2 R}$ hinter der reflektierten Stoßwelle, an der Stelle $27 \mathrm{~mm}$ vor dem Ende des Niederdruckteils, beträgt im Idealfall 1,9 ms. Mit einer $12 \mathrm{~cm}$ langen Verlängerung beträgt die Beobachtungszeit $t_{2}$ hinter der einfallenden Stoßwelle in derselben Meßebene 0,42 ms Laborzeit. Die Multiplikation der Laborzeit mit dem Dichteverhältnis $\left(\rho_{2} / \rho_{1}\right)$ ergibt die reale Meßzeit. Im Anhang sind sechs Abbildungen (5.1 bis 5.6) dargestellt, aus denen Zeit-Weg-Diagramme für verschiedene Stoßwellengeschwindigkeiten konstruiert werden können. In Abbildung (5.1) sind zusätzlich Meßwerte für das Druckverhältnis $\left(P_{5} / P_{1}\right)$ im Bereich von 2,5 bis 4,0 MACH dargestellt. Durch die Dämpfung der Stoßwellen liegen die Meßwerte etwas über den ideal berechneten Werten. Die oben genannten Gleichungen gelten nur für Testgase mit temperaturunabhängigen Wärmekapazitäten. Mit zunehmendem Anteil mehratomiger Moleküle im Testgasgemisch nimmt die Temperaturabhängigkeit der Wärmekapazitäten zu. In diesem Fall werden die Zustandsgrößen iterativ auf einem Computer berechnet. 


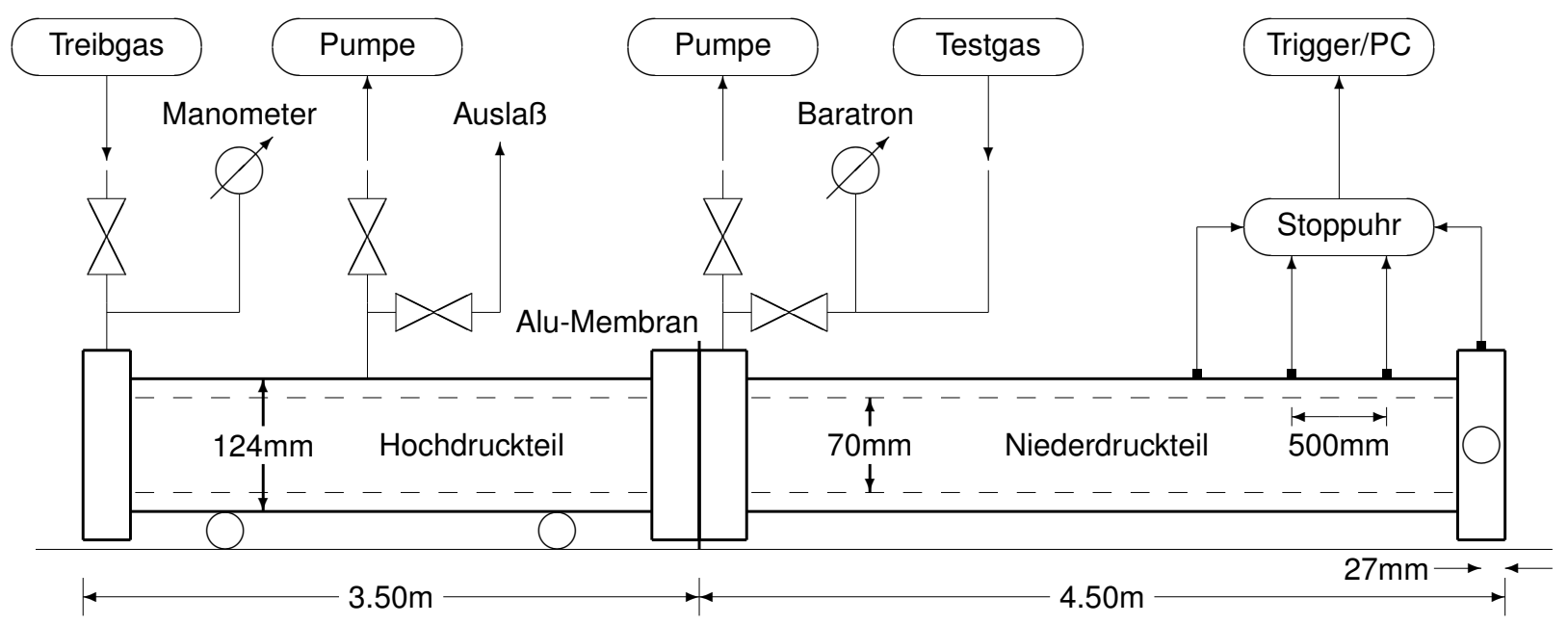

Abbildung 2.2: Skizze des Hochdruckstoßrohres (nicht maßstabsgetreu)

\subsection{Die Stoßwellenapparatur}

Für alle Versuche wurde ein Hochdruckstoßrohr, siehe Abbildung (2.2) verwendet, das in der Dissertation von Henrici [9] und in [10] ausführlich beschrieben wurde. Mit kleinen Modifikationen wurde das Stoßrohr von Wolff [11] neu aufgebaut. Das Stahl-Stoßrohr hatte einen kreisrunden Innendurchmesser von 70,0 mm, eine Wandstärke von $27 \mathrm{~mm}$, einen 3,50 m langen Hochdruckteil und einen 4,50 m langen Niederdruckteil. Zusammengesetzt war das Rohr aus 2 und 0,5 m langen Segmenten. Diese Segmente schlossen beidseitig mit $20 \times 20 \times 6 \mathrm{~cm}^{3}$ großen Flanschen ab, die zur Verbindung zum folgenden Rohrsegment und zur Aufnahme von Detektoren, Fenstern und Ventilen dienten. ND- und HD-Teil wurden von je einer schweren Endplatte abgeschlossen.

Vor jedem Experiment wurden beide Seiten des Stoßrohres auf einen von Druck von ca. 1 Pa evakuiert. Dazu wurden Öl-Drehschieberpumpen vom Typ Leybold D12 und Alcatel W26 eingesetzt. Die Leckrate war stets kleiner als $10^{-3} \mathrm{~Pa} \mathrm{~m}^{3} / \mathrm{s}$.

Die Stoßwellengeschwindigkeit wurde mit vier Platin-Widerstandsstreifen gemessen, die im Abstand von $500 \mathrm{~mm}$ in die Flansche eingeschraubt waren. Der letzte Meßpunkt lag in der Meßebene $27 \mathrm{~mm}$ von der Endplatte entfernt. Zur Aufnahme von hochaufgelösten Druck-Zeit-Profilen konnten die Platin-Widerstandsstreifen gegen beschleunigungskompensierte piezoelektrische Druckaufnehmer mit hoher Eigenfrequenz von Firma Kistler (Typ 603B) ersetzt werden. In Höhe des letzten Platin-Widerstandsstreifen waren zwei um $180^{\circ}$ versetzte Fensterbohrungen angebracht. In diese wurden je nach Aufgabenstellung verschiedene Typen von Duran-Fenstern eingesetzt. Es wurden große Fenster $(\varnothing=14 \mathrm{~mm})$, kleine Fenster $(\varnothing=6 \mathrm{~mm})$ und Doppelfenster verwendet. Das Doppelfenster besteht aus zwei im Winkel von $6^{\circ}$ zueinander stehenden kleinen Fenstern. Es wurde eingesetzt, um zwei Laserstrahlen gekreuzt durch das Stoßrohr zu führen. Aufgrund der höheren Betriebssicherheit wurde - wann immer möglich — mit kleinen Fenster gearbeitet. Zur Verlängerung der Beobachtungszeit hinter der einfallenden Stoßwelle, konnte das Rohr am ND-Teilende um zwei jeweils $60 \mathrm{~mm}$ lange Segmente verlängert werden. Die von Wolff [11] verwendeten Ein- und Auslaßventile von Firma Hofer (Typ PN400) wurden im Laufe dieser Arbeit gegen Ventile von Firma Nupro (Typ 12PT6 und DLS8) ausgetauscht, wodurch die Leckrate verringert wurde. 


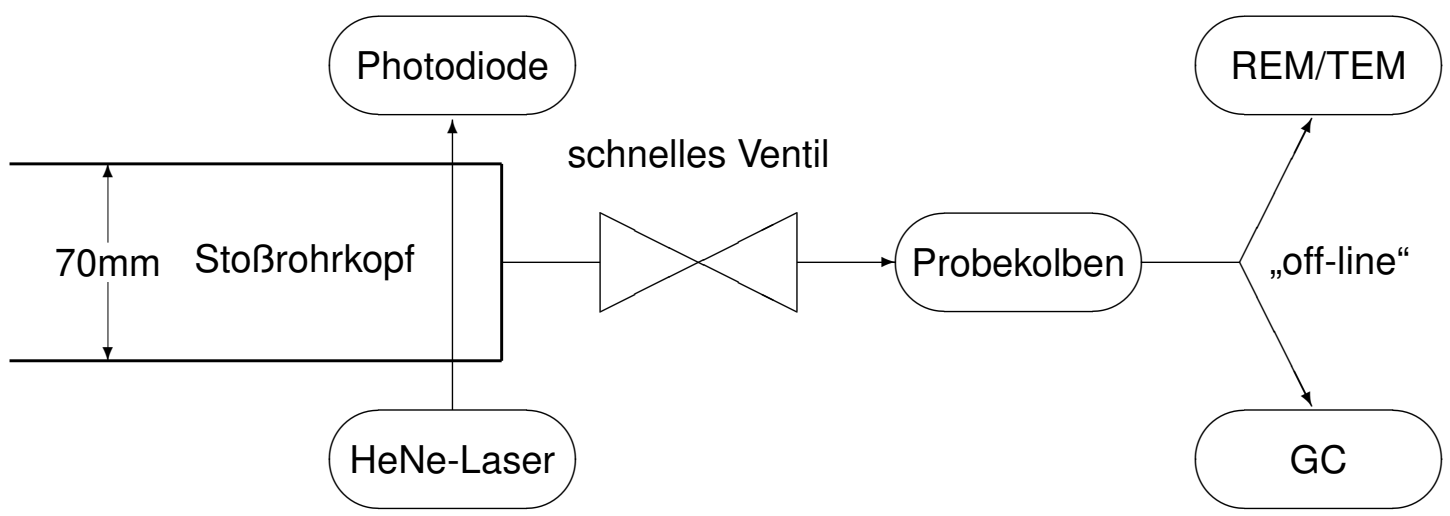

Abbildung 2.3: Schematischer Aufbau, der Optik und des Gasanalysesystems.

\subsubsection{Membran}

Zwischen ND- und HD-Teil wurden Aluminiummembranen unterschiedlicher Dicke eingespannt: $0,05 \leq d_{\text {Membran }} / \mathrm{mm} \leq 2$. Für Membranen dicker als $0,1 \mathrm{~mm}$ wurde stets weiches Aluminium verwendet. Hartes Material splittert in scharfkantige Bruchstücke, die Fenster und Detektoren beschädigen können. Der Berstdruck $P_{5}$ ist für das verwendete Stoßrohr annähernd direkt proportional zur Membrandicke:

$$
P_{5} \approx 3 \mathrm{MPa} \cdot d_{\text {Membran }} / \mathrm{mm}
$$

Entscheidend für die Reproduzierbarkeit von Experimenten ist, daß die Streuung des Berstdruckes von Membranen möglichst klein ist. Von wenigen Ausnahmen abgesehen, waren die Experimente mit einer Abweichung des Druckes $\Delta P_{2 R}<2 \%$ reproduzierbar.

\subsection{Optischer Aufbau}

Die Rußbildung wurde durch die Absorption von Laserlicht beobachtet. In Abbildung (2.3) ist der Aufbau der Optik schematisch dargestellt. Als Lichtquellen dienten ein Helium-Neon Laser von Firma Uniphase (Typ 1125P, $\lambda=632,8 \mathrm{~nm}, P=10 \mathrm{~mW}$ ), der im $c w$-Betrieb lief und eine Infrarot-Laser-Diode von Firma EG\&G (Typ C86125E, $\lambda=1064 \mathrm{~nm}, P=50 \mathrm{~mW}$ ), die elektronisch bis zu $400 \mathrm{kHz}$ mit Rechtecksignalen gepulst wurde. Die Laserstrahlen wurden im Winkel von $6^{\circ}$ zueinander in der Meßebene senkrecht durch das Stoßrohr geführt. Das Licht des HeNe-Lasers wurde mit einer Photodiode der Firma Hamamatsu (Typ SS1133-03) detektiert. Zuvor wurde das Licht aus Störquellen (z. B. Emission des Rußes) mit einem $1 \mathrm{~mm}$ Spalt und einem schmalbandigen Interferenzfilter (FWHM $=8 \mathrm{~nm}$, Transmission $50 \%$ ) ausgeblendet. Das Laser-Dioden-Licht wurde mit einem Universalkollimator von Firma Laser Components (Typ SK9650) in die Mitte des Stoßrohres fokussiert und mit einer Photodiode von Firma EG\&G (Typ YAG200) detektiert. Ein Interferenzfilter $(F W H M=25 \mathrm{~nm})$ und ein $1 \mathrm{~mm}$ Spalt blendeten das Störlicht aus.

\subsubsection{Signalerfassung und EDV}

Alle elektronischen Signale der Platin-Widerstandsstreifen, Kistler-Drucksonden und Photodioden wurden vorverstärkt und über zwei parallel geschaltete Zwei-Kanal-AnalogDigital-Wandler-Karten von Firma Imtec (Typ T3240) in einem IBM/PC aufgezeichnet. 
Die AD-Karten arbeiteten mit einer Abtastfrequenz von bis zu $20 \mathrm{MHz}$, einer Speichertiefe von $16 \mathrm{k}$ byte und einer 8 bit-Auflösung. Die Daten wurden mit einem TURBO-PASCAL Programm in ASCII-Format konvertiert und mit Tabellenkalkulationsprogrammen aufgearbeitet. Der Beginn der Signalaufzeichnug wurde von dem Platin-Widerstandsstreifen bzw. der Kistler-Drucksonde in der Meßebene getriggert.

\subsection{Absorption von Licht durch Rußpartikel}

Die Absorption des Laserlichtes durch Rußpartikel wird mit Hilfe des Lambert-Beerschen Gesetzes beschrieben. Mit dem natürlichen Absorptionskoeffizienten $\alpha$ in der Einheit $\mathrm{m}^{2} / \mathrm{mol}$ läßt sich dann die Kohlenstoffkonzentration $[C]_{\mathrm{Ru} \beta}$, die in Form von Ruß vorliegt, in der Einheit $\mathrm{mol} / \mathrm{m}^{3}$ berechnen, siehe Gleichung (2.10).

$$
[C]_{\mathrm{Ru} \beta}=\frac{\ln \left(I_{0} / I_{t}\right)}{L \cdot \alpha} \quad\left[\frac{\mathrm{mol}}{\mathrm{m}^{3}}\right]
$$

Die optische Weglänge $L$ ist gleich dem Durchmesser des Stoßrohres (70,0 mm). Der natürliche Absorptionskoeffizient $\alpha$ wird mit Hilfe der Mieschen Theorie über die Wechselwirkung von Licht mit kleinen Kugeln berechnet [12, 13]. Unter der Annahme, daß die

- Rußpartikel während des Wachstums im Stoßrohr homogen verteilt sind,

- Partikel annähernd Kugelgestalt haben,

- Durchmesser dieser Partikel viel kleiner sind als die Wellenlänge des Laserlichtes $\left(d_{\text {Rußpartikel }} / \lambda_{\text {Laserlicht }}\right)<0,1$,

- Partikel soweit voneinander entfernt sind, daß sich die induzierten Dipole nicht gegenseitig beeinflussen,

gilt die Rayleigh-Näherung. Die Mieschen Gleichungen können dann in verkürzter Form verwendet werden. Für den natürlichen Absorptionskoeffizienten $\alpha$ gilt:

$$
\alpha=\frac{6 \pi \cdot M_{C}}{\lambda \cdot \rho_{\mathrm{Ru} \beta}} \cdot \mathfrak{I}\left(\frac{m^{2}-1}{m^{2}+2}\right) \quad\left[\frac{\mathrm{m}^{2}}{\mathrm{~mol}}\right]
$$

Diese Funktion ist an der Stelle $(k=\sqrt{2}, n \rightarrow 0)$ unstetig und strebt gegen unendlich. In obiger Gleichung bedeuteten: $M_{C}$ die Molmasse des Kohlenstoffs; $\rho_{\mathrm{Ru}}$ die spezifische Dichte von Ruß $\left(\rho_{\mathrm{Ruß}}=1860 \mathrm{~kg} / \mathrm{m}^{3}[1]\right), \lambda$ die Wellenlänge des Laserlichts und $m=(n-i \cdot k)$ der komplexe Brechungsindex des Rußes, mit dem Realteil $n$ und dem Imaginärteil $k$. Unter den oben genannten Annahmen ist der gestreute Anteil des Lichtes klein gegenüber dem absorbierten Anteil. Der natürliche Absorptionskoeffizient des Rußes für Licht der Wellenlänge $(\lambda=632,8 \mathrm{~nm})$ ist in Abbildung (2.4), in den für die Rußbildung interessierenden Bereich, als Funktion des komplexen Brechungsindex dargestellt. Zusätzlich sind experimentelle Meßwerte für Ruß von verschiedenen Autoren aufgenommen. Der Brechungsindex $(n=1,57, k=0,56)$ ist ein häufig verwendeter Wert, und als Literaturquelle wird „Dalzell,Sarofim“ angegeben [14]. Tatsächlich wurde von Dalzell und Sarofim in der Veröffentlichung der Brechungsindex für Acetylenruß bei $\lambda=650 \mathrm{~nm}$ mit $(n=1,57, k=0,44)$ angegeben. 


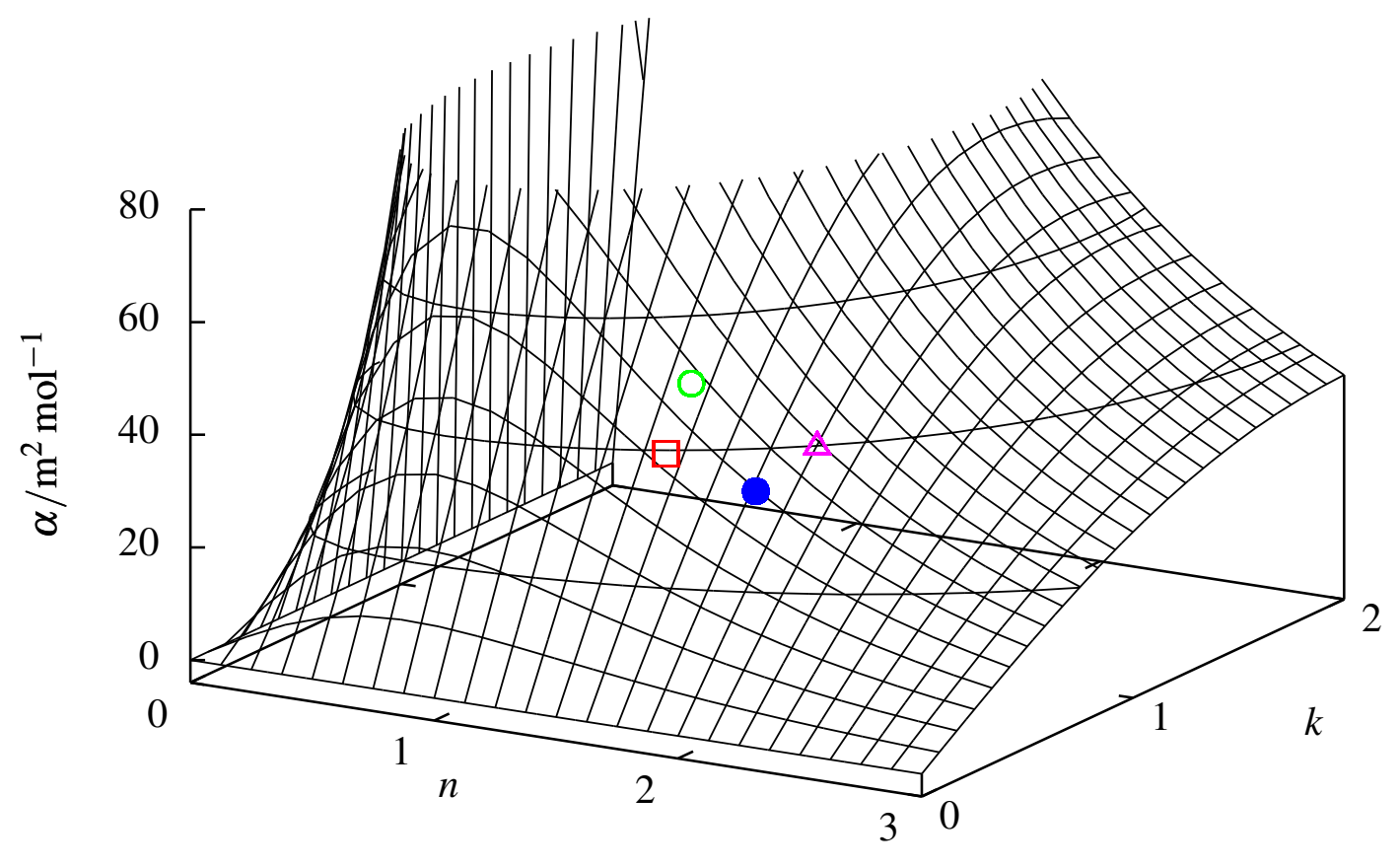

Abbildung 2.4: Natürlicher Absorptionskoeffizient $\alpha$ des Rußes bei $2000 \mathrm{~K}$ für Licht der Wellenlänge $(\lambda=632,8 \mathrm{~nm})$ in Abhängigkeit des komplexen Brechungsindex $(m=n-i$. $k$ ) und Meßwerte von verschiedenen Autoren. $\square$ : Dalzell,Sarofim, ○: „Dalzell,Sarofim“, $\bullet$ : Lee und Tien $n=1.9 k=0.5 \alpha=34 \mathrm{~m}^{2} / \mathrm{mol}$ und $\triangle$ : Senftleben.

In dieser Arbeit wird der wellenlängen- und temperaturabhängige Brechungsindex von Lee und Tien [15] angewendet, der gut mit den berechneten Kohlenstoff-Bilanzen aus den gemessenen Gasanalysen in Abschnitt (3.6) korreliert.

\subsection{Probenentnahme und Gasanalyse}

Die Auswertung der Absorptionsprofile mit dem Brechungsindex von Lee und Tien, siehe Kapitel (3.4) zeigt, daß die Rußausbeuten in einer Kohlenwasserstoffpyrolyse stets kleiner als $100 \%$ sind. Daher ist es von großem Interesse, die anderen Reaktionsprodukte quantitativ zu analysieren. Zu diesem Zweck wurde ein Probenentnahmesystem entwickelt, mit dem es möglich wurde, unmittelbar aus dem Gas hinter der reflektierten Stoßwelle eine repräsentative Probe zu entnehmen, die ca. 1 bis $5 \%$ des gesamten Testgases enthält.

\subsubsection{Ventil}

Aus dem Testgasgemisch soll eine Gasprobe entnommen und analysiert werden. Dazu wurde unmittelbar nach dem Experiment hinter der reflektierten Stoßwelle, d. h. etwa zeitgleich mit dem Eintreffen des reflektierten Verdünnungsfächers ein Ventil am Stoßrohrkopf geöffnet und ein kleiner Teil des Testgases in einen evakuierten Metallzylinder expandiert. Mit der schnellen Gasexpansion sinkt auch die Temperatur des Testgases. Somit wurden die Reaktionen im Testgas „eingefroren“. Um eine unkontrollierte Verdünnung der Probe mit dem Treibgas (Wasserstoff) auszuschließen, wurde spätestens vor dem Erreichen des Treibgases, aus der nunmehr verwirbelten Mediengrenze zwischen 


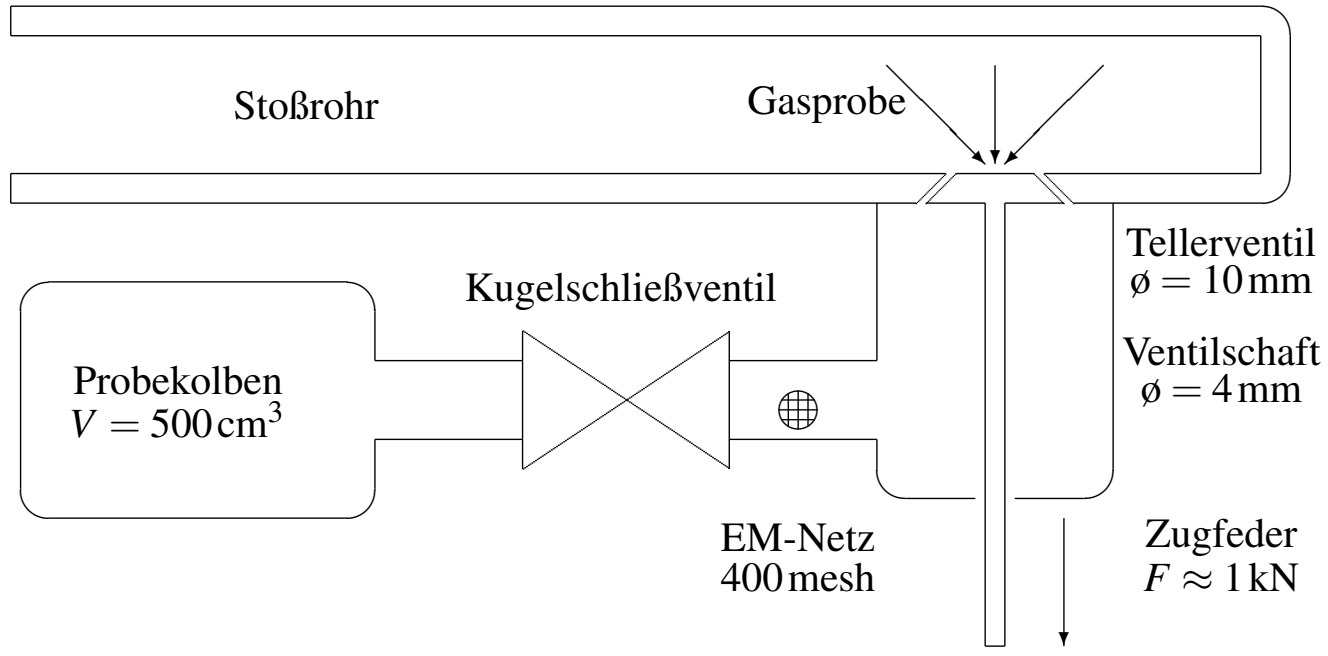

Abbildung 2.5: Schematischer Aufbau des Probenentnahmesystem am Hochdruckstoßrohr

Testgas und Treibgas, das Rohr wieder verschlossen. Da das „Aufwirbeln“ der Kontaktfläche innerhalb einiger Millisekunden geschah, mußte die Probenentnahme mindestens ebenso schnell sein. An das Öffnungs- und Schließsystem (Ventil) wurden also folgende Anforderungen gestellt:

- Vor dem Experiment dichtete das Ventil zwischen Stoßrohr und evakuierten Probezylinder ab. Die Druckdifferenz betrug ca. 0,1 MPa.

- Das Ventil paßte sich dem kreisrunden Querschnitt des Stoßrohres an. Größere Unebenheiten $( \pm 0,5 \mathrm{~mm})$ der Stoßrohrwand hätten störend sowohl auf die einfallende als auch die reflektierte Stoßwelle gewirkt.

- Während des Experiments hielt das Ventil der schnellen Druckzunahne auf zu $10 \mathrm{MPa}$ stand.

- Ca. 1 ms nach dem Eintreffen des Verdünnungsfächers öffnete das Ventil, gab einen hinreichend großen Querschnitt frei und schloß nach weiteren ca. $10 \mathrm{~ms}$. Während der Öffnungszeit sollten einige Prozent des Testgases in den Probekolben strömen.

- Bis zum Druckablassen aus dem Stoßrohr herrschte über dem Ventil eine Druckdifferenz von ca. $2 \mathrm{MPa}$

- Die Oberfläche des Probenentnahmesystems wirkte weder katalytisch auf das zunächst noch heiße Testgas noch wurden die zu analysierenden Gase adsorbiert.

Das Ventil wurde in den Institutswerkstätten angefertigt ${ }^{2}$. Die wesentlichen Konstrukionsmerkmale des Probenentnahmesystems sind in Abbildung (2.5) dargestellt. In den Stoßrohrflansch wurde ebenwandig zur Stoßrohrinnenwand ein O-Ring gedichtetes Tellerventil eingepaßt. Von dort führte eine $10 \mathrm{~mm}$ dicke und $0,5 \mathrm{~m}$ lange Rohrleitung über ein Kugelventil in einen $500 \mathrm{~cm}^{3}$ großen Probezylinder. Über vorgespannte und zunächst arretierte Zugfedern konnte das Tellerventil geöffnet und das Kugelventil geschlossen

\footnotetext{
${ }^{2}$ An dieser Stelle sei nochmals den Mitarbeiten der Werkstätten gedankt, die mich beim Auf- und Umbau des Sytems stets schnell und hilfreich unterstützten.
} 


\begin{tabular}{|c|c|c|}
\hline GC-Parameter & GC 1 & GC 2 \\
\hline nachweisbare Gase & $\mathrm{C}_{n} \mathrm{H}_{m}$ mit $n \leq 3 m \leq 8$ & $\mathrm{He} \mathrm{H}_{2} \mathrm{O}_{2} \mathrm{~N}_{2} \mathrm{CO} \mathrm{CH}_{4}$ \\
\hline Probenschleife & ca. $100^{\circ} \mathrm{C}$ & ca. $100^{\circ} \mathrm{C}$ \\
\hline Injektor & $170^{\circ} \mathrm{C}$ & $170^{\circ} \mathrm{C}$ \\
\hline Säule & Carbosieve G, $2 \mathrm{~m}$ & 5 Å Molekularsieb, 2 m \\
\hline Temperatur & $130^{\circ} \mathrm{C}$ & $\begin{array}{l}110^{\circ} \mathrm{C} \text { und }-20^{\circ} \mathrm{C} \\
\text { zur } \mathrm{He} / \mathrm{H}_{2} \text {-Trennung }\end{array}$ \\
\hline Detektor & FID $\mathrm{H}_{2} /$ Luft KW-frei & WLD $100 \mathrm{~mA}$ \\
\hline Temperatur & $170^{\circ} \mathrm{C}$ & $170^{\circ} \mathrm{C}$ \\
\hline Trägergas & Helium & Argon \\
\hline Volumensstrom & $300 \mathrm{~mm}^{3} / \mathrm{s}$ & $110 \mathrm{~mm}^{3} / \mathrm{s}$ \\
\hline
\end{tabular}

Tabelle 2.1: Betriebsparameter der Gaschromatographen

werden. Diese Arretierungen wurden unabhängig voneinander vom elektronisch verstärkten und passend zeitverzögerten Triggersignal des letzten Platin-Widerstandsstreifens gelöst. Rußpartikelproben für die elektronenmikroskopische Analyse wurden unmittelbar hinter dem Schließventil auf längs zur Strömung angebrachten Netzen und Aluminiumfolien entnommen. Mit diesem Ventilmechanismus wurden einige Prozent Testgas innerhalb von $10 \mathrm{~ms}$ abgesaugt, mit einer Gasgeschwindigkeit von einigen hundert Metern pro Sekunde durch die $10 \mathrm{~mm}$ dicke Rohrleitung geführt und in dem $500 \mathrm{~cm}^{3}$ großen Probezylinder aufgefangen. Auf die Vorderseite des Tellerventils wurde ein runder Spiegel $(\varnothing=6 \mathrm{~mm})$ fixiert. Ein durch das gegenüberliegende Doppelfenster eingekoppelter Laserstrahl wurde an dem Spiegel reflektiert und im Winkel von $6^{\circ}$ zum einfallenden Laserstrahl durch dasselbe Fenster wieder ausgekoppelt, und die optische Weglänge wurde verdoppelt. Damit war es möglich, den Rußbildungsprozeß auch bei ProbenentnahmeExperimenten absorptionsspektroskopisch zu verfolgen.

\subsubsection{Gaschromatographische Analyse}

Für die Gasanalyse wurden zwei Gaschromatographen von Packard-Becker (Modell 419) verwendet von denen der erste (GC1) mit 60/80 mesh Carbosieve G-Säulen $\left(5 \mathrm{ft} \times 1,8^{\prime \prime}\right)$ und Flammenionisationsdetektoren (FID), der zweite (GC2) mit $5 \AA$ Molekularsieb-Säulen und Wärmeleitfähigkeitsdetektoren (WLD) ausgestattet waren. Auf dem GC1 konnten $C_{1}$ bis $C_{3}$ Kohlenwasserstoffe, auf dem GC2 die Permanentgase $\mathrm{He}, \mathrm{H}_{2}, \mathrm{O}_{2}, \mathrm{~N}_{2}, \mathrm{CO}$ und $\mathrm{CH}_{4}$ nachgewiesen werden. Die Detektorensignale wurden von einem Zweikanalintegrator von Spektra-Physics (Modell SP4270) verarbeitet, und die Flächen unter den Signalpeaks in willkürlichen Einheiten mit Angabe der Retentionszeit auf dem Schreiber ausgegeben. Injiziert wurden die Testgasproben über $0,2 \mathrm{ml}$ große thermostatierte Probeschleifen und bei optimierten Betriebsparametern, siehe Tabelle (2.1) in Basispeaks getrennt. Die Retentionszeiten und Nachweisgrenzen der analysierten Gase sind in Tabelle (2.2) angegeben. Jede entnommene Gasprobe wurde dreimal analysiert.

Zur Eichung der Gaschromatographen wurden Eichgemische in 10 L-Druckgasflaschen präpariert, mit denen vor und nach den Messungen die Empfindlichkeit der Detektoren bestimmt wurde. Die Gemischzusammensetzung des Eichgases orientierte sich an der zu erwartenden Testgaszusammensetzung. Während der ca. einhundert Eichungen einer Meßserie, die innerhalb von einigen Wochen aufgenommen wurde, blieben die Detektorempfindlichkeiten hinreichend konstant. Die Partialdrücke wurden über mehr als zwei 


\begin{tabular}{llcc}
\hline $\mathrm{GC}$ & $\mathrm{Gas}$ & Retentionszeit / min & Nachweisgrenze / ppm \\
\hline 1 mit FID & $\mathrm{CH}_{4}$ & 2,2 & 1 \\
& $\mathrm{C}_{2} \mathrm{H}_{2}$ & 12,1 & 100 \\
& $\mathrm{C}_{2} \mathrm{H}_{4}$ & 17,3 & 1 \\
& $\mathrm{C}_{2} \mathrm{H}_{6}$ & 25,6 & 1 \\
\hline 2 mit WLD & $\mathrm{He}$ & - & 10 \\
& $\mathrm{H}_{2}$ & 4,3 & 10 \\
& $\mathrm{O}_{2}$ & 5,3 & 10 \\
& $\mathrm{~N}_{2}$ & 6,2 & 10 \\
& $\mathrm{CH}_{4}$ & 13,0 & 100 \\
& $\mathrm{CO}$ & 16,0 & 100 \\
\hline
\end{tabular}

Tabelle 2.2: Retentionszeiten und Nachweisgrenzen quantitativ analysierter Gase

Größenordnungen variiert. Erwartungsgemäß konnten die Auftragungen Partialdruck des Eichgases gegen die Peakfläche mit Geraden angepaßt werden. Ihr Ordinatenabschnitt war stets gleich null. Eine überraschende Ausnahme war Ethin. Zu kleinen Ethin-Konzentrationen verringerte sich die Detektorempfindlichkeit stetig. Deswegen wurde die Acetyleneichkurve in mehrere Abschnitte unterteilt, die jeweils mit einer Geraden approximiert wurde. Mit Hilfe von Grenzgeraden wurde für die Eichgeraden ein relativer Fehler von $\pm 7 \%$ ermittelt.

\subsubsection{Elektronenmikroskopie}

Es wurden Rußproben mit vier verschiedenen Methoden gesammelt:

- Unmittelbar hinter dem Schließventil wurde ein kleines Aluminiumblech längs der Gasströmungs so fixiert, daß die Gasströmung möglichst wenig beeinflußt wurde.

- Unmittelbar dahinter wurden Kupfer-Netzchen mit Kohlenstofffilmauflage ( $\varnothing=$ $3,05 \mathrm{~mm}, 400 \mathrm{mesh}$ ) in einem strömungsgünstig angebrachten Adapter fixiert.

- Auf die Endplatte des Niederdruckteils wurden Aluminiumfolien gespannt.

- Nach Experimenten mit hohen Kohlenstoffdichten blieb Ruß im Stoßrohr liegen. Dieser Ruß wurde eingesammelt und in Schnappdeckelgläschen aufbewahrt.

Die Strukturuntersuchungen der Rußproben wurden im Institut für Metallphysik der Universität Göttingen elektronenmikroskopisch vorgenommen. Dazu standen sowohl ein Rasterelektronenmikroskop (REM) als auch ein Transmissionselektronenmikroskop (TEM) zur Verfügung ${ }^{3}$. Das REM ist ein Industriegerät von Philipps (Modell SEM 515) mit einer maximalen Beschleunigungsspannug von $30 \mathrm{kV}$ und einer maximalen Vergrößerung von $1,6 \cdot 10^{5}$. Jeweils sechs Proben wurden auf ein Zoll große Aluminium-Träger präpariert und im Sputter möglichst dünn (ca. $15 \mathrm{~nm}$ ) mit Gold beschichtet. Jede Probe wurde auf Homogenität untersucht, indem die Probe von einem Rand zum gegenüberliegenden verschoben, und an verschiedenen Stellen mit einer $35 \mathrm{~mm}$ Kamera fotografiert wurde.

\footnotetext{
${ }^{3}$ An dieser Stelle bedanke ich mich bei Herrn Hahn und Herrn Dr. Wilbrandt, die mir bei den elektronenmikroskopischen Aufnahmen geholfen haben.
} 
Die Netze wurden einzeln auf einem Probenträger fixiert und in das Philipps-TEM (Modell EM 400T) eingeführt. Das Mikroskop arbeitete mit der maximalen Beschleunigungsspannug von $120 \mathrm{kV}$ und einer Vergrößerung von bis zu 4,8 $10^{5}$. Die Aufnahmen wurden auf $8 \mathrm{~cm} \times 10 \mathrm{~cm}$ großen Fotoplatten dokumentiert. Aus den zehn- bis zwanzigfach vergrößerten Fotoaufnahmen konnte die Größe der Rußpartikel auf $\pm 20 \%$ bestimmt werden. Die Größe des Fehlers ergibt sich aus den unscharfen Randbereichen der Rußpartikel.

\subsubsection{Gas-Adsorption}

Die Bestimmung der spezifischen Oberfläche von Rußproben wurde im All Russian Scientific Research Institut of Natural Gases and Gas Turbines, „,VNIIGAS“ von Prof. Tesners Arbeitsgruppe in Moskau ausgeführt ${ }^{4}$. Diese Methode wird standardmäßig zur Charakterisierung von Industrierußen angewendet. Es wurde Stickstoff bei der Temperatur von flüssigem Stickstoff auf eine ca. $100 \mathrm{mg}$ große Rußprobe adsorbiert. Aus der adsorbierten Stickstoffmenge und der Querschnittsfläche eines Stickstoffmoleküles ließ sich die spezifische Oberfläche der Probe berechnen. Die Meßgenauigkeit betrug $\pm 5 \%$.

\subsubsection{HPLC}

Zur Analyse der Testgasprobe auf höhere polycyclische Aromaten (PAH) wurde der Probezylinder mit $10 \mathrm{ml}$ Dichlormethan $\left(\mathrm{CH}_{2} \mathrm{Cl}_{2}\right)$ ausgespült und mit der HPLC-Methode untersucht ${ }^{5}$. Eine detaillierte Beschreibung der HPLC-Apparatur und des Analyseverfahrens findet sich in [16]. Die Nachweisgrenze für einzelne polycyclische Aromaten lag bei jeweils ca. $1 \cdot 10^{-7} \mathrm{~g}$. Benzol konnte mit dieser Apparatur nicht bestimmt werden.

\subsubsection{Elementaranalyse}

Im Institut für Anorganische Chemie der Universität Göttingen wurden Elementaranalysen auf den Kohlenstoff- und Wasserstoffgehalt von Rußproben ausgeführt. Die Rußproben wurden nach Experimenten mit hohen Kohlenstoffdichten von der Stoßrohrwand gesammelt und in Schnappdeckelgläsern aufbewahrt. Experiment und Analyse wurden jeweils innerhalb von einer Woche ausgeführt.

\subsection{Chemikalien und Gase}

Die Reinheiten und Lieferanten der verwendeten Gase und Chemikalien sind in Tabelle (2.3) aufgeführt. Die Gase und Chemikalien wurden ohne weitere Reinigung eingesetzt. Phenylacetylen wurde unmittelbar vor Gebrauch über eine Vigreux-Kolonne bei normalen Druck und einer Temperatur von $T=142{ }^{\circ} \mathrm{C}$ destilliert.

Mit einem in früheren Arbeiten [17] beschriebenen Hochdruckgasmischsystem wurden Testgasmischungen und GC-Eichmischungen nach der Partialdruckmethode in vorher ausgeheizte und evakuierte 10 und 50 L-Stahldruckflaschen präpariert. Vor dem Verdampfen von Flüssigkeiten in die Stahlflaschen wurden zunächst leicht flüchtige Verun-

\footnotetext{
${ }^{4}$ An dieser Stelle bedanke ich mich bei Prof. Tesner und seinen Mitarbeitern, die die Messungen in ihrem Labor zügig ausführten.

${ }^{5}$ An dieser Stelle bedanke ich mich bei Frau Dr. Hanisch, die die HPLC-Analysen ausführte.
} 


\begin{tabular}{lll}
\hline Substanz & Lieferant & Reinheit / \% \\
\hline Helium & Messer-Griesheim & 99,996 \\
Wasserstoff & Messer-Griesheim & 99,9 \\
Argon & Messer-Griesheim & 99,998 \\
Stickstoff & Messer-Griesheim & 99,999 \\
Methan & Linde & 99,5 \\
Acetylen & Messer-Griesheim & analysenrein \\
Ethylen & Linde & 99,8 \\
Ethan & Messer-Griesheim & 99,95 \\
\hline Benzol & Merck & 99,7 \\
n-Hexan & Merck & 99,0 \\
Phenylacetylen & Aldrich & 98 \\
Eisenpentacarbonyl & Fluka & 97 \\
Dichlormethan & Aldrich & 99,9 \\
Aceton & - & technisch \\
\hline
\end{tabular}

Tabelle 2.3: Reinheiten und Lieferanten der verwendeten Gase und Chemikalien.

reinigungen durch scharfes Absaugen entfernt. Schwer flüchtige Verunreinigungen reicherten sich im Sumpf des Vorratskolbens an. Die Flaschen wurden bis zu $10 \mathrm{MPa}$ mit Argon aufgefüllt. Vor Verwendung der Gasmischungen wurden sie entweder zwei Tage zur Durchmischung stehengelassen, oder für einige Stunden mit Hilfe thermisch erzeugter Konvektion gemischt. Eine spezielle Stahlflasche mit interner Mischvorrichtung wurde benutzt, um innerhalb weniger Minuten ein Testgas herzustellen.

Die Molenbrüche der Benzolmischungen wurden gaschromatographisch überprüft. Es wurde ein mit Probenschleife, Volaspher-A2-Säulen (mit 3 \% OV17) und Flammenionisationsdetektoren ausgestatteter Packard-Becker-Gaschromatograph (Modell 419) verwendet. Zur Eichung des Gaschromatographen wurden Benzol/Argon-Mischungen in einem temperierten, doppelten Benzolsättiger hergestellt. Zur Verdünnung der Benzol/ArgonMischung wurde ein variabler Argonfluß zugemischt. Somit konnte der Benzolanteil des Eichgemisches zwischen 100 ppm und $5 \%$ variiert werden. Es wurde festgestellt, daß die Adsorption von Benzol auf die Wand der Stahlflasche vernachlässigbar klein ist.

\subsection{Versuchsausführung}

Vor jeder Messung wurde die Laser-Optik überprüft und gegebenfalls nachjustiert, und bei Messungen mit dem Probenentnahmesystem das Ventil „scharf“ gestellt. Anschließend wurde in den Niederdruckteil des Stoßrohrs Testgas mit dem Druck $P_{1}$ (z. B. 1 bar) eingelassen. Bis zum Bersten der Membran wurde über ein Magnetventil Wasserstoff in den Hochdruckteil eingeströmt. Nach dem Experiment wurden die Daten auf dem Computer gesichert und das Stoßrohr mit Aceton und Zellstoff gründlich gereinigt. Die GC-Analysen wurden unmittelbar nach dem Experiment ausgeführt. Die Rußproben wurden über mehrere Versuchstage gesammelt und chargenweise im Elektronenmikroskop untersucht. An Versuchstagen konnten die Experimente im ca. einstündigen Abstand ausgeführt werden. 


\section{Kapitel 3}

\section{Darstellung der Meßergebnisse}

In diesem Kapitel werden die Ergebnisse von Untersuchungen des Rußbildungsprozesses in der Pyrolyse von aromatischen und aliphatischen Kohlenwasserstoffen hinter Stoßwellen dargestellt. Die Mehrzahl der Experimente wurde hinter reflektierten Stoßwellen ausgeführt. Es wurde der Einfluß der Temperatur, der Kohlenstoffkonzentration, des Druckes sowie des Kohlenwasserstoffes untersucht. Die Absorptionsmeßergebnisse beziehen sich auf den Helium-Neon-Laser mit der Wellenlänge $(\lambda=632,8 \mathrm{~nm})$. Wenn sich diese Meßergebnisse von denen der Laserdiode $(\lambda=1064 \mathrm{~nm})$ unterscheiden, wird darauf gesondert eingegangen.

\subsection{Absorption- und Rußausbeute-Zeitprofil}

Anhand eines charakteristischen Intensitäts-Zeitprofils des detektierten HeNe-Laserlichtes werden die wesentlichen Eigenschaften der Rußbildung dargestellt.

Abbildung (3.1) zeigt das auf $I_{0}$ normierte Intensitäts-Zeitprofil sowie das zugehörige Druck-Zeitprofil während der Rußbildung in einer Benzolpyrolyse bei einer Temperatur von $1890 \mathrm{~K}$, einem Druck von 5,5 MPa und einer Kohlenstoffdichte von $[C]$ $=0,84 \mathrm{~mol} / \mathrm{m}^{3}$. Deutlich erkennbar sind die im Abstand von etwa $80 \mu$ s auftretenden Schlierensignale im Intensitätszeitprofil und die Drucksprünge im Druckzeitprofil, die von der einfallenden und reflektierten Stoßwelle verursacht wurden. Hinter der reflektierten Stoßwelle ist der Druck bis auf $\pm 3 \%$ konstant. Nach ca. 1,7 ms treffen die Verdünnungswellen in der Meßebene ein und beenden durch Druck- und Temperaturerniedrigung das Experiment. Damit ist die zur Verfügung stehende Meßzeit um 0,2 ms kürzer als die in Abschnitt (2.1.2) ideal berechnete Zeit. Das Intensitäts-Zeitprofil zeigt folgenden Verlauf: Nach einer kurzen Induktionsperiode von ca. $100 \mu$ s nimmt die Absorption zunächst stark zu und erreicht bis zum Ende der Meßzeit einen praktisch konstanten Wert. Kontrollexperimente mit reinem Argon als Testgas zeigen, daß bis auf die Schlierensignale das Laserlicht nicht abgeschwächt wurde.

Aus dem Absorptionssignal wurde mit Gleichung (2.10) das Rußausbeutezeitprofil $R A(t)$ berechnet und in Abbildung (3.2) dargestellt. Die Rußausbeute ist als Quotient aus Rußkonzentration und gesamter Kohlenstoffkonzentration definert.

$$
R A=\frac{[C]_{\mathrm{Ru} \beta}}{[C]_{\text {total }}}
$$

In der Fachliteratur wird häufig anstatt der Rußausbeute der Rußvolumenbruch $f_{v}$ als 


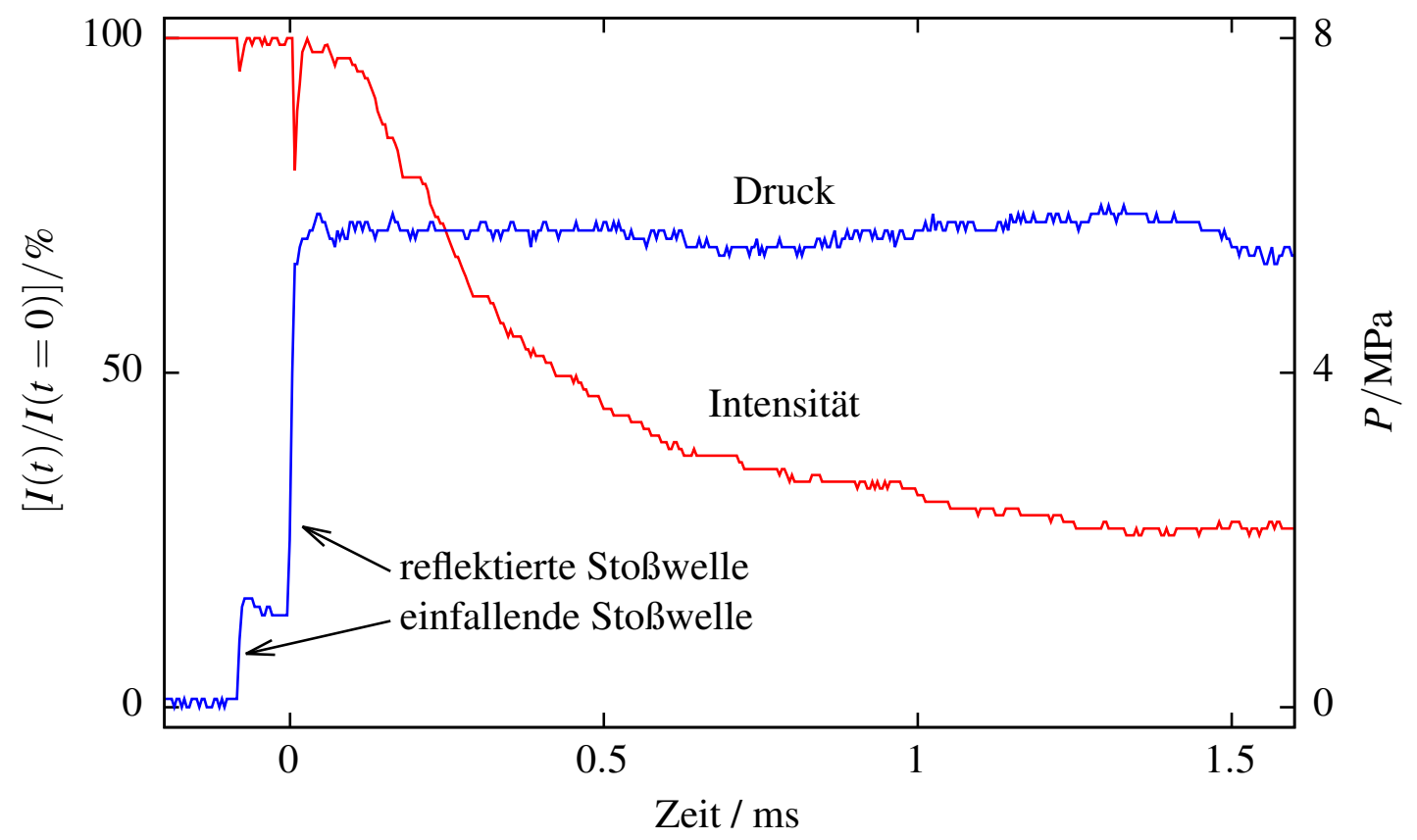

Abbildung 3.1: Das detektierte HeNe-Laserlicht-Intensitäts-Zeitprofil sowie das zugehörige Druckzeitprofil während einer Benzolpyrolyse hinter der reflektierten Stoßwelle. $T$ $=1890 \mathrm{~K}, P=5,5 \mathrm{MPa},[C]=0,84 \mathrm{~mol} / \mathrm{m}^{3}$.

Maß für die Rußmenge verwendet. Die Gleichung (3.2) gibt den Zusammenhang zwischen $f_{v}$ und $[C]_{\text {Ruß }}$ und wieder.

$$
f_{v}=[C]_{\mathrm{Ru} \beta} \cdot \frac{M_{C}}{\rho_{\mathrm{Ru} \beta}}
$$

Aus dem Schnittpunkt der Wendetangente mit der Zeitachse wurde in dieser Arbeit die Induktionszeit $\tau$ bestimmt. Für die Rußbildung in Abbildung (3.2) beträgt die Induktionszeit $80 \mu \mathrm{s}$. Nach der Induktionsperiode wächst die Rußausbeute mit einer maximalen Geschwindigkeit von $(\dot{R A})_{\max }=770 /$ s. Der Abschnitt des Rußprofils nach dem Wendepunkt kann gut durch eine Wachstumsfunktion pseudo-erster Ordnung wiedergegeben werden. Dieser Sachverhalt wurde zuerst in der Diplomarbeit von Geck [4] und später von Haynes et al [18] in einem empirisch gefundenen Gesetz, siehe Gleichung (3.3) dargestellt. Dort wurde das Rußmassenwachstum mit zwei Parametern $k_{f}$ und $f_{v \infty}$ beschrieben. $f_{v \infty}$ ist der Rußvolumenbruch am Ende des Rußbildungsprozesses und $k_{f}$ die ,formale Rußbildungsgeschwindigkeitskonstante“.

$$
\frac{\mathrm{d} f_{v}}{\mathrm{~d} t}=k_{f} \cdot\left(f_{v \infty}-f_{v}\right) \quad \text { und } \quad \frac{\mathrm{d}(R A)}{\mathrm{d} t}=k_{f} \cdot\left(R A_{\infty}-R A\right)
$$

Um aus den Rußvolumenbruchzeitprofilen die Parameter $k_{f}$ und $f_{v_{\infty}}$ zu erhalten, wurde in der Diplomarbeit von Tanke [19] zunächst $\dot{f}_{v}$ bestimmt und dann als Funktion von $f_{v}$ aufgetragen und der hintere Teil der Auftragung mit einer Geraden angepaßt. Aus der Steigung und dem Achsenabschnitt wurden die Parameter berechnet. Diese Methode enthält möglicherweise größere Fehlerquellen als die in dieser Arbeit angewendete direkte Anpassung der Rußausbeutezeitprofile mit Hilfe der integrierten Form von Gleichung (3.3). Deswegen wurden alle Rußvolumenbruchzeitprofile aus der Diplomarbeit nochmals ausgewertet und die Ergebnisse hier dargestellt. 


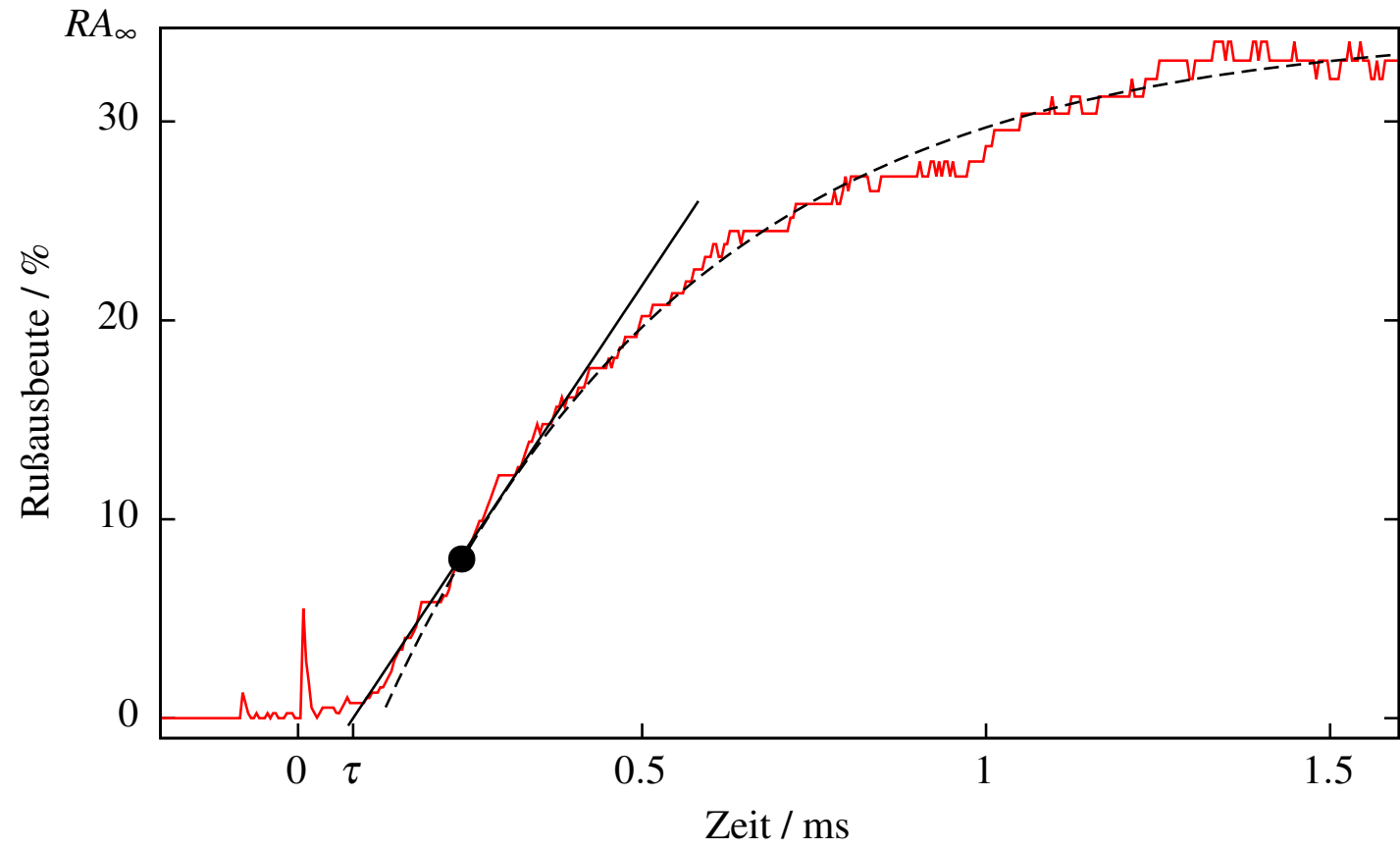

Abbildung 3.2: Aus dem Intensitäts-Zeitprofil der obigen Abbildung berechnetes Zeitprofil der Rußausbeute. Der ausgefüllte Kreis $(\bullet)$ markiert den Wendepunkt des Kurve und die Gerade die Wendetangente mit der Induktionszeit $\tau=80 \mu$ s und $(\dot{R A})_{\max }=518 / \mathrm{s}$. Die gestrichelte Kurve zeigt die ,gefittete“ Rußwachstumsfunktion pseudo-erster Ordnung mit den Parametern $t_{0}=120 \mu \mathrm{s}, k_{f}=2200 / \mathrm{s}$ und $R A_{\infty}=35 \%$, die die Rußbildung in der Massenwachstumsphase nach dem Wendepunkt gut beschreibt.

$\mathrm{Zu}$ Beginn der Rußbildung wird das Laserlicht größerer Wellenlänge weniger stark absorbiert als das Licht kleinerer Wellenlänge. Abbildung (3.3) zeigt den Vergleich der Rußausbeuten in einer Methanpyrolyse, die aus den Absorptions-Zeitprofilen bei den Wellenlängen 632,8 und $1064 \mathrm{~nm}$ berechnet wurden. Dazu wurde das Zeitprofil der Rußausbeute, das mit der Laserdiode bestimmt wurde $(R A(1064 \mathrm{~nm}))$, und das Zeitprofil des Quotienten aus den Rußausbeuten $(R A(632,8 \mathrm{~nm}) /(R A(1064 \mathrm{~nm}))$ aufgetragen. Im Idealfall ist dieser Quotient zu jedem Zeitpunkt nach dem Schlierensignal der reflektierten Stoßwelle gleich eins. Das ist jedoch nur zu Beginn der Pyrolyse und nach der Induktionsperiode der Fall. Innerhalb der Induktionsperiode wurde aus der Absorption des HeNe-Laser-Lichtes eine bis zu sechsfach höhere „Rußausbeute“ berechnet als aus der Absorption des Laserdioden-Lichtes. Zusätzlich sind Induktionszeiten und der Wendepunkt für beide RußZeitprofile eingetragen. Nach dem Wendepunkt sind die berechneten Rußausbeuten praktisch gleich groß.

In den folgenden drei Abschnitten werden die Abhängigkeiten der Induktionszeit, der formalen Rußbildungsgeschwindigkeitskonstante und der Rußausbeute von Temperatur, Kohlenstoffkonzentration, Druck und Kohlenwasserstoff vorgestellt.

\subsection{Induktionszeit}

Vor der Rußbildung wird stets eine Induktionsperiode beobachtet [1, 20, 4, 2], in der Rußvorläufer und evtl. erste Rußpartikel gebildet werden. In der Literatur wurden unterschiedliche Definitionen für die Induktionszeit beschrieben: 


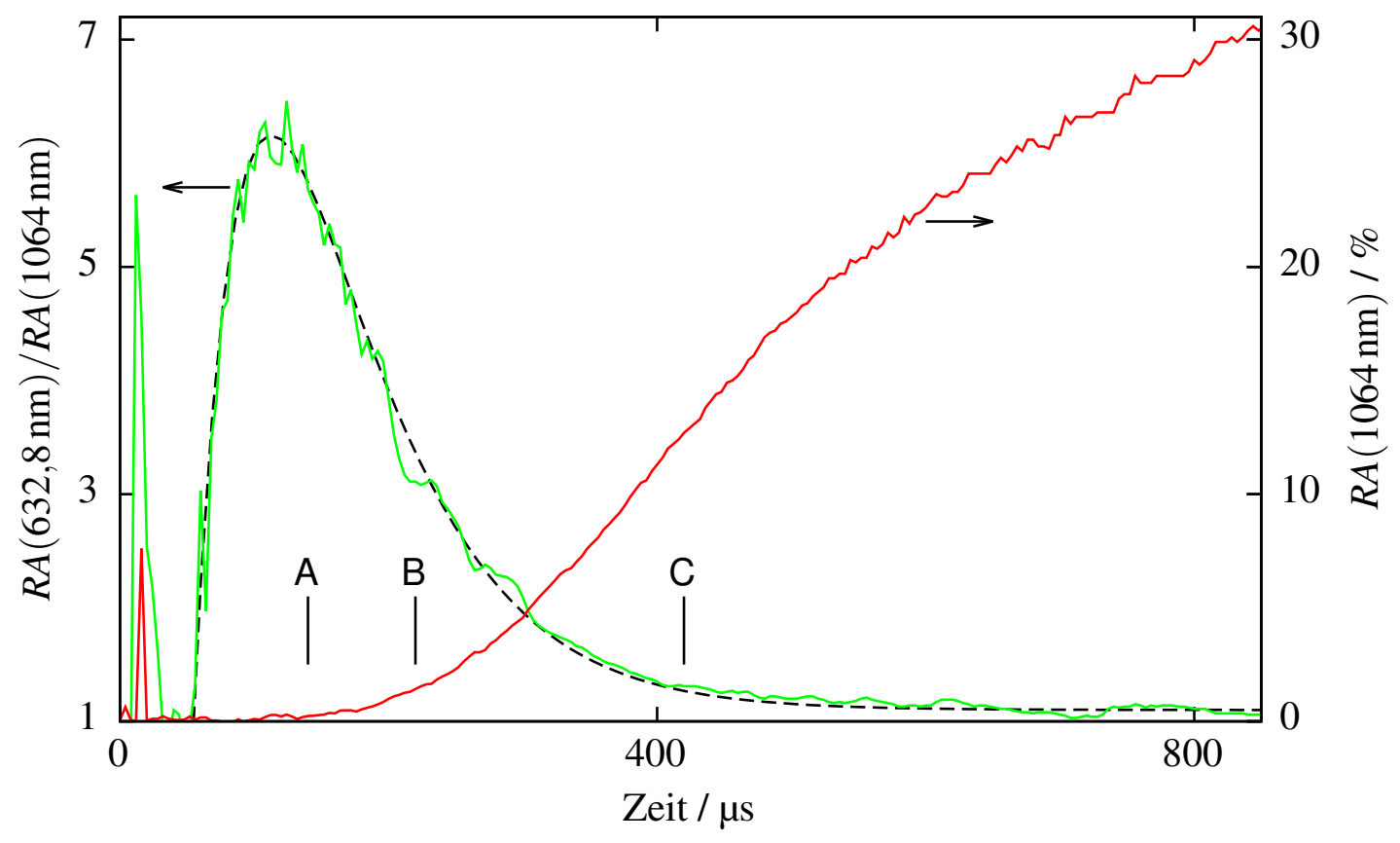

Abbildung 3.3: Zeitprofile der Rußausbeute, die aus dem Intensitäts-Zeitprofil des Laserdiodenlichtes berechnet wurde, und des Quotienten der Rußausbeuten $(R A(632,8 \mathrm{~nm}) /(R A(1064 \mathrm{~nm}))$. Zusätzlich sind die Induktionszeiten (A für $\lambda=$ $632,8 \mathrm{~nm}$ und $\mathrm{B}$ für $\lambda=1064 \mathrm{~nm}$ ) sowie des Wendepunktes $\mathrm{C}$ der Rußausbeutezeitprofile eingetragen. Die gestrichelte Kurve zeigt die beste Anpassung für die Entstehung von Zwischenprodukten. Pyrolysiert wurde $2 \%$ Methan bei einer Temperatur von $2150 \mathrm{~K}$ und einem Druck von 5,5 MPa, $[C]=6,1 \mathrm{~mol} / \mathrm{m}^{3}$.

- Abszissenschnittpunkt der Wendetangente im Rußkonzentrationsprofil [4]

- Zeitpunkt der ersten merklichen Lichtabsorption oder Emission [21, 20]

- Zeitpunkt der maximalen Krümmung des Absorptionsignals [2]

- Zeitpunkt, an dem die optische Dispersion den 1,15-fachen Wert des Grenzwertes für lange Reaktionszeiten unterschreitet [1]

- Zeitpunkt der sehr schnellen Zunahme des Rayleigh-Streulichtsignals [1]

Wegen des geringeren Fehlers wurde in dieser Arbeit der ersten Methode der Vorzug gegeben. Zum Auffinden der Wendetangente kann ein längerer und rauschärmerer Abschnitt des Rußausbeutezeitprofils genutzt werden als bei der zweiten, dritten und vierten Methode. Streulichtmessungen wurden nicht ausgeführt. Die Induktionszeiten werden in Abhängigkeit der Temperatur in Arrhenius-Diagrammen dargestellt.

\subsubsection{Benzol}

Abbildung (3.4) zeigt die Temperaturabhängigkeit der Induktionszeit für die Rußbildung anhand vier verschiedener Benzol-Argon Mischungen mit einem Benzolanteil von 200, 600, 2000 und 20000 ppm und einem konstanten Druck von $5 \mathrm{MPa}$. Das entspricht den mittleren Kohlenstoffkonzentrationen von 0,4, 1,0, 4,0 und $40 \mathrm{~mol} / \mathrm{m}^{3}$. Entsprechend dem 


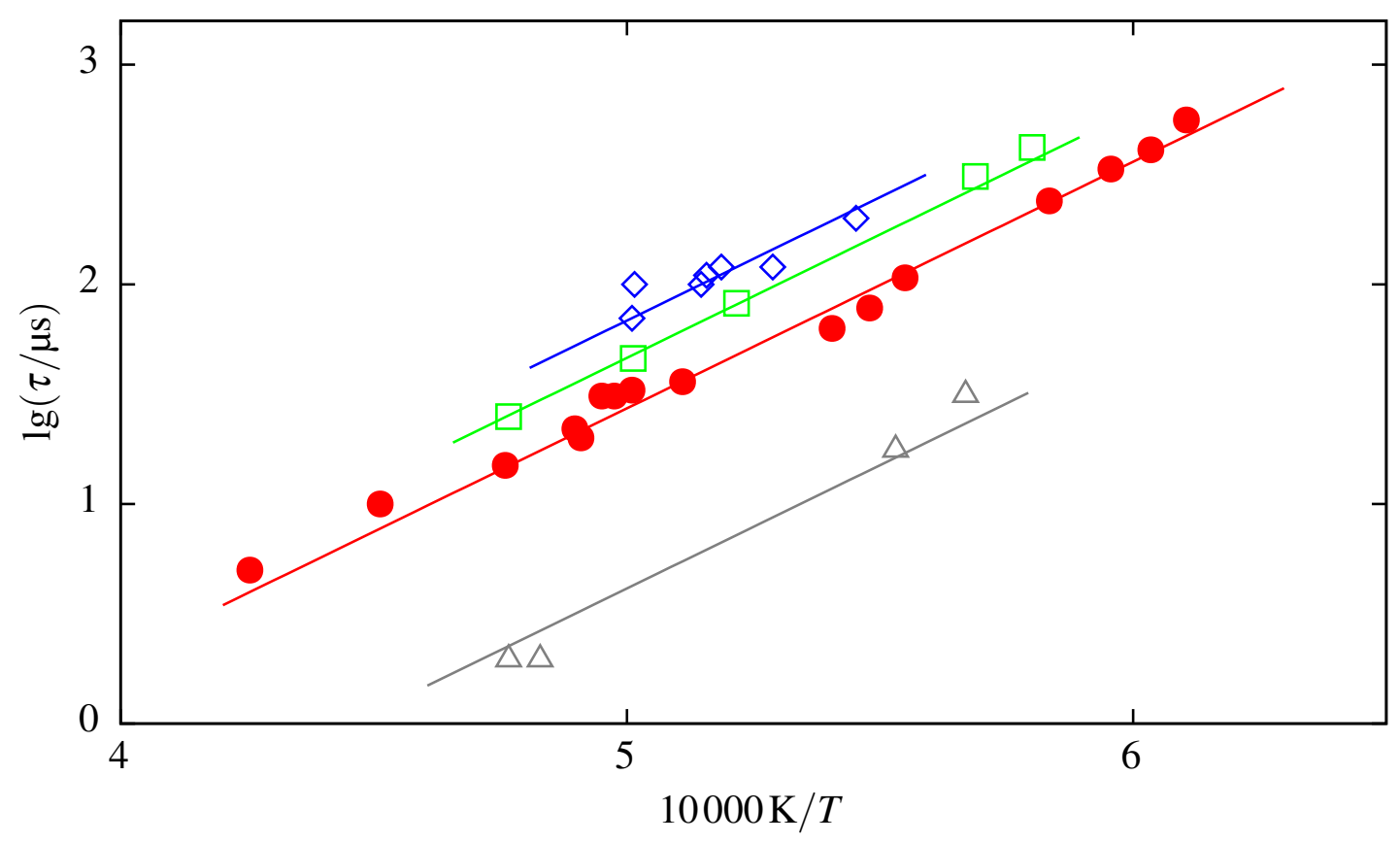

Abbildung 3.4: Temperaturabhängigkeit der Induktionszeit für vier verschiedene Benzolkonzentrationen und einem Druck von $5 \mathrm{MPa}$. Die Kohlenstoffkonzentrationen betragen: $\diamond: 0,4, \square: 1,0, \bullet: 4,0$ und $\triangle: 40 \mathrm{~mol} / \mathrm{m}^{3}$.

idealem Gasgesetz nahmen die Konzentrationen von niedrigen zu hohen Temperaturen stetig ab. Für die vier Benzolkonzentrationen erhält man vier parallele Geraden mit einer positiven Steigung, die einer scheinbaren Aktivierungsenergie von $215 \mathrm{~kJ} / \mathrm{mol}$ entspricht. Mit zunehmender Kohlenstoffkonzentration nehmen die präexponentiellen Faktoren stetig, von 166 auf 112,66 und $10 \times 10^{-12} \mathrm{~s}$ ab.

Die Abhängigkeit der Induktionszeit von der Kohlenstoffkonzentration läßt sich über zwei Größenordnungen mit dem in Gleichung (3.4) vorgeschlagenen Ansatz beschreiben:

$$
\tau \propto[C]^{-n} \quad \text { mit } n<1
$$

Der Exponent $n$ wird als frei wählbarer Parameter variiert. Die beste Anpassung der Meßwerte an den Ansatz aus Gleichung (3.4) erhält man mit $n=0,75$. Das Ergebnis ist in Abbildung (3.5) dargestellt. Aufgetragen sind die auf die Kohlenstoffkonzentration normierten Induktionszeiten in Abhängigkeit von der Temperatur. Darin enthalten sind auch die neu überarbeiteten Meßwerte aus der Diplomarbeit von Tanke [19]. Die Kohlenstoffkonzentration wurde von 0,35 bis $45 \mathrm{~mol} / \mathrm{m}^{3}$ variiert, der Druck von 0,5 bis $11 \mathrm{MPa}$. Man erhält eine um $7 \%$ größere scheinbare Aktivierungsenergie von $229 \mathrm{~kJ} / \mathrm{mol}$, weil sich die Kohlenstoffdichte mit zunehmender Temperatur verringerte.

Ein Druckeinfluß auf die Induktionszeit ist nicht vorhanden. Zusammenfassend wird die Abhängigkeit von Temperatur, Kohlenstoffkonzentration und Druck innerhalb des untersuchten Bereiches auf die Induktionszeit der Rußbildung in der Pyrolyse von Benzol durch Gleichung (3.5) beschrieben.

$$
\tau / \mathrm{s}=6,5 \cdot 10^{-11} \cdot P^{0} \cdot \exp \left(\frac{229 \mathrm{~kJ} / \mathrm{mol}}{R T}\right) \cdot\left(\frac{[C]}{\mathrm{mol} / \mathrm{m}^{3}}\right)^{-0,75}
$$




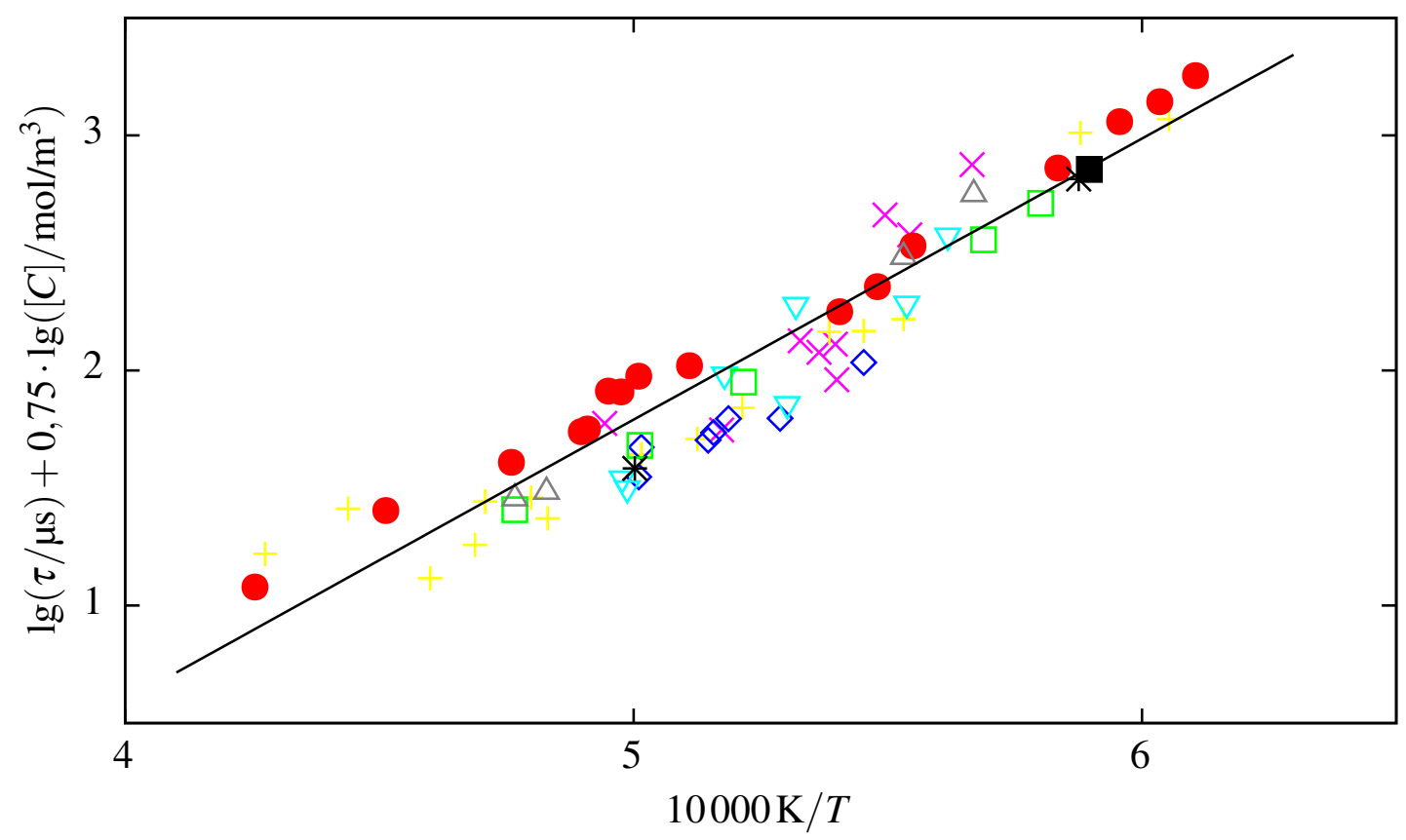

Abbildung 3.5: Temperaturabhängigkeit der auf die Kohlenstoffkonzentration normierten Induktionszeit für die Rußbildung in der Benzolpyrolyse. Die Drücke und Kohlenstoffkonzentration betragen: $P=0,5 \mathrm{MPa}, \times: 0,4 \mathrm{~mol} / \mathrm{m}^{3} . P=2,5 \mathrm{MPa},+: 1,1 \mathrm{~mol} / \mathrm{m}^{3}$. $P=5 \mathrm{MPa}, \diamond: 0,4, \nabla: 0,8, \square: 1,0, \bullet: 4,0$ und $\triangle: 40 \mathrm{~mol} / \mathrm{m}^{3} . P=11 \mathrm{MPa}, *: 0,4$ und п: $0,8 \mathrm{~mol} / \mathrm{m}^{3}$.

\subsubsection{Phenylacetylen}

Als zweiter Vertreter der Aromaten wurde Phenylacetylen mit einem Molenbruch von $500 \mathrm{ppm}$ bei $5,7 \mathrm{MPa}$ pyrolysiert. Die mittlere Kohlenstoffkonzentration betrug $1,4 \mathrm{~mol} / \mathrm{m}^{3}$. Den Temperatureinfluß auf die Induktionszeit zeigt Abbildung (3.6). Man erhält annähernd dieselbe scheinbare Aktivierungsenergie $(215 \mathrm{~kJ} / \mathrm{mol})$ wie bei Benzol.

\subsubsection{Acetylen}

Die Temperaturabhängigkeit der Induktionszeit für die Rußbildung bei der Acetylenpyrolyse wurde bei drei verschiedenen mittleren Kohlenstoffkonzentrationen $([C]=0,9,1,7$ und $\left.3,8 \mathrm{~mol} / \mathrm{m}^{3}\right)$ und zwei Drücken $(2,6$ und 5,7 MPa) untersucht. Für die unterschiedlichen Kohlenstoffkonzentrationen erhält man Geraden mit gleicher Steigung und verschiedenen präexponentiellen Faktoren. Die scheinbare Aktivierungsenergie ist $216 \mathrm{~kJ} / \mathrm{mol}$. Das ist praktisch die gleiche Temperaturabhängigkeit wie die der untersuchten Aromaten. Die Halbierung des Druckes hat keinen Einfluß auf die Induktionszeit. Mit zunehmender Kohlenstoffkonzentration sinkt die Induktionszeit. Die Abhängigkeit der Induktionszeit von der Kohlenstoffkonzentration wird mit dem Ansatz in Gleichung (3.4) beschrieben. Der Exponent $n$ hat den gleichen Wert wie bei der Benzolpyrolyse, $n=0,75$. In Abbildung (3.8) sind die auf die Kohlenstoffkonzentration normierten Induktionszeiten in Abhängigkeit von der Temperatur aufgetragen. Alle Messungen wurden mit einer Geraden angepaßt. Zusammenfassend wird die Abhängigkeit von Temperatur, Kohlenstoffkonzentration und Druck auf die Induktionszeit der Rußbildung in der Pyrolyse von Acetylen durch Gleichung (3.6) beschrieben. 


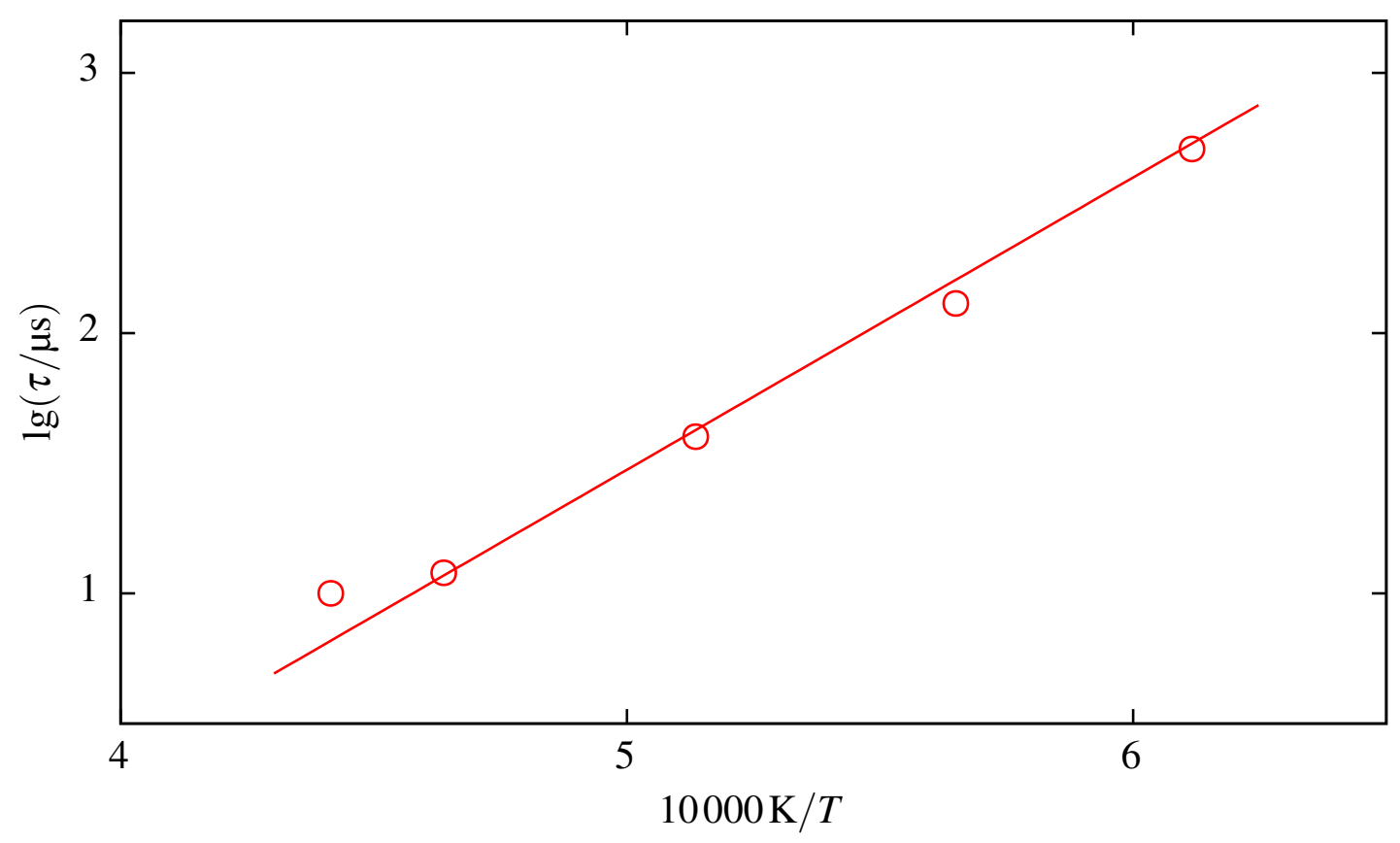

Abbildung 3.6: Temperaturabhängigkeit der Rußbildungs-Induktionszeit von Phenylacetylen bei einer Kohlenstoffkonzentration von 1,4 mol/ $\mathrm{m}^{3}$ und einem Druck von 5,7 MPa.

$$
\tau / \mathrm{s}=2,9 \cdot 10^{-10} \cdot P^{0} \cdot \exp \left(\frac{228 \mathrm{~kJ} / \mathrm{mol}}{R T}\right) \cdot\left(\frac{[C]}{\mathrm{mol} / \mathrm{m}^{3}}\right)^{-0,75}
$$

\subsubsection{Ethylen}

Die Temperaturabhängigkeit der Induktionszeit für die Rußbildung in der Ethylenpyrolyse wurde hinter reflektierten Stoßwellen im Druckbereich zwischen 0,6 und $11 \mathrm{MPa}$ untersucht. Die Kohlenstoffkonzentration wurde von 3,9 bis $12 \mathrm{~mol} / \mathrm{m}^{3}$ variiert. Dabei wurde im Rahmen der Meßgenauigkeit weder eine Druckabhängigkeit noch eine Konzentrationsabhängigkeit der Induktionszeit festgestellt. In Abbildung (3.9) werden alle Meßwerte mit einer Geraden angepaßt. Die scheinbare Aktivierungsenergie beträgt $216 \mathrm{~kJ} / \mathrm{mol}$. Da in der Literatur eine Abhängigkeit der Induktionszeit von der Ethylenkonzentration angegeben wurde [4], wurde mit Hilfe von Grenzgeraden ein maximaler Wert für den Exponenten $n$ aus Gleichung (3.4) abgeschätzt. Es ergab sich ein oberer Grenzwert für $n$ von 0,4. Abbildung (3.10) zeigt Induktionszeiten der Rußbildung für ein $3 \%$ Ethylen enthaltendes Testgas hinter einfallenden Stoßwellen bei einem Druck von 0,53 MPa und einer Kohlenstoffkonzentration von $2,0 \mathrm{~mol} / \mathrm{m}^{3}$. Der Betrag der scheinbaren Aktivierungsenergie von $85 \mathrm{~kJ} / \mathrm{mol}$ ist um $60 \%$ kleiner als die hinter reflektierten Stoßwellen bestimmte Temperaturabhängigkeit.

\subsubsection{Methan}

Die Temperaturabhängigkeit der Induktionszeit für die Rußbildung bei der Methanpyrolyse wurde im Druckbereich zwischen 2,5 und $24 \mathrm{MPa}$ untersucht. Die Kohlenstoffkonzentration wurde von $1,7 \mathrm{bis} 28 \mathrm{~mol} / \mathrm{m}^{3}$ variiert. In Abbildung (3.11) werden 


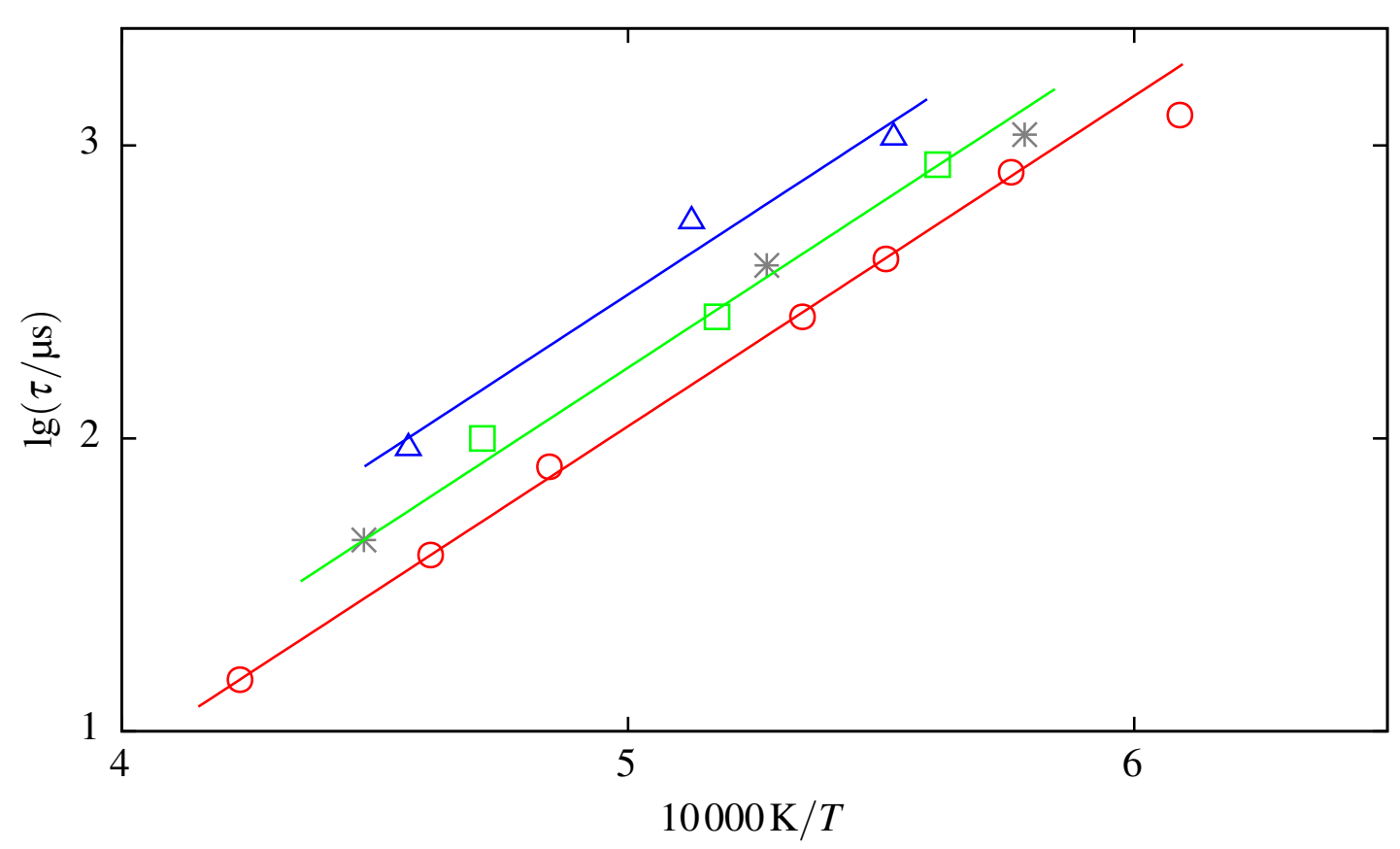

Abbildung 3.7: Temperaturabhängigkeit der Induktionszeit für drei verschiedene Acetylenkonzentrationen und zwei unterschiedlichen Drücken. o: $5,7 \mathrm{MPa}, 3,8 \mathrm{~mol} / \mathrm{m}^{3}$, $\square: 5,7 \mathrm{MPa}, 1,7 \mathrm{~mol} / \mathrm{m}^{3}, \triangle: 5,7 \mathrm{MPa}, 0,9 \mathrm{~mol} / \mathrm{m}^{3}, *: 2,6 \mathrm{MPa}, 1,7 \mathrm{~mol} / \mathrm{m}^{3}$. Die angegebenen Konzentrationen beziehen sich auf die C-Atome. Die Meßwerte bei unterschiedlichem Druck aber gleicher Kohlenstoffkonzentration ( $\square$ und $*$ ) wurden mit ein und derselben Geraden angepaßt.

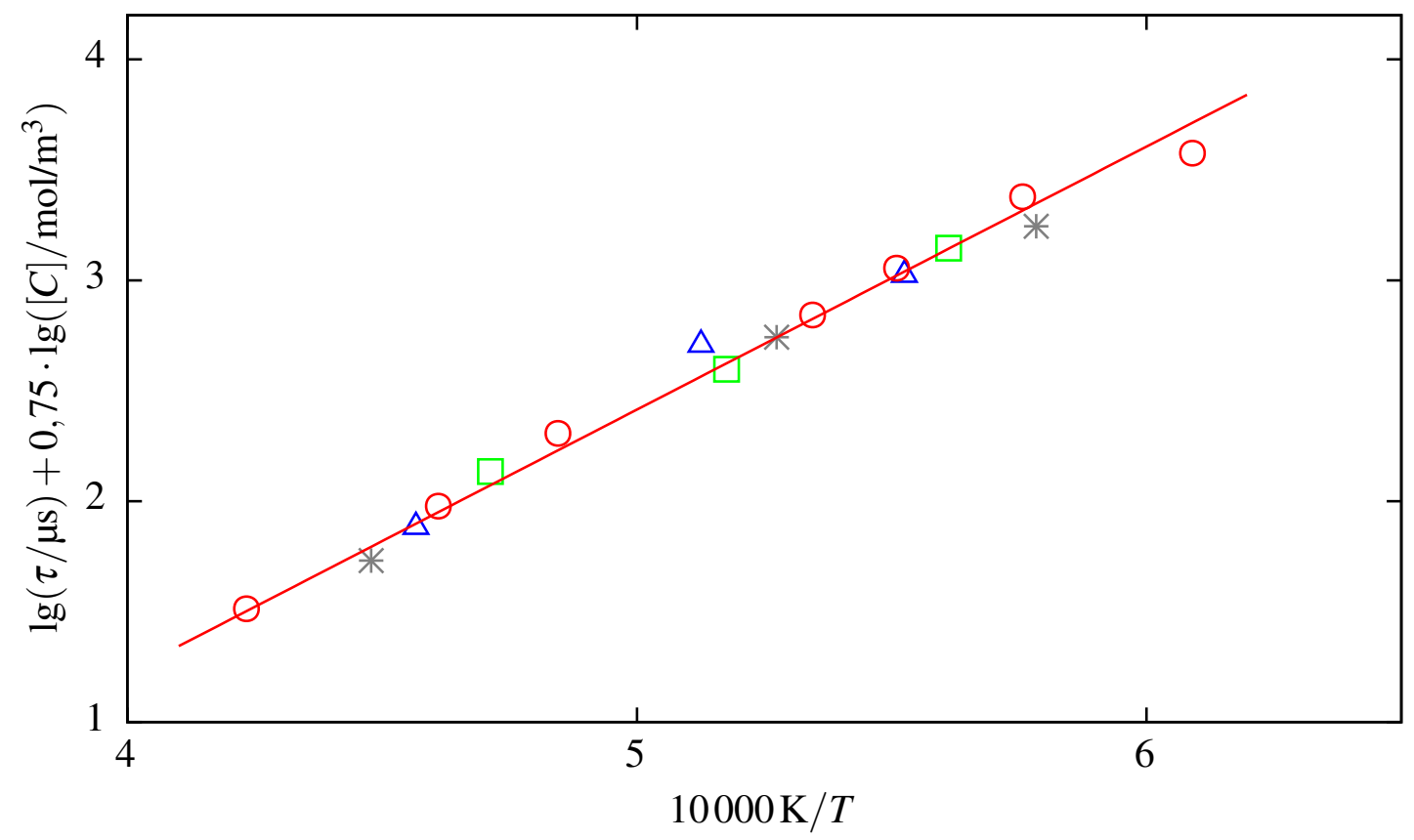

Abbildung 3.8: Temperaturabhängigkeit der auf die Kohlenstoffkonzentration normierten Induktionszeit für die Rußbildung in der Acetylenpyrolyse. (Legende: siehe obige Abbildung). 


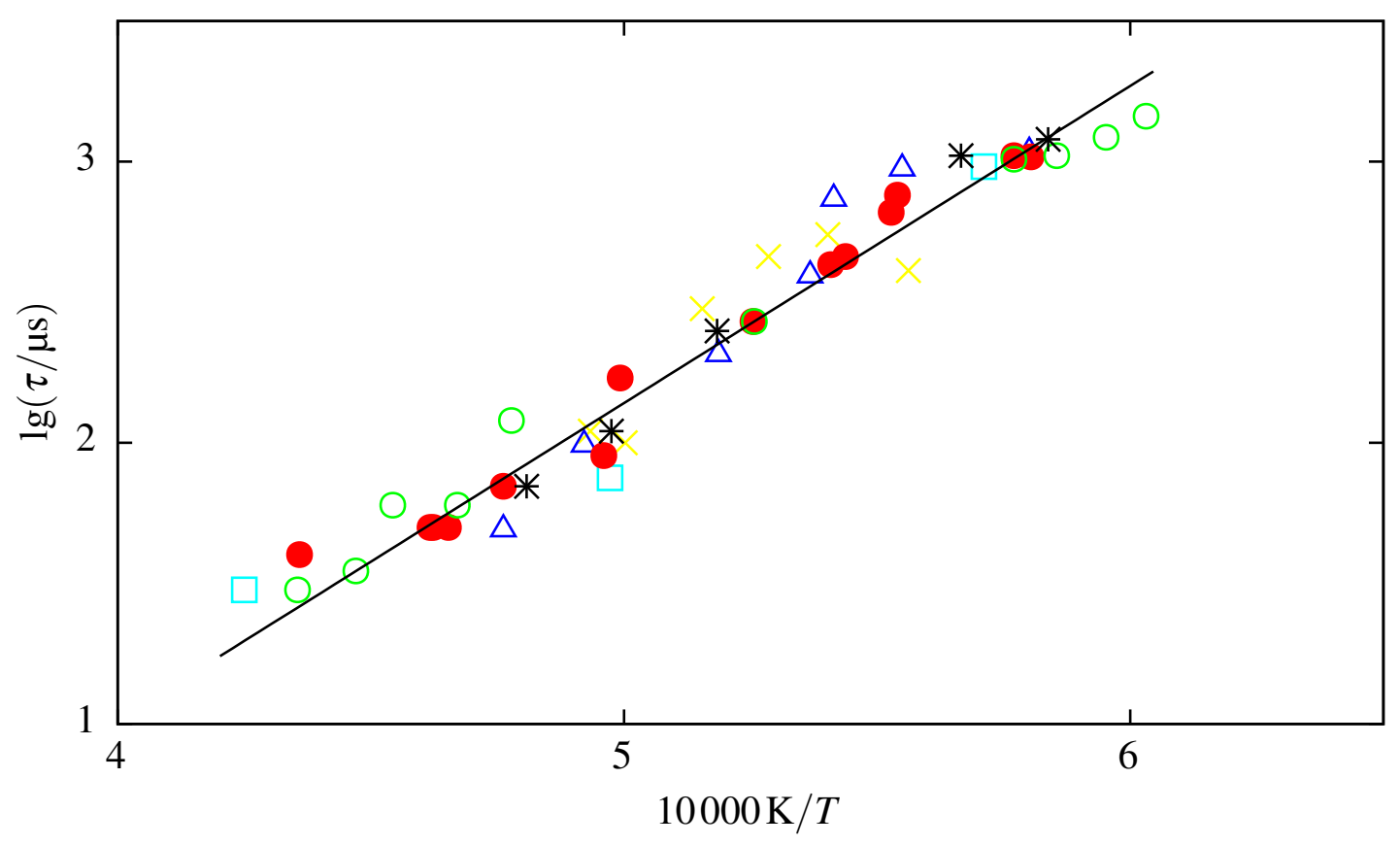

Abbildung 3.9: Temperaturabhängigkeit der Induktionszeit für verschiedene Ethylenkonzentrationen und Drücke. Die angegebenen Konzentrationen beziehen sich auf die C-Atome. $\times: 0,6 \mathrm{MPa}, 3,9 \mathrm{~mol} / \mathrm{m}^{3} \triangle: 2,5 \mathrm{MPa}, 4,0 \mathrm{~mol} / \mathrm{m}^{3} \square: 2,5 \mathrm{MPa}, 12 \mathrm{~mol} / \mathrm{m}^{3}$ •: $5,0 \mathrm{MPa}, 4,2 \mathrm{~mol} / \mathrm{m}^{3}$ ○: $5,0 \mathrm{MPa}, 7,0 \mathrm{~mol} / \mathrm{m}^{3}$ und $*: 11 \mathrm{MPa}, 4,2 \mathrm{~mol} / \mathrm{m}^{3}$.

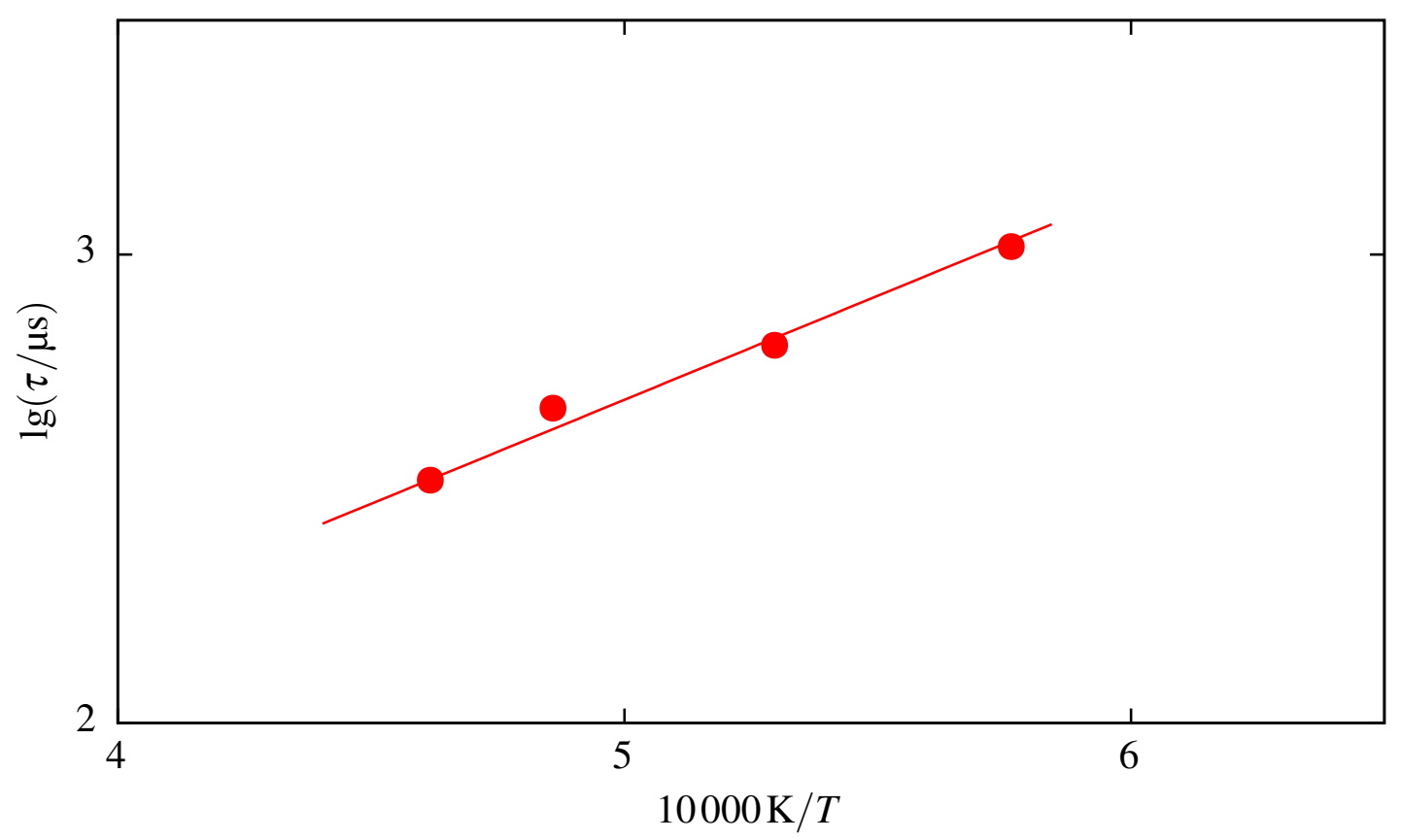

Abbildung 3.10: Temperaturabhängigkeit der Induktionszeit für Ethylen hinter einfallenden Stoßwellen; $P=0,53 \mathrm{MPa}$ und $[C]=2,0 \mathrm{~mol} / \mathrm{m}^{3}$. 


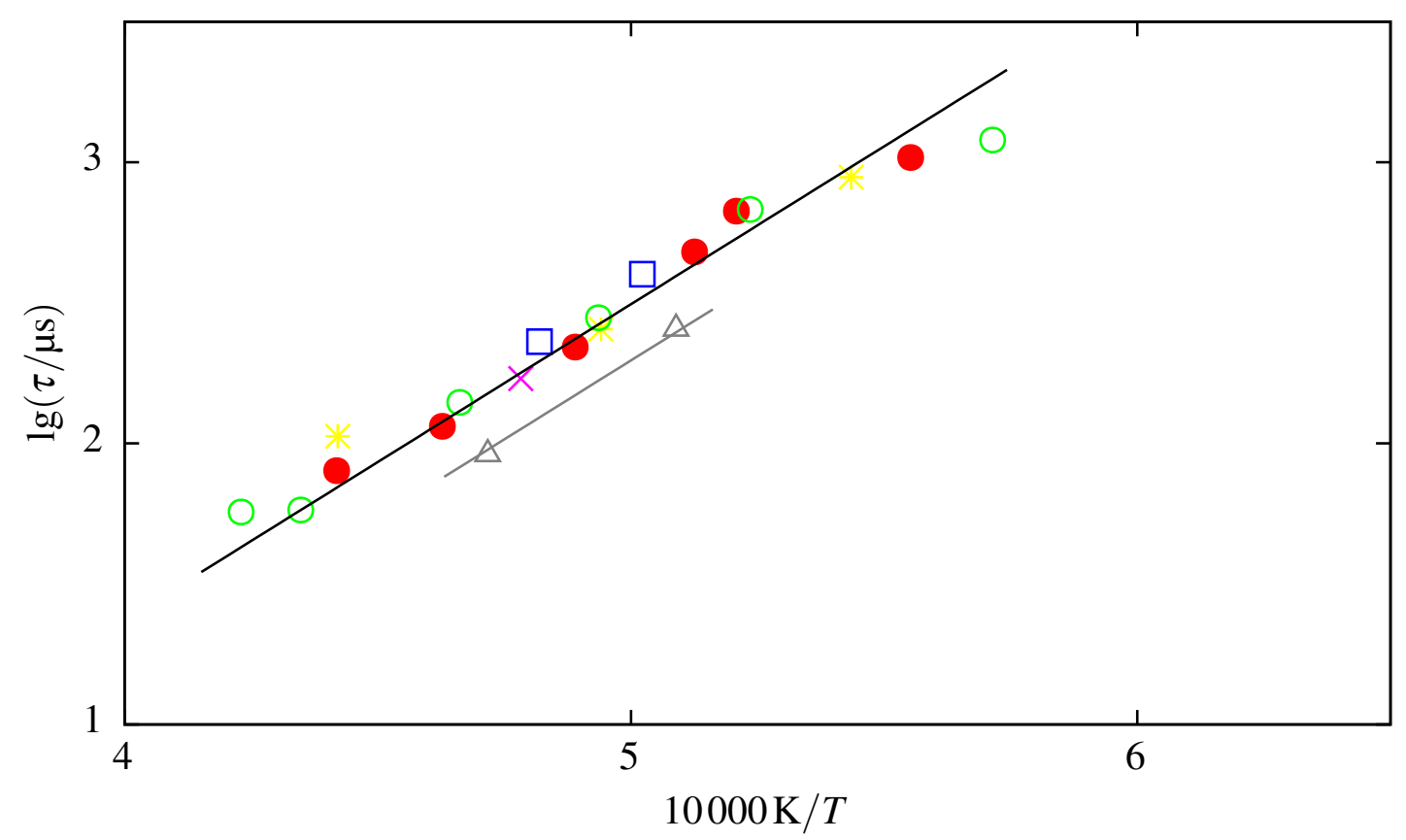

Abbildung 3.11: Temperaturabhängigkeit der Induktionszeit für verschiedene Methankonzentrationen und Drücke. $*: 2,5 \mathrm{MPa}, 3,0 \mathrm{~mol} / \mathrm{m}^{3} \bullet: 5,5 \mathrm{MPa}, 3,4 \mathrm{~mol} / \mathrm{m}^{3} \times: 12 \mathrm{MPa}$, $3,4 \mathrm{~mol} / \mathrm{m}^{3} \square: 5,5 \mathrm{MPa}, 1,7 \mathrm{~mol} / \mathrm{m}^{3}$ ○: $5,5 \mathrm{MPa}, 6,4 \mathrm{~mol} / \mathrm{m}^{3}$ und $\triangle: 24 \mathrm{MPa}, 28 \mathrm{~mol} / \mathrm{m}^{3}$.

fast alle Meßwerte mit einer Geraden angepaßt. Die scheinbare Aktivierungsenergie beträgt $215 \mathrm{~kJ} / \mathrm{mol}$. Bis auf die Experimente bei $24 \mathrm{MPa}$ wurde weder eine Druckabhängigkeit noch eine Konzentrationsabhängigkeit der Induktionszeit festgestellt. Ob die ca. 50-prozentige Abnahme der Induktionszeit bei maximalen Drücken auf den Einfluß des Druckes oder der sehr hohen Kohlenstoffkonzentration zurückzuführen ist, muß hier offen bleiben.

\subsection{6 n-Hexan}

Abbildung (3.12) zeigt die Temperaturabhängigkeit für die Induktionszeit der Rußbildung für hohe Kohlenstoffdichten $\left([C]=35 \mathrm{~mol} / \mathrm{m}^{3}\right)$ bei 5,0 MPa. Zusätzlich sind die neu überarbeiteten Meßwerte aus der Diplomarbeit von Tanke [19] mit aufgenommen. Die Kohlenstoffdichte wurde konstant auf $5,3 \mathrm{~mol} / \mathrm{m}^{3}$ gehalten, der Druck von 2 bis $10 \mathrm{MPa}$ variiert. Auffällig ist die relativ große Streuung der Meßwerte aus der Diplomarbeit, die nicht mit den unterschiedlichen Drücken erklärt werden kann. Obwohl die Kohlenstoffkonzentration um mehr als den Faktor sechs erhöht wurde, änderte sich die Induktionszeit nicht. Man erhält eine scheinbare Aktivierungsenergie von $215 \mathrm{~kJ} / \mathrm{mol}$.

\subsubsection{Vergleich der Kohlenwasserstoffe}

Zusammenfassend ist die Abhängigkeit der Induktionszeit der Rußbildung von der Temperatur und vom eingesetzten Kohlenwasserstoff in den Abbildungen (3.13), (3.14) und in Tabelle (3.1) dargestellt. Abbildung (3.13) zeigt die Induktionszeiten für Acetylen, Benzol und Phenylacetylen bei einer mittleren Kohlenstoffdichte von $1,4 \mathrm{~mol} / \mathrm{m}^{3}$. Im Vergleich zu Benzol setzt die Rußbildung in der Pyrolyse von Phenylacetylen etwas früher und bei 


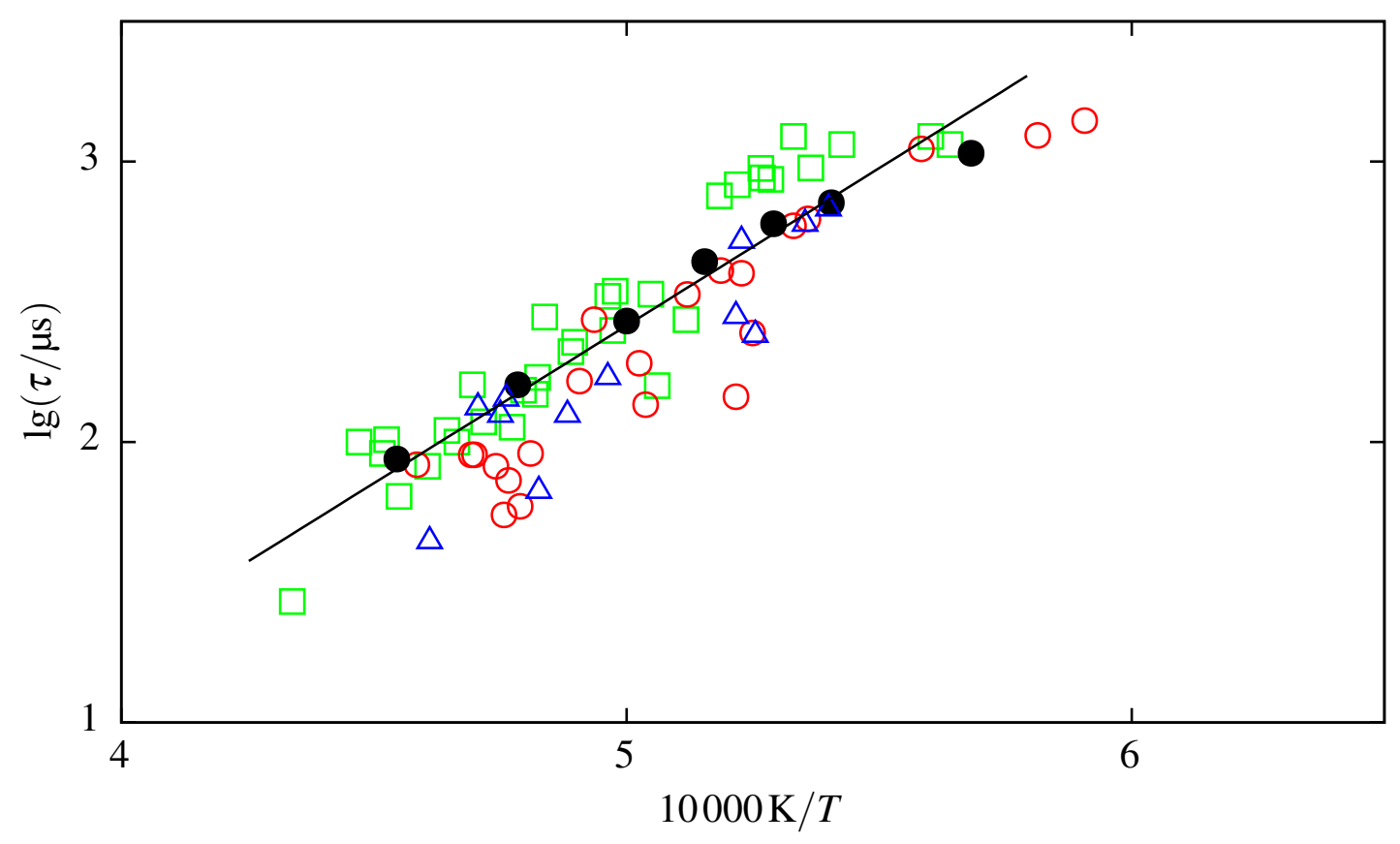

Abbildung 3.12: Temperaturabhängigkeit der Induktionszeit für verschiedene Drücke und Hexan=Konzentrationen: $[C]=5,3 \mathrm{~mol} / \mathrm{m}^{3}, \square: 2,5 \circ: 6$ und $\triangle: 10 \mathrm{MPa} .[C]$ $=35 \mathrm{~mol} / \mathrm{m}^{3}$, •: $5 \mathrm{MPa}$.

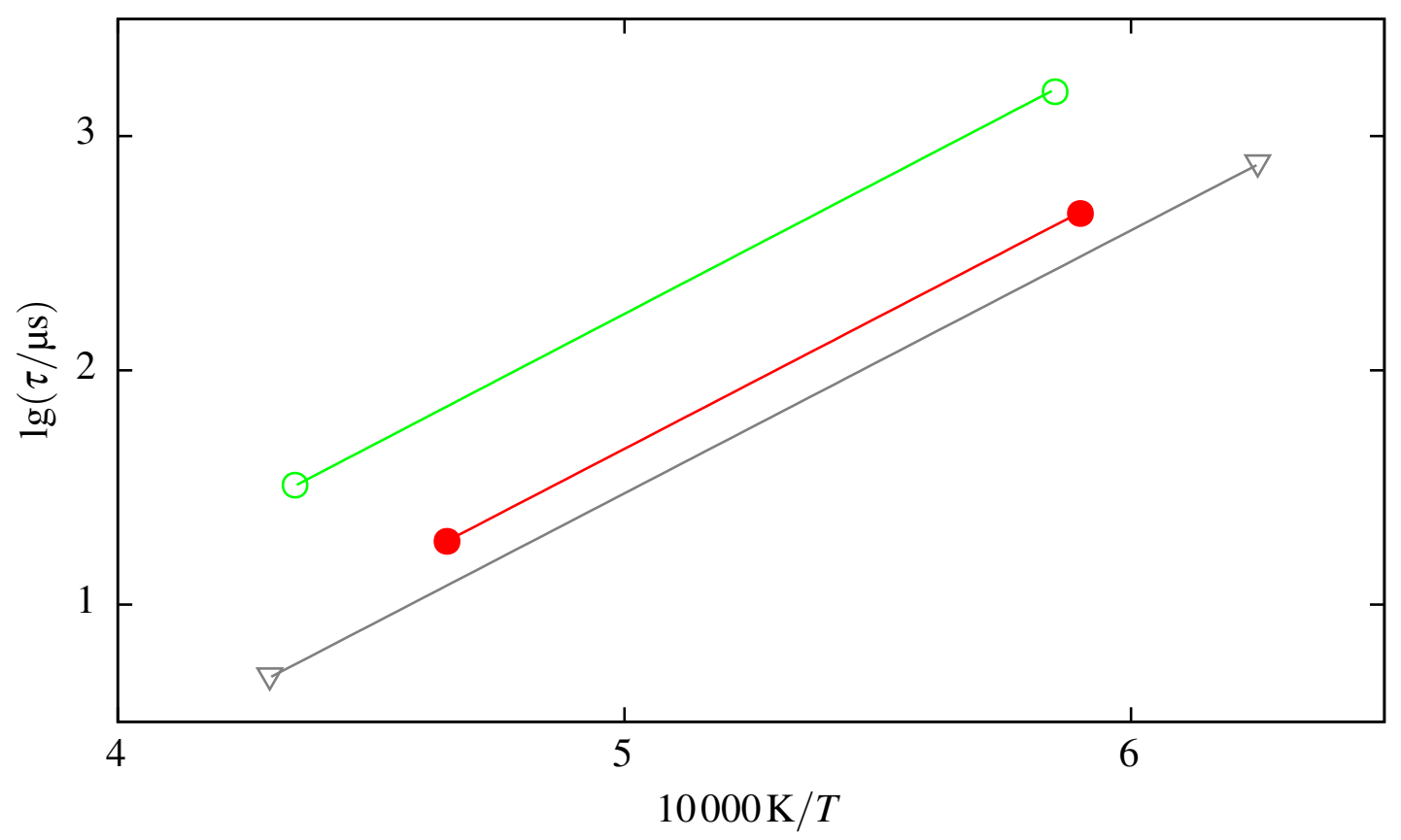

Abbildung 3.13: Vergleich der Induktionszeiten für die Rußbildung in Abhängigkeit der Temperatur für verschiedene Kohlenwasserstoffe bei annähernd konstanter Kohlenstoffdichte. Die angegebenen Konzentrationen beziehen sich auf die C-Atome. ०: Acetylen $1,7 \mathrm{~mol} / \mathrm{m}^{3} \bullet$ : Benzol 1,1 $\mathrm{mol} / \mathrm{m}^{3}$ und $\nabla$ : Phenylacetylen $1,4 \mathrm{~mol} / \mathrm{m}^{3}$. 


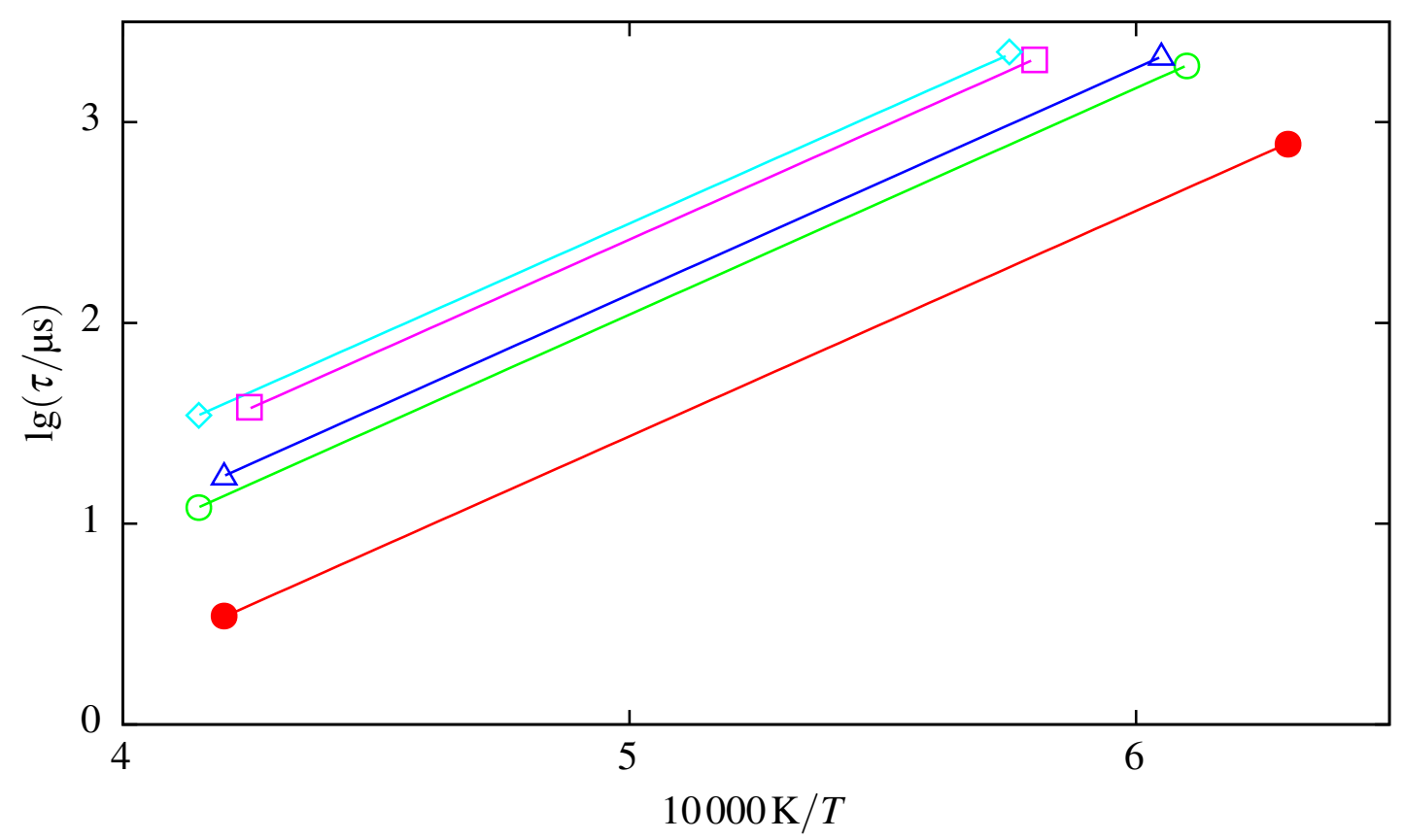

Abbildung 3.14: Vergleich der Induktionszeiten für die Rußbildung in Abhängigkeit der Temperatur für verschiedene Kohlenwasserstoffe. Die angegebenen Konzentrationen beziehen sich auf die C-Atome. $\bullet$ : Benzol $4,0 \mathrm{~mol} / \mathrm{m}^{3} \circ$ : Acetylen $3,8 \mathrm{~mol} / \mathrm{m}^{3} \triangle$ : Ethylen 3,9 bis $12 \mathrm{~mol} / \mathrm{m}^{3} \diamond$ : Methan 1,7 bis $6,4 \mathrm{~mol} / \mathrm{m}^{3}$ und $\square$ : n-Hexan 5,3 bis $35 \mathrm{~mol} / \mathrm{m}^{3}$.

Acetylen später ein. In Abbildung (3.14) werden die Kohlenwasserstoffe untereinander verglichen, deren Induktionszeiten nicht oder nur wenig von der Kohlenstoffdichte abhängen (Methan, n-Hexan und Ethylen). Zusätzlich sind die Induktionszeiten von Acetylen und Benzol bei einer vergleichbaren Kohlenstoffkonzentrationen von $4 \mathrm{~mol} / \mathrm{m}^{3}$ eingetragen. Die Induktionszeiten der Alkane Methan und n-Hexan sind gleich lang. Im Vergleich dazu sind die Induktionszeiten von Ethylen und Acetylen um den Faktor 2,5 kürzer, die von Benzol um den Faktor 10 kürzer. Allgemein gilt für die Abhängigkeit der Induktionszeit von Temperatur und Kohlenstoffkonzentration die Gleichung (3.7). Die zugehörigen Arrhenius-Parameter und der Exponent $n$ sind in Tabelle (3.1) für die verschiedenen Kohlenwasserstoffe angegeben.

$$
\tau=A \cdot \exp \left(E_{\text {Ind }} / R T\right) \cdot \frac{P^{0}}{[C]^{n}}
$$

\subsubsection{Messungen mit der Laserdiode}

Die Induktionszeiten, die mit der Laserdiode bei höherer Wellenlänge bestimmt wurden, waren stets länger als die mit dem HeNe-Laser gemessenen Werte. Das heißt, daß der Quotient der Induktionszeiten stets größer eins ist $(\tau(1064 \mathrm{~nm}) / \tau(632,8 \mathrm{~nm})>1)$. Für Methan ist in Abbildung (3.15) die Temperaturabhängigkeit dieses Quotienten dargestellt. Mit steigender Temperatur nimmt der Quotient von 1,2 bei $1900 \mathrm{~K}$ auf 1,8 bei $2300 \mathrm{~K}$ zu. 


\begin{tabular}{lccccc}
\hline Edukt & $T / \mathrm{K}$ & {$[C] / \frac{\mathrm{mol}}{\mathrm{m}^{3}}$} & $-\lg (A / \mathrm{s})$ & $E_{\text {Ind }} / \frac{\mathrm{kJ}}{\mathrm{mol}}$ & $n$ \\
\hline Benzol & 1600 bis 2400 & 0,4 bis 40 & 10,19 & 229 & 0,75 \\
Phenylacetylen & 1600 bis 2200 & 1,4 & 10,13 & 215 & - \\
Acetylen & 1700 bis 2400 & 0,9 bis 3,8 & 9,54 & 228 & 0,75 \\
Ethylen & 1650 bis 2300 & 3,9 bis 12 & 9,49 & 216 & $<0,4$ \\
Methan & 1750 bis 2400 & 1,7 bis 6,4 & 9,12 & 215 & 0 \\
n-Hexan & 1750 bis 2300 & 5,3 bis 35 & 9,20 & 215 & 0 \\
\hline
\end{tabular}

Tabelle 3.1: Die drei Parameter zur Berechnung von Rußbildungs-Induktionszeiten verschiedener Kohlenwasserstoffe: Arrhenius-Vorfaktor $A$, scheinbare Aktivierungsenergie $E_{\text {Ind }}$ und Abhängigkeit von der Kohlenstoffkonzentration $n$. Die Konzentrationsabhängigkeit von Phenylacetylen wurde nicht untersucht.

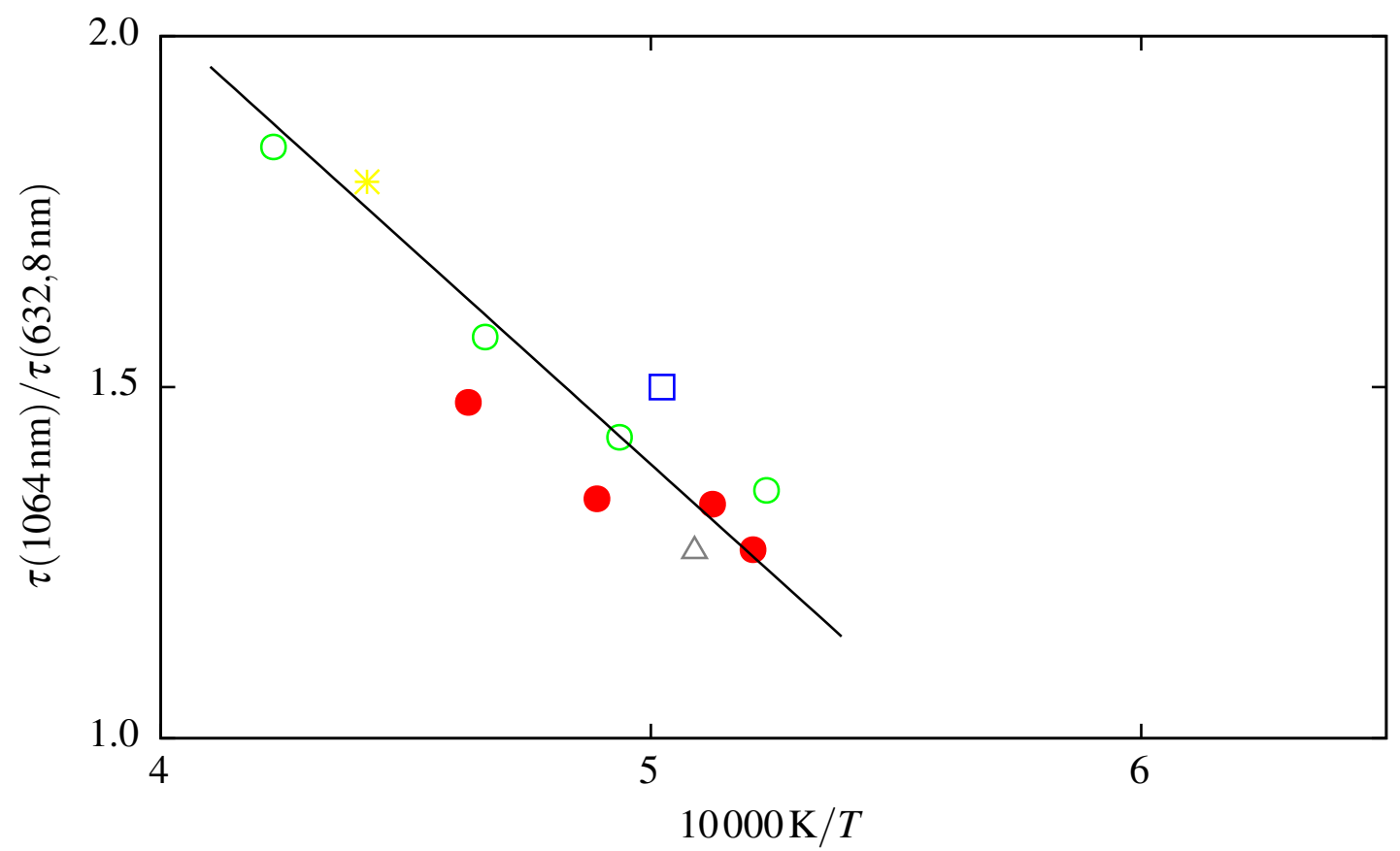

Abbildung 3.15: Quotient der Induktionszeiten $(\tau(1064 \mathrm{~nm}) / \tau(632,8 \mathrm{~nm}))$ als Funktion der Temperatur in der Methanpyrolyse. *: 2,5 MPa, 3,0 mol $/ \mathrm{m}^{3} \bullet: 5,5 \mathrm{MPa}, 3,4 \mathrm{~mol} / \mathrm{m}^{3}$ $\square: 5,5 \mathrm{MPa}, 1,7 \mathrm{~mol} / \mathrm{m}^{3}$ ○: $5,5 \mathrm{MPa}, 6,4 \mathrm{~mol} / \mathrm{m}^{3}$ und $\triangle: 24 \mathrm{MPa}, 28 \mathrm{~mol} / \mathrm{m}^{3}$. 


\subsection{Rußmassenwachstum}

Nach der Induktionsperiode beginnt das Rußmassenwachstum. Wie in Abbildung (3.3) gezeigt wurde, ist nach dem Wendepunkt die Höhe der detektierten Rußausbeute unabhängig von den hier benutzten Laserlichtwellenlängen. Daher sind die Ergebnisse für die formale Rußbildungsgeschwindigkeitskonstante $k_{f}$, die aus den HeNe-Laser Absorptions-Zeitprofilen gewonnen wurden, fast gleich mit den Ergebnissen, die mit der Laserdiode erzielt wurden. Mit dem auf Seite 16 eingeführten Wachstumsgesetz pseudo-erster Ordnung (Gleichung (3.3)) lassen sich die Rußausbeutezeitprofile nach dem Wendepunkt gut beschreiben. Da es problematisch war aus dem Rußausbeute-Zeitprofil $R A(t)$ die erste Ableitung nach der Zeit $(\dot{R A})$ zu bilden, wurde in dieser Arbeit die integrierte Form des Wachstumsgesetzes verwendet, siehe Gleichung (3.8).

$$
R A(t)=R A_{\infty} \cdot\left\{1-\exp \left[k_{f} \cdot\left(t_{0}-t\right)\right]\right\}
$$

Der leicht bestimmbare Parameter $t_{0}$ ist eine Zeit, die zumeist etwas länger als die Induktionszeit $\tau$ ist. Dieses Verfahren wurde im folgenden auf alle Rußausbeute-Zeitprofile der untersuchten Brennstoffe angewendet. Für Ethylen wurde von Geck [4] gezeigt, daß die Massenwachstumskonstante $k_{f}$ direkt proportional zur Kohlenstoffdichte $[C]$ ist:

$$
k_{f} \propto[C]^{n} \quad \text { mit: } \quad n=1
$$

Dieser Sachverhalt wurde in dieser Arbeit auch für Benzol, Acetylen, Ethylen und Methan bestätigt. Deshalb wird nur einmal exemplarisch für Benzol die Massenwachstumskonstante in Abhängigkeit der Temperatur dargestellt und für alle weiteren Kohlenwasserstoffe gleich die normierte Massenwachstumskonstante $\left(k_{f} /[C]\right)$ in Arrheniusdiagrammen aufgeführt.

\subsubsection{Benzol}

Abbildung (3.16) zeigt in einem Arrheniusdiagramm die Temperaturabhängigkeit der Massenwachstumskonstante $k_{f}$ für drei verschiedene Kohlenstoffkonzentrationen. Mit zunehmender Temperatur und Kohlenstoffkonzentration nimmt die Massenwachstumskonstante stetig zu. Die präexponentiellen Faktoren nehmen stetig mit zunehmender Kohlenstoffkonzentration, von 1.3 auf 3.3 und $7.6 \times 10^{7} / \mathrm{s}$ zu und die scheinbare Aktivierungsenergie beträgt für alle drei Kohlenstoffkonzentrationen $145 \mathrm{~kJ} / \mathrm{mol}$. Unter Berücksichtigung des empirischen Gesetzes aus Gleichung (3.9) wird die Geschwindigkeitskonstante auf die eingesetzte Kohlenstoffkonzentration normiert und in Abbildung (3.17) aufgetragen. Innerhalb des Kohlenstoffdichtebereiches von 0,40 bis $4,0 \mathrm{~mol} / \mathrm{m}^{3}$ wird die Temperaturabhängigkeit von einer einzigen Geraden wiedergegeben. Nach der Normierung beträgt die scheinbare Aktivierungsenergie $205 \mathrm{~kJ} / \mathrm{mol}$. Der Druck wurde hier von 0,5 bis $11 \mathrm{MPa}$ variiert und kein Druckeinfluß festgestellt.

\subsubsection{Phenylacetylen}

In Abbildung (3.18) ist die normierte Massenwachstumskonstante für die Rußbildung aus $500 \mathrm{ppm}$ Phenylacetylen bei 5,7 MPa dargestellt. Im Gegensatz zu Benzol hat die Temperaturabhängigkeit der Massenwachstumskonstante bei $T_{\max }^{k_{f}}=1890 \mathrm{~K}$ ein ausgeprägtes Maximum. Bis zu dieser Temperatur sind die normierten Massenwachstumskonstanten etwa doppelt so groß wie in der Benzolpyrolyse. Sowohl der Bereich von $T_{\max }^{k_{f}}$ 


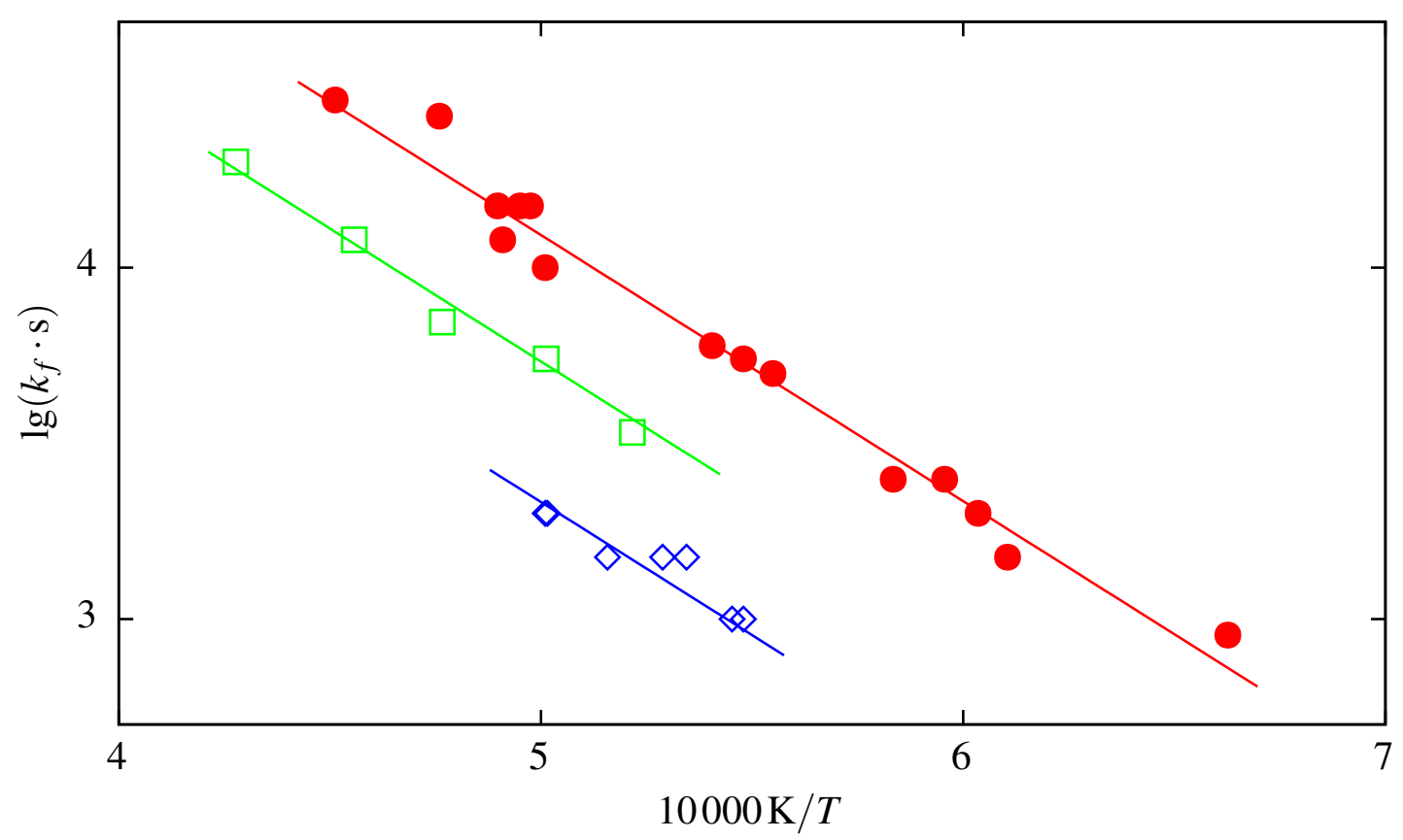

Abbildung 3.16: Temperaturabhängigkeit der Massenwachstumskonstante für drei verschiedene Benzolkonzentrationen und einem Druck von $5 \mathrm{MPa}$. Die Kohlenstoffkonzentrationen betragen: $\diamond: 0,4, \square: 1$ und $\bullet: 4 \mathrm{~mol} / \mathrm{m}^{3}$.

zu niedrigen Temperaturen als auch zu höheren Temperaturen kann mit je einer Geraden beschrieben werden. Die scheinbare Aktivierungsenergie beträgt im Niedertemperaturbereich $205 \mathrm{~kJ} / \mathrm{mol}$ und $-93 \mathrm{~kJ} / \mathrm{mol}$ im Hochtemperaturbereich.

\subsubsection{Acetylen}

Abbildung (3.19) zeigt die normierte Massenwachstumskonstante für die Rußbildung aus Acetylen. Die Kohlenstoffkonzentrationen wurde zwischen $([C]=0,9,1,7$ und $3,8 \mathrm{~mol} / \mathrm{m}^{3}$ ) variiert, der Druck zwischen 2,6 und 5,7 MPa. Hier ist das Maximum der Massenwachstumskonstante bei einer Temperatur von $T_{\max }^{k_{f}}=1960 \mathrm{~K}$ erreicht. Die scheinbare Aktivierungsenergie beträgt im Niedertemperaturbereich $100 \mathrm{~kJ} / \mathrm{mol}$ und $-93 \mathrm{~kJ} / \mathrm{mol}$ im Hochtemperaturbereich.

\subsubsection{Ethylen}

Abbildung (3.20) zeigt die normierte Massenwachstumskonstante für die Rußbildung aus Ethylen. Die Kohlenstoffkonzentration wurde von 3,9 bis $12 \mathrm{~mol} / \mathrm{m}^{3}$ variiert und der Druck zwischen 0,6 und $11 \mathrm{MPa}$. Bei einer Temperatur von $T_{\max }^{k_{f}}=1920 \mathrm{~K}$ hat die Massenwachstumskonstante ein ausgeprägtes Maximum. Die scheinbare Aktivierungsenergie beträgt im Niedertemperaturbereich $205 \mathrm{~kJ} / \mathrm{mol}$ und $-93 \mathrm{~kJ} / \mathrm{mol}$ im Hochtemperaturbereich. In Abbildung (3.21) sind die normierten Geschwindigkeitskonstanten aufgetragen, die hinter einfallenden Stoßwellen mit einem $3 \%$ Ethylen-Gemisch bei einem Druck von 0,53 MPa und einer Kohlenstoffkonzentration von 2,0 mol $/ \mathrm{m}^{3}$ gemessen wurden. Die Unterschiede der Arrhenius-Parameter, die aus Messungen hinter einfallenden und reflektierten Stoßwellen erhalten wurden, sind vernachlässigbar klein. 


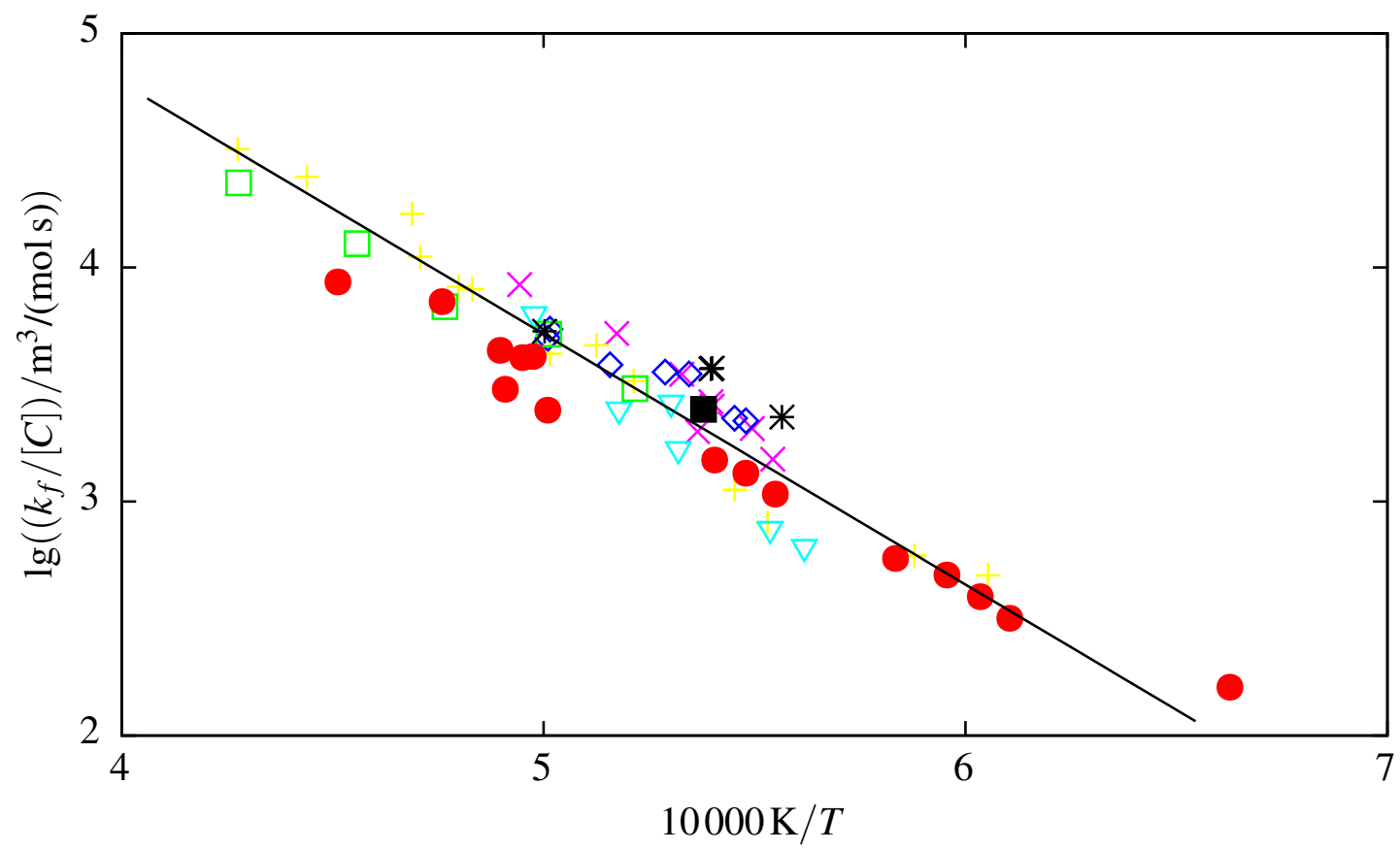

Abbildung 3.17: Temperaturabhängigkeit der auf die Kohlenstoffkonzentration normierten Massenwachstumskonstante für die Rußbildung in der Benzolpyrolyse. Die Drücke und Kohlenstoffkonzentration betragen: $P=0,5 \mathrm{MPa}, \times: 0,4 \mathrm{~mol} / \mathrm{m}^{3} . P=2,5 \mathrm{MPa}$, $+: 1,1 \mathrm{~mol} / \mathrm{m}^{3} \cdot P=5 \mathrm{MPa}, \diamond: 0,4, \nabla: 0,8, \square: 1,0$, und $\bullet: 4,0 \mathrm{~mol} / \mathrm{m}^{3} . P=11 \mathrm{MPa}, *: 0,4$ und $\mathbf{~}: 0,8 \mathrm{~mol} / \mathrm{m}^{3}$.

\subsubsection{Methan}

Zur Untersuchung der Temperaturabhängigkeit der normierten Rußmassenwachstumskonstante in der Methanpyrolyse wurde die Methankonzentration von $1,7 \mathrm{bis} 6,4 \mathrm{~mol} / \mathrm{m}^{3}$ variiert, und der Druck zwischen 2,5 und 5,5 MPa, siehe Abbildung (3.22). Bei einer Temperatur von $T_{\max }^{k_{f}}=2000 \mathrm{~K}$ hat die Massenwachstumskonstante ein Maximum. Die scheinbare Aktivierungsenergie beträgt im Niedertemperaturbereich $205 \mathrm{~kJ} / \mathrm{mol}$ und $-93 \mathrm{~kJ} / \mathrm{mol}$ im Hochtemperaturbereich.

\subsection{6 n-Hexan}

In Abbildung (3.23) sind die neu überarbeiteten Meßwerte der Massenwachstumskonstante für n-Hexan aus der Diplomarbeit von Tanke [19] aufgetragen. Im Unterschied zu dieser Arbeit wurden dort die Massenwachstumskonstanten aus Auftragungen entsprechend der Geradengleichung (3.3) bestimmt. Die neuen $k_{f}$-Werte sind im Mittel um 1/3 kleiner. Die Kohlenstoffdichte wurde konstant bei $5,3 \mathrm{~mol} / \mathrm{m}^{3}$ gehalten und der Druck von 2 bis $10 \mathrm{MPa}$ variiert. Bei einer Temperatur von $T_{\max }^{k_{f}}=1990 \mathrm{~K}$ hat die Massenwachstumskonstante ein Maximum. Die scheinbare Aktivierungsenergie beträgt im Niedertemperaturbereich $205 \mathrm{~kJ} / \mathrm{mol}$ und $-93 \mathrm{~kJ} / \mathrm{mol}$ im Hochtemperaturbereich. Eine Druckabhängigkeit wurde nicht gefunden werden. 


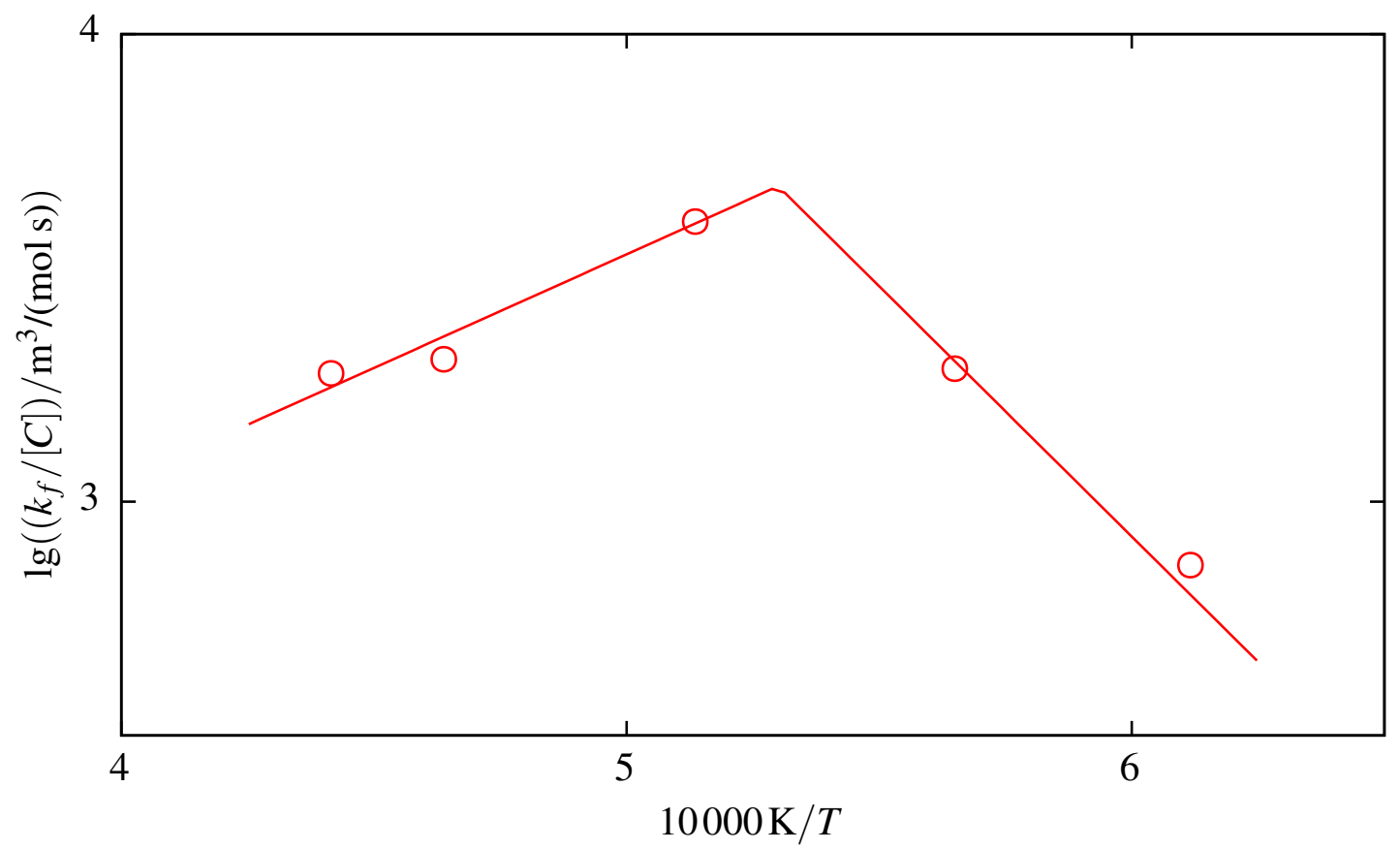

Abbildung 3.18: Temperaturabhängigkeit der auf die Kohlenstoffkonzentration normierten Massenwachstumskonstante für die Rußbildung in der Phenylacetylenpyrolyse. $[C]=1,4 \mathrm{~mol} / \mathrm{m}^{3}$ und $P=5,7 \mathrm{MPa}$.

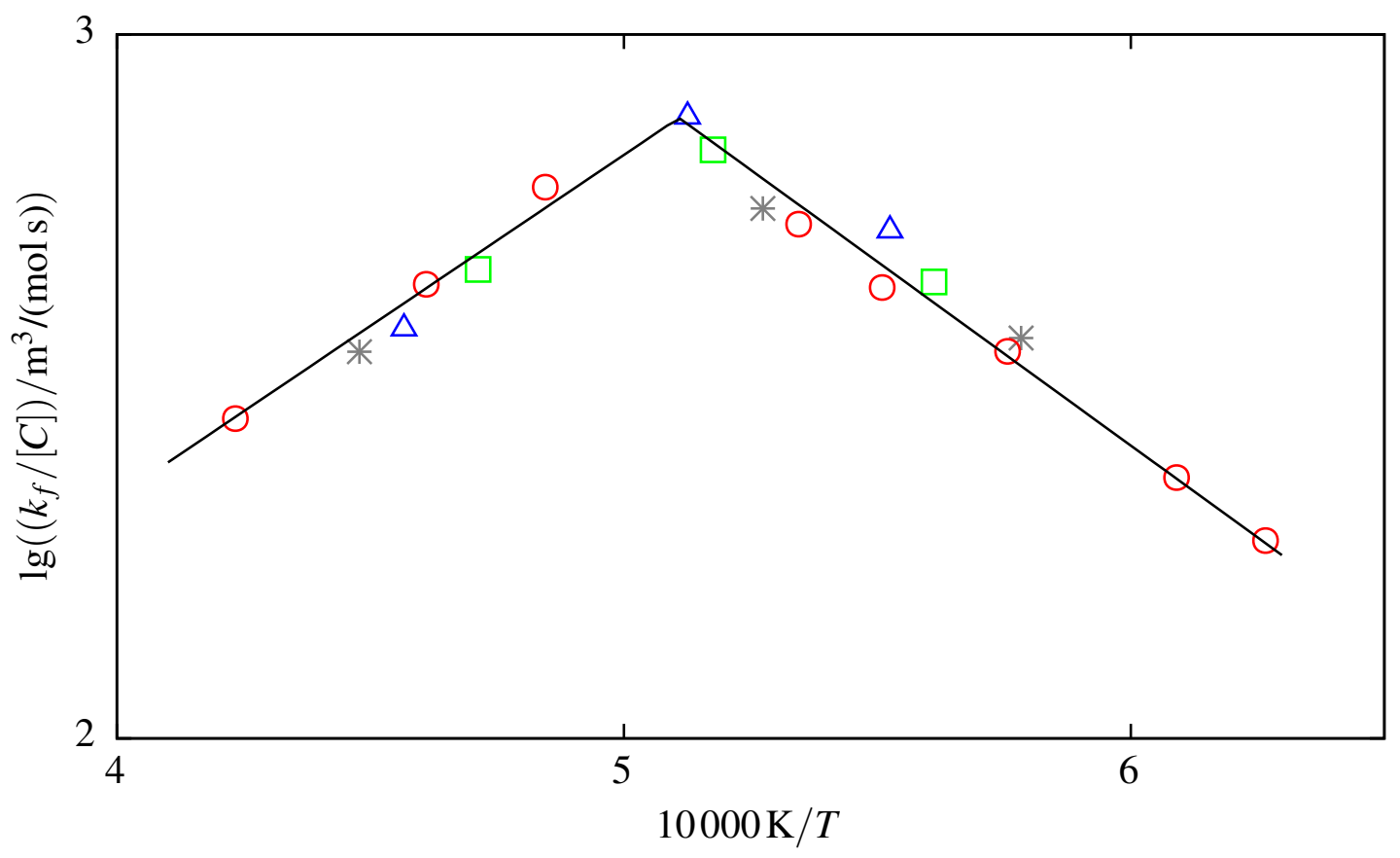

Abbildung 3.19: Temperaturabhängigkeit der auf die Kohlenstoffkonzentration normierten Massenwachstumskonstante für die Rußbildung in der Acetylenpyrolyse. Die angegebenen Konzentrationen beziehen sich auf die C-Atome. ०: $5,7 \mathrm{MPa}, 3,8 \mathrm{~mol} / \mathrm{m}^{3}$ $\square: 5,7 \mathrm{MPa}, 1,7 \mathrm{~mol} / \mathrm{m}^{3} \triangle: 5,7 \mathrm{MPa}, 0,9 \mathrm{~mol} / \mathrm{m}^{3}$ und $*: 2,6 \mathrm{MPa}, 1,7 \mathrm{~mol} / \mathrm{m}^{3}$. 


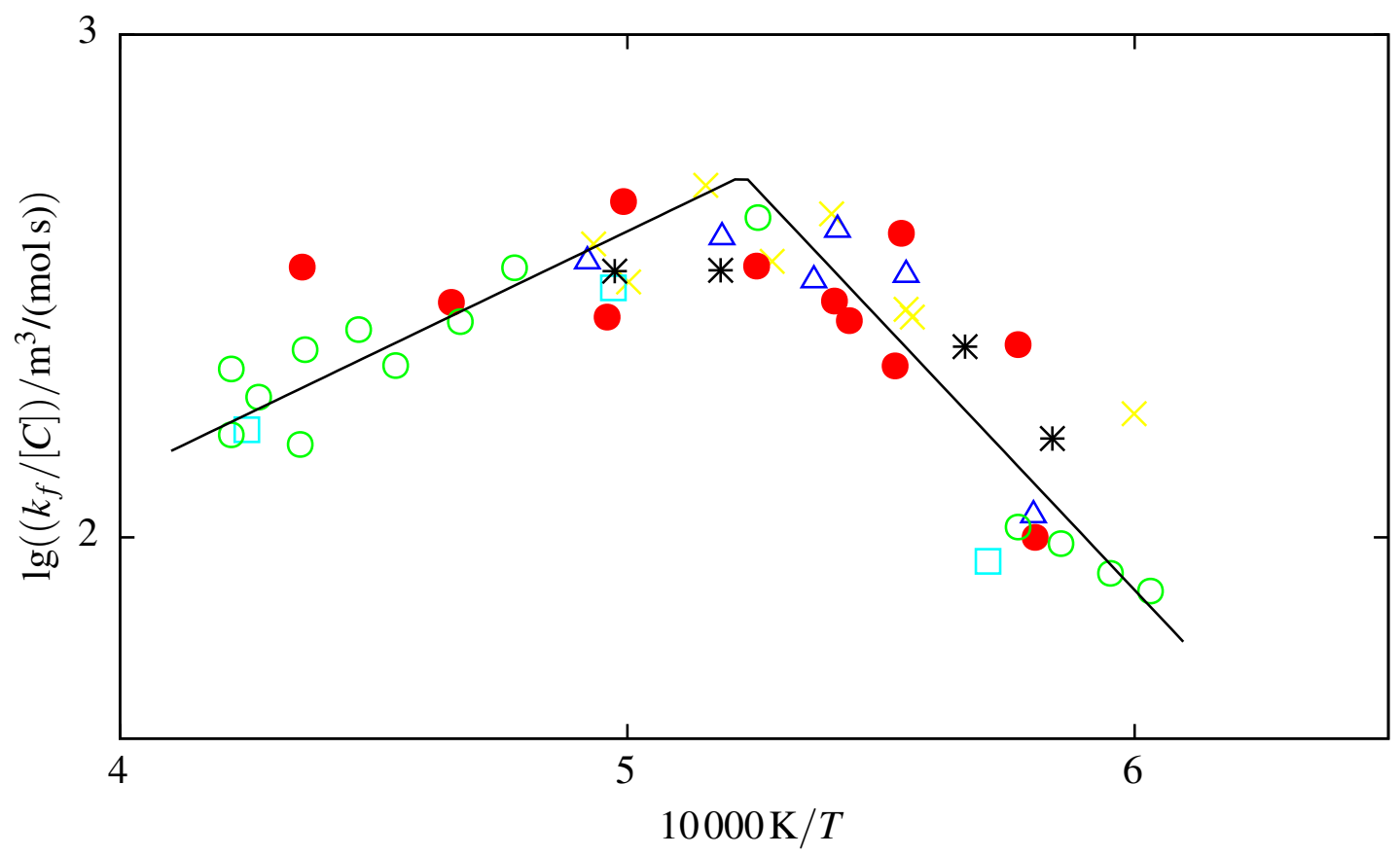

Abbildung 3.20: Temperaturabhängigkeit der auf die Kohlenstoffkonzentration normierten Massenwachstumskonstante für die Rußbildung in der Ethylenpyrolyse für verschiedene Kohlenstoffkonzentrationen und Drücke. Die angegebenen Konzentrationen beziehen sich auf die C-Atome. $\times: 0,6 \mathrm{MPa}, 3,9 \mathrm{~mol} / \mathrm{m}^{3} \triangle: 2,5 \mathrm{MPa}, 4,0 \mathrm{~mol} / \mathrm{m}^{3} \square: 2,5 \mathrm{MPa}$, $12 \mathrm{~mol} / \mathrm{m}^{3} \bullet: 5,0 \mathrm{MPa}, 4,2 \mathrm{~mol} / \mathrm{m}^{3}$ ॰: $5,0 \mathrm{MPa}, 7,0 \mathrm{~mol} / \mathrm{m}^{3}$ und $*: 11 \mathrm{MPa}, 4,2 \mathrm{~mol} / \mathrm{m}^{3}$.

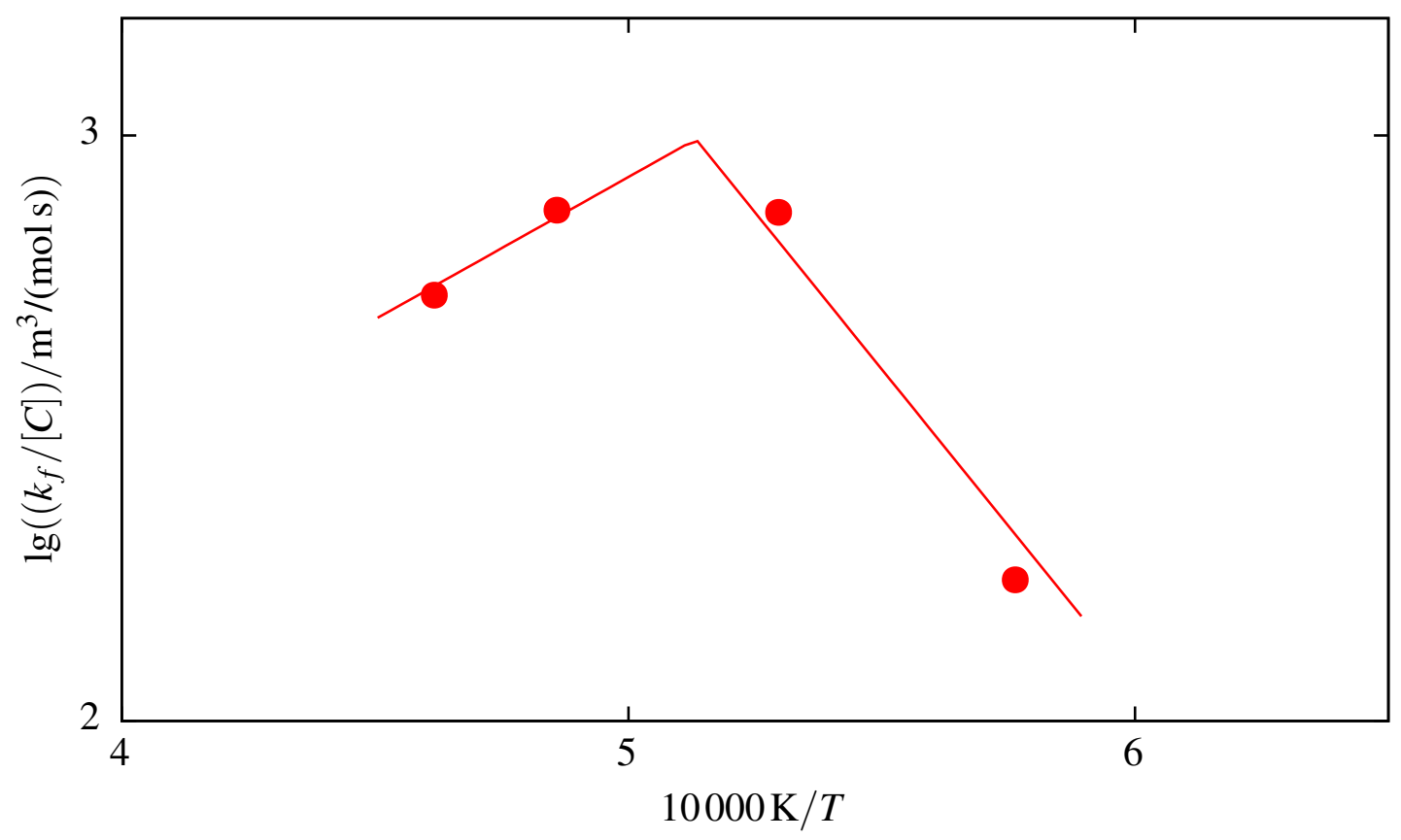

Abbildung 3.21: Temperaturabhängigkeit der normierten Massenwachstumskonstante für die Rußbildung in der Ethylenpyrolyse hinter einfallenden Stoßwellen. $P=0,53 \mathrm{MPa}$ und $[C]=2,0 \mathrm{~mol} / \mathrm{m}^{3}$. 


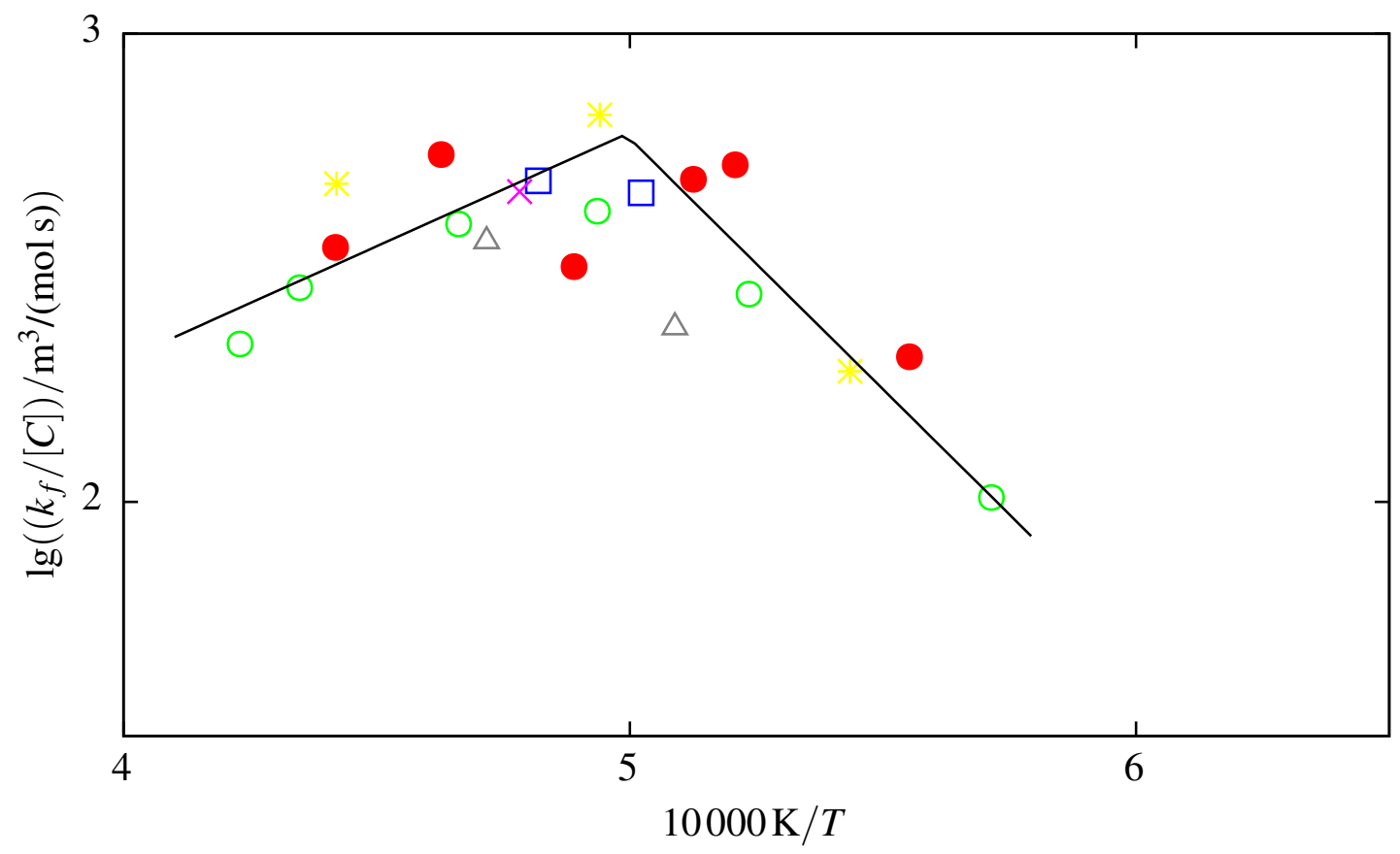

Abbildung 3.22: Temperaturabhängigkeit der auf die Kohlenstoffkonzentration normierten Massenwachstumskonstante für die Rußbildung in der Methanpyrolyse. $*:$ 2,5 MPa, $3,0 \mathrm{~mol} / \mathrm{m}^{3} \bullet: 5,5 \mathrm{MPa}, 3,4 \mathrm{~mol} / \mathrm{m}^{3} \times: 12 \mathrm{MPa}, 3,4 \mathrm{~mol} / \mathrm{m}^{3} \square: 5,5 \mathrm{MPa}, 1,7 \mathrm{~mol} / \mathrm{m}^{3}$ ○: $5,5 \mathrm{MPa}, 6,4 \mathrm{~mol} / \mathrm{m}^{3}$ und $\triangle: 24 \mathrm{MPa}, 28 \mathrm{~mol} / \mathrm{m}^{3}$.

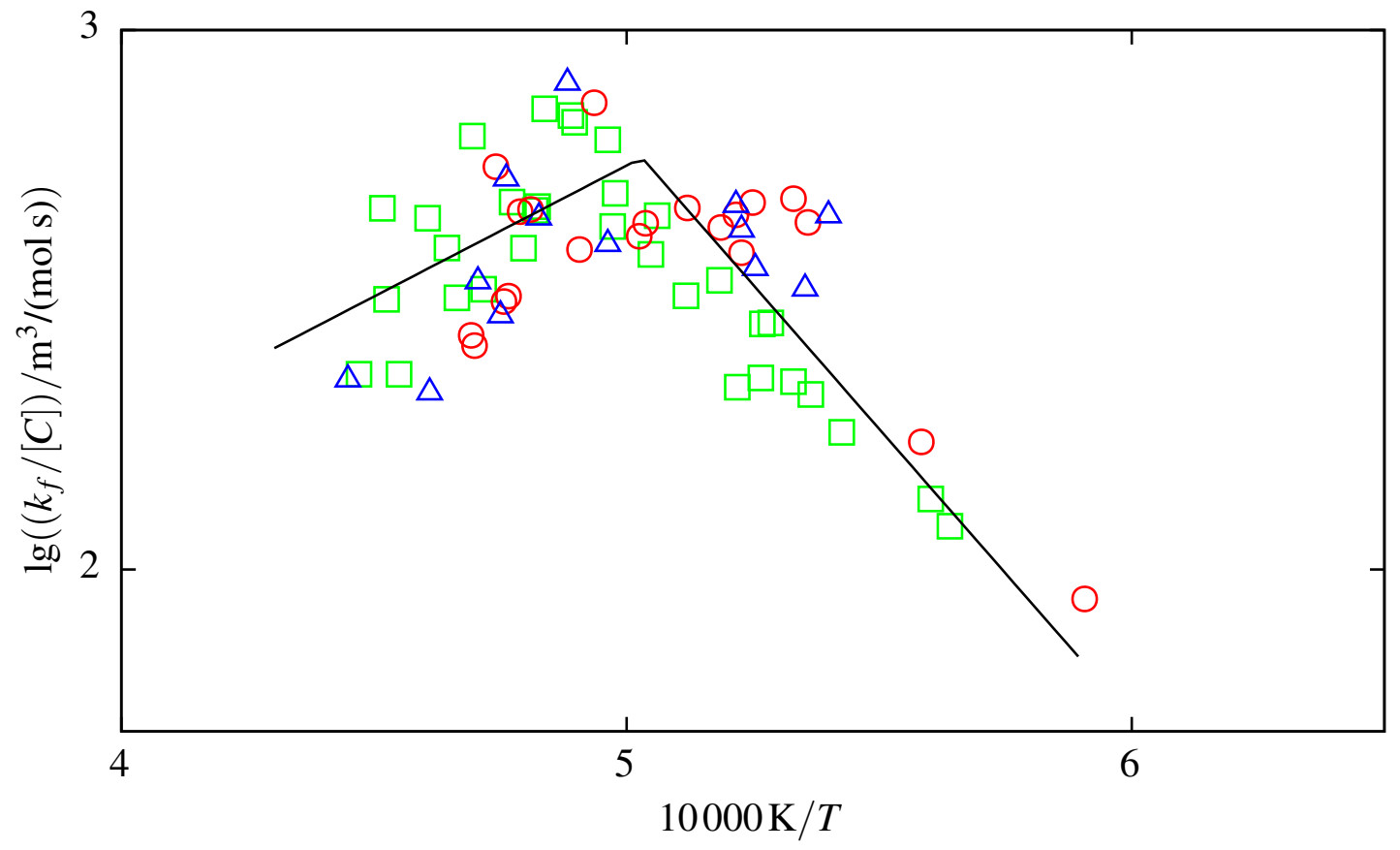

Abbildung 3.23: Temperaturabhängigkeit der auf die Kohlenstoffkonzentration normierten Massenwachstumskonstante für die Rußbildung in der Hexanpyrolyse. $[C]$ $=5,3 \mathrm{~mol} / \mathrm{m}^{3}, \square: 2,5, \circ: 6$ und $\triangle: 10 \mathrm{MPa}$. 


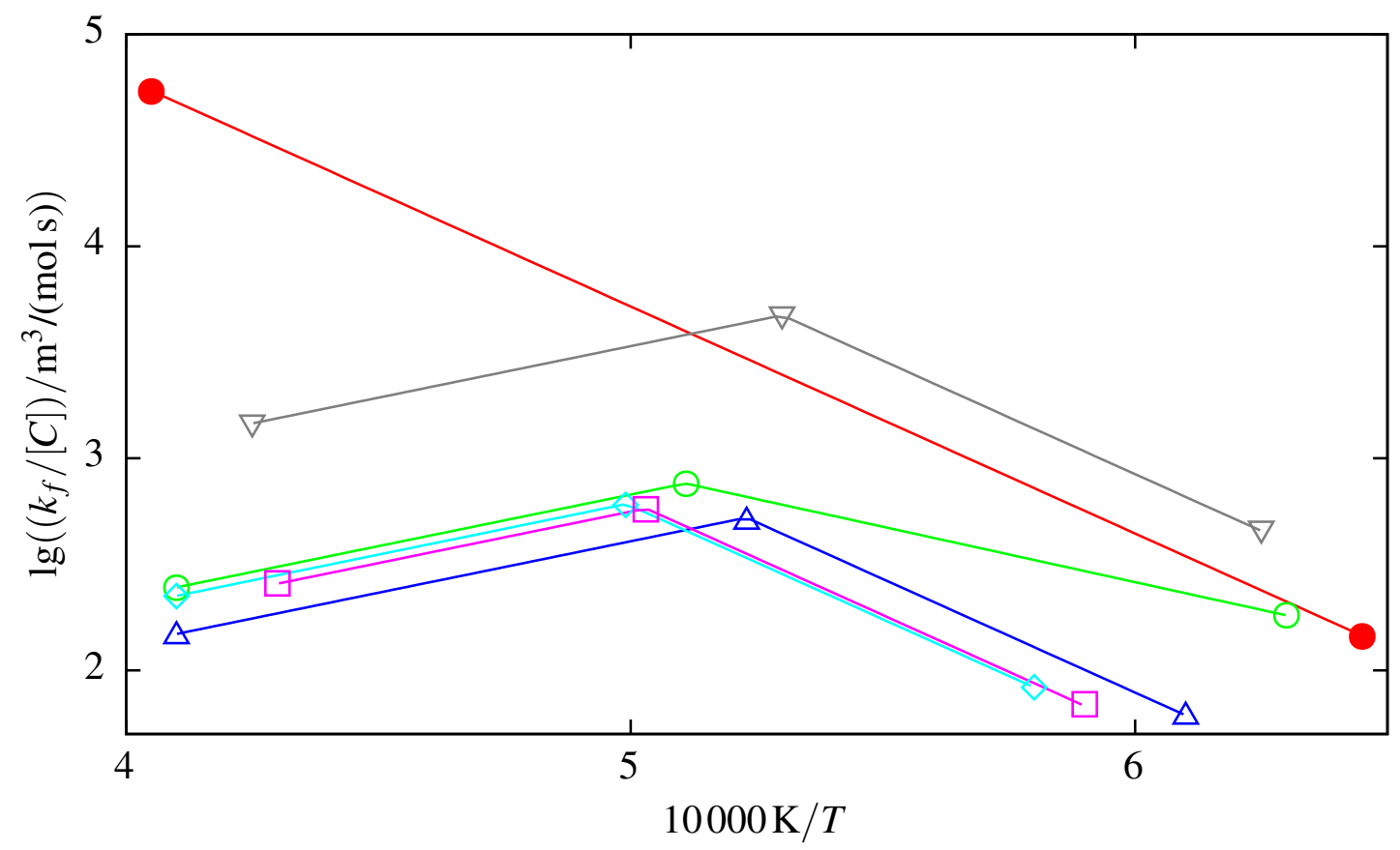

Abbildung 3.24: Vergleich der normierten Massenwachstumskonstante $\left(k_{f} /[C]\right)$ in Abhängigkeit der Temperatur für verschiedene Kohlenwasserstoffe. Die angegebenen Konzentrationen beziehen sich auf die C-Atome. $\bullet$ : Benzol 0,4 bis $4,0 \mathrm{~mol} / \mathrm{m}^{3} \nabla:$ Phenylacetylen $1,4 \mathrm{~mol} / \mathrm{m}^{3} \circ$ : Acetylen $0,9 \mathrm{bis} 3,8 \mathrm{~mol} / \mathrm{m}^{3} \triangle$ : Ethylen 3,9 bis $12 \mathrm{~mol} / \mathrm{m}^{3} \diamond:$ Methan 1,7 bis $6,4 \mathrm{~mol} / \mathrm{m}^{3}$ und $\square$ : n-Hexan 5,3 bis $35 \mathrm{~mol} / \mathrm{m}^{3}$.

\subsubsection{Vergleich der Kohlenwasserstoffe}

Zusammenfassend ist die Abhängigkeit der normierten Massenwachstumskonstante $\left(k_{f} /[C]\right)$ von der Temperatur und vom eingesetzten Kohlenwasserstoff in Abbildung (3.24) und in Tabelle (3.2) dargestellt. Abgesehen von Benzol haben die normierten Massenwachstumskonstanten der untersuchten Kohlenwasserstoffe bei (1950 \pm 50$) \mathrm{K}$ ein Maximum. Die scheinbare Aktivierungsenergie ist im Hochtemperaturbereich für diese Kohlenwasserstoffe konstant $-93 \mathrm{~kJ} / \mathrm{mol}$. Im Niedertemperaturbereich beträgt die scheinbare Aktivierungsenergie $205 \mathrm{~kJ} / \mathrm{mol}$. Nur für Acetylen wurde eine deutlich kleinere scheinbare Aktivierungsenergie gefunden. Das Rußmassenwachstum der Aromaten ist über den ganzen Temperaturbereich mindestens eine Größenordnung schneller als das Rußmassenwachstum der Aliphaten. Die Unterschiede zwischen den Aliphaten sind kleiner als die experimentellen Fehler. Besonders auffällig ist die Geschwindigkeit des Rußmassenwachstums in der Acetylenpyrolyse. Acetylen verhält sich bei niedrigen Temperaturen wie die Aromaten und mit zunehmender Temperatur ebenso wie die anderen Aliphaten. Dieser Übergang der Reaktionsgeschwindigkeit findet im Temperaturbereich zwischen 1600 und $2000 \mathrm{~K}$ statt.

\subsection{Rußausbeute}

Das Rußmassenwachstum ist nach hinreichend langer Reaktionszeit beendet. In dem charakteristischen Rußausbeute-Zeitprofil in Abbildung (3.2) hat sich bei ca. 1,5 ms eine konstant bleibende Rußmenge $\left(R A_{\infty}\right)$ gebildet. Im keinem Fall wurde ein anschließen- 


\begin{tabular}{|c|c|c|c|c|c|}
\hline Edukt & $T / \mathrm{K}$ & {$[C] / \frac{\mathrm{mol}}{\mathrm{m}^{3}}$} & $T_{\max }^{k_{f}} / \mathrm{K}$ & $\lg (A / \mathrm{s})$ & $E_{k f} / \frac{\mathrm{kJ}}{\mathrm{mol}}$ \\
\hline Benzol & 1600 bis 2400 & 0,4 bis 4,0 & - & 9,07 & 205 \\
\hline Phenylacetylen & 1600 bis 2300 & 1,4 & 1890 & $9,35 \quad 1,10$ & $205-93$ \\
\hline Acetylen & 1600 bis 2400 & 0,9 bis 3,8 & 1960 & $5,55 \quad 0,40$ & $100-93$ \\
\hline Ethylen & 1650 bis 2400 & 3,9 bis 12 & 1910 & $8,320,18$ & $205-93$ \\
\hline Methan & 1700 bis 2400 & 1,7 bis 6,4 & 2000 & $8,130,36$ & $205-93$ \\
\hline n-Hexan & 1700 bis 2250 & 5,3 & 1990 & $8,15 \quad 0,32$ & $205-93$ \\
\hline
\end{tabular}

Tabelle 3.2: Die Maximumtemperatur $T_{\max }^{k_{f}}$ und die zwei Arrhenius-Parameter $A, E_{k f}$ zur Berechnung der normierten Massenwachstumskonstante $\left(k_{f} /[C]\right)$ für verschiedene Kohlenwasserstoffe. In den Spalten fünf und sechs gilt der linke Wert für den Nieder- und der rechte Wert für den Hochtemperaturbereich.

der Abbau des Rußes beobachtet. Über die Temperaturabhängigkeit der Rußausbeute in der Kohlenwasserstoffpyrolyse wurde erstmals von Graham [1] berichtet. Bei einer Temperatur von $1800 \mathrm{~K}$ hat die Rußausbeute ein ausgeprägtes Maximum. Ein ähnliches Verhalten der Rußausbeute wurde von Frenklach et al [2] für die Toluolpyrolyse und von Hwang et al [5] für die Hexanpyrolyse bestätigt. Zu höheren und niedrigeren Temperaturen nimmt die Rußausbeute bis unter die Nachweisgrenze ab. Die Auftragungen haben die Form von unsymmetrischen „Glockenkurven“. Mit einem einfachen Drei-SpeziesModell, indem angenommen wird, daß sich die Spezies in gekoppelten Gleichgewichten befinden, wurde die Temperaturabhängigkeit der Rußausbeute quantitativ beschrieben.

$$
\text { Edukt } \underset{k_{21}}{\stackrel{k_{12}}{\rightleftharpoons}} \text { Zwischenprodukte } \underset{k_{32}}{\stackrel{k_{23}}{\rightleftharpoons}} \text { Ruß }
$$

In der Diskussion wird das Modell eingehend erläutert. Die in den folgenden Abbildungen dargestellten Meßwerte der Rußausbeute konnten nach diesem Modell mit den durchgezogenen Kurven gut angepaßt werden. Die Höhe der Rußausbeute war unabhängig von den verwendeten Lichtwellenlängen, siehe Abbildung (3.3). Da der Absorptionskoeffizient $\alpha$ des Rußes im Bereich des sichtbaren und nahen infraroten Licht mit zunehmender Wellenlänge abnimmt, konnte die Laser-Diode $(\lambda=1064 \mathrm{~nm})$ in Experimenten mit hohen Rußausbeuten besonders vorteilhaft eingesetzt werden.

\subsubsection{Benzol}

In der Benzolpyrolyse wurden die Rußausbeuten in Abhängigkeit der Temperatur, der Kohlenstoffdichte und des Druckes bestimmt. Abbildung (3.25) zeigt die Temperaturund Kohlenstoffkonzentrationsabhängigkeit im Bereich von 1500 bis $2500 \mathrm{~K}$ und von 0,4 bis $4 \mathrm{~mol} / \mathrm{m}^{3}$ bei einem konstanten Druck von $5 \mathrm{MPa}$. Die maximale Rußausbeute wurde für jede untersuchte Kohlenstoffdichte bei einer Temperatur $\left(T_{\max }^{R A}\right)$ von $(1800 \pm 20) \mathrm{K}$ erreicht. Bei dieser Temperatur wurden Rußausbeuten bis zu $85 \%$ gemessen. Die Abnahme der Rußausbeute verläuft bei niedrigen Temperaturen steiler als bei höheren Temperaturen. Mit abnehmender Temperatur verlängerten sich zudem die Induktionszeiten und verkleinerten sich die Massenwachstumskonstanten. Diese Effekte waren so ausgeprägt, daß innerhalb der Meßzeit und unterhalb von $1700 \mathrm{~K}$ nur bei hohen Kohlenstoffkonzentrationen Ruß nachgewiesen wurde. Mit zunehmender Kohlenstoffkonzentration nahmen 


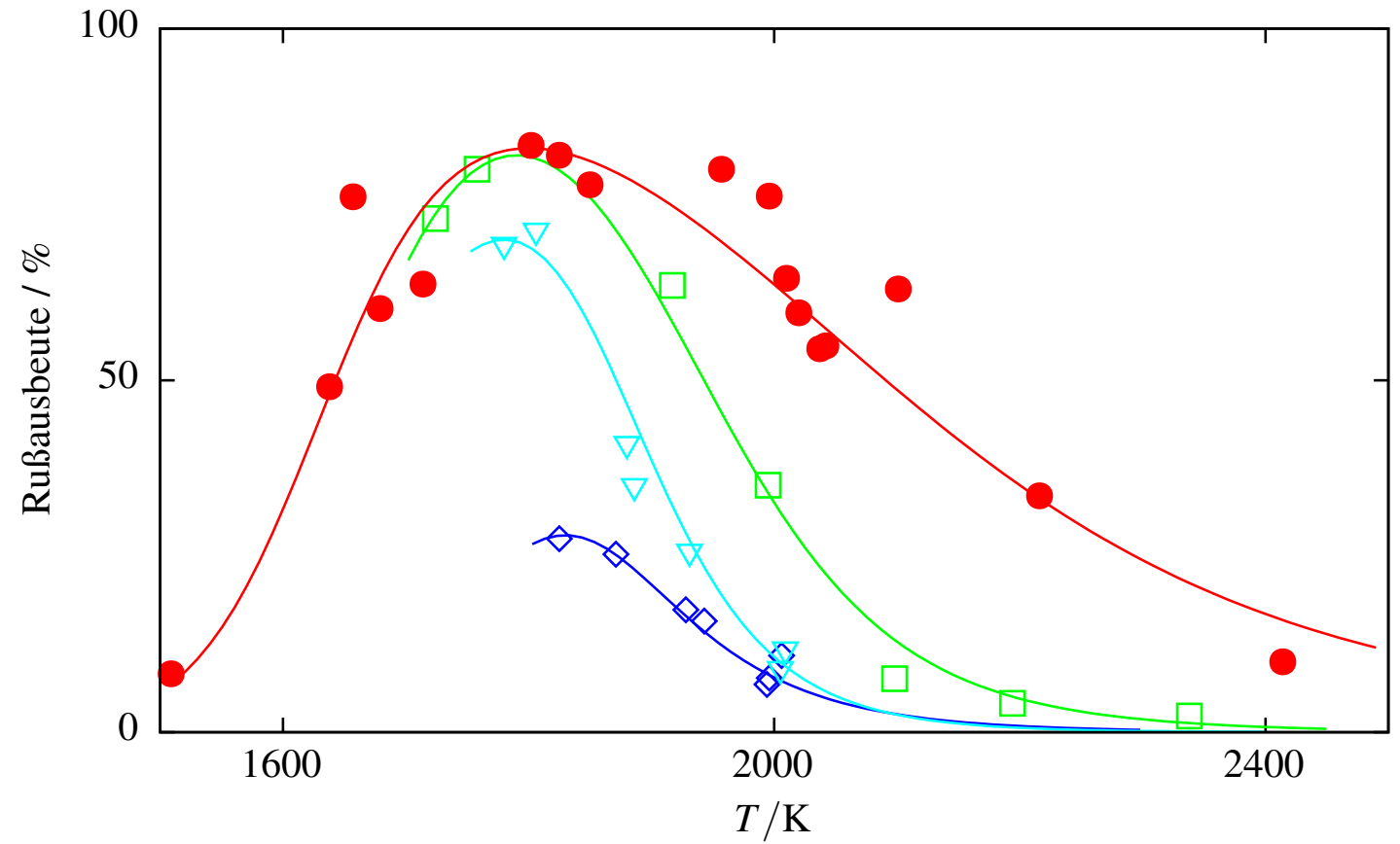

Abbildung 3.25: Temperaturabhängigkeit der Rußausbeute für vier verschiedene Benzolkonzentrationen bei einem konstanten Druck von $5 \mathrm{MPa}$. Die Kohlenstoffkonzentrationen betragen: $\diamond: 0,4, \nabla: 0,8, \square: 1$ und $\bullet: 4 \mathrm{~mol} / \mathrm{m}^{3}$.

die Rußausbeuten stark zu. Eine Verdoppelung der Kohlenstoffkonzentration von 0,4 auf $0,8 \mathrm{~mol} / \mathrm{m}^{3}$ erhöhte die Rußausbeute bei $1800 \mathrm{~K}$ von $30 \%$ auf $70 \%$.

Die Druckabhängigkeit der Rußausbeute wurde bei einer konstanten Kohlenstoffdichte von $0,4 \mathrm{~mol} / \mathrm{m}^{3}$ untersucht, siehe Abbildung (3.26). Bei der Maximumtemperatur und $5,6 \mathrm{MPa}$ beträgt die Rußausbeute $30 \%$. Mit abnehmendem oder zunehmendem Druck auf 0,56 bzw. 10,6 MPa nimmt die Rußausbeute um mehr als Faktor zwei zu.

\subsubsection{Phenylacetylen}

In Abbildung (3.27) ist die Temperaturabhängigkeit der Rußausbeute für die Phenylacetylenpyrolyse bei einer mittleren Kohlenstoffdichte von $1,4 \mathrm{~mol} / \mathrm{m}^{3}$ dargestellt. Man erhält, unter sonst gleichen Bedingungen, die gleiche Rußausbeute wie in der Benzolpyrolyse.

\subsubsection{Acetylen}

Die Rußausbeute in der Acetylenpyrolyse wurde bei drei Kohlenstoffkonzentrationen $\left(0,9,1,7\right.$ und $\left.3,8 \mathrm{~mol} / \mathrm{m}^{3}\right)$ und zwei Drücken $(2,6$ und 5,7 MPa) untersucht. In Abbildung (3.28) sind die Ergebnisse aufgetragen. Die Rußausbeuten und die Abhängigkeit von der Kohlenstoffdichte sind denen der Benzolpyrolyse unter sonst gleichen Bedingungen sehr ähnlich. Nur bei niedrigen Temperaturen nimmt die Rußausbeute noch stärker ab als in der Benzolpyrolyse. Ein Druckeinfluß auf die Rußausbeute wurde nicht festgestellt. 


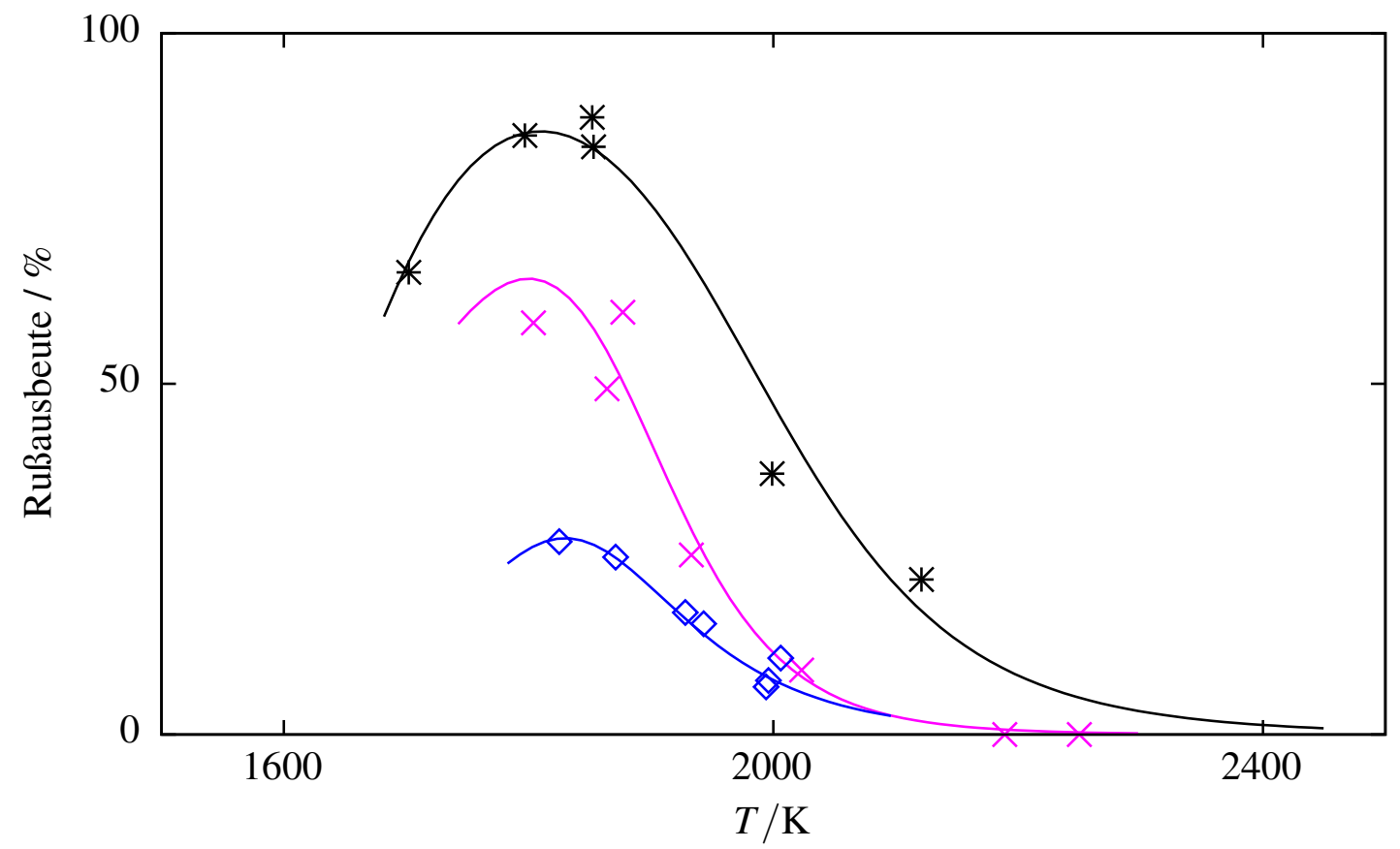

Abbildung 3.26: Temperaturabhängigkeit der Rußausbeute in der Benzolpyrolyse für drei verschiedene Drücke $(\times: 0,55, \diamond: 5,4$ und $*: 10,6 \mathrm{MPa})$ und konstanter Kohlenstoffkonzentration von $[C]=0,4 \mathrm{~mol} / \mathrm{m}^{3}$.

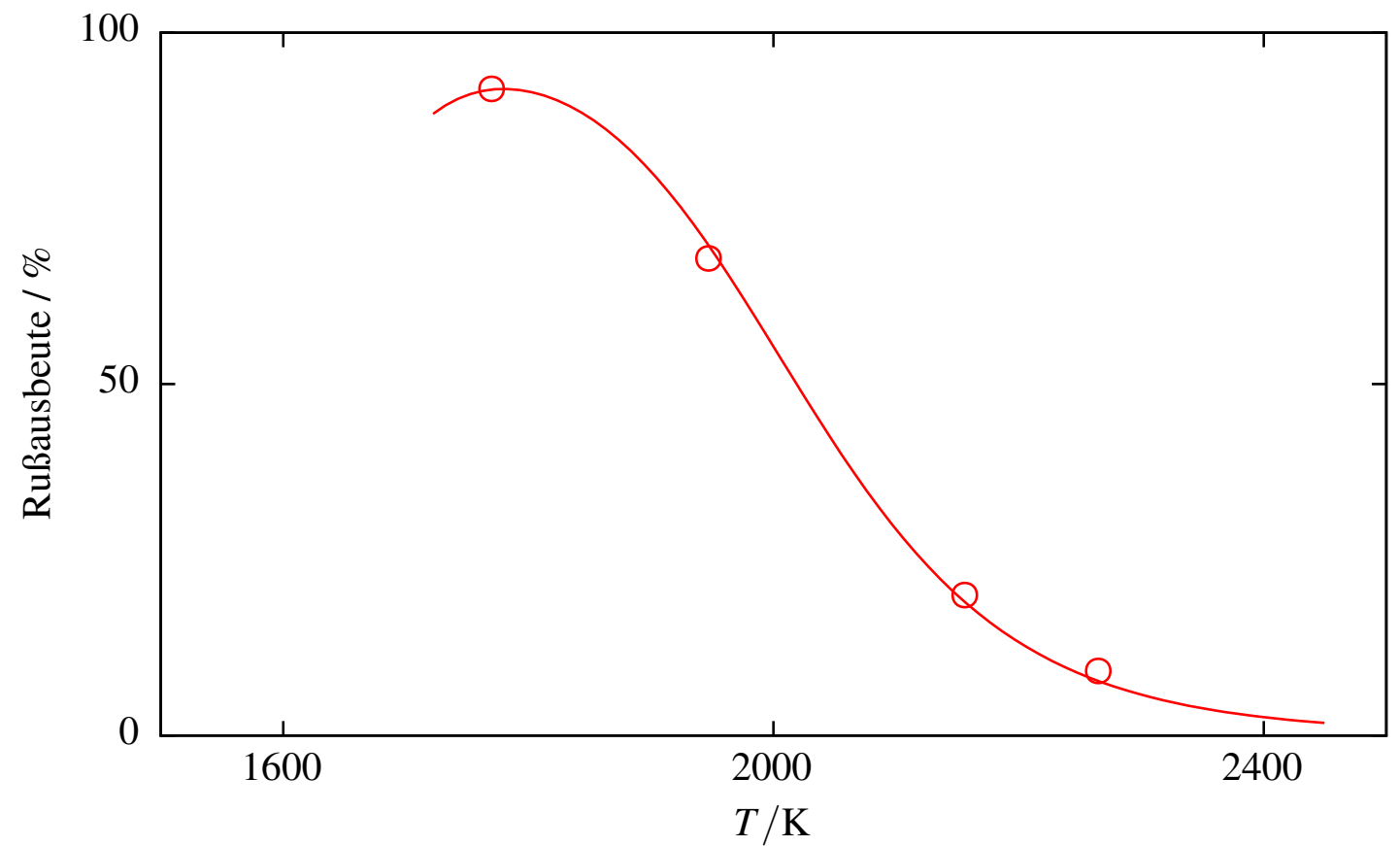

Abbildung 3.27: Temperaturabhängigkeit der Rußausbeute von Phenylacetylen bei einer Kohlenstoffkonzentration von $1,4 \mathrm{~mol} / \mathrm{m}^{3}$ und einem Druck von 5,7 MPa. 


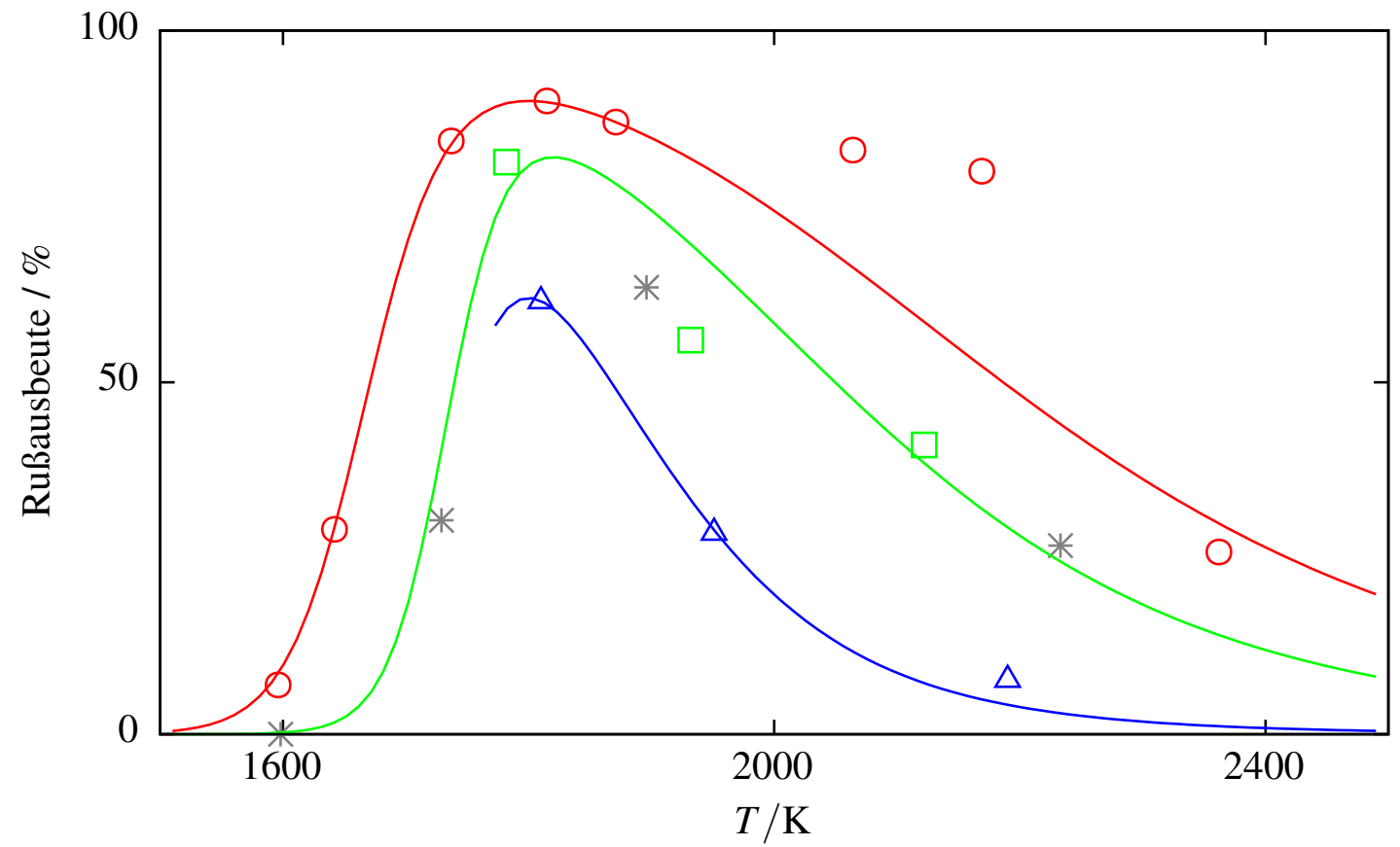

Abbildung 3.28: Temperaturabhängigkeit der Rußausbeute für drei verschiedene Acetylenkonzentrationen und zwei unterschiedlichen Drücken: o: $5,7 \mathrm{MPa}, 3,8 \mathrm{~mol} / \mathrm{m}^{3}$ $\square: 5,7 \mathrm{MPa}, 1,7 \mathrm{~mol} / \mathrm{m}^{3} \triangle: 5,7 \mathrm{MPa}, 0,9 \mathrm{~mol} / \mathrm{m}^{3}$ und $*: 2,6 \mathrm{MPa}, 1,7 \mathrm{~mol} / \mathrm{m}^{3}$. Die angegebenen Konzentrationen beziehen sich auf die C-Atome. Die Meßwerte bei unterschiedlichem Druck aber gleicher Kohlenstoffkonzentration ( $\square$ und $*$ ) wurden mit einer einzigen Kurve angepaßt.

\subsubsection{Ethylen}

Die Rußausbeuten in der Ethylenpyrolyse sind von der Temperatur, der Kohlenstoffdichte und dem Druck abhängig, siehe die Abbildungen (3.29) und (3.30). Die Kohlenstoffkonzentration wurde von 4,0 bis $7,4 \mathrm{~mol} / \mathrm{m}^{3}$, der Druck von 2,5 bis $11 \mathrm{MPa}$ variiert. Die Maximumtemperatur beträgt unter allen Bedingungen $(1850 \pm 20) \mathrm{K}$. Sowohl mit zunehmendem Druck als auch mit zunehmender Ethylendichte nehmen die Rußausbeuten stetig zu. Diese Zunahme der Rußausbeuten ist oberhalb der Maximumtemperatur größer als unterhalb der Maximumtemperatur. Im Vergleich zu den Aromaten und Acetylen sind die Rußausbeuten in der Ethylenpyrolyse deutlich niedriger. Nur im Bereich der Maximumtemperatur und hinreichend hohem Druck bzw. Ethylendichte sind die Rußausbeuten vergleichbar hoch. Zum Beispiel ist bei $2000 \mathrm{~K}, 5 \mathrm{MPa}$ und einer Kohlenstoffdichte von $4,0 \mathrm{~mol} / \mathrm{m}^{3}$ die Rußausbeute in der Ethylenpyrolyse um ca. $2 / 3$ kleiner als in der Acetylen- und Benzolpyrolyse.

\subsubsection{Methan}

Abbildung (3.31) zeigt die Rußausbeuten in der Methanpyrolyse für den Bereich von 1,7 bis $6,4 \mathrm{~mol} / \mathrm{m}^{3}$ und von 2,5 bis $12 \mathrm{MPa}$. Die mittlere Kurve ist den Rußausbeuten aus Messungen bei konstanter Kohlenstoffkonzentration $\left(3,4 \mathrm{~mol} / \mathrm{m}^{3}\right)$ und unterschiedlichen Drücken angepaßt. Die Maximumtemperatur beträgt $(1960 \pm 20)$ K. Bei dieser Temperatur beträgt die Rußausbeute $50 \%$ und ist somit ca. 1/3 kleiner als in der Benzol- und Acetylenpyrolyse. Der Druck hatte keinen Einfluß auf die Rußausbeute. 


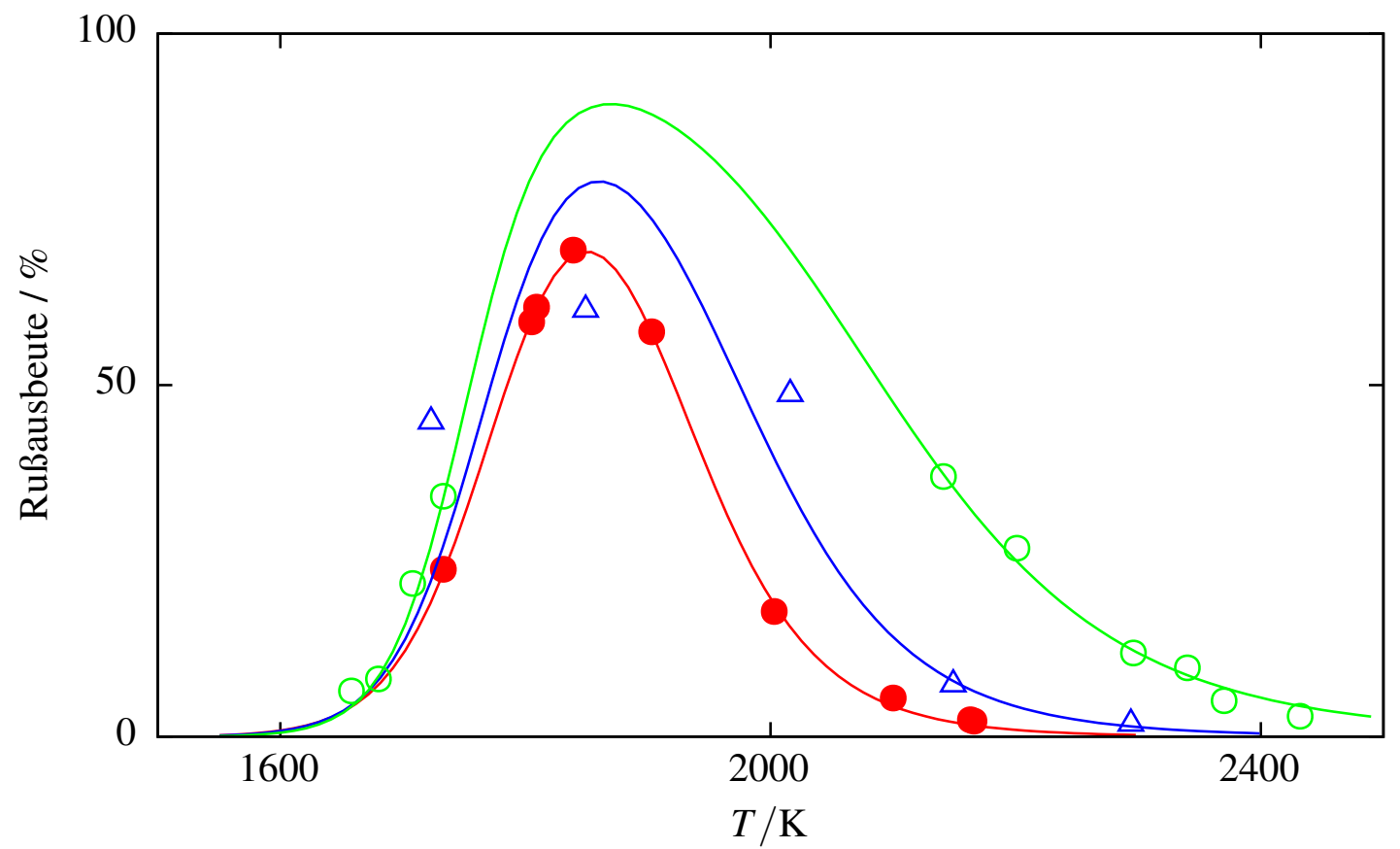

Abbildung 3.29: Temperaturabhängigkeit der Rußausbeute für verschiedene Ethylenkonzentrationen bei $P=5 \mathrm{MPa}$. Die Kohlenstoffkonzentrationen betragen: $\bullet: 4,0, \triangle: 4,7$ und ०: $7,4 \mathrm{~mol} / \mathrm{m}^{3}$.

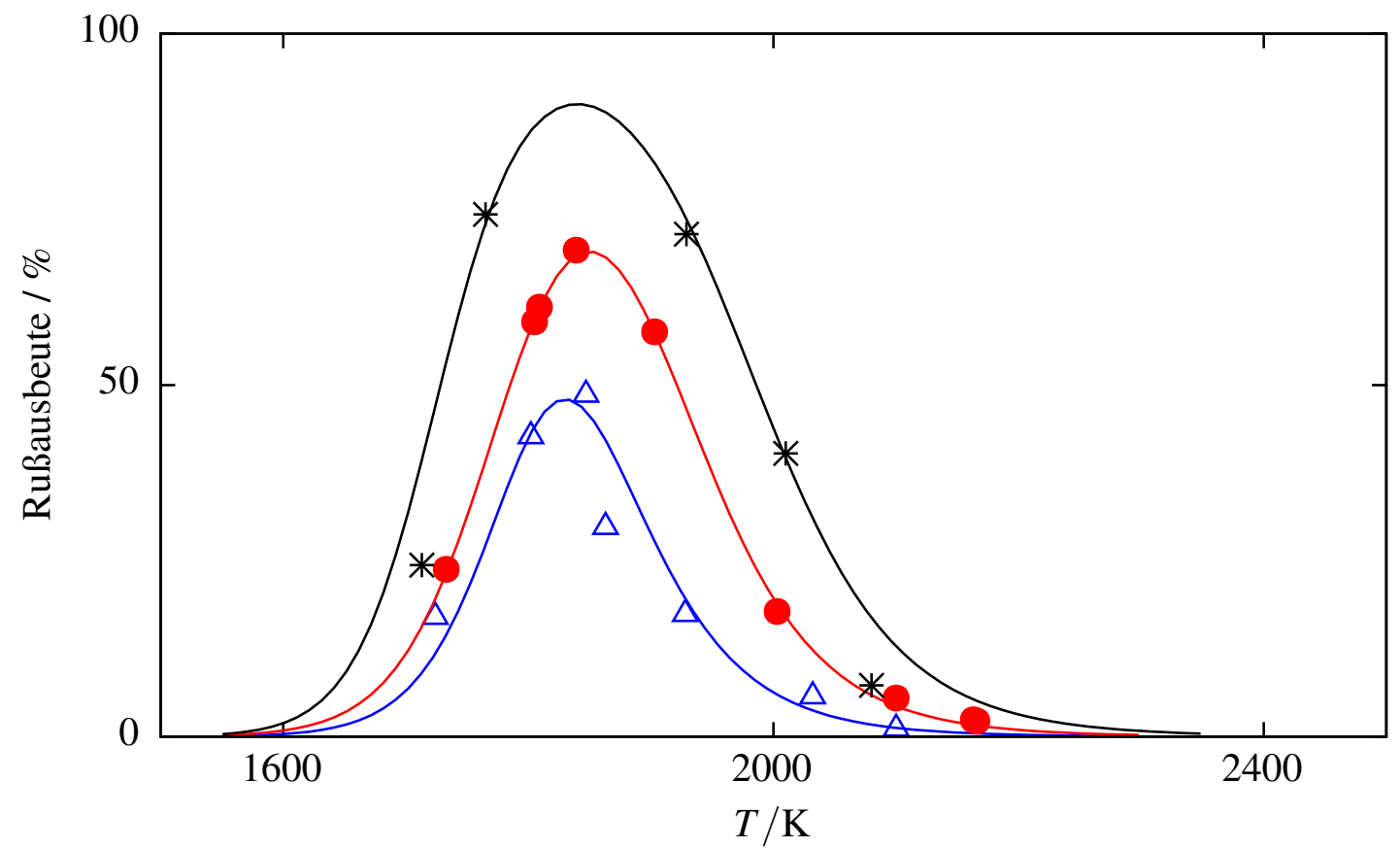

Abbildung 3.30: Temperaturabhängigkeit der Rußausbeute in der Ethylenpyrolyse für drei verschiedene Drücke $(\triangle: 2,5, \bullet: 5,0$ und $*: 11 \mathrm{MPa})$ und konstanter Kohlenstoffkonzentration $\left([C]=4 \mathrm{~mol} / \mathrm{m}^{3}\right)$. 


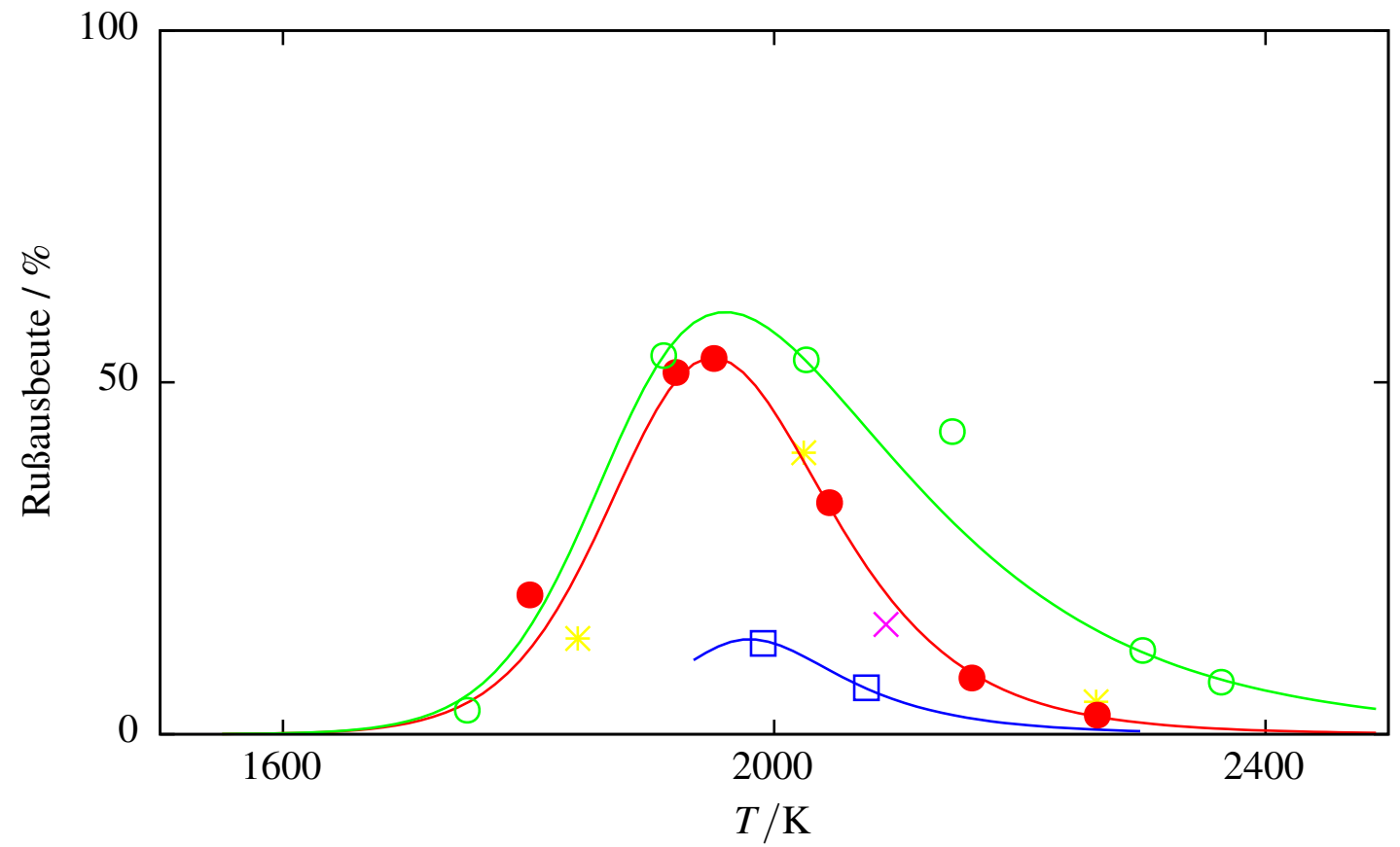

Abbildung 3.31: Temperaturabhängigkeit der Rußausbeute für verschiedene Methankonzentrationen und Drücke. $*: 2,5 \mathrm{MPa}, 3,0 \mathrm{~mol} / \mathrm{m}^{3} \bullet: 5,5 \mathrm{MPa}, 3,4 \mathrm{~mol} / \mathrm{m}^{3} \times: 12 \mathrm{MPa}$, $3,4 \mathrm{~mol} / \mathrm{m}^{3} \square: 5,5 \mathrm{MPa}, 1,7 \mathrm{~mol} / \mathrm{m}^{3}$ und $\circ: 5,5 \mathrm{MPa}, 6,4 \mathrm{~mol} / \mathrm{m}^{3}$.

\subsection{6 n-Hexan}

In Abbildung (3.32) sind die neu überarbeiteten Meßwerte der Rußausbeute für n-Hexan aus der Diplomarbeit von Tanke [19] aufgetragen. Im Unterschied zu dieser Arbeit wurden dort die Rußausbeuten aus Auftragungen entsprechend der Geradengleichung (3.3) bestimmt. Die neu gefitteten Werte sind im Mittel um 1/3 höher. Die Kohlenstoffdichte wurde konstant auf 5,3 mol $/ \mathrm{m}^{3}$ gehalten, der Druck von 2 bis $10 \mathrm{MPa}$ variiert. Ein Druckeinfluß wurde nicht festgestellt. Bei der Maximumtemperatur von $T_{\max }^{R A}=1950 \mathrm{~K}$ hat die Rußausbeute eine Höhe von $40 \%$. Das ist im Vergleich zu allen anderen untersuchten Kohlenwasserstoffen die geringste Rußausbeute.

\subsubsection{Vergleich der Kohlenwasserstoffe}

Die untersuchten Aromaten und Acetylen ergeben in der Pyrolyse ähnlich hohe Rußausbeuten. Die gemeinsame Maximumtemperatur liegt bei ca. $1800 \mathrm{~K}$ und die Rußausbeuten sind schon für relativ niedrige Kohlenstoffkonzentrationen hoch. Ethylen hat eine $50 \mathrm{~K}$, die Alkane haben eine $150 \mathrm{~K}$ höhere Maximumtemperatur. In der Diskussion wird ein Modell vorgeschlagen, indem mit nur vier Parameter die Temperaturabhängigkeit Rußausbeute beschrieben wird. Diese Parameter, mit denen nach Gleichung (4.7) die Rußausbeuten für alle untersuchten Kohlenwasserstoffe berechnet werden können, sind in Tabelle (3.3) angegeben. Die Reaktionsenthalpien $\Delta_{R} H_{12}$ sind relativ groß und betragen im Mittel $790 \mathrm{~kJ} / \mathrm{mol}$ für die Aromaten und $1080 \mathrm{~kJ} / \mathrm{mol}$ für die Nicht-Aromaten. Die Reaktionsenthalpie $\Delta_{R} H 23$ ist stets negativ, vom eingesetzten Kohlenwasserstoff wenig abhängig und nimmt mit zunehmender Kohlenstoffkonzentration zu. 


\begin{tabular}{lccccccc}
\hline & $T$ & {$[C]$} & $P$ & $T_{\max }^{R A}$ & $R A_{\max }$ & $\Delta_{R} H_{12}$ & $\Delta_{R} H_{23}$ \\
Edukt & {$[\mathrm{K}]$} & {$\left[\frac{\mathrm{mol}}{\mathrm{m}^{3}}\right]$} & {$[\mathrm{MPa}]$} & {$[\mathrm{K}]$} & {$[\%]$} & {$[R \cdot 1000 \mathrm{~K}]$} \\
\hline Benzol & 1800 bis 2050 & 0,4 & 0,56 & 1800 & 65 & 100 & -68 \\
Benzol & 1800 bis 2000 & 0,4 & 5,5 & 1825 & 28 & 90 & -50 \\
Benzol & 1700 bis 2150 & 0,4 & 10,6 & 1810 & 86 & 100 & -50 \\
Benzol & 1750 bis 2000 & 0,8 & 5,5 & 1780 & 70 & 100 & -65 \\
Benzol & 1700 bis 2350 & 1 & 5,5 & 1790 & 82 & 100 & -50 \\
Benzol & 1500 bis 2500 & 4 & 5,5 & 1800 & 83 & 75 & -26 \\
Phenyl- & 1750 bis 2300 & 1,4 & 5,7 & 1780 & 92 & 100 & -92 \\
acetylen & & & & & & & \\
Acetylen & 1800 bis 2200 & 0,9 & 5,7 & 1800 & 62 & 140 & -40 \\
Acetylen & 1600 bis 2250 & 1,7 & 2,6 bis 5,7 & 1800 & 82 & 150 & -28 \\
Acetylen & 1600 bis 2400 & 3,8 & 5,7 & 1800 & 90 & 110 & -25 \\
Ethylen & 1700 bis 2100 & 4,0 & 2,5 & 1825 & 48 & 150 & -70 \\
Ethylen & 1700 bis 2200 & 4,0 & 5,0 & 1850 & 69 & 145 & -71 \\
Ethylen & 1700 bis 2100 & 4,2 & 11 & 1840 & 90 & 155 & -70 \\
Ethylen & 1700 bis 2300 & 4,7 & 5,0 & 1860 & 79 & 140 & -60 \\
Ethylen & 1650 bis 2450 & 7,4 & 5,0 & 1870 & 90 & 140 & -46 \\
Methan & 1950 bis 2100 & 1,7 & 5,5 & 1970 & 14 & 130 & -60 \\
Methan & 1700 bis 2300 & 3,4 & 2,5 bis 12 & 1950 & 54 & 130 & -63 \\
Methan & 1700 bis 2400 & 6,4 & 5,5 & 1960 & 60 & 108 & -38 \\
n-Hexan & 1700 bis 2250 & 5,3 & 5,0 & 1950 & 45 & 110 & -45 \\
\hline
\end{tabular}

Tabelle 3.3: Die vier Parameter zur Berechnung der Rußausbeute nach dem Drei-SpeziesModell für verschiedene Kohlenwasserstoffe. In den ersten vier Spalten sind die experimentellen Bedingungen angegeben. 


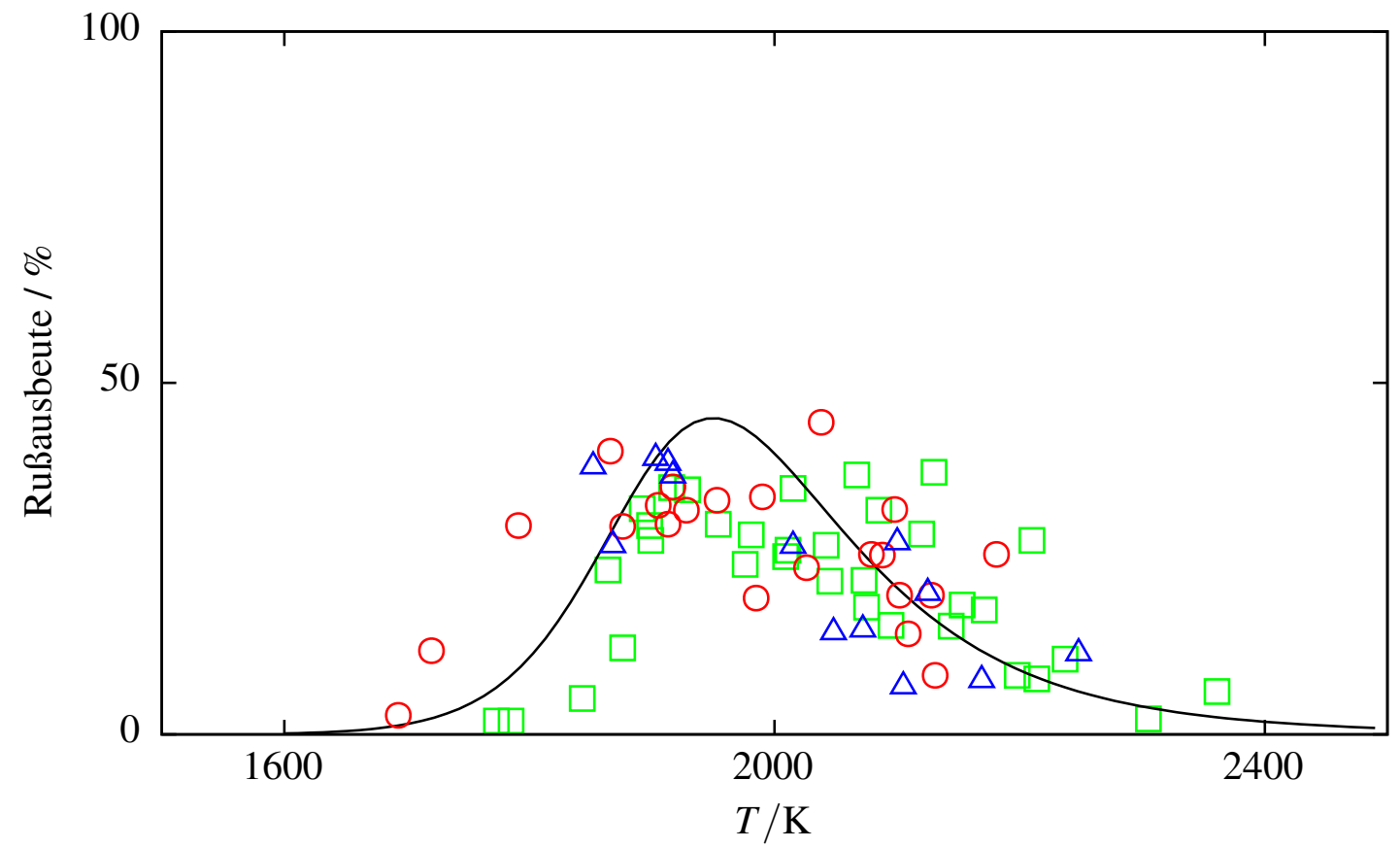

Abbildung 3.32: Temperaturabhängigkeit der Rußausbeute in der Hexanpyrolyse. $[C]$ $=5,3 \mathrm{~mol} / \mathrm{m}^{3}, \square: 2,5, \circ: 6$ und $\triangle: 10 \mathrm{MPa}$.

\subsubsection{Einfluß der Kohlenstoffkonzentration}

Der Rußausbeute nimmt mit zunehmender Kohlenstoffkonzentration zu. Im dem Temperaturbereich über $T_{\max }^{R A}$ läßt sich diese Abhängigkeit mit dem Ansatz in Gleichung (3.11) beschreiben.

$$
R A_{\infty} \propto[C]^{n}, \quad f_{v \infty} \propto[C]^{n+1} \quad \text { für } \quad T>T_{\max }^{R A}
$$

Eine beste Anpassung an die Meßwerte wird mit $n=1$ für Acetylen und Benzol und mit $n=2$ für Ethylen und Methan erreicht. Mit zunehmender Rußausbeute nimmt die Abhängigkeit von der Kohlenstoffkonzentration ab $(n \rightarrow 0)$.

\subsection{Additiv Eisenpentacarbonyl}

In diesen Experimenten wurde die Wirkung metallhaltiger Zusätze auf die Rußbildung in der Kohlenwasserstoffpyrolyse untersucht. Dazu wurden hinter reflektierten Stoßwellen bei konstantem Druck von 5,5 MPa Testgase pyrolysiert, die neben 7000 ppm Ethylen zusätzlich 200 ppm Eisenpentacarbonyl $\left(\mathrm{Fe}(\mathrm{CO})_{5}\right)$ enthielten. In Vorversuchen wurde festgestellt, daß Testgase, die nur 200 ppm Eisenpentacarbonyl aber kein Kohlenwasserstoff enthielten, bei vergleichbaren Temperaturen und Drücken keine Lichtabsorption verursachten. Abbildung (3.33) zeigt die ersten 1,3 ms eines charakteristischen Rußausbeute-Zeitprofil bei einer Temperatur von $1810 \mathrm{~K}$ und einer Kohlenstoffkonzentration von $5,3 \mathrm{~mol} / \mathrm{m}^{3}$. Das Rußmassenwachstum verlief in zwei Stufen.

Die erste Stufe der Rußbildung setzte nach einer Induktionszeit von nur ca. $40 \mu$ s, die zweite Stufe nach ca. $600 \mu$ s ein. Im einem Experiment ohne Eisenpentacarbonyl wäre unter sonst gleichen Bedingungen eine Induktionszeit von ca. $600 \mu \mathrm{s}$ zu erwarten gewesen. 


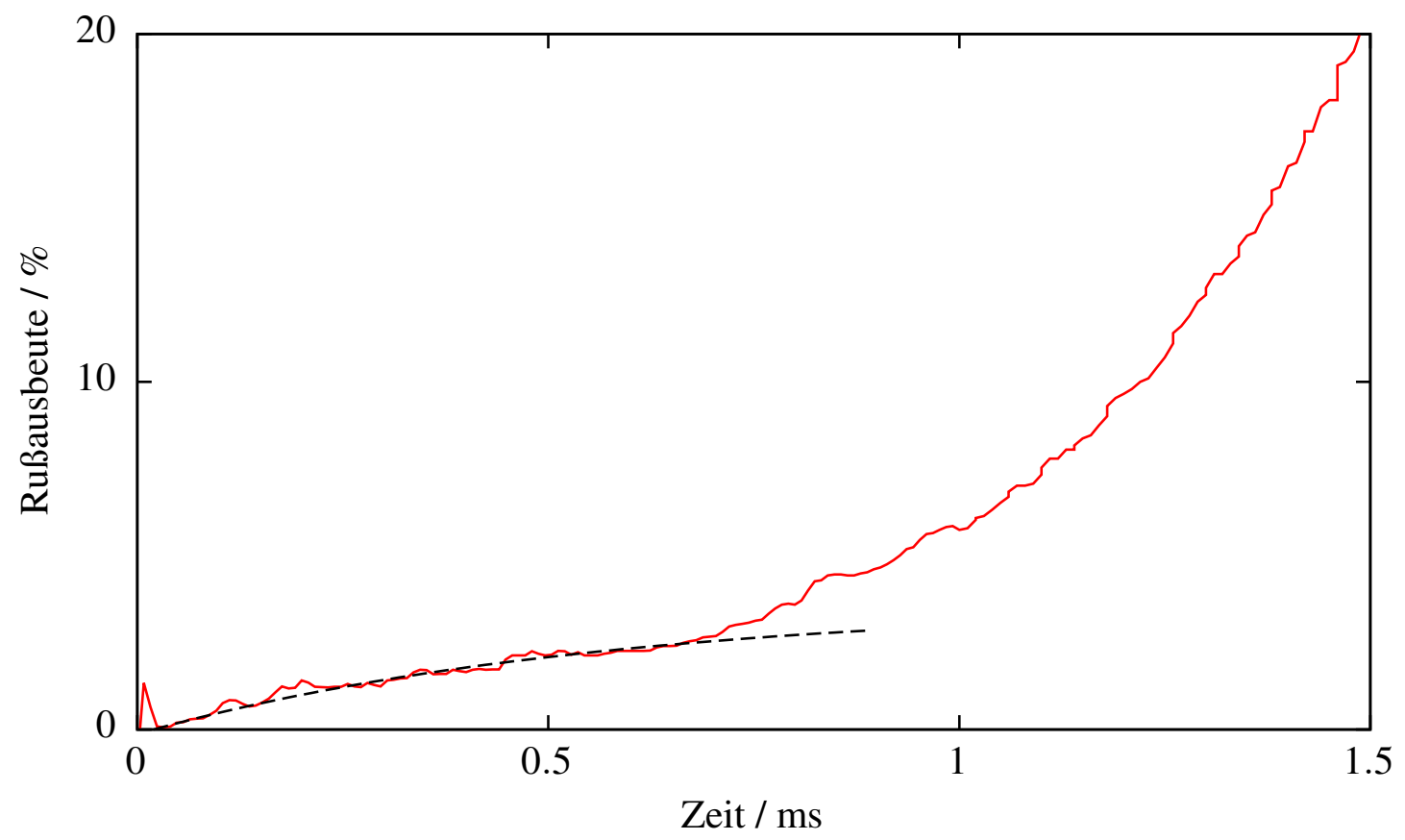

Abbildung 3.33: Ausschnitt eines Rußausbeutezeitprofils. Pyrolysiert wurde ein Eisenpentacarbonyl/Ethylen-Gemisch bei $1810 \mathrm{~K}$ und 5,7 MPa. Die Mischung enthielt $200 \mathrm{ppm}$ Eisenpentacarbonyl und $7000 \mathrm{ppm}$ Ethylen, $[C]=5,3 \mathrm{~mol} / \mathrm{m}^{3}$. Der erste Abschnitt des Rußmassenwachstums, das vor der „normalen“ Induktionszeit stattfindet, wird von einer Wachtumsfunktion pseudo-erster Ordnung mit den Parametern $k=1800 / \mathrm{s}$ und $R A=3,6 \%$ beschrieben (gestrichelte Kurve).

Unter der Wirkung von Eisenpentacarbonyl wurde offensichtlich der Beginn der Rußbildung stark gefördert. Die bis zum Einsetzen der ,normalen“ Induktionszeit gebildete Rußausbeute $(R A=3,6 \%$ ist relativ klein. Dieser Abschnitt des Profils wurde mit einer Wachstumsfunktion pseudo-erster Ordnung angepaßt. Man erhielt eine Geschwindigkeitskonstante von $(k=1800 / \mathrm{s})$. Das weitere Rußmassenwachstum setzte sich mit der für Ethylen typischen Induktionszeit und Rußmassenwachstumskonstante fort. Die am Ende erreichte Rußausbeute war mit ca. $90 \%$ um 1/3 höher als bei Experimenten ohne Eisenpentacarbonyl. Diese Rußbildung vor der „,normalen“ Induktionszeit wurde in jedem Experiment in dem Temperaturintervall zwischen 1500 und $1850 \mathrm{~K}$ beobachtet. Für diese erste Stufe der Rußbildung waren die Induktionszeiten, Massenwachstumskonstanten und Rußausbeuten temperaturunabhängig. Die Induktionszeiten und Massenwachstumskonstanten des ,normalen“ Rußmassenwachstums wurden von dem EisenpentacarbonylAdditiv nicht beeinflußt. Im Gegensatz dazu nahm die Rußausbeute im dem Temperaturbereich zwischen 1750 und $2450 \mathrm{~K}$ unter dem Einfluß von Eisenpentacarbonyl um ca. 1/3 zu. Unmittelbar hinter der reflektierten Stoßwelle lag das Eisen in Form von Clustern vor, die jeweils aus mehreren tausend Eisenatomen aufgebaut waren [22]. Die Cluster waren unmittelbar vorher, hinter der einfallenden Stoßwelle, aus Eisenpentacarbonyl über schnelle Koagulation entstanden. Es lag also ein zweistufiger Prozeß vor. Hinter der einfallenden Welle wurden Eisen-Cluster produziert, die dann den Rußbildungsprozeß hinter der reflektierten Stoßwelle beeinflußten.

Es wurden zusätzlich Experimente hinter einfallenden Stoßwellen ausgeführt. Da der Druck hinter einfallenden Stoßwellen im Mittel nur 0,6 MPa betrug, wurde in diesen Ex- 
perimenten der Ethylenanteil im Testgas auf $3 \%$ erhöht. Im Unterschied zu den Experimenten hinter reflektierten Stoßwellen lagen hier zu Beginn der Pyrolyse Eisenpentacarbonyl und Ethylen aber jedoch keine Cluster vor. Es wurden auch hier bei Temperaturen unter $1750 \mathrm{~K}$ kurze Induktionszeiten von $300 \mu$ s und kleine Rußausbeuten $(\approx 10 \%)$ beobachtet.

\subsection{Gasförmige Endprodukte}

Die Messergebnisse der Rußausbeute im Kapitel (3.4) zeigen, daß in keiner der ausgeführten Kohlenwasserstoffpyrolysen eine Rußausbeute von $100 \%$ erreicht wurde. Deswegen ist es von Interesse die anderen Pyrolyseprodukte zu analysieren. Dazu wurde am Ende der Kohlenwasserstoffpyrolyse über ein schnelles Ventilsystem eine kleine Probe des Testgases entnommen und anschließend gaschromatographisch auf $C_{1}$ bis $C_{3}$ Kohlenwasserstoffe und Wasserstoff untersucht. Aus den integrierten Peakflächen des GC-Schreibers, den GC-Eichungen und der gesamten Kohlenstoff- und Wasserstoffdichte wurden die Kohlenwasserstoff- und Wasserstoffausbeuten berechnet.

Äquivalent zur Definition der Rußausbeute wurde hier die Kohlenstoff-Ausbeute definiert als der Anteil des gesamten Kohlenstoffs, der am Ende der Pyrolyse in einem Kohlenwasserstoff gebunden ist. Entsprechend wurde die Wasserstoff-Ausbeute definiert:

$$
\begin{aligned}
& \text { Kohlenstoff-Ausbeute }=n \cdot \frac{\left[\mathrm{C}_{n} \mathrm{H}_{m}\right]}{[\mathrm{C}]_{\text {total }}} \\
& \text { Wasserstoff-Ausbeute }=m \cdot \frac{\left[\mathrm{C}_{n} \mathrm{H}_{m}\right]}{[\mathrm{H}]_{\text {total }}}
\end{aligned}
$$

In Vorversuchen wurde zunächst erfolgreich getestet, daß sich kein Wasserstoff aus dem Treibgas in der Probe befindet und daß die Wiederfindungsquote der Referenzsubstanz Helium $100 \%$ beträgt.

Pyrolysiert wurde Benzol, Ethylen und n-Hexan im Temperaturintervall von 1600 bis $2300 \mathrm{~K}$ bei einem konstantem Druck von $5 \mathrm{MPa}$. Die mittleren Kohlenstoffkonzentrationen betrugen in den Benzolpyrolysen 0,4, 1, 4 und $40 \mathrm{~mol} / \mathrm{m}^{3}$, in den Hexanpyrolysen 5,3, 20 und $40 \mathrm{~mol} / \mathrm{m}^{3}$ und in den Ethylenpyrolysen $6 \mathrm{~mol} / \mathrm{m}^{3}$. In den folgenden Abbildungen sind die Ausbeuten von Methan, Acetylen, Ethylen, Ethan und Wasserstoff als Funktion der Temperatur dargestellt.

\subsubsection{Methan-Ausbeute}

Abbildung (3.34) zeigt die Methan-Ausbeute in der Benzol-Pyrolyse. Mit zunehmender Temperatur nimmt die Methan-Ausbeute von 2,5 auf 0,2\% ab. Obwohl die Benzolkonzentration um zwei Größenordnungen variiert wurde, wurde kein Einfluß der Benzolkonzentration festgestellt. Im Gegensatz dazu nimmt die Methan-Ausbeute in der Hexanpyrolyse mit zunehmender Kohlenstoffdichte zu, siehe Abbildung (3.35). Bei einer Kohlenstoffkonzentration von $(5 \pm 1) \mathrm{mol} / \mathrm{m}^{3}$ ist die Methan-Ausbeute in der Ethylenpyrolyse $50 \%$ höher, in der Hexanpyrolyse dreifach höher als in der Benzolpyrolyse. 


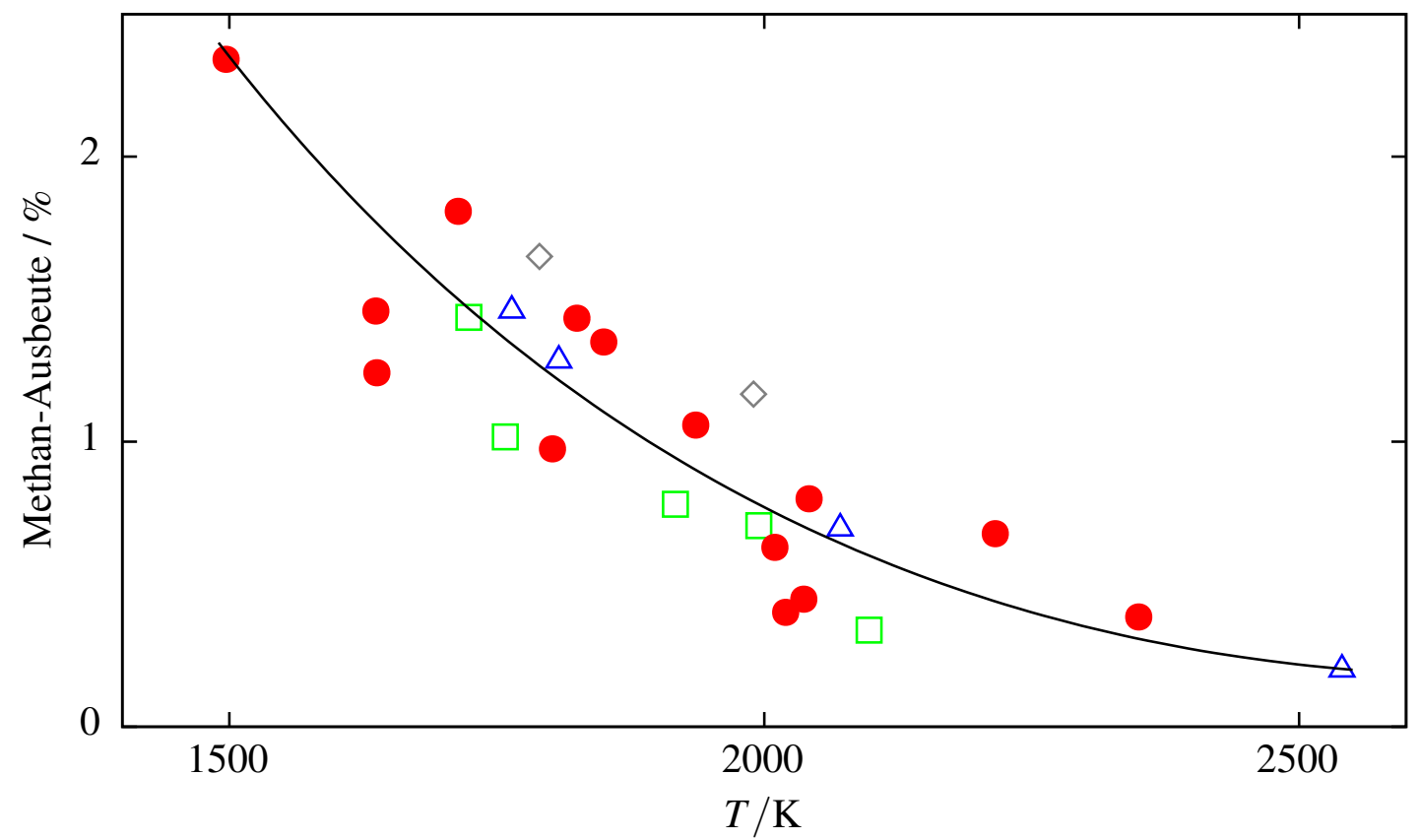

Abbildung 3.34: Temperaturabhängigkeit der Methan-Ausbeute in der Benzolpyrolyse. Die Kohlenstoffkonzentrationen betragen: $\diamond: 0,4, \square: 1, \bullet: 4$ und $\triangle: 40 \mathrm{~mol} / \mathrm{m}^{3}$.

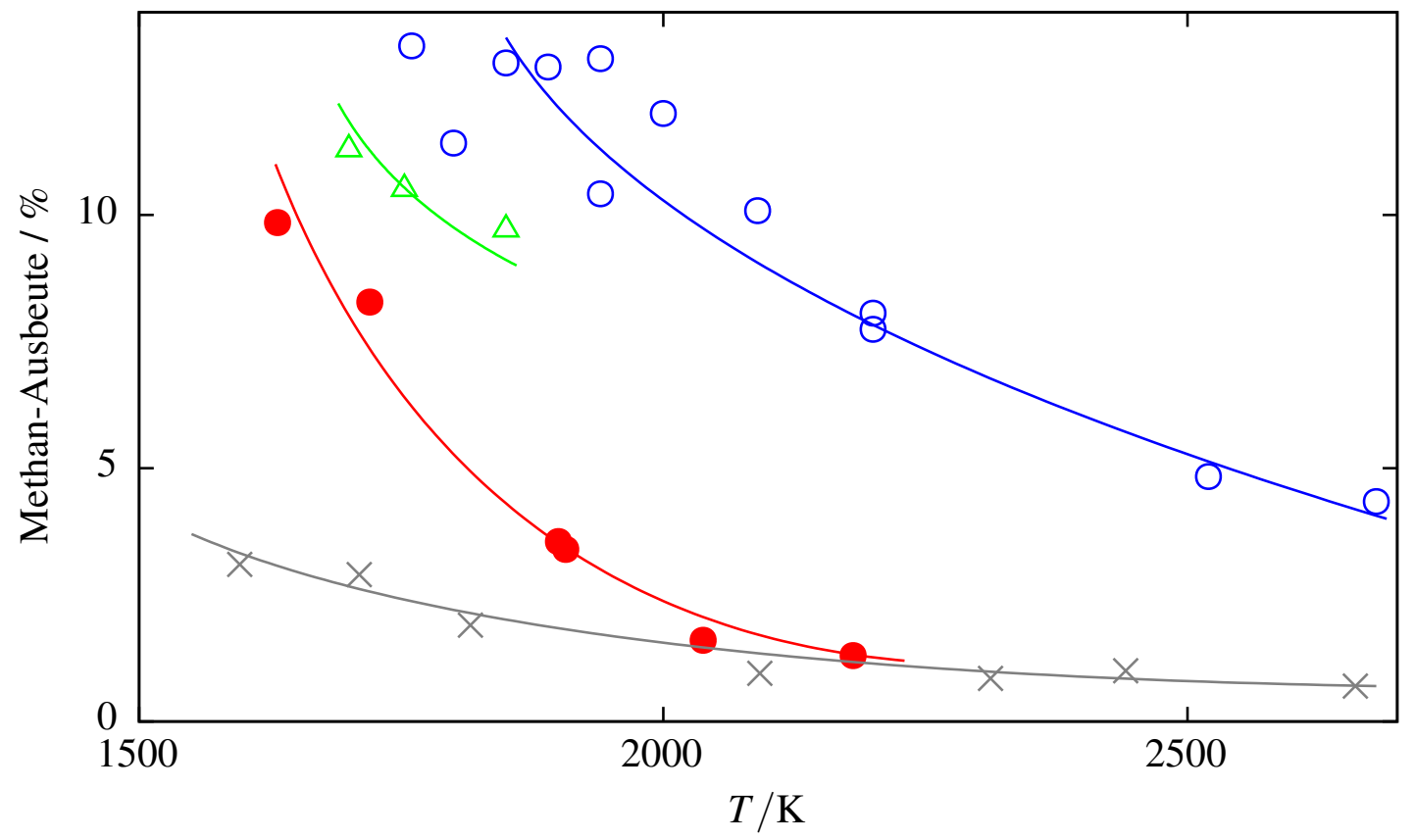

Abbildung 3.35: Temperaturabhängigkeit der Methan-Ausbeute in der n-Hexanund Ethylenpyrolyse. Die Kohlenstoffkonzentrationen betragen: $\bullet$ : 5,3, $\triangle$ : 20 und $\circ: 40 \mathrm{~mol} / \mathrm{m}^{3}$ in der Hexanpyrolyse und $\times: 6 \mathrm{~mol} / \mathrm{m}^{3}$ in der Ethylenpyrolyse. 


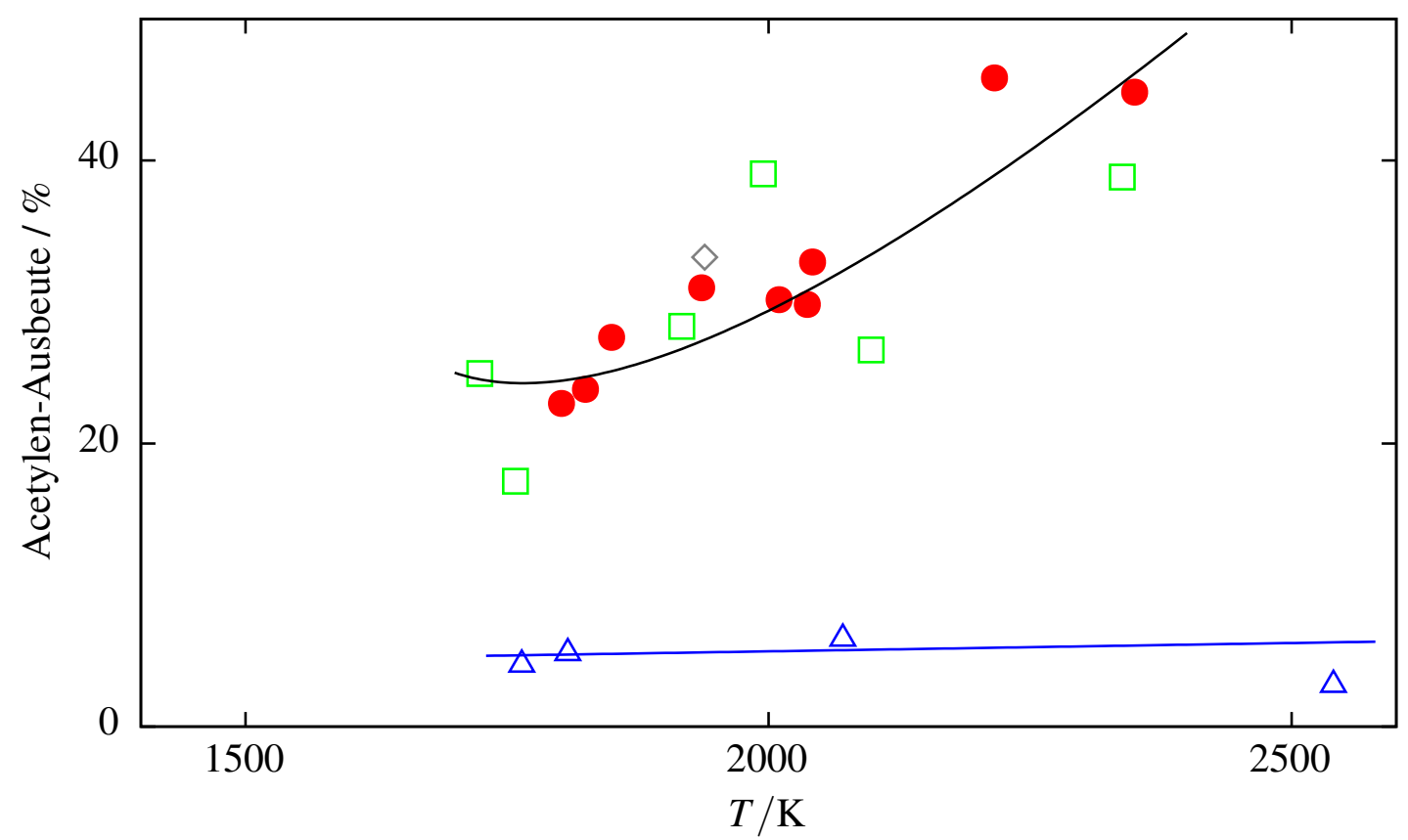

Abbildung 3.36: Temperaturabhängigkeit der Acetylen-Ausbeute in der Benzolpyrolyse. Die Kohlenstoffkonzentrationen betragen: $\diamond: 0,4, \square: 1, \bullet: 4$ und $\triangle: 40 \mathrm{~mol} / \mathrm{m}^{3}$.

\subsubsection{Acetylen-Ausbeute}

Die Ergebnisse der Acetylen-Ausbeuten (Abbildungen (3.36) und (3.37)) unterscheiden sich sehr stark von den Methan-Ausbeuten. Bei einer Kohlenstoffkonzentration von $(5 \pm 1) \mathrm{mol} / \mathrm{m}^{3}$ haben die Acetylen-Ausbeuten in Kohlenwasserstoffpyrolysen an der Temperatur ein Minimum, an der die Rußausbeute ein Maximum hat. In der Hexanpyrolyse beträgt die Acetylen-Ausbeute bei $1900 \mathrm{~K} 45 \%$. Für Ethylen und Benzol wurde bei dieser Temperatur eine Ausbeute von $38 \%$ bzw. $28 \%$ gemessen. Mit zunehmender Kohlenstoffdichte nimmt die Acetylen-Ausbeute ab.

\subsubsection{Ethylen-Ausbeute}

Die Ethylen-Ausbeute in der Benzolpyrolyse nimmt von $0,6 \%$ bei $1600 \mathrm{~K}$ auf $1 \%$ bei $2400 \mathrm{~K}$ nur wenig zu, siehe Abbildung (3.38). Eine Abhängigkeit von der Benzolkonzentration wurde nicht festgestellt. In der n-Hexan- und Ethylenpyrolyse zeigt die EthylenAusbeute die gleiche Charakteristik wie die Methan-Ausbeute, siehe Abbildung (3.39). Die Ausbeuten nehmen mit abnehmender Temperatur und zunehmender Kohlenstoffdichte zu. In der Hexanpyrolyse wurde etwas mehr Ethylen nachgewiesen als in der Ethylenpyrolyse. Im Vergleich zu Benzol sind die Ausbeuten erheblich höher.

\subsubsection{Ethan-Ausbeute}

Die Ethan-Ausbeuten sind unter allen untersuchten Bedingungen kleiner als $2 \%$. Für Experimente mit geringer Kohlenstoffdichte lagen die Ethan-Ausbeuten im Bereich der Nachweisgrenze. In Abbildung (3.40) sind die Ergebnisse der Benzol- und Hexanpyrolysen dargestellt. Die Ethan-Ausbeute nimmt wie die Methan-Ausbeute mit steigender Temperatur ab. 


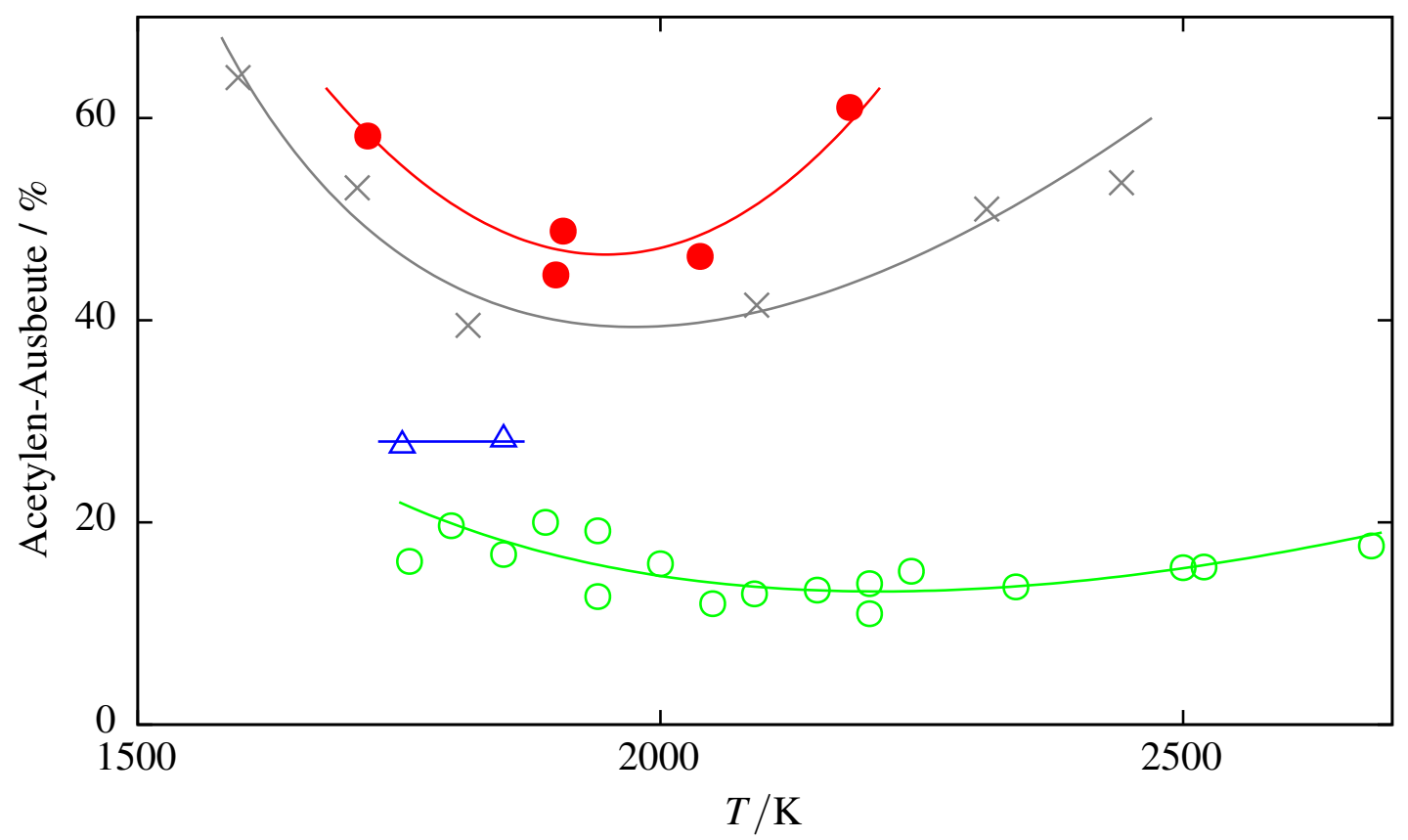

Abbildung 3.37: Temperaturabhängigkeit der Acetylen-Ausbeute in der n-Hexanund Ethylenpyrolyse. Die Kohlenstoffkonzentrationen betragen: $\bullet$ : 5,3, $\triangle$ : 20 und $\circ: 40 \mathrm{~mol} / \mathrm{m}^{3}$ in der Hexanpyrolyse und $\times: 6 \mathrm{~mol} / \mathrm{m}^{3}$ in der Ethylenpyrolyse.

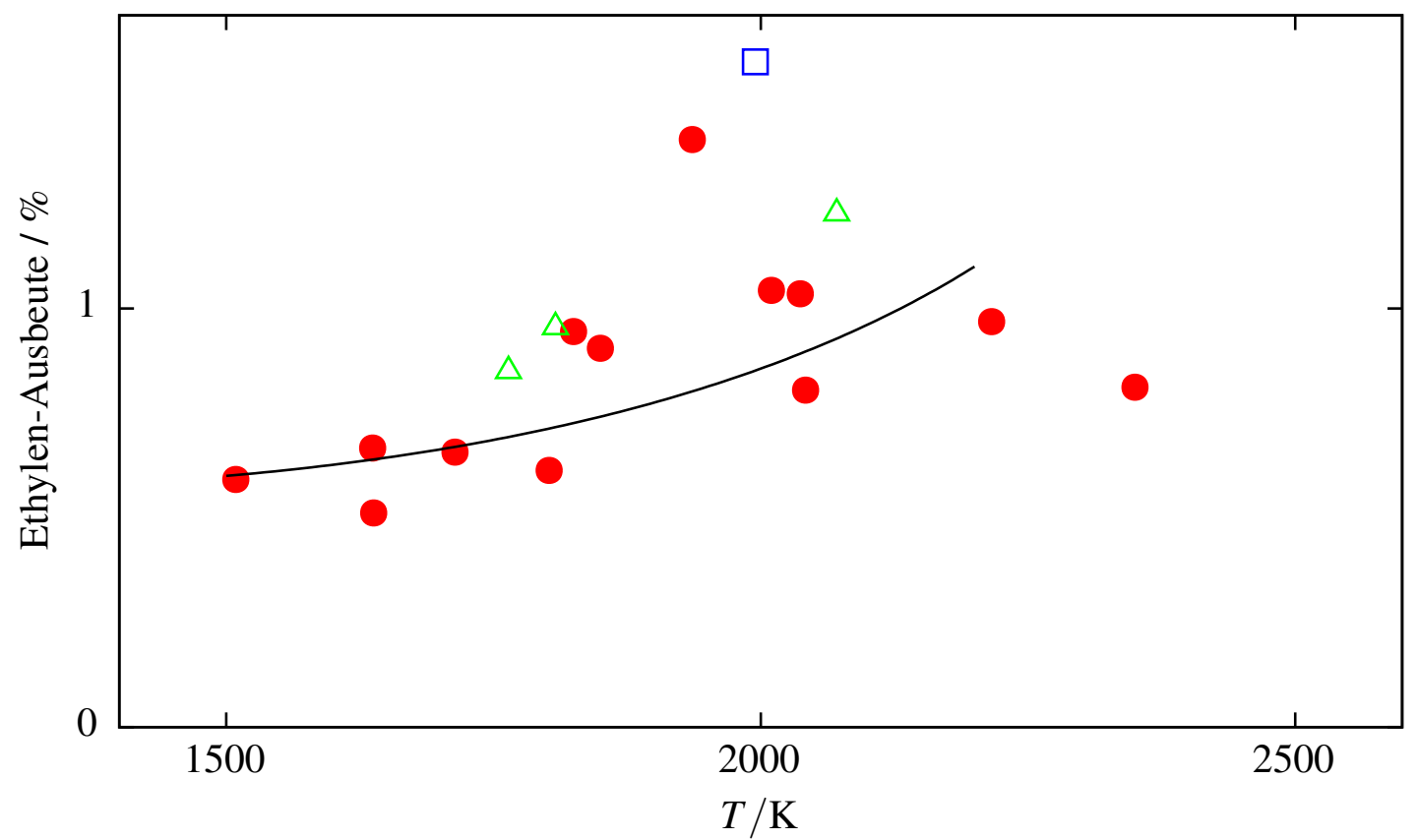

Abbildung 3.38: Temperaturabhängigkeit der Ethylen-Ausbeute in der Benzolpyrolyse. Die Kohlenstoffkonzentrationen betragen: $\square: 1, \bullet: 4$ und $\triangle: 40 \mathrm{~mol} / \mathrm{m}^{3}$. 


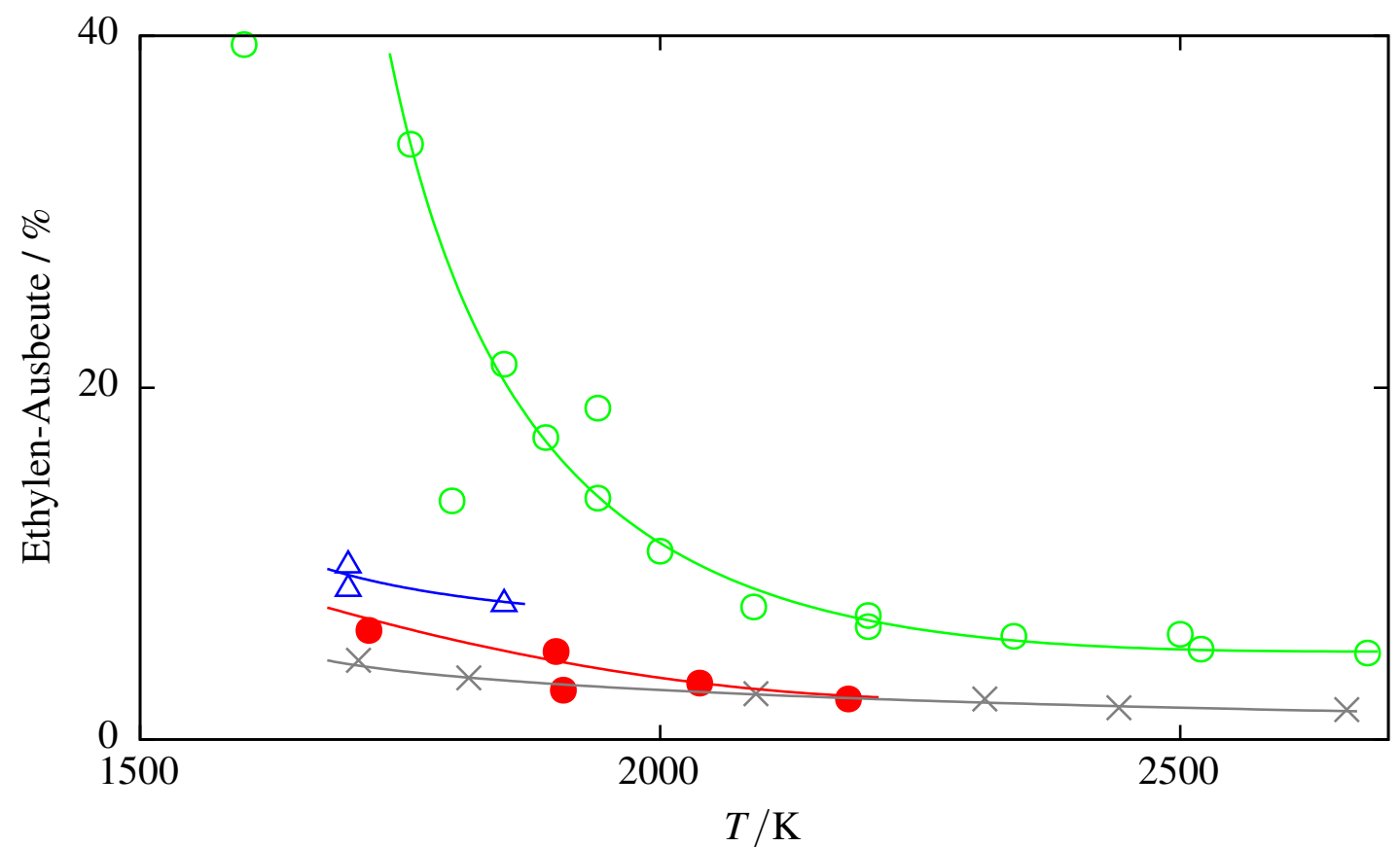

Abbildung 3.39: Temperaturabhängigkeit der Ethylen-Ausbeute in der n-Hexanund Ethylenpyrolyse. Die Kohlenstoffkonzentrationen betragen: •: 5,3, $\triangle$ : 20 und $\circ: 40 \mathrm{~mol} / \mathrm{m}^{3}$ in der Hexanpyrolyse und $\times: 6 \mathrm{~mol} / \mathrm{m}^{3}$ in der Ethylenpyrolyse.

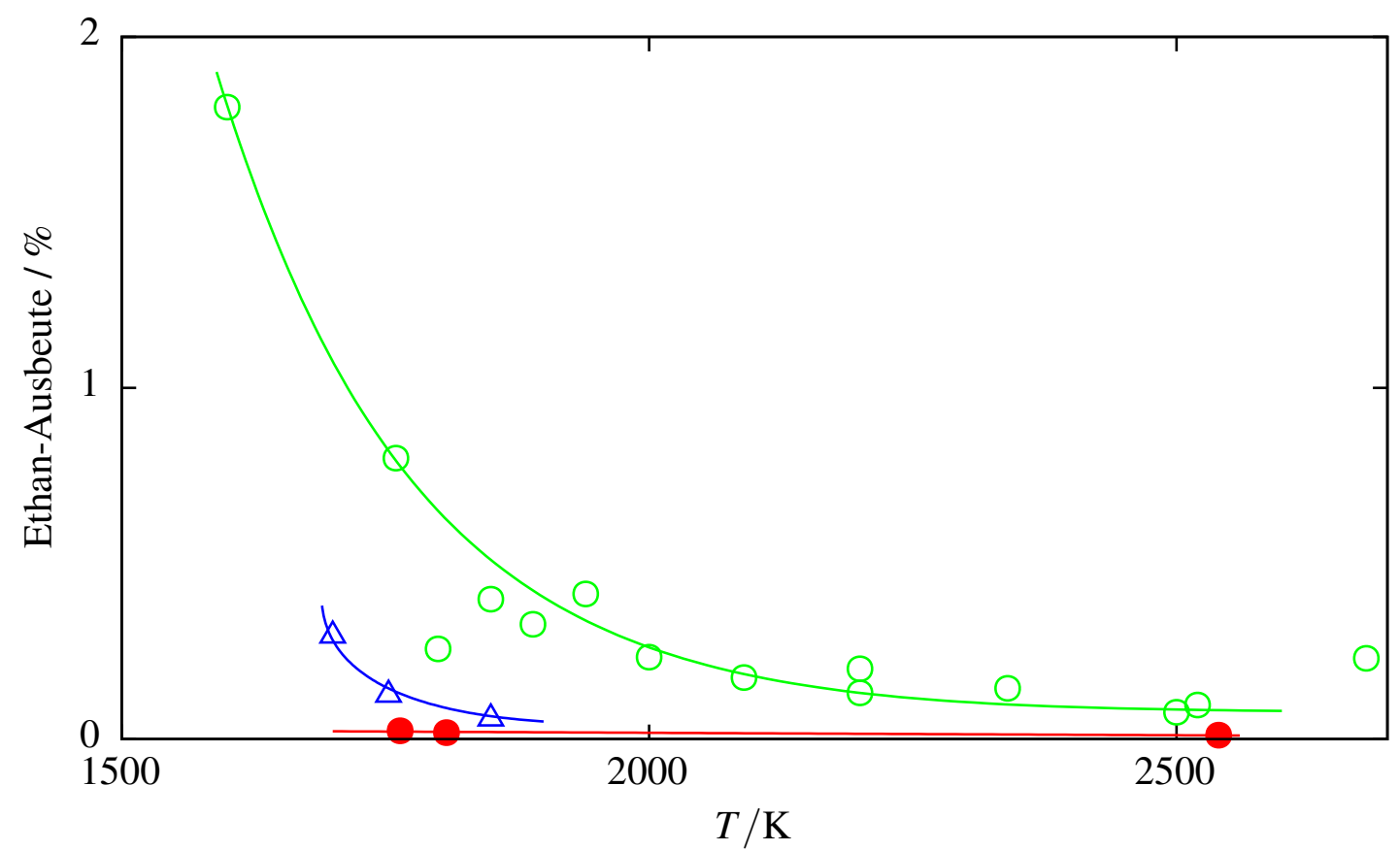

Abbildung 3.40: Temperaturabhängigkeit der Ethan-Ausbeute in der n-Hexan- und Benzolpyrolyse. Die Kohlenstoffkonzentrationen betragen: $\triangle: 20$ und $\circ: 40 \mathrm{~mol} / \mathrm{m}^{3}$ in der Hexanpyrolyse und $\bullet: 40 \mathrm{~mol} / \mathrm{m}^{3}$ in der Benzolpyrolyse. 


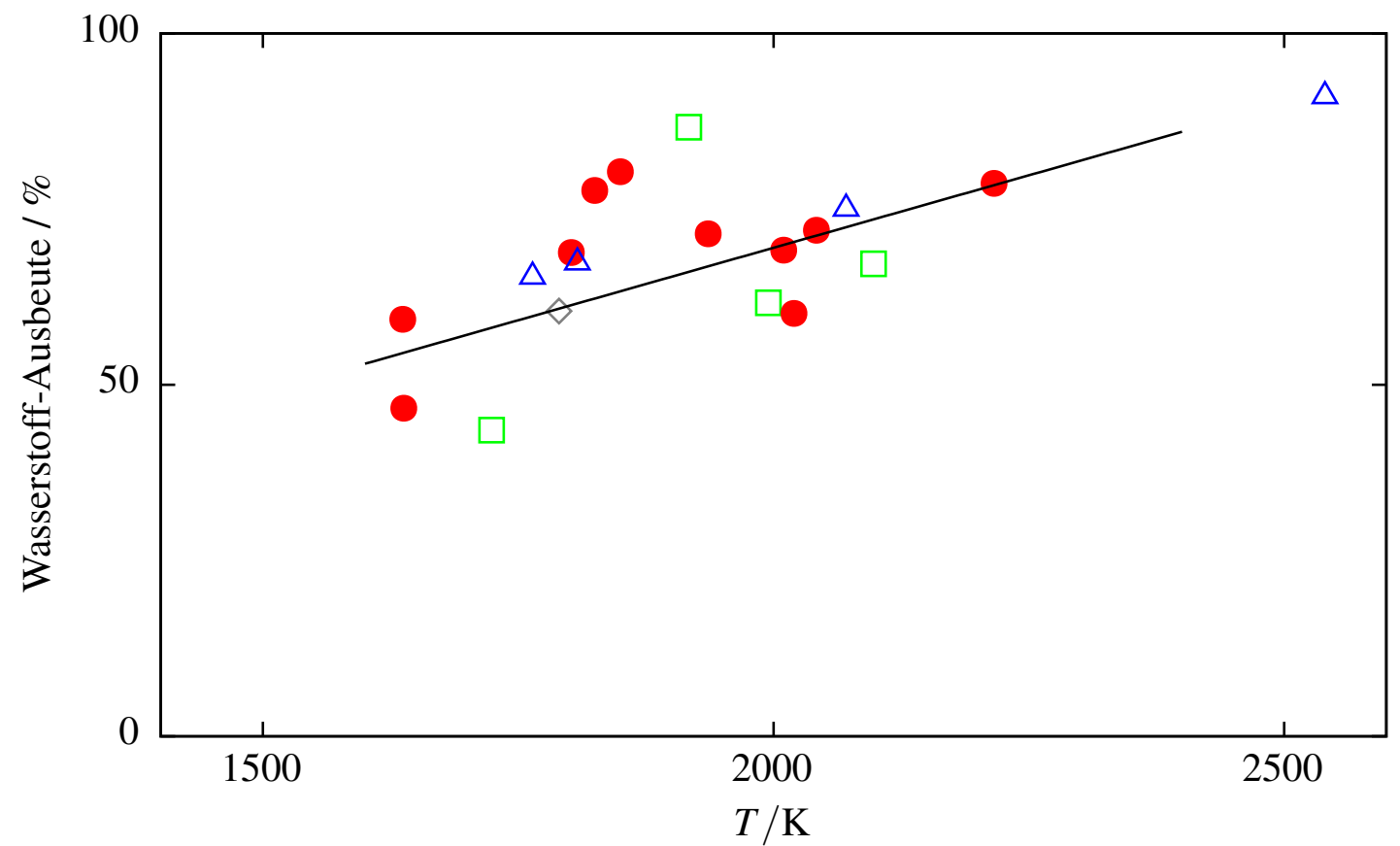

Abbildung 3.41: Temperaturabhängigkeit der Wasserstoff-Ausbeute in der Benzolpyrolyse. Die Kohlenstoffkonzentrationen betragen: $\diamond: 0,4, \square: 1, \bullet: 4$ und $\triangle: 40 \mathrm{~mol} / \mathrm{m}^{3}$.

\subsubsection{Wasserstoff-Ausbeute}

In Kohlenwasserstoffpyrolysen (Abbildung (3.41) und (3.42)) nimmt die Ausbeute von $50 \%$ bei $1700 \mathrm{~K}$ auf $75 \%$ bei $2200 \mathrm{~K}$ linear zu. Nur in Pyrolysen mit hoher Hexankonzentration ist die Wasserstoff-Ausbeute um ca. $20 \%$ niedriger.

\subsubsection{Höhere Kohlenwasserstoffe}

In keiner Testgasprobe wurden $C_{3}$ Kohlenwasserstoffe nachgewiesen. Das heißt, daß die Ausbeuten deutlich unter $1 \%$ liegen müssen. Polycyclische Aromaten wurden mittels HPLC analysiert (siehe unten). In Massenbilanzen spielen polycyclische Aromaten nur eine vernachlässigbar kleine Rolle.

\subsubsection{Wasserstoff-Massenbilanz}

Die Wiederfindungsquote der Referenzsubstanz war 100\%. Daraus wurde geschlossen, daß die Proben keinen Wasserstoff aus dem Treibgas enthielten. Falls alle wasserstoffenthaltenden Spezies quantitativ nachgewiesen wurden, sollte die Wiederfindungsquote $100 \%$ betragen. Aus den Methan-, Acetylen-, Ethylen-, Ethan- und Wasserstoff-Ausbeuten wurden Wasserstoff-Massenbilanzen berechnet. Das Ergebnis ist in den Abbildungen (3.43) und (3.44) dargestellt. Bis auf die Pyrolysen mit hoher Kohlenstoffdichte wurden rund $100 \%$ des eingesetzten Wasserstoffs in den Reaktionsprodukten wiedergefunden. In Pyrolysen mit $2 \%$ Benzol und $2 \%$ n-Hexan war die Wiederfindungsquote bis zu $15 \%$ niedriger. 


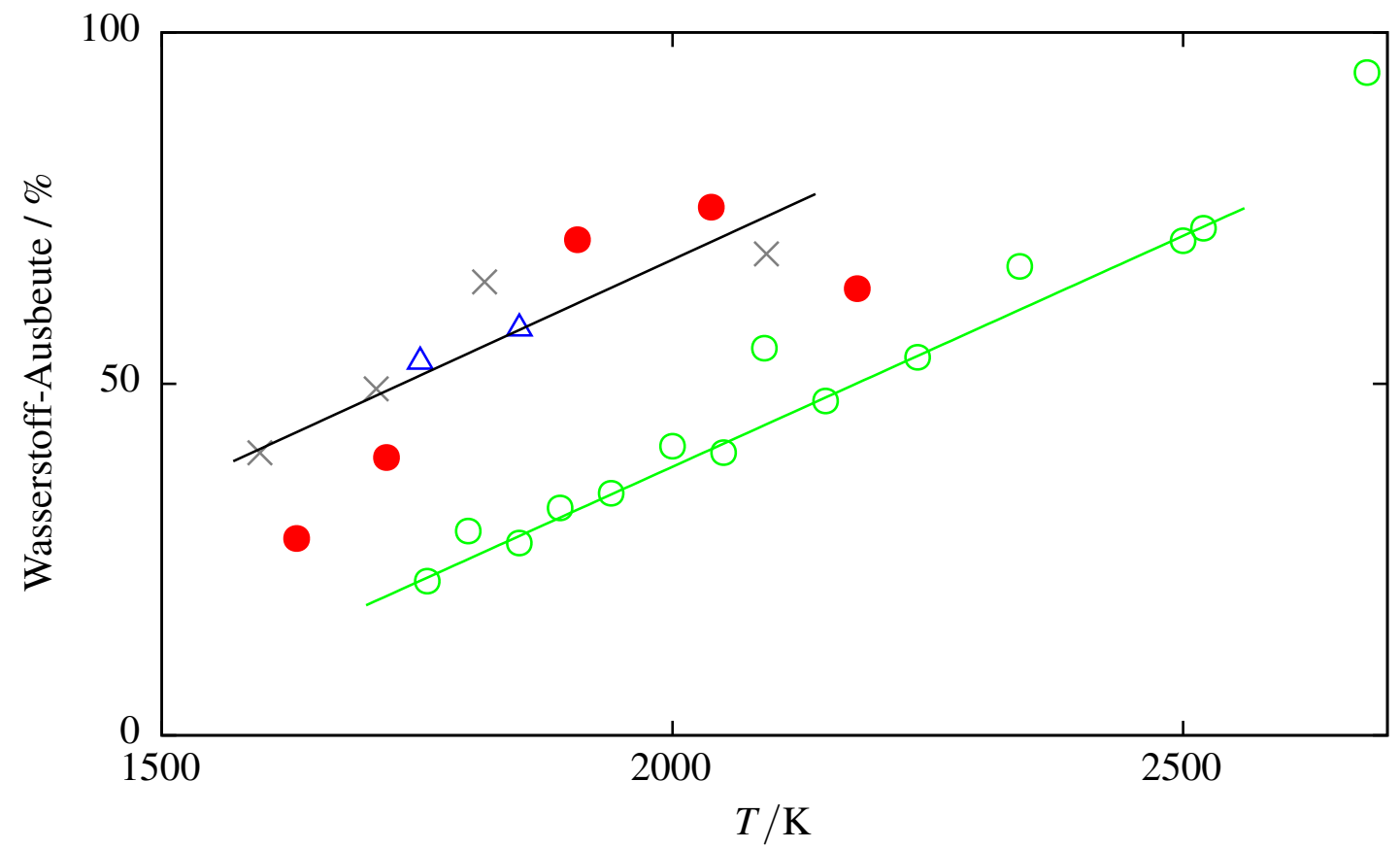

Abbildung 3.42: Temperaturabhängigkeit der Wasserstoff-Ausbeute in der n-Hexanund Ethylenpyrolyse. Die Kohlenstoffkonzentrationen betragen: $\bullet$ : 5,3, $\triangle$ : 20 und $\circ: 40 \mathrm{~mol} / \mathrm{m}^{3}$ in der Hexanpyrolyse und $\times: 6 \mathrm{~mol} / \mathrm{m}^{3}$ in der Ethylenpyrolyse.

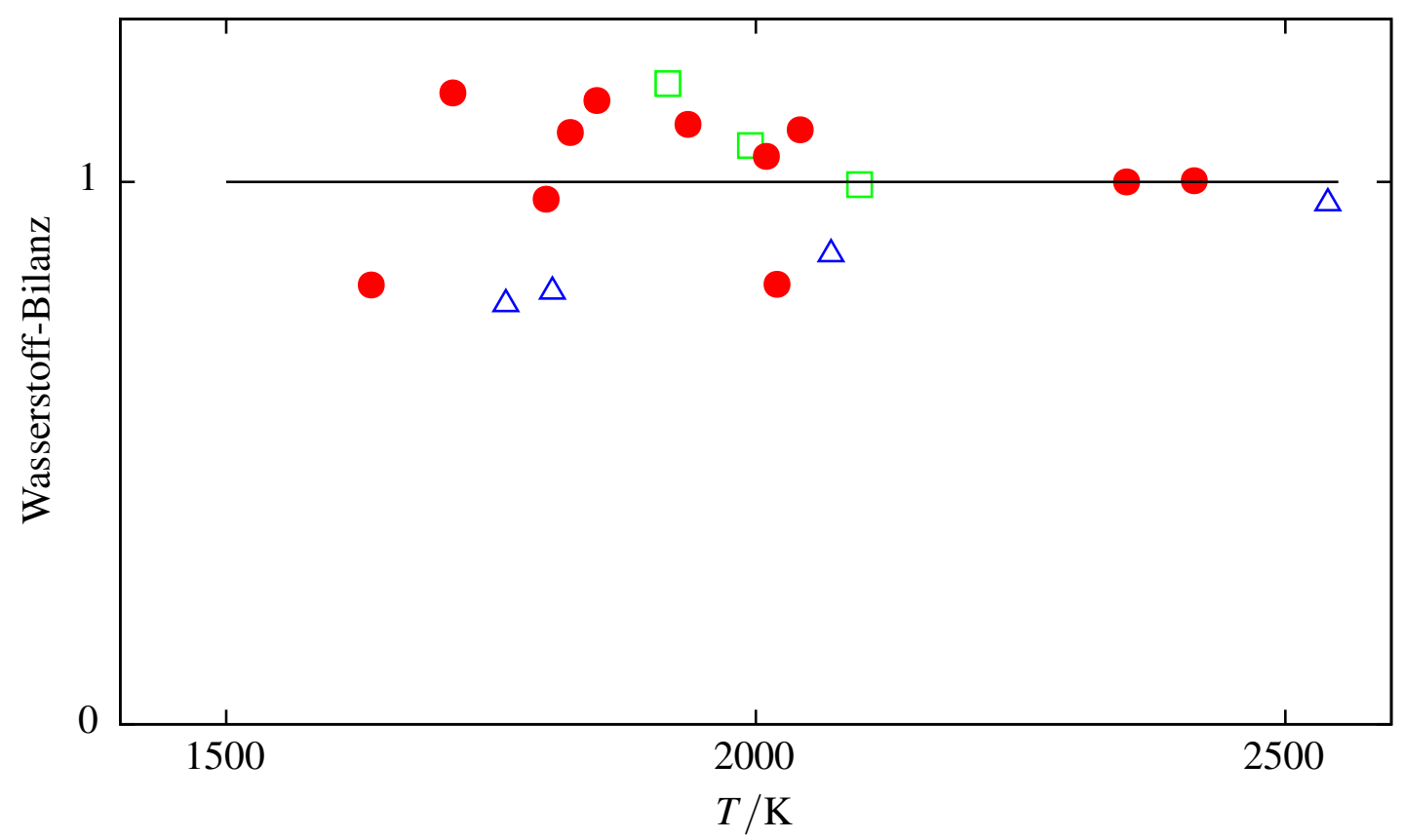

Abbildung 3.43: Temperaturabhängigkeit der Wasserstoff-Bilanz in der Benzolpyrolyse. Die Kohlenstoffkonzentrationen betragen: $\square: 1, \bullet: 4$ und $\triangle: 40 \mathrm{~mol} / \mathrm{m}^{3}$. 


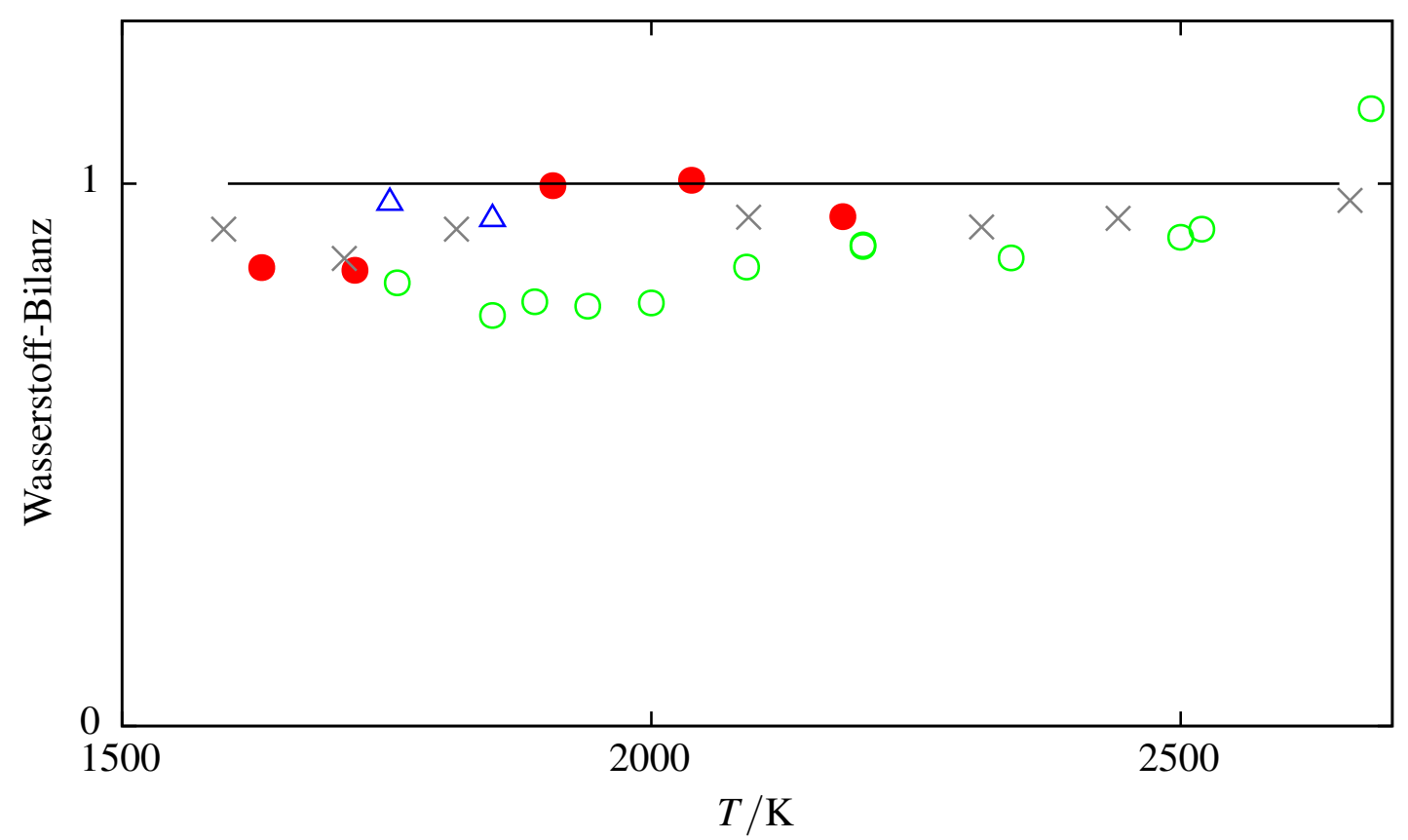

Abbildung 3.44: Temperaturabhängigkeit der Wasserstoff-Bilanz in der n-Hexanund Ethylenpyrolyse. Die Kohlenstoffkonzentrationen betragen: $\bullet$ : 5,3, $\triangle: 20$ und $\circ: 40 \mathrm{~mol} / \mathrm{m}^{3}$ in der Hexanpyrolyse und $\times: 6 \mathrm{~mol} / \mathrm{m}^{3}$ in der Ethylenpyrolyse.

\subsubsection{Kohlenstoff-Massenbilanz}

Aus der Summe Methan-, Acetylen-, Ethylen- und Ethan-Ausbeuten sowie den optisch gemessenen Rußausbeuten wurden Kohlenstoff-Gesamtmassenbilanzen berechnet. Im Mittel wurden $90 \%$ des eingesetzten Kohlenstoffs in den Reaktionsprodukten wiedergefunden, siehe Abbildung (3.45). Diese Meßergebnisse stimmen im Rahmen der Fehlergrenzen mit der theoretischen Wiederfindungsquote von $100 \%$ gut überein. Für hohe Kohlenstoffdichten konnten, mit dem in dieser Arbeit verwendeten optischen Aufbau, keine Rußausbeuten bestimmt werden. Dafür wäre im Bereich hoher Absorption mindestens eine zehnfach höhere Auflösung des Laserlichtdetektorsystems notwendig gewesen. In diesen Fall wurden die Kohlenstoff-Massenbilanzen ohne Rußausbeuten berechnet, siehe Abbildung (3.46). Bei $1900 \mathrm{~K}$ wurden in den Hexanpyrolysen unabhängig von der Hexankonzentration $50 \%$ des Kohlenstoffs in $C_{1}$ und $C_{2}$ Kohlenwasserstoffen nachgewiesen. Im Gegensatz dazu pyrolysierte ein $2 \%$ Benzol-Testgas unter sonst gleichen Bedingungen nur zu $8 \%$ zu $C_{1}$ und $C_{2}$ Kohlenwasserstoffe.

\subsubsection{Einfluß des Gesamtdruckes}

Bei konstanter Temperatur von $1930 \mathrm{~K}$ wurde ein $1 \%$ Ethylen enthaltendes Testgas bei unterschiedlichen Drücken pyrolysiert. Dieses Verfahren steht in Analogie zu Flammenexperimenten, bei denen das $(\mathrm{C} / \mathrm{O})$-Verhältnis und die Frischgasanströmgeschwindigkeit konstant gehalten werden und der Druck variiert wird. Die Ausbeuten an Kohlenwasserstoffen und Wasserstoff sind in Abbildung (3.47) dargestellt. Die Methan- und Ethylenausbeuten nahmen bei Vervierfachung des Druckes von 2,5 auf $10 \mathrm{MPa}$ auf mehr als das Doppelte zu. Die Wasserstoffausbeute nahm um ein Drittel zu, während sich die Acetylenausbeute von $60 \%$ auf $30 \%$ halbierte. 


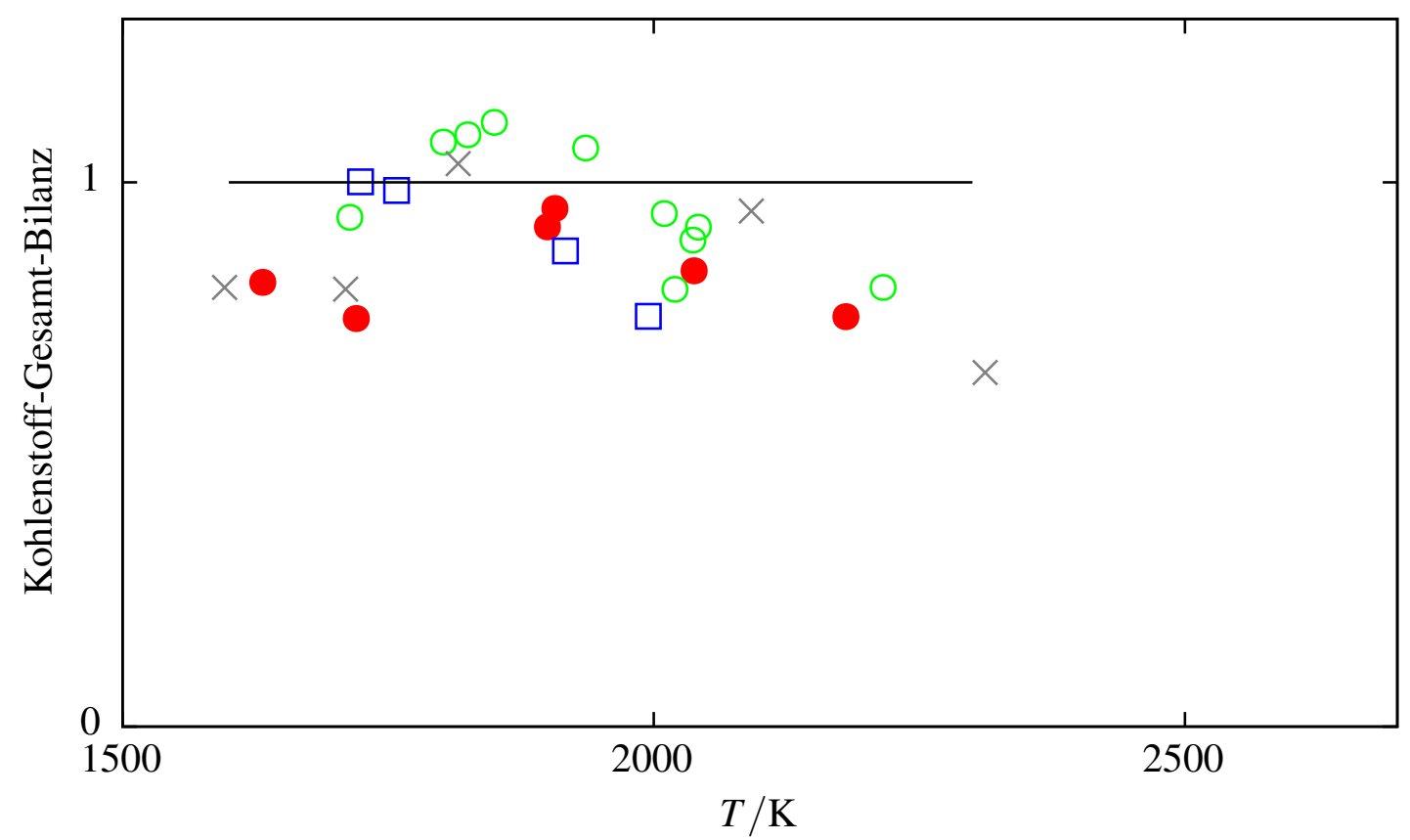

Abbildung 3.45: Temperaturabhängigkeit der Kohlenstoff-Gesamtmassenbilanz in der Benzol-, n-Hexan- und Ethylenpyrolyse. Zur Berechnung der Gesamtmassenbilanz wurden die Ausbeuten der nachgewiesenen kohlenstoffhaltigen Gasspezies und die optisch bestimmte Rußausbeuten summiert. Die Kohlenstoffkonzentrationen betragen: $\square: 1$, $\circ: 4 \mathrm{~mol} / \mathrm{m}^{3}$ in der Benzol-, $\bullet: 5,3 \mathrm{~mol} / \mathrm{m}^{3}$ in der Hexan- und $\times: 6 \mathrm{~mol} / \mathrm{m}^{3}$ in der Ethylenpyrolyse.

\subsubsection{Polycyclische Aromaten}

Aus der kleinen Gasprobe von $500 \mathrm{~cm}^{3}$ wurden die höheren Kohlenwasserstoffe quantitativ nachgewiesen. Um Konzentrationen oberhalb der HPLC-Nachweisgrenze zu erhalten, wurde ein Testgas mit relativ hohem n-Hexan-Anteil (2\%) bei einem Druck von 5,0 MPa und einer Temperatur von $2050 \mathrm{~K}$ pyrolysiert. Jedem Peak des Chromatogramms konnte eine Verbindung zugeordnet werden. Es wurden ausschließlich polycyclische aromatische Kohlenwasserstoffe ohne Seitenkette nachgewiesen. In Tabelle (3.4) sind die Molenbrüche der Aromaten dargestellt. Die Ausbeute an polycyclischen aromatischen Kohlenwasserstoffen beträgt $\frac{[C]_{\mathrm{PAH}}}{[\mathrm{C}]_{\text {total }}}=1 / 5000$. Keiner der nachgewiesenen Aromaten enthält mehr als einen Fünfring. Der Massenanteil der Aromaten mit einem Fünfring beträgt $55 \%$ von der Gesamtsumme der Aromaten.

\subsection{Elementaranalyse}

Rußproben, die aus Pyrolysen von $1 \%$ Ethylen bzw. 1\% n-Hexan bei $1900 \mathrm{~K}$ und 10,5 MPa stammen, wurden im Institut für Anorganische Chemie durch Elementaranalyse auf $C$ - und $H$-Atome untersucht. Das $(H: C)$-Molverhältnis dieser Rußproben beträgt (1:4). Das heißt, daß sich maximal $5 \%$ des gesamten Wasserstoffs im Ruß befinden. Ein Anteil des adsorbierten Wasserstoffs könnte aus dem Treibgas stammen. Experimente mit Helium als Treibgas würden diesen systematischen Fehler beseitigen. Die Proben enthielten kleine, mit dem bloßem Auge gerade noch sichtbare, Aluminium-Splitter, die aus der 


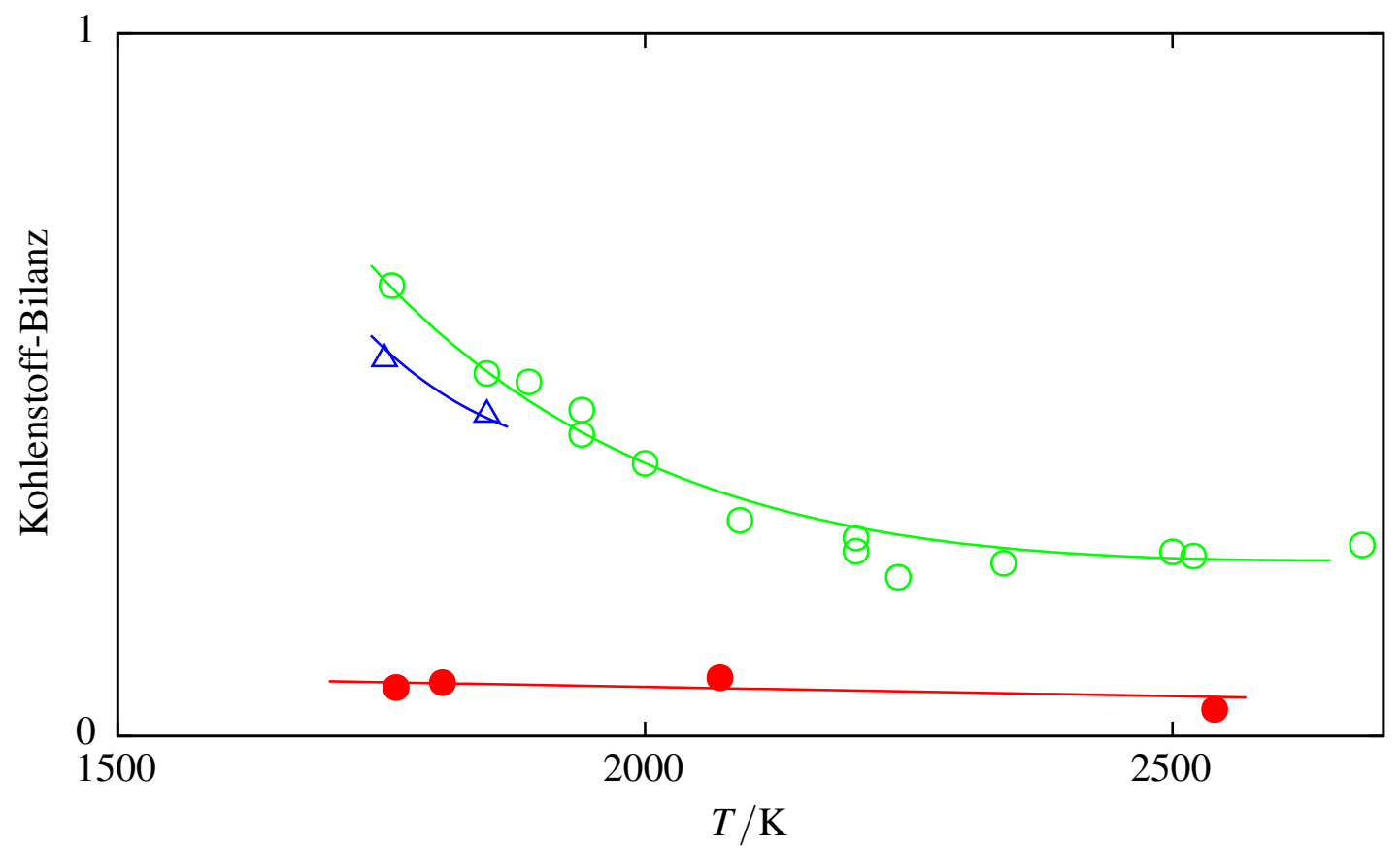

Abbildung 3.46: Temperaturabhängigkeit der Kohlenstoff-Massenbilanz in der Benzolund Hexanpyrolyse. Zur Berechnung der Massenbilanz wurden nur die Ausbeuten der nachgewiesenen kohlenstoffhaltigen Gasspezies summiert. Wegen der hohen Kohlenstoffdichten konnten in dieser Arbeit die Rußausbeuten optisch nicht bestimmt werden. Die Kohlenstoffkonzentrationen betragen: $\bullet: 40 \mathrm{~mol} / \mathrm{m}^{3}$ in der Benzol- und $\triangle: 20$, ○: $40 \mathrm{~mol} / \mathrm{m}^{3}$ in der Hexanpyrolyse.

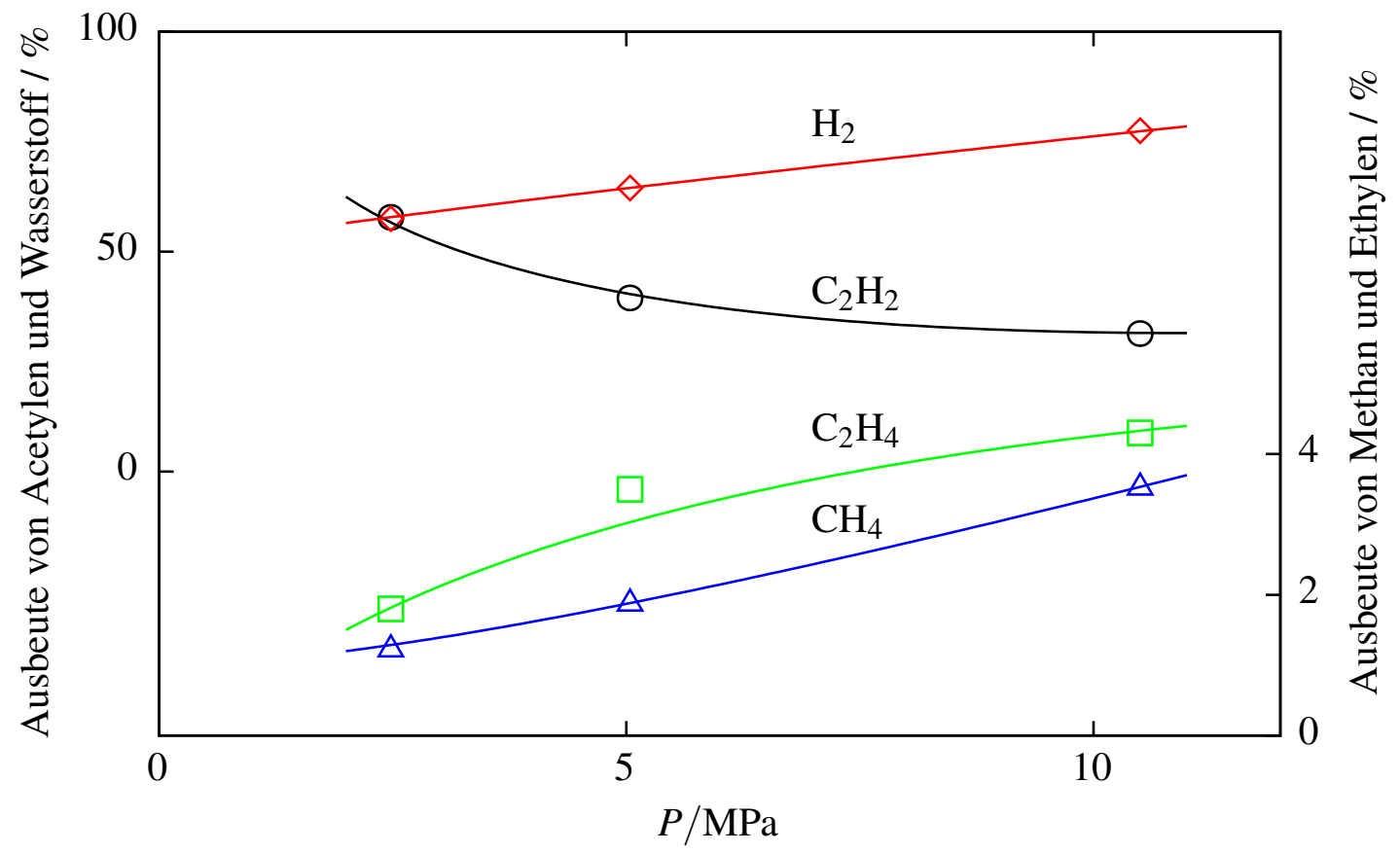

Abbildung 3.47: Kohlenwasserstoff- und Wasserstoffausbeuten in der Ethylenpyrolyse bei konstanter Temperatur $(1930 \mathrm{~K})$ und konstantem Ethylenanteil von $1 \%$ in Abhängigkeit vom Druck. $\triangle$ : Methan, $\circ$ : Acetylen, $\square$ : Ethylen und $\diamond$ : Wasserstoff. 


\begin{tabular}{lclc}
\hline PAH ohne Fünfring & $x / 10^{-8}$ & PAH mit einem Fünfring & $x / 10^{-8}$ \\
\hline Naphthalin & 39 & Acenaphthylen & 24 \\
Phenanthren & 15 & Acenaphthen & - \\
Anthracen & - & Fluoren & 16 \\
Pyren & 24 & Fluoranthen & 39 \\
Benzo(a)anthracen & - & Acephenanthrylen & 17 \\
Chrysen & 1 & Benzo(b)fluoranthen & 2 \\
Benzo(e)pyren & 2 & Benzo(k)fluoranthen & 1 \\
Benzo(a)pyren & 2 & Indeno(1,2,3-c,d)pyren & 1 \\
Dibenzo(a,h)anthracen & 3 & & \\
Benzo(g,h,i)perylen & 3 & & \\
\hline
\end{tabular}

Tabelle 3.4: Molenbrüche polycyclischer aromatischer Kohlenwasserstoffe, die bei der Pyrolyse von $2 \% \mathrm{n}$-Hexan bei $2050 \mathrm{~K}$ und 5,0 MPa entstanden sind.

Membran stammen. Deshalb war die Wiederfindungsquote stets etwas kleiner als $100 \%$.

\subsection{Rußpartikelstruktur}

\subsubsection{Spezifische Oberfläche von Ruß}

Von Tesner [23] wurden die spezifischen Oberflächen $(A / m)$ von Ruß durch StickstoffAdsorption bei der Temperatur von flüssigem Stickstoff bestimmt. Die Proben stammen aus Benzol- und Hexanpyrolysen, die bei einer konstanten Kohlenstoffkonzentration von ca. $35 \mathrm{~mol} / \mathrm{m}^{3}$ und im Temperaturbereich zwischen 1800 und $2200 \mathrm{~K}$ ausgeführt wurden. Die spezifische Oberfläche dieser Rußproben beträgt unabhängig vom eingesetzten Kohlenwasserstoff $110 \mathrm{~m}^{2} / \mathrm{g} \pm 10 \%$. Unter der Annahme sphärischer Rußpartikel mit monomolekularer Stickstoff-Belegung der Rußoberfläche berechnet sich der Partikeldurchmesser zu $30 \mathrm{~nm}$.

$$
d=\frac{6}{\rho_{\mathrm{Ru} \beta} \cdot(A / m)}=30 \mathrm{~nm}
$$

\subsubsection{Elektronenmikroskopie}

Für die elektronenmikroskopische Untersuchung von Ruß wurde bei Temperaturen von ca. $2000 \mathrm{~K}$ n-Hexan und Benzol pyrolysiert. In den Benzolpyrolysen wurde bei einem Druck von $5 \mathrm{MPa}$ der Benzolanteil um zwei Größenordnungen von 200 bis 20000 ppm variiert, während in den Hexanpyrolysen bei Drücken von 5 und $10 \mathrm{MPa}$ die Kohlenstoffdichte konstant gehalten wurde. Es zeigte sich, daß alle Rußpartikel annähernd Kugelgestalt haben. Die Rußpartikel lagen zumeist in losen ketten- und haufenförmigen Agglomeraten vor. Die Größe und die Rußpartikeldichte der Agglomerate wurde nicht weiter ausgewertet, weil vermutlich schon vor der Probenentnahme die primäre Struktur der Agglomerate in den schnellen Gasströmungen im Stoßrohr verändert wurde. Die Partikel wurden auf den stark vergrößerten Fotografien ausgemessen. Von jeder Probe wurden, je nach Qualität der Fotografien, die Durchmesser von fünfzig- bis zweihundert Rußpartikel bestimmt. Die Durchmesser variierten dabei von 10 bis $200 \mathrm{~mm}$ und wurden in 19 Größenklassen von $5 \mathrm{~mm}$ Breite unterteilt. Daraus lassen sich, je nach Gesamtvergrößerung, 


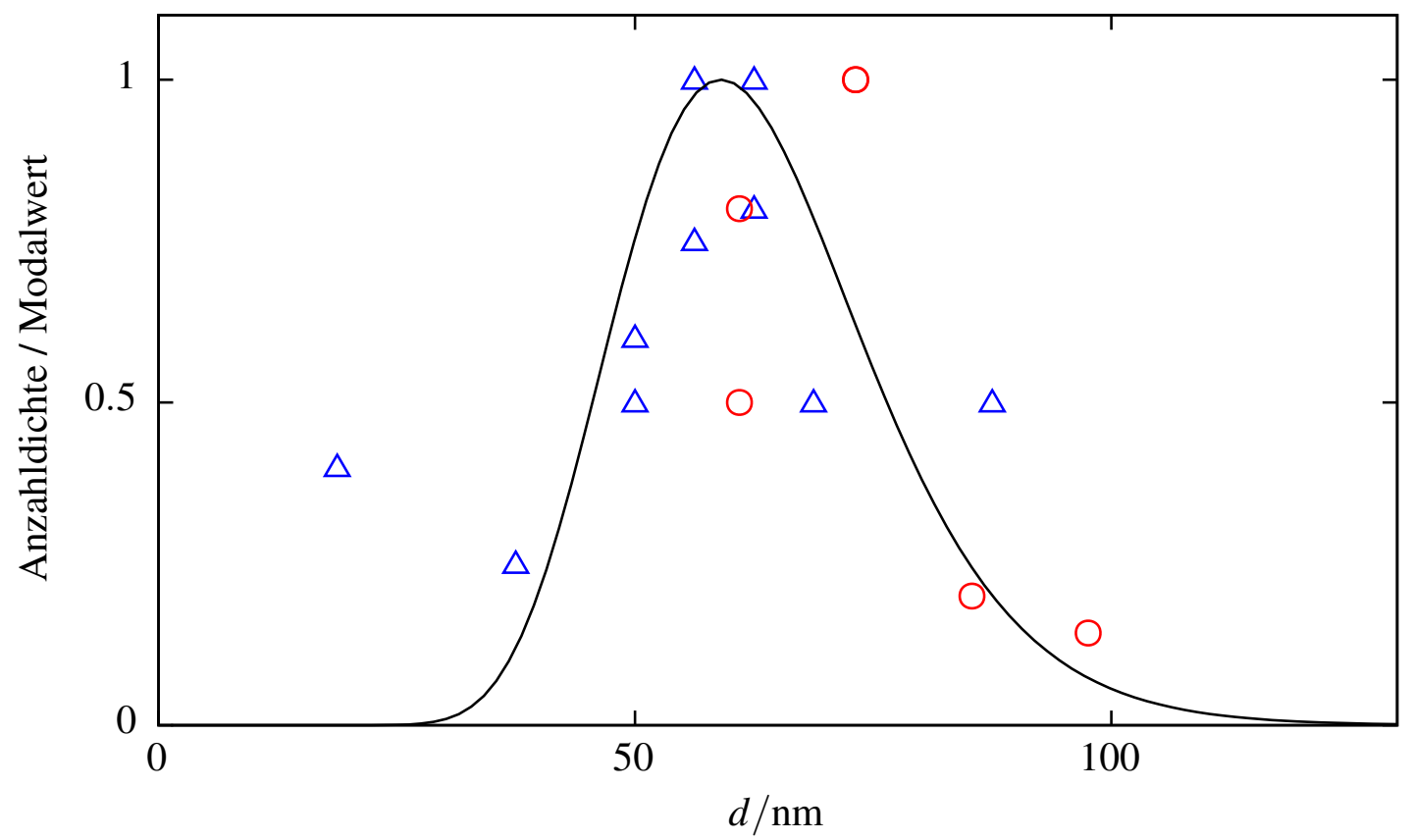

Abbildung 3.48: Rußpartikeldurchmesser aus der Pyrolyse von $2 \%$ n-Hexan bei $T$ $=2150 \mathrm{~K}$ und $P=4,8 \mathrm{MPa}$. Zur Untersuchung wurde ein REM verwendet. $\triangle: 80000$ fache und $\circ$ : 160000 -fache Vergrößerung. $d_{g}=62 \mathrm{~nm}$ und $\sigma_{g}=0,22$.

Größenklassen von 6,3 und $12 \mathrm{~nm}$ für die Rasterelektronenmikroskop-Aufnahmen und 1,1 und 2,5 nm für die Transmissionselektronenmikroskop-Aufnahmen berechnen. Zur Beschreibung der Durchmesserverteilung wurden die Meßergebnisse in Histogrammen dargestellt. Die Abbildungen (3.48) und (3.49) zeigen stellvertretend die Ergebnisse aus je einer charakteristischen REM- und TEM-Untersuchung. Dabei ist zu beachten, daß die Partikelradien, die mit dem Rasterelektronenmikroskop bestimmt wurden, nicht um die ca. $15 \mathrm{~nm}$ dünne Sputter-Goldschicht korrigiert wurden. Die Durchmesser der Rußpartikel einer Probe sind monomodal größenverteilt und lassen sich mit einer logarithmischen normalen Verteilungsfunktion in Gleichung (3.15) gut beschreiben. Das bedeutet, daß der natürliche Logarithmus der Partikeldurchmesser $\ln (d)$ einer Gauß-Verteilung folgt. Im Anhang sind die wichtigsten Eigenschaften dieser Funktion angegeben.

$$
P(d)=\int_{a}^{b} \frac{1}{\sqrt{2 \pi} \sigma_{g}} \cdot \exp \left[-\frac{\ln ^{2}\left(d / d_{g}\right)}{2 \sigma_{g}^{2}}\right] \mathrm{d} \ln (d)
$$

Die Parameter der Verteilungsfunktionen, der geometrische Durchmesser $d_{g}$ und die geometrische Standardabweichung $\sigma_{g}$ sind in Tabelle (3.5) angegeben. Aus den TEMUntersuchungen ergeben sich unabhängig von dem Kohlenwasserstoff, der Kohlenstoffdichte und der Temperatur ein mittlerer geometrischer Durchmesser von $20 \mathrm{~nm}$ und eine mittlere geometrische Standardabweichung von 0,20. Die REM-Ergebnissen zeigen die gleiche Standardabweichung aber einen deutlich größerern geometrischen Durchmesser von $74 \mathrm{~nm}$. Nach der Abzug der $15 \mathrm{~nm}$ dünnen Goldschicht von den Partikelradien verringert sich der mittlere Durchmesser auf $45 \mathrm{~nm}$. Gleichzeitig vergrößert sich die Standardabweichung auf 0,33. Im Vergleich zu den TEM-Ergebnissen sind die Durchmesser, die mit dem REM bestimmt wurden, deutlich größer. Dieser Unterschied findet sich auch 


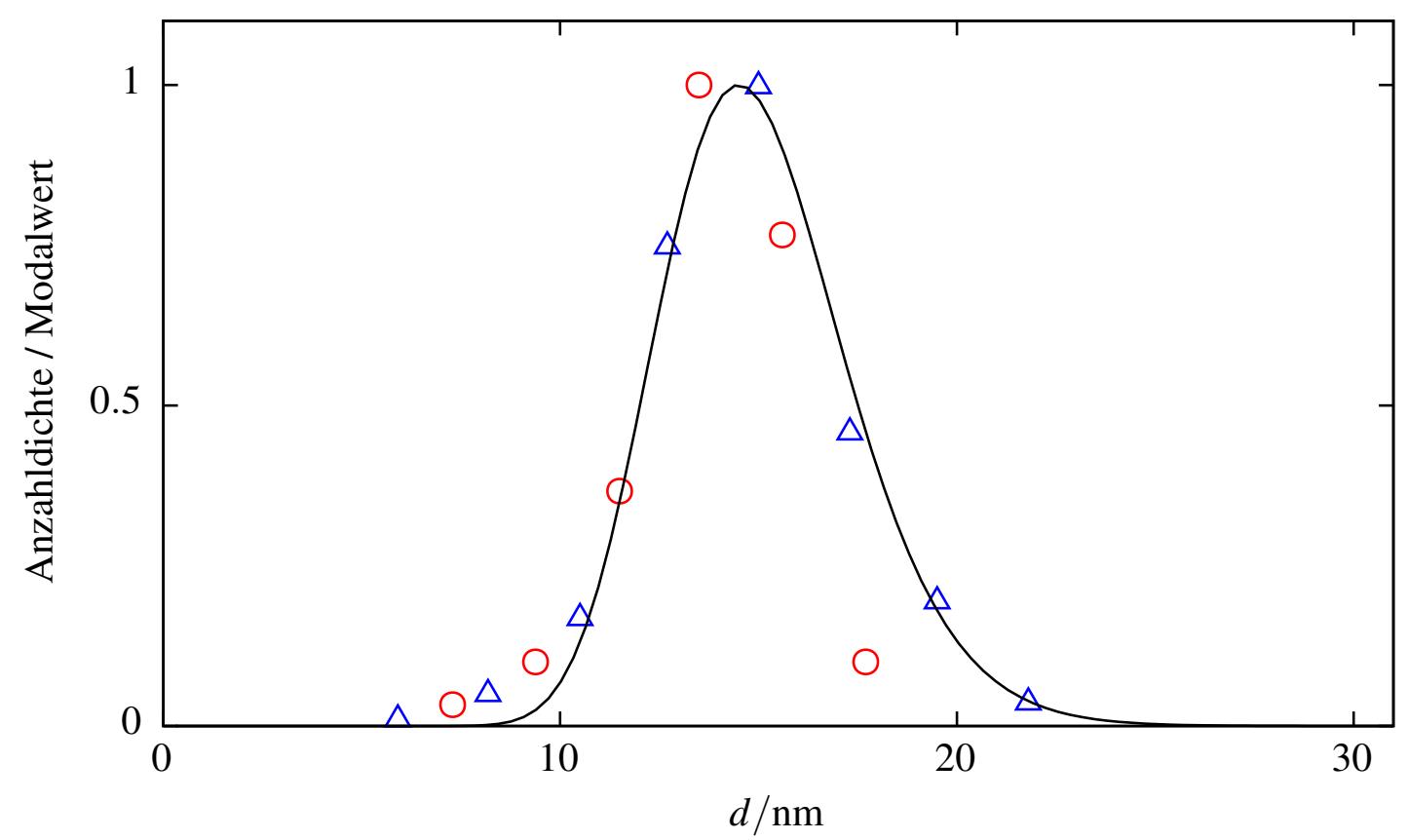

Abbildung 3.49: Rußpartikeldurchmesser aus der Pyrolyse von $2000 \mathrm{ppm}$ Benzol bei $T$ $=2020 \mathrm{~K}$ und $P=4,8 \mathrm{MPa}$. Zur Untersuchung wurde ein TEM verwendet. $\circ: 220000$ fache und $\triangle:$ : 480000-fache Vergrößerung. $d_{g}=15 \mathrm{~nm}$ und $\sigma_{g}=0,16$.

bei Rußproben, die aus ein und demselben Experiment stammen (siehe Zeile 3 und 5 in Tabelle (3.5)). Die Ursache der unterschiedlich bestimmten Durchmesser lag nicht an den verwendeten Apparaturen, da die Elektronenmikroskope im Institut für Metallphysik regelmäßig gewartet und geeicht wurden [24].

\begin{tabular}{rcccccc}
\hline Edukt & $T / \mathrm{K}$ & $P / \mathrm{MPa}$ & {$[C] / \frac{\mathrm{mol}}{\mathrm{m}^{3}}$} & ElMi & $d_{g} / \mathrm{nm}$ & $\sigma_{g}$ \\
\hline Benzol & 1900 & 5,1 & 0,39 & TEM & 20 & 0,20 \\
Benzol & 2020 & 4,8 & 3,4 & TEM & 16 & 0,16 \\
Benzol & 2110 & 4,4 & 30 & TEM & 17 & 0,18 \\
n-Hexan & 2000 & 4,9 & 35 & TEM & 25 & 0,25 \\
\hline Benzol & 2110 & 4,4 & 30 & REM & $86(56)$ & $0,18(0,30)$ \\
n-Hexan & 2150 & 4,8 & 32 & REM & $62(33)$ & $0,22(0,35)$ \\
n-Hexan & 1990 & 10,3 & 37 & REM & $75(45)$ & $0,20(0,34)$ \\
\hline
\end{tabular}

Tabelle 3.5: Die Rußpartikeldurchmesser wurden mit einer logarithmisch normalen Verteilungsfunktion approximiert. Deren Parametern sind der geometrische Durchmesser $d_{g}$ und die geometrische Standardabweichung $\sigma_{g}$. Die eingeklammerten Werte wurden nach Subtraktion der Goldschicht erhalten. 


\section{Kapitel 4}

\section{Diskussion}

In diesem Kapitel werden die Meßergebnisse zusammengefaßt, gedeutet und im Vergleich zu Meßwerten diskutiert, die aus der Literatur entnommen wurden.

\subsection{Rußpartikeleigenschaften}

\subsubsection{Durchmesser}

Die Durchmesser der Rußpartikel wurden sowohl durch BET-Stickstoff-Adsorption als auch durch Auswertung elektronenmikroskopischer Aufnahmen bestimmt. Die Rußproben stammen aus Pyrolysen, in denen Temperatur, Druck und die Kohlenstoffkonzentration in weiten Grenzen variiert wurden. Von diesen Parametern unabhängig wurde für alle Rußproben ein mittlerer Durchmesser der Partikel im Bereich von 20 bis $30 \mathrm{~nm}$ gemessen. Dabei wurden mit dem REM stets größere Durchmesser bestimmt als mit dem TEM. Die Ursache für diese Unterschiede liegt in den verschiedenen Techniken der Vorbereitung und Untersuchung der Proben. Das Verhältnis des Durchmessers zur Wellenlänge des Laserlichtes $(632,8 \mathrm{~nm})$ ist kleiner als 0,05. Damit war eine wichtige Bedingung $\left(d_{\text {Rußpartikel }} / \lambda_{\text {Laserlicht }}\right)<0,1$ zur Anwendung der Rayleigh-Näherung in allen Experimenten erfüllt.

Der Rußpartikeldurchmesser von $30 \mathrm{~nm}$ stellt einen oberen Grenzwert dar. Der gleiche maximale Durchmesser wurde in vorgemischten Unterdruck- [25] und Hochdruckflammen [26] gefunden. Die Verteilungsbreite der Durchmesser verringerte sich mit zunehmendem Druck bzw. zunehmender Kohlenstoffdichte von $\sigma_{g} \approx 0,3$ auf $\leq 0,2$. Mit $\sigma_{g}$ $\leq 0,2$ ist das Kriterium einer engen Verteilung erfüllt [13]. In diesen Fall ist der Durchmesser $d_{36}$, siehe Gleichung (5.8), der aus elastischen Streulichtexperimenten bestimmt werden könnte, nur noch knapp $20 \%$ größer als der geometrische Durchmesser $d_{g}$. Durchmesserverteilungen mit dieser kleinen Verteilungsbreite werden auch mit symmetrischen Verteilungsfunktionen gut beschrieben. In den Abbildungen (3.48) und (3.49) können die Häufigkeitsverteilungen der Rußpartikeldurchmesser mit Gaußnormalverteilungen angepaßt werden. Dabei beträgt die auf den häufigsten Durchmesser $d_{\text {Modal }}$ normierte Standardabweichung $\left(\sigma / d_{\text {Modal }}\right)=0,2 \pm 10 \%$.

Auf den Fotografien war zu erkennen, daß die Rußpartikel in losen Agglomeraten zusammengelagert sind. Gegebenenfalls waren die Agglomerate in der Gasphase größer, zerfielen jedoch möglicherweise während der Probenentnahme. Diese Größenänderung der Agglomerate nahm keinen Einfluß auf die Durchmesser der primären Rußpartikel.

In Flammenexperimenten, in denen Licht an Rußpartikel elastisch gestreut wurde, 
wurden Durchmesser von 1 bis $100 \mathrm{~nm}$ gemessen [27, 28]. In Normaldruckflammen wurde eine Zunahme der Enddurchmesser $d_{\infty}$ bei konstanter Endpartikelanzahldichte $N_{\infty}=10^{15} / \mathrm{m}^{3}$ mit der Überschuß-Kohlenstoffdichte gefunden. In in Hochdruck-Ethylenflammen dagegen nimmt die Endpartikelanzahldichte mit der Überschuß-Kohlenstoffdichte stark zu. Die großen Rußpartikeldurchmesser mit $d>50 \mathrm{~nm}$ können mit der Meßmethode erklärt werden, da die Strukturen mit großem Durchmesser besonders hohe Streulichtintensitäten verursachen. Der Streuquerschnitt von sphärischen Teilchen ist proportional zur sechsten Potenz des Partikeldurchmesser [13]. Schon eine kleine Konzentration von großen Agglomeraten täuscht einen höheren Partikeldurchmesser vor. Zudem muß bei einer Verteilungsbreite von $\sigma_{g}=0,3$ der Durchmesser um $50 \%$ nach unten korrigiert werden, siehe Gleichung (5.8).

\subsubsection{Rußpartikelanzahldichte}

Die Partikelanzahldichte $N$ wird aus dem Verhältnis von Rußvolumenbruch und dem mittleren Rußpartikelvolumen $(\bar{v})$ berechnet, siehe Gleichung (4.1).

$$
N=f_{v} / \bar{v} \quad \text { mit: } \quad \bar{v}=\text { mittleres Rußpartikelvolumen }
$$

Am Anfang der Rußbildung wird die Partikelanzahldichte $N_{0}$ aus der Rußvolumenbruch-Nachweisgrenze $\left(f_{v o}=10^{-7}\right)$ und dem mittleren Rußpartikelvolumen $\bar{v}_{0}$ der kleinsten Rußpartikel abgeschätzt. Das mittlere Rußpartikelvolumen wird aus dem Rußpartikelradius $r_{0}$ berechnet, der von Homann [29] in vorgemischten Unterdruckflammen massenspektrometrisch bestimmt wurde: $r_{o} \approx 1 \mathrm{~nm}$ und $\bar{v}_{0} \approx 5 \cdot 10^{-27} \mathrm{~m}^{3}$. Damit egibt sich für $N_{0}=2 \cdot 10^{19} / \mathrm{m}^{3}$.

Bei einem mittleren Durchmesser am Ende der Rußbildung von $d_{\infty}=25 \mathrm{~nm}$ ist $\bar{v}=10^{-23} \mathrm{~m}^{3}$. In $5 \mathrm{MPa}$-Benzolpyrolysen mit einer Kohlenstoffkonzentration von $0,4 \mathrm{~mol} / \mathrm{m}^{3}$ wurde ein $f_{v \infty}$ von $5 \cdot 10^{-7}$ gefunden. Mit Gleichung (4.1) berechnet sich die Partikelanzahldichte zu $N_{\infty}=5 \cdot 10^{16} / \mathrm{m}^{3}$.

In Experimenten mit Kohlenstoffkonzentrationen die um zwei Größenordnungen höher waren, wurden ähnliche Durchmesser und höhere Rußvolumenbrüche von ca. $10^{-4}$ bzw. Partikelanzahldichten von $10^{19} / \mathrm{m}^{3}$ gefunden.

Für vorgemischte Normaldruck-Ethylen-Flammen wurden Partikelanzahldichten von $10^{15}$ bis $10^{16} 1 / \mathrm{m}^{3}$ erhalten [18], während in $7 \mathrm{MPa}$-Ethylen-Luft-Flammen Partikelanzahldichten von $10^{18} / \mathrm{m}^{3}$ gemessen wurden [30]. Mit der Kenntnis der Durchmesser und der Anzahldichte von Rußpartikel wird im folgenden Abschnitt die Koagulation der Partikel betrachtet.

\subsubsection{Koagulation}

Treffen zwei Rußpartikel aufeinander, können sie aneinander haften bleiben oder dissoziieren. In Normaldruckflammen ist die Haftwahrscheinlichkeit nahe bei eins [18], während in fetten Hochdruckflammen die Dissoziation zunehmend überwiegt [31]. Dieser Prozeß kann mit der Smoluchowski Gleichung (4.2) beschrieben werden [32], mit der die charakteristische Halbwertszeit der Koagulation $\left(\tau_{\text {koag }}\right)$ gegen Ende der Rußbildung theoretisch berechnet werden kann.

$$
\frac{\mathrm{d} N}{\mathrm{~d} t}=-k_{\mathrm{koag}} \cdot[N]^{2}
$$




$$
\tau_{\text {koag }}=\left(k_{\text {koag,theo }} \cdot N_{\infty}\right)^{-1}
$$

Die Koagulationsgeschwindigkeitskonstante $\left(k_{\text {koag,theo }}\right)$ wird mit der Theorie über Aerosole berechnet [32]. Die KNUDSEN-Zahl Kn, als wichtigste Kennzahl der Aerosole, ist das Verhältnis der freien Weglänge des Gases $\lambda_{\text {Gas }}$ und des Partikelradius $r$.

$$
K n=\frac{\lambda_{\mathrm{Gas}}}{d_{g} / 2}
$$

In dieser Arbeit waren die KNUDSEN-Zahl zum Ende der Rußbildung im Bereich zwischen 0,1 und 1. Das ist der sogenannte ,slip flow“ Bereich, der sich zwischen Kontinuumsgebiet $(K n \rightarrow 0)$ und Übergangsbereich $(1 \leq K n \leq 10)$ befindet. In diesem Bereich beträgt die theoretische Koagulationsgeschwindigkeitskonstante $k_{\mathrm{koag}}=5 \cdot 10^{-16} \mathrm{~m}^{3} / \mathrm{s}$. Für Benzolexperimente mit geringer Kohlenstoffdichte $\left(0,4 \mathrm{~mol} / \mathrm{m}^{3}\right)$ ergibt sich eine Halbwertszeit $\tau_{\text {koag }}$ zum Ende der Rußbildung von $40 \mathrm{~ms}$. Das ist eine zu lange Zeitkonstante um die Koagulation in Stoßwellenexperimenten weiter zu verfolgen. Dagegen ist die Zeitkonstante in Benzolexperimenten hoher Kohlenstoffdichte $\left(40 \mathrm{~mol} / \mathrm{m}^{3}\right)$ nur 0,2 ms kurz, also innerhalb der Beobachtungszeit von Stoßwellenexperimenten. In diesen Experimenten hätte man deutlich größere Rußpartikel erwarten können. Das ist ein Hinweis darauf, daß das ,,meet and stick“-Koagulationsmodell, mit der Annahme hoher Haftwahrscheinlichkeit $(\approx 1)$, für hohe Kohlenstoffdichten nicht mehr gültig sein kann. Für diesen Fall lassen sich der Zeitprofile der Partikelanzahldichten $N(t)$ mit der Smoluchowski Gleichung beschreiben, die um einen Dissoziationsterm $\left(k_{\text {diss }} \cdot[N]\right)$ erweitert wurde [27].

\subsection{Einfluß der Lichtwellenlänge auf die detektierte Rußausbeute}

$\mathrm{Zu}$ Beginn der Rußbildung ist die Rußausbeute, die mit einem HeNe-Laser $(632,8 \mathrm{~nm})$ bestimmt wurde kleiner als die mit einem Argonionen-Laser $(488 \mathrm{~nm})$ gemessene Rußausbeute [1]. Den Abbildungen (3.3) und (3.15) ist zu entnehmen, daß sich dieser Effekt zu noch höheren Wellenlängen fortsetzt. Die mit der Laserdiode $(1064 \mathrm{~nm})$ bestimmte Rußausbeute war in dieser Phase der Rußbildung bis zu sechsfach geringer und die Induktionszeiten waren bis zu zweimal länger. Der Einfluß der Lichtwellenlänge auf die Rußausbeute kann mit zwei Beobachtungen aus Flammenexperimenten erklärt werden. Von Schulz [33] wurde gezeigt, daß die elektrische Leitfähigkeit von Ruß, der aus fetten Niederdruckflammen aufgefangen wurde, mit zunehmender Reaktionszeit stark abnimmt. Da die elektrische Leitfähigkeit und der Brechungsindex korrelieren, wäre bei der Auswertung von Intensitäts-Zeitprofilen die Zeitabhängigkeit des Brechungsindex zu berücksichtigen.

In der Frühphase der Rußbildung entstehen vergleichbare Mengen polycyclischer Aromaten [16]. Die Hochtemperaturspektren dieser Spezies sind noch nicht hinreichend bekannt. Es ist aber anzunehmen, daß die PAH nicht nur im UV sondern auch im VIS, insbesondere bei $623,8 \mathrm{~nm}$, Licht absorbieren. Unter der Annahme, daß VIS-absorbierende Zwischenprodukte entstehen, wurden mit einem Reaktionsgeschwindigkeitsansatz erster Ordnung (siehe Gleichung (4.5)) zwei Geschwindigkeitskonstanten, für die Produktion $k_{a}$ und den Verbrauch $k_{b}$ der Zwischenprodukte in einer Methanpyrolyse bei $2150 \mathrm{~K}$, numerisch berechnet: $k_{a}=20000 / \mathrm{s}$ und $k_{b}=15000 / \mathrm{s}$. Die gestrichelte Kurve in Abbildung (3.3) zeigt das Ergebnis dieser Anpassung. 


$$
\text { Edukt } \stackrel{k_{a}}{\longrightarrow} \text { Zwischenprodukte } \stackrel{k_{b}}{\longrightarrow} \text { Produkte }
$$

Unmittelbar nach der Induktionszeit der Rußbildung begann die schnelle Abnahme der Zwischenprodukte und war am Wendepunkt des Rußbildungzeitprofils nahezu beendet. Vermutlich haben die Zwischenprodukte zu einem geringen Anteil zum Rußmassenwachstum beigetragen. Neuere Absorptionsmessungen mit dem infraroten Licht eines $\mathrm{CO}_{2}$-Laser $(\lambda=10,6 \mu \mathrm{m}$ zeigten, daß sich die Induktionszeiten nur noch unwesentlich mit zunehmender Wellenlänge verlängerten [34].

\subsection{Einfaches Modell zur Temperaturabhängigkeit der Rußausbeute}

In dieser Arbeit wurde die Temperaturabhängigkeit der Rußausbeute für alle untersuchten Kohlenwasserstoffe mit einem einfachen Drei-Spezies-Modell approximiert, siehe Gleichung (4.6). Das Edukt befindet sich mit den Zwischenprodukten und dem Ruß im gekoppelten Gleichgewicht:

$$
\text { Edukt } \underset{k_{21}}{\stackrel{k_{12}}{\rightleftharpoons}} \text { Zwischenprodukte } \underset{k_{32}}{\stackrel{k_{23}}{\rightleftharpoons}} \text { Ruß }
$$

Die analytische Lösung der zeitlichen Änderung der Rußmenge $\left(\mathrm{d}[C]_{\mathrm{Ruß}} / \mathrm{d} t\right)$ ist bekannt [35]. Unter der Annahme des linearen Arrhenius-Verhaltens für die vier Reaktionsgeschwindigkeiten $\left(k_{12}, k_{21}, k_{23}\right.$ und $\left.k_{32}\right)$ wurde die Temperaturabhängigkeit der Rußausbeute $R A(T)$ für konstanten Druck $\left(\Delta P_{\text {Gesamt }}=0\right)$ und konstanter Kohlenstoffdichte $\left(\Delta[C]_{\text {Gesamt }}=0\right)$ berechnet, siehe Gleichung (4.7). In diesem Modell ist die Rußausbeute nur von den Gleichgewichtskonstanten $\left(K_{12}\right)$ und $\left(K_{23}\right)$ abhängig. In Gleichung (4.8) werden die Gleichgewichtskonstanten aus dem Verhältnis der Reaktionsgeschwindigkeiten für die Rück- und Hinreaktion berechnet. Diese Definition der Gleichgewichtskonstanten entspricht dem Kehrwert der konventionellen Methode (Quotient aus Hin- und Rückreaktion). Mit der hier verwendeten Definition läßt sich die Ableitung der Rußausbeute nach der inversen Temperatur $\mathrm{d}(R A) / \mathrm{d}(1 / T)$ in Gleichung (4.7) erheblich einfacher bilden.

$$
\begin{gathered}
R A(T)=\left[1+K_{23}(T) \cdot\left(1+K_{12}(T)\right)\right]^{-1} \\
K_{12}(T)=\frac{k_{21}}{k_{12}} \quad \text { und } \quad K_{23}(T)=\frac{k_{32}}{k_{23}}
\end{gathered}
$$

Die Gleichgewichtskonstanten in Gleichungen (4.13) und (4.14) hängen von vier Parametern ab. Diese Parameter sind die beiden Reaktionsenthalpien $\Delta_{R} H_{12}$, und $\Delta_{R} H_{23}$, die Höhe der maximalen Rußausbeute $R A_{\max }$ und die zugehörige Temperatur $T_{\max }^{R A}{ }^{1}$.

${ }^{1}$ Zunächst wird die Änderung der Rußausbeute $R A$ mit der inversen Temperatur $1 / T$ berechnet.

$$
\frac{\mathrm{d}(R A)}{\mathrm{d}(1 / T)}=-(R A)^{2} \cdot\left[\left(1+K_{12}\right) \cdot \frac{\mathrm{d} K_{23}}{\mathrm{~d}(1 / T)}+K_{23} \cdot \frac{\mathrm{d} K_{12}}{\mathrm{~d}(1 / T)}\right]
$$

Da für die vier Geschwindigkeitskonstanten lineares Arrhenius-Verhalten angenommen wurde, läßt sich die Temperaturabhängigkeit der Gleichgewichtskonstanten nach van't Hoff beschreiben.

$$
K_{12}=A_{12} \cdot \exp \left(-\Delta_{R} H_{12} / R T\right) \quad \text { und } \quad K_{23}=A_{23} \cdot \exp \left(-\Delta_{R} H_{23} / R T\right)
$$




$$
\begin{aligned}
& K_{12}(T)=-\exp \left[\frac{\Delta_{R} H_{12}}{R} \cdot\left(\frac{1}{T}-\frac{1}{T_{\max }^{R A}}\right)\right]\left(1+\frac{\Delta_{R} H_{12}}{\Delta_{R} H_{23}}\right)^{-1} \\
& K_{23}(T)=-\exp \left[\frac{\Delta_{R} H_{23}}{R} \cdot\left(\frac{1}{T}-\frac{1}{T_{\max }^{R A}}\right)\right]\left(1+\frac{\Delta_{R} H_{23}}{\Delta_{R} H_{12}}\right)\left(1-\frac{1}{R A_{\max }}\right)
\end{aligned}
$$

Mit einer numerischen Anpassung wurden die berechneten Rußausbeuten den Meßwerten angepaßt. Dabei konnten alle Experimente schon mit diesem relativ einfachen Modell gut beschrieben werden. Physikalisch sinnvolle Lösungen ergeben sich nur mit gegenläufigen Temperaturabhängigkeiten der Gleichgewichtskonstanten. Außerdem muß der Betrag der Reaktionsenthalpie $\Delta_{R} H_{12}$ größer sein als der Betrag von $\Delta_{R} H_{23}$. Die Ergebnisse in Tabelle (3.3) zeigen, daß die erste Reaktion um $790 \mathrm{~kJ} / \mathrm{mol}$ für Aromaten und $1080 \mathrm{~kJ} / \mathrm{mol}$ für Nicht-Aromaten exotherm ist. Die Reaktionsenthalpie $\Delta_{R} H_{12}$ wird der Bildung von Wachstumsspezies zugeordnet. Es wird angenommen, daß in der Pyrolyse von Nicht-Aromaten die Polyacetylene $\mathrm{C}_{2 n} \mathrm{H}_{2}$ mit $1 \leq n \leq 4$ wichtige Wachstumsspezies sind. Weiterhin wird angenommen, daß die Bildungsenthalpien von Ruß und Graphit bei hohen Temperaturen nicht sehr verschieden voneinander sind, $\Delta_{f} H_{\text {Graphit }}^{2000 K}=35 \mathrm{~kJ} / \mathrm{mol}$. Die Bildungsenthalpie des Polyacetylen $\mathrm{C}_{6} \mathrm{H}_{2}$ beträgt $971 \mathrm{~kJ} / \mathrm{mol}$ bei $2000 \mathrm{~K}$. Dieser Wert unterscheidet sich nur wenig von $\Delta_{R} H_{12}$. In der Pyrolyse von Aromaten wurde ein kleinerer Wert für $\Delta_{R} H_{12}$ ermittelt, weil neben Acetylen auch Aromaten als Wachstumsspezies auftreten. Die Bildungsenthalpie von Benzol ist $439 \mathrm{~kJ} / \mathrm{mol}$ bei $2000 \mathrm{~K}$ und damit nur ca. halb so groß wie die Bildungsenthalpie von $\mathrm{C}_{6} \mathrm{H}_{2}$. Mit diesem Modell lassen sich nicht nur die Rußausbeuten am Ende der Pyrolyse berechnen sondern auch vollständige Zeitprofile der Rußausbeute $R A(t)$ simulieren. Dazu ist ein fünfter Parameter notwendig. Dieser Parameter ist der Zeitpunkt $t_{0}$, an dem der erste Ruß auftritt. $t_{0}$ ist stets etwas kleiner als die Induktionszeit $\tau$.

\subsection{Gasförmige Pyrolyseprodukte}

Die im Rahmen dieser Arbeit analysierten Gase können in zwei Gruppen aufgeteilt werden. Die erste Gruppe enthält die Hauptprodukte, Acetylen und Wasserstoff, mit Ausbeuten von durchschnittlich über $10 \%$. Die Wasserstoffausbeute nimmt mit steigender Temperatur stetig zu, während für Acetylen Minima in der Temperaturabhängigkeit der Ausbeute gefunden wurden. Zu der zweiten Gruppe werden Methan, Ethylen, Ethan und die $\mathrm{C}_{3}$ Kohlenwasserstoffe gezählt, deren Ausbeuten im Durchschnitt kleiner sind als $10 \%$. Im Unterschied zu den Spezies aus der ersten Gruppe nimmt die Ausbeute unabhängig von der Kohlenstoffdichte und dem Brennstofftyp mit steigender Temperatur stetig ab.

Zur Erklärung der unterschiedlichen Höhe und Temperaturabhängigkeit der Ausbeuten wird das Reaktionssystem thermodynamisch betrachtet. In Abbildung (4.1) sind die Bildungs-Gibbsenthalpien $\Delta_{f} G(1 \mathrm{~atm})$ von Methan, Acetylen, Ethylen und Ethylen in

Die Vorfaktoren $A_{12}$ und $A_{23}$ werden mit Hilfe der Maximalbedingung $d(R A) / d(1 / T)=0$ berechnet.

$$
\begin{aligned}
& A_{12}=-\left[1+\frac{\Delta_{R} H_{12}}{\Delta_{R} H_{23}} \cdot \exp \left(\frac{\Delta_{R} H_{12}}{R T_{\max }^{R A}}\right)\right]^{-1} \\
& A_{23}=\left(1+\frac{\Delta_{R} H_{23}}{\Delta_{R} H_{12}}\right) \cdot\left(\frac{1}{R A_{\max }}-1\right) \cdot \exp \left(\frac{\Delta_{R} H_{23}}{R T_{\max }^{R A}}\right)
\end{aligned}
$$




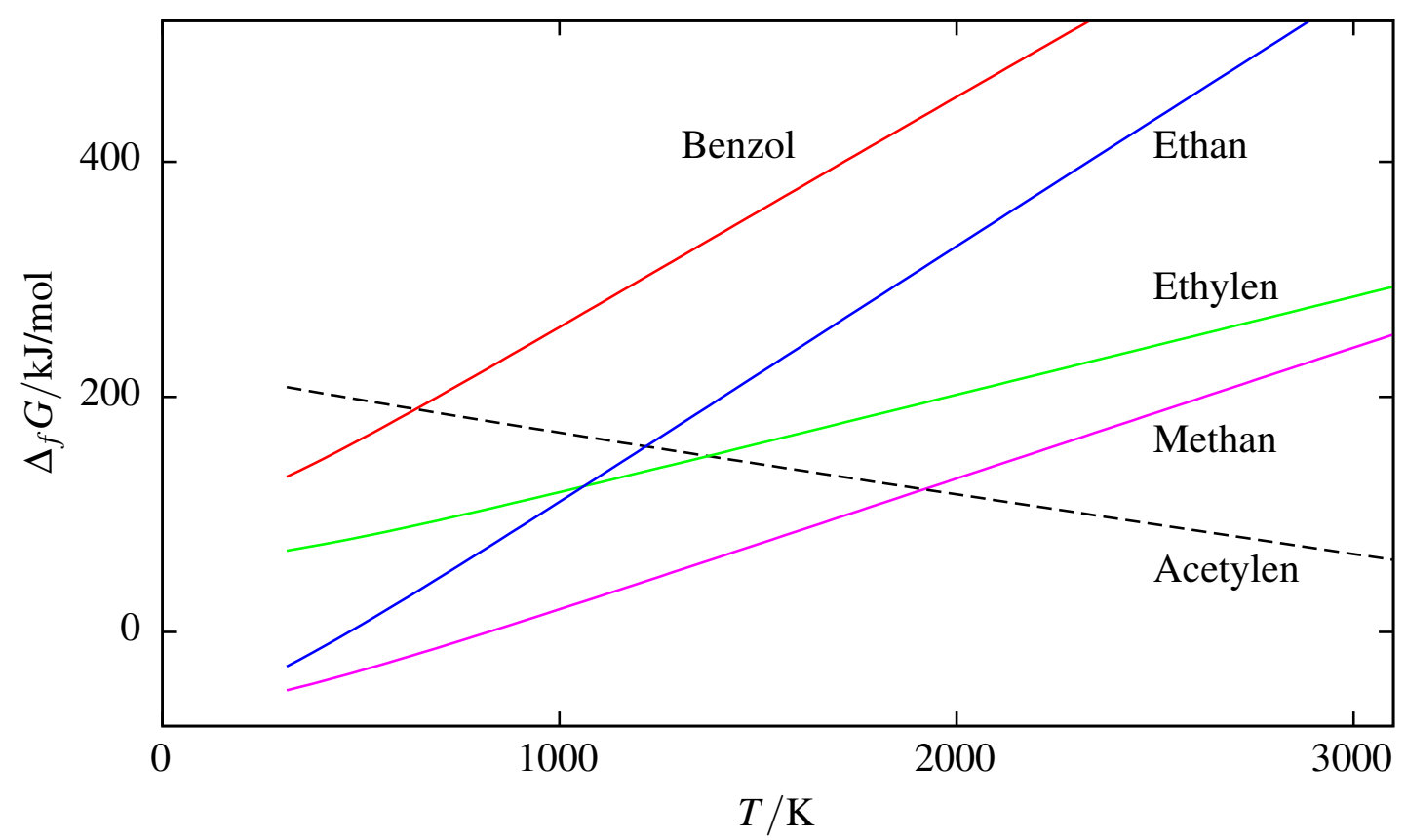

Abbildung 4.1: Bildungs-Gibbsenthalpien $\Delta_{f} G$ der wichtigsten Pyrolyseprodukte im Temperaturbereich von der Standardtemperatur $298 \mathrm{~K}$ bis $3000 \mathrm{~K}$.

dem für die Verbrennungsvorgänge relevanten Temperaturbereich $(T<3000 \mathrm{~K})$ aufgetragen. Zusätzlich ist als wichtiger Vertreter der Aromaten Benzol dargestellt. Die BildungsGibbsenthalpien wurden auf Grundlage der Daten aus den NASA-Polynomen [36] den JANAF-Tabellen [37] einer Thermodynamik-Datenbank [38] und der neuesten verfügbaren Sammlung thermodynamischer Daten [39] berechnet. Die Bildungs-Gibbsenthalpie der Elemente Kohlenstoff und Wasserstoff ist für alle Temperaturen und Drücke bekannterweise gleich Null, $\Delta_{f} G($ Elemente $)=0$. Von $900 \mathrm{~K}$ an aufwärts sind die BildungsGibbsenthalpien der Kohlenwasserstoffe der zweiten Gruppe größer Null und nehmen nahezu linear mit der Temperatur stark zu. Experimentell bestätigte sich diese thermodynamische Instabilität in den abnehmenden Ausbeuten der Spezies mit zunehmender Temperatur. Die Konzentrationen der $\mathrm{C}_{3}$ Kohlenwasserstoffe lagen unterhalb der Nachweisgrenze $(\approx 50 \mathrm{ppm})$. Das war wegen der hohen Bildungs-Gibbsenthalpien zu erwarten. Die einzige Ausnahme in der Temperaturabhängigkeit der Bildungs-Gibbsenthalpie ist Acetylen. Von $+209 \mathrm{~kJ} / \mathrm{mol}$ bei Standardtemperatur nimmt die Bildungs-Gibbsenthalpie stetig ab und wird bei $4350 \mathrm{~K}$ Null. Ab $1700 \mathrm{~K}$ ist Acetylen der Kohlenwasserstoff mit der kleinsten Bildungs-Gibbsenthalpie pro Mol Kohlenstoff. Sowohl die thermodynamischen Berechnungen als auch die Ergebnisse der Pyrolyseexperimente ergeben die gleichen Temperaturabhängigkeiten und die gleichen relativen Ausbeuten.

Für eine quantitative Betrachtung der Produktgaszusammensetzung muß der Druckeinfluß auf die Gleichgewichtskonstante $K_{P}$ und der Einfluß der Wasserstoffkonzentration berücksichtigt werden. Besonders empfindlich reagiert das Gleichgewicht zwischen Methan und Acetylen (Gleichung (4.15)) auf diese Einflüße. Wasserstoff geht mit der dritten Potenz in das Gleichgewicht ein und die Molzahländerung $\Delta n$ beträgt +2 . Aus den in Abbildung (4.1) dargestellten Bildungs-Gibbsenthalpien berechnet sich die Reaktionsenthalpie $\Delta_{R} H$ zu $406 \mathrm{~kJ} / \mathrm{mol}$. Die Gleichgewichtsreaktion zwischen Acetylen und Ethylen (Gleichung (4.16)) ist weniger stark von der Temperatur, dem Druck und der Wasserstoff- 


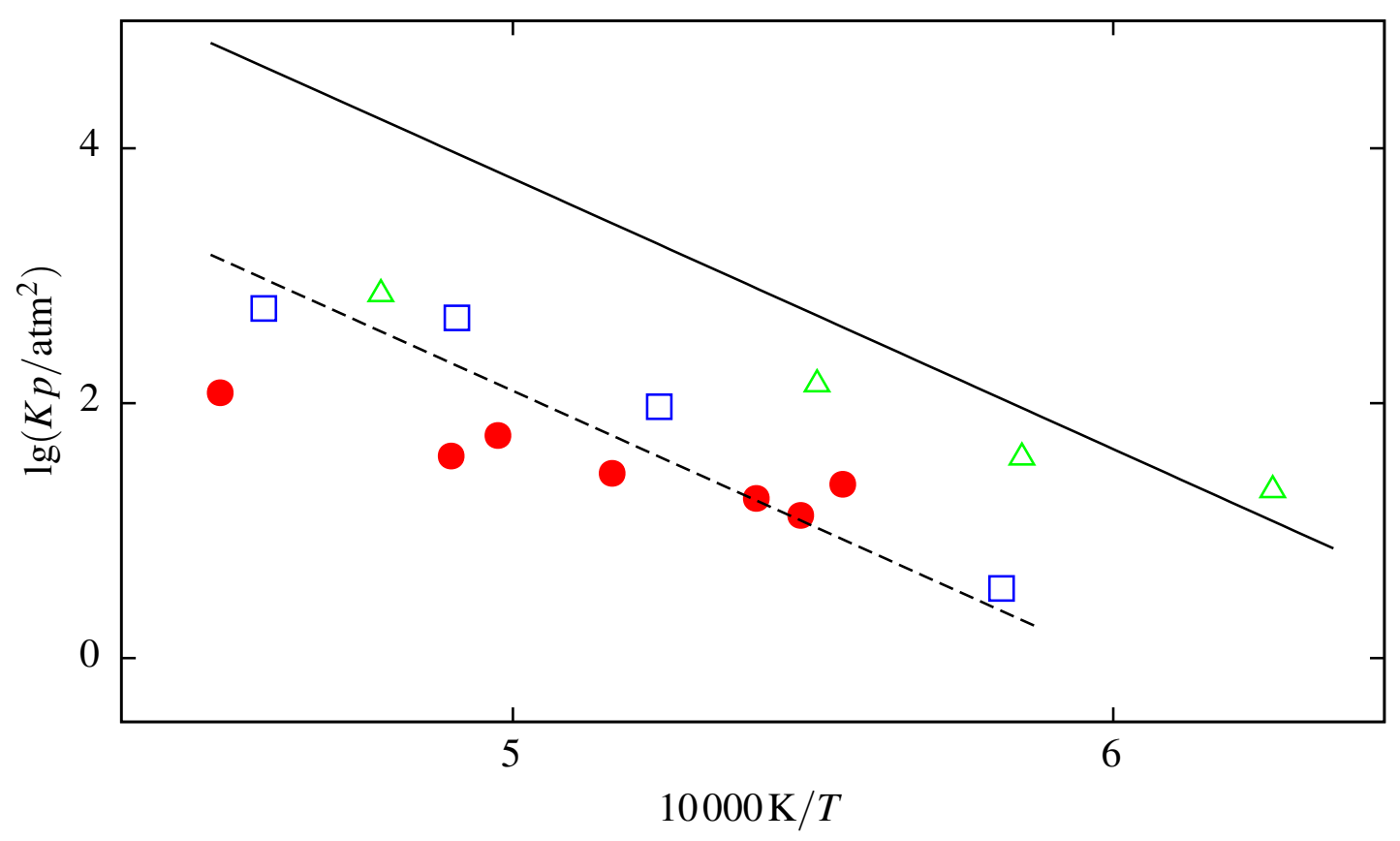

Abbildung 4.2: Berechnete Gleichgewichtskonstanten (durchgezogene Gerade) für die Reaktion $2 \mathrm{CH}_{4} \rightleftharpoons 3 \mathrm{H}_{2}+\mathrm{C}_{2} \mathrm{H}_{2}$, und experimentelle Werte aus $\bullet$ : Benzol-, $\triangle$ : Ethylenund $\square$ : Hexanpyrolysen bei $P=(5,0 \pm 0,5) \mathrm{MPa}$ und $[C]=(5 \pm 1) \mathrm{mol} / \mathrm{m}^{3}$.

konzentration abhängig.

$$
\begin{array}{rlr}
2 \mathrm{CH}_{4} \rightleftharpoons 3 \mathrm{H}_{2}+\mathrm{C}_{2} \mathrm{H}_{2} & K_{P}=\frac{P_{\mathrm{C}_{2} \mathrm{H}_{2}} \cdot P_{\mathrm{H}_{2}}^{3}}{P_{\mathrm{CH}_{4}}^{2}} & \Delta_{R} H=406 \mathrm{~kJ} / \mathrm{mol} \\
\mathrm{C}_{2} \mathrm{H}_{4} \rightleftharpoons \mathrm{H}_{2}+\mathrm{C}_{2} \mathrm{H}_{2} & K_{P}=\frac{P_{\mathrm{C}_{2} \mathrm{H}_{2}} \cdot P_{\mathrm{H}_{2}}}{P_{\mathrm{C}_{2} \mathrm{H}_{4}}} & \Delta_{R} H=186 \mathrm{~kJ} / \mathrm{mol}
\end{array}
$$

In den Abbildungen (4.2) und (4.3) werden die berechneten Gleichgewichtskonstanten $K_{P}$ der Reaktionen aus Gleichung (4.15) und (4.16) mit experimentellen Werten aus Benzol-, Ethylen- und Hexanpyrolysen verglichen. Daraus ergibt sich, daß die berechneten Gleichgewichtskonstanten $K_{P}$ (Thermodynamik) etwas größer sind als die experimentellen Werte $K_{P}$ (Experiment). Außerdem stimmen die Temperaturabhängigkeiten der berechneten Gleichgewichtskonstanten $\Delta_{R} H$ mit den Meßwerten gut überein. Daraus kann gefolgert werden, daß die partiellen Gleichgewichte zwischen Methan/Acetylen sowie Ethylen/Acetylen nach einer Pyrolysezeit von ca. 2 ms nahezu eingestellt sind.

In Abbildung (4.4) sind die Ruß- und Acetylenausbeuten in der Hexanpyrolyse $\left([C]=5,3 \mathrm{~mol} / \mathrm{m}^{3}\right)$ zusammen dargestellt. Die Temperaturabhängigkeiten der Ausbeuten verhalten sich gegenläufig. Das Minimum der Acetylenausbeute tritt bei der Temperatur auf, bei der die Rußausbeute ein Maximum hat. Dieses Verhalten findet sich auch in Ethylen- und Benzolpyrolysen wieder.

Die hohe Qualität der Gasausbeutemessungen zeigt sich in den Wasserstoffmassenbilanzen, in denen die Wiederfindungsquote stets rund $100 \%$ betrug. In den Experimenten, in denen die Rußausbeute optisch bestimmt werden konnte, wurden in den Kohlenstoffmassenbilanzen Wiederfindungsquoten von ca. $100 \%$ gefunden. Somit kann aus den Experimenten, in denen wegen der hohen Kohlenstoffdichten die Rußausbeuten optisch nicht betimmbar waren, die Gase jedoch analysiert wurden, die Rußausbeute abgeschätzt 


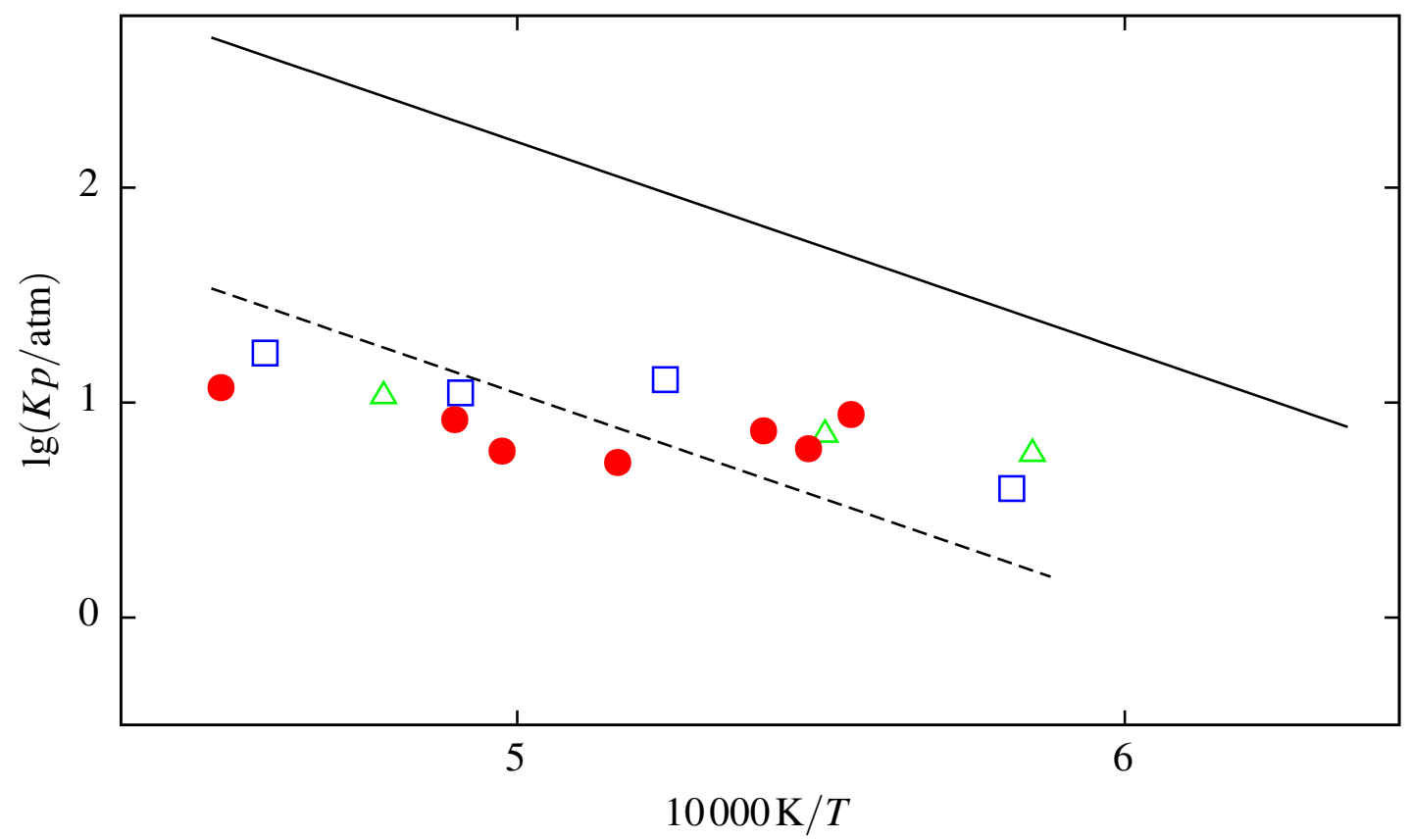

Abbildung 4.3: Berechnete Gleichgewichtskonstanten (durchgezogene Gerade) für die Reaktion $\mathrm{C}_{2} \mathrm{H}_{4} \rightleftharpoons \mathrm{H}_{2}+\mathrm{C}_{2} \mathrm{H}_{2}$ und experimentelle Werte aus $\bullet$ : Benzol-, $\triangle$ : Ethylen- und $\square$ : Hexanpyrolysen bei $P=(5,0 \pm 0,5) \mathrm{MPa}$ und $[C]=(5 \pm 1) \mathrm{mol} / \mathrm{m}^{3}$.

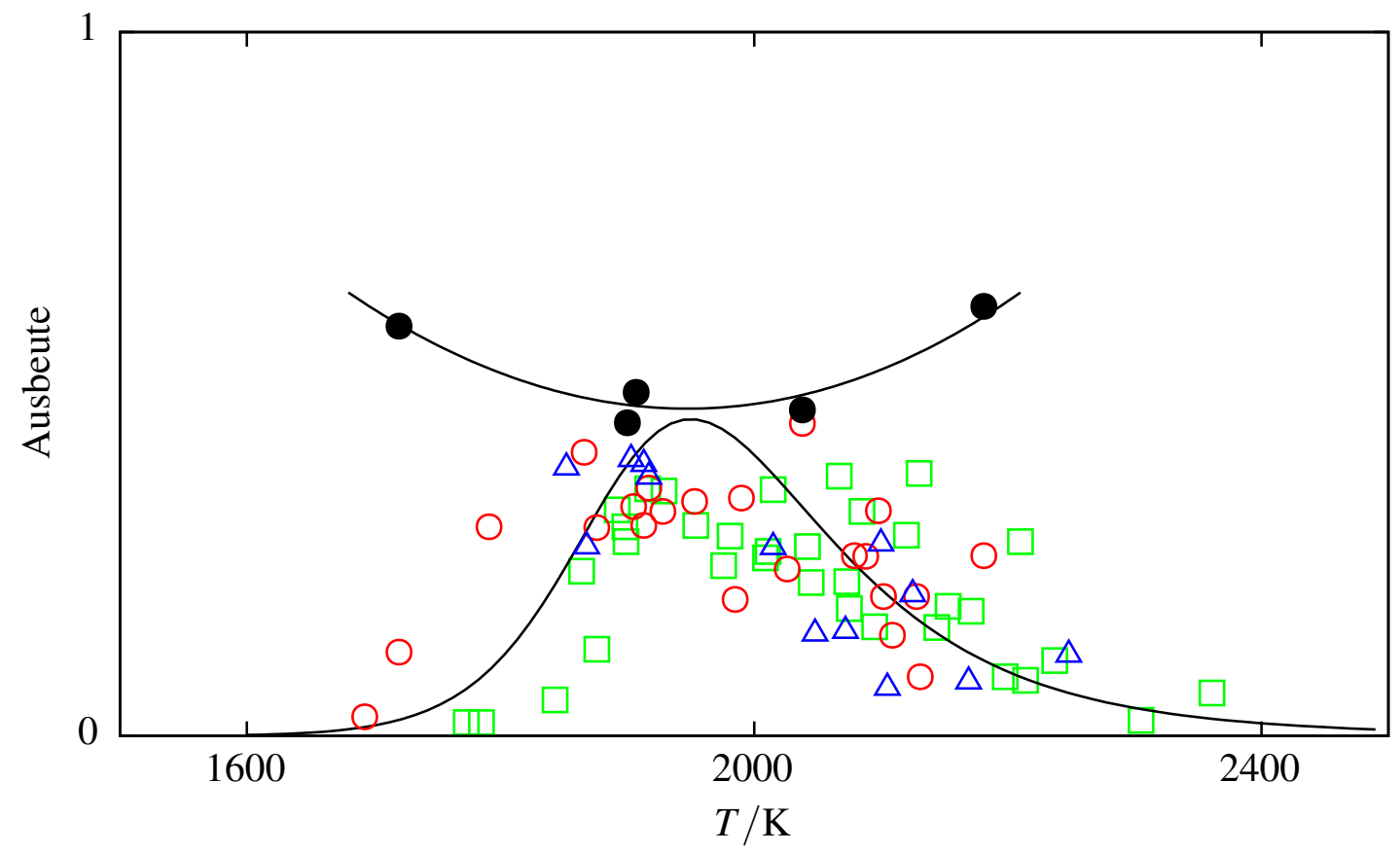

Abbildung 4.4: Temperaturabhängigkeit der Ruß- ( $)$ und Acetylenausbeute (•) in der Hexanpyrolyse. Die mittlere Kohlenstoffkonzentration beträgt $5,3 \mathrm{~mol} / \mathrm{m}^{3}$. 
werden, siehe Abbildung (3.46). In Experimenten bei $1900 \mathrm{~K}, 5 \mathrm{MPa}$ und einer Kohlenstoffkonzentration von $40 \mathrm{~mol} / \mathrm{m}^{3}$ betrug die Rußausbeute in Hexanpyrolysen bis zu $50 \%$ und in Benzolpyrolysen bis $90 \%$. Außerdem nahm in der Hexanpyrolyse die Temperatur maximaler Rußausbeute von $1950 \mathrm{~K}$ auf ca. $T_{\max }^{R A}=2300 \mathrm{~K}$ zu. Dabei erhöhte sich die maximale Rußausbeute von 40 auf $75 \%$.

Über Produktanalysen in Acetylenpyrolysen wurde von Colket et al [40] berichtet. Die Experimente wurden im Single-Puls-Stoßrohr hinter einfallenden Stoßwellen bei einem Druck von 8 atm und mit einem Acetylenmolenbruch von 3,7\% ausgeführt. Die Gasproben wurden innerhalb von $300 \mathrm{~ms}$ über ein Magnetventilsystem aus dem Stoßrohr entnommen und anschließend gaschromatographisch auf Wasserstoff und $\mathrm{C}_{1}$ bis $\mathrm{C}_{10}$ Kohlenwasserstoffe untersucht. Neben Wasserstoff wurden als Hauptprodukte Acetylen $\left(\mathrm{C}_{2 n} \mathrm{H}_{2}\right)$ mit $1 \leq n \leq 4$, und als Nebenprodukte Vinylacetylen, Benzol und Phenylacetylen gefunden. Massenbilanzen wurden nicht angegeben. Im Unterschied zu Acetylenpyrolysen bei höherer Kohlenstoffdichte [41] und in vorgemischten Acetylenflammen [42, 43] wurde von Colket weder Methan, Ethylen noch Ethan gefunden.

Die Methode zur Abschätzung von Rußausbeuten aus Massenbilanzen wurde unter anderem von Kern et al [44, 45, 46, 47] angewendet. Die Kohlenwasserstoffe wurden hinter reflektierten Stoßwellen bei ca. 0,5 atm pyrolysiert und die Produkte im Massenbereich von 12 bis 240 amu zeitaufgelöst $(\Delta t=30 \mu \mathrm{s})$ massenspektrometrisch analysiert. In Acetylenpyrolysen [47] waren Polyacetylene die Hauptprodukte. Ihre Summe war in der Massenbilanz $100 \%$. Somit ergaben sich in Acetylenpyrolysen mit Kohlenstoffdichten kleiner als $1 \mathrm{~mol} / \mathrm{m}^{3}$ vernachlässigbare kleine Rußausbeuten. Ebenso wurden in Toluolpyrolysen $\left([C]=0,3 \mathrm{~mol} / \mathrm{m}^{3}\right)$ Rußausbeuten von unter $10 \%$ festgestellt [46]. Das steht im Gegensatz zu den Ergebnissen dieser Arbeit und den von Thienel gemessenen Rußausbeuten in der Toluolpyrolyse [34]. Für die Acetylenpyrolyse mit der mittleren Kohlenstoffdichte von $0,9 \mathrm{~mol} / \mathrm{m}^{3}$ wurden in Abbildung (3.28) Rußausbeuten von über $50 \%$ angegeben. In Übereinstimmung mit dieser Arbeit, wurden in Benzolpyrolysen mit geringer Kohlenstoffdichte [45], Rußausbeuten von bis zu ca. $50 \%$ berechnet und bei $2000 \mathrm{~K}$ betrug die Acetylenausbeute ca. $40 \%$.

Die Acetylenkonzentration im Abgas von fetten vorgemischten Ethylenflammen hängt nur wenig vom Druck ab. Sowohl in Unterdruck- [48] als auch in Hochdruckflammen [26] beträgt die Acetylenkonzentration ca. $0,01 \mathrm{~mol} / \mathrm{m}^{3}$. Dieser Mangel an Acetylen in Hochdruckflammen bedeutet ein Mangel an Rußmassenwachstumspezies. Der experimentelle Befund wurde dazu verwendet, das Ende des Rußmassenwachstums zu erklären [30]. Dagegen wurde in dieser Arbeit bei Ethylenpyrolysen gezeigt, daß bei einer typischen Flammentemperatur von $1900 \mathrm{~K}$, mehr als die einhundertfache Acetylenkonzentration $\left(2 \mathrm{~mol} / \mathrm{m}^{3}\right)$ vorlag. Damit bestand kein Mangel an Rußmassenwachstumspezies. Dieser Unterschied wird mit der fehlenden Oxidation von Acetylen in Pyrolysen erklärt.

Für das Aufhören des Rußmassenwachstums in Gegenwart hoher Acetylenmengen gibt es mindestens zwei Deutungen. Einerseits kann die Oberfläche der Rußpartikel antikatalytsich wirken, weil dort Radikale, die für das Fortschreiten des Rußmassenwachstums von Bedeutung sind, rekombinieren können. Andererseits kann ein partiell eingestelltes Gleichgewicht von Ruß mit Wasserstoff zu Acetylen angenommen werden. Für eine quantitative Beschreibung dieses Ansatzes fehlen die thermodynamischen Daten des Rußes. Deswegen werden hier zwei Grenzfälle betrachtet, mit denen die Bildungs-Gibbsenthalpie von Ruß abgeschätzt werden kann. Zunächst soll Ruß als großes PAH-Molekül betrachtet werden. Für diesen Fall ist die Bildungs-Gibbsenthalpie so hoch, daß die Rußausbeuten in der Größenordnung der Ausbeute von polycyclischen Aromaten $(\ll 1 \%)$ 
liegen. Alternativ wird angenommen, daß sich aufgrund der Strukturähnlichkeit von Ruß und Graphit ihre Bildungs-Gibbsenthalpien ähneln. In diesem Fall wäre die Rußausbeute im untersuchten Temperaturbereich sehr hoch. Experimentell wurden nur in einem relativ schmalen Temperaturbereich hohe Rußausbeuten gefunden. Außerhalb dieses Temperaturbereiches ist die Rußbildung kinetisch kontrolliert. Vermutlich werden die zur Rußbildung notwendigen aromatischen Spezies bei zu niedriger Temperatur zu langsam gebildet und bei hohen Temperaturen zerfallen die Spezies unter Ringöffnung zu schnell [1].

Mit einem Reaktionsmechanismus, der alle wichtigen Reaktionen zur Rußbildung und zum Rußabbau enthält, läßt sich dieser Ansatz bestätigen. Mit dem von Frenklach et al [49] aufgestellten 100-Spezies-500-Reaktionen-Mechanismus, der unter anderem den Aufbau der ersten aromatischen Ringe und deren planares und sphärisches Wachstum über den sogenannten $\mathrm{H}$-Abstraktions/ $\mathrm{C}_{2} \mathrm{H}_{2}$-Additions-Mechanismus beschreibt, wurde die Rußbildung sowohl in der Pyrolyse als auch in vorgemischten Flammen in guter Übereinstimmung mit den experimentellen Meßergebnissen simuliert. Eine gute Simulation der Experimente wurde erreicht, obwohl anzunehmen ist, daß jede der 500 Geschwindigkeitskonstanten mit einem Fehler von $\pm 50 \%$ oder mehr behaftet ist.

\subsection{Induktionszeit}

Die Induktionszeit der Rußbildung wurde für sechs verschiedene Kohlenwasserstoffe im Temperaturbereich zwischen 1600 und $2400 \mathrm{~K}$ untersucht. Ihre Temperaturabhängigkeit wurde aus Arrheniusdiagrammen ermittelt. Für alle in dieser Arbeit untersuchten Kohlenwasserstoffe wurde eine mittlere scheinbare Aktivierungsenergie von $220 \mathrm{~kJ} / \mathrm{mol}$ gefunden. Die Arrhenius-Vorfaktoren der Alkane sind eine Größenordnung höher als die der Aromaten. Die Kohlenstoffkonzentration wurde bis zu zwei Größenordnungen von 0,4 bis $40 \mathrm{~mol} / \mathrm{m}^{3}$, und der Druck von 0,5 bis $24 \mathrm{MPa}$ variiert. In keinem Fall war ein Druckeinfluß auf die Induktionszeit festzustellen. Das bedeutet, daß sich die geschwindigkeitsbestimmende(n) gaskinetischen Reaktion(en), die zur Produktion der ersten Rußpartikel führen, bereits bei $0,5 \mathrm{MPa}$ im Hochdruckbereich befinden. Der Einfluß der Kohlenstoffdichte auf die Induktionszeit $\left(\tau \cdot[C]^{n}\right)$ war vom Typ des pyrolysierten Kohlenwasserstoffs abhängig. Aromaten und Acetylen zeigten die stärkste $(n=0,75)$, Alkane die geringste $(n \approx 0)$ Abhängigkeit von der Kohlenstoffkonzentration.

Die Induktionszeiten in der Ethylenpyrolyse wurden bereits von mehreren Arbeitsgruppen untersucht $[4,50,51]$. In Übereinstimmung mit ihren Ergebnissen im Kohlenstoffkonzentrationsbereich zwischen 2 und $10 \mathrm{~mol} / \mathrm{m}^{3}$ und $1750 \mathrm{~K}$ wurde auch in dieser Arbeit eine Induktionszeit von 1,0 ms gemessen. Jedoch unterscheiden sich die Ergebnisse der Autoren in der Temperatur- und Kohlenstoffkonzentrationsabhängigkeit. Bei der kleinen Kohlenstoffkonzentration von $0,8 \mathrm{~mol} / \mathrm{m}^{3}$ haben Frenklach et al [52, Seite 12] eine Temperatur von $2300 \mathrm{~K}$ für die Induktionszeit von 1,0 ms angegeben. Die scheinbare Aktivierungsenergie betrug $140 \mathrm{~kJ} / \mathrm{mol}$. Von Fussey [50] wurden die Induktionszeiten der Rußbildung in der Pyrolyse von $\mathrm{C}_{2}$ Kohlenwasserstoffen hinter einfallenden Stoßwellen gemessen $(P=0,1$ bis $1,2 \mathrm{MPa})$ und die von ihm gefundene geringe Abhängigkeit der Induktionszeit in der Ethylenpyrolyse von der Kohlenstoffdichte $(n=0,23)$ konnte in dieser Arbeit bestätigt $(n<0,4)$ werden. Dabei betrug die scheinbare Aktivierungsenergie $116 \mathrm{~kJ} / \mathrm{mol}$. Geck [4] bestimmte die Aktivierungsenergie hinter reflektierten Stoßwellen $(P=1$ bis $20 \mathrm{MPa})$ im Mittel zu $245 \mathrm{~kJ} / \mathrm{mol}$ und mit $n=1$ stellte er eine starke Abhängigkeit von der Kohlenstoffdichte fest. D'Alessio et al [51] fanden hinter reflektierten Stoßwellen (0,9bis 1,5 MPa) eine scheinbare Aktivierungsenergie von $142 \mathrm{~kJ} / \mathrm{mol}$. 


\begin{tabular}{cccl}
\hline$E_{\text {Ind }} / \frac{\mathrm{kJ}}{\mathrm{mol}}$ & $n$ & {$[C] / \frac{\mathrm{mol}}{\mathrm{m}^{3}}$} & Literaturquelle \\
\hline 40 bis 70 & 1 bis 2 & 1 bis 5 & Hooker [54] \\
180 & 0,3 & 1 bis 20 & Geck [4] \\
135 & 0,41 & 0,2 bis 2 & Fussey [50] \\
170 & 1 & 1 bis 2 & Kamikaze [53] \\
140 & - & 0,8 & Frenklach [52] \\
216 & 0,75 & 0,9 bis 4 & diese Arbeit \\
\hline
\end{tabular}

Tabelle 4.1: Literaturvergleich der Induktionszeiten in der Acetylenpyrolyse.

Die Induktionszeiten der Rußbildung in der Acetylenpyrolyse wurden schon mehrfach untersucht, siehe Tabelle (4.1). Für die absoluten Zeiten und die Änderung der Induktionszeit mit der Temperatur und der Kohlenstoffkonzentration wurden sehr unterschiedliche Werte angegeben. Yoshizawa et al [53] haben zehnfach längere Induktionszeiten gemessen als Hooker [54].

Innerhalb der Induktionszeit werden Rußvorläufer und erste Rußpartikel gebildet. Wegen der besonders kurzen Induktionszeiten in der Pyrolyse von Aromaten wird angenommen, daß Rußvorläufer aromatische Struktur haben müssen. Die Induktionszeit in der Acetylenpyrolyse ist ca. fünfmal länger als in der Pyrolyse von Aromaten. Damit spielen die Polyacetylene $\left(\mathrm{C}_{2 n} \mathrm{H}_{2}\right)$, die in großer Menge in Acetylenpyrolysen gebildet werden [40], nur eine untergeordnete Rolle als Rußvorläufer.

\subsection{Vergleich mit vorgemischten Flammen}

Die charakteristischen Größen des Rußmassenwachstums $\left(k_{f}\right.$ und $\left.f_{v \infty}\right)$ sind in vorgemischten Ethylen/Luft Flammen mit großer Variation von Temperatur, Druck und Mischungszusammensetzung untersucht worden.

\subsubsection{Rußmassenwachstumskonstante}

Zum Vergleich der formalen Rußbildungsgeschwindigkeitskonstante $k_{f}$ mit den Stoßwellendaten wird $k_{f}$ auf die sogenannte Überschuß-Kohlenstoffdichte normiert. Das ist die Kohlenstoffdichte, die in der Flamme für die Rußbildung zur Verfügung steht. Zur Bestimmung der Überschuß-Kohlenstoffdichte wurden zwei verschiedene Methoden vorgeschlagen $[31,55]$.

- Die Überschuß-Kohlenstoffdichte wird aus der Differenz von $\left[(\mathrm{C} / \mathrm{O})-(\mathrm{C} / \mathrm{O})_{\mathrm{krit}}\right]$ berechnet. Das $(\mathrm{C} / \mathrm{O})$-Verhältnis ergibt sich aus dem Verhältnis der Kohlenstoffund Sauerstoffkonzentration der Flamme. $(\mathrm{C} / \mathrm{O})_{\text {krit }}$ ist das $(\mathrm{C} / \mathrm{O})$-Verhältnis an der Rußgrenze. Die nach dieser Methode berechnete Überschuß-Kohlenstoffdichte wurde in der Dissertation von Feldermann [31] mit $[\mathrm{C}]_{\text {surplus }}$ bezeichnet. $[\mathrm{C}]_{\text {surplus }}$ wird für Kohlenwasserstoff/Luft Flammen mit Gleichung (4.17) berechnet.

$$
[\mathrm{C}]_{\text {surplus }}=\frac{P}{R T} \cdot \frac{1}{M V} \cdot\left(1-\frac{(\mathrm{C} / \mathrm{O})_{\mathrm{krit}}}{(\mathrm{C} / \mathrm{O})}\right) \cdot\left(\frac{1}{n}+\frac{1}{2 \cdot x_{\mathrm{O}_{2}} \cdot(\mathrm{C} / \mathrm{O})}\right)^{-1}
$$

$n$ ist die Anzahl der C-Atome im Kohlenwasserstoffmolekül, $x_{\mathrm{O}_{2}}=0,2095$ ist der Sauerstoff-Molenbruch der Luft und $M V \approx(1,1 \pm 0,1)$ ist die Molzahlvermehrung während der Verbrennung. 


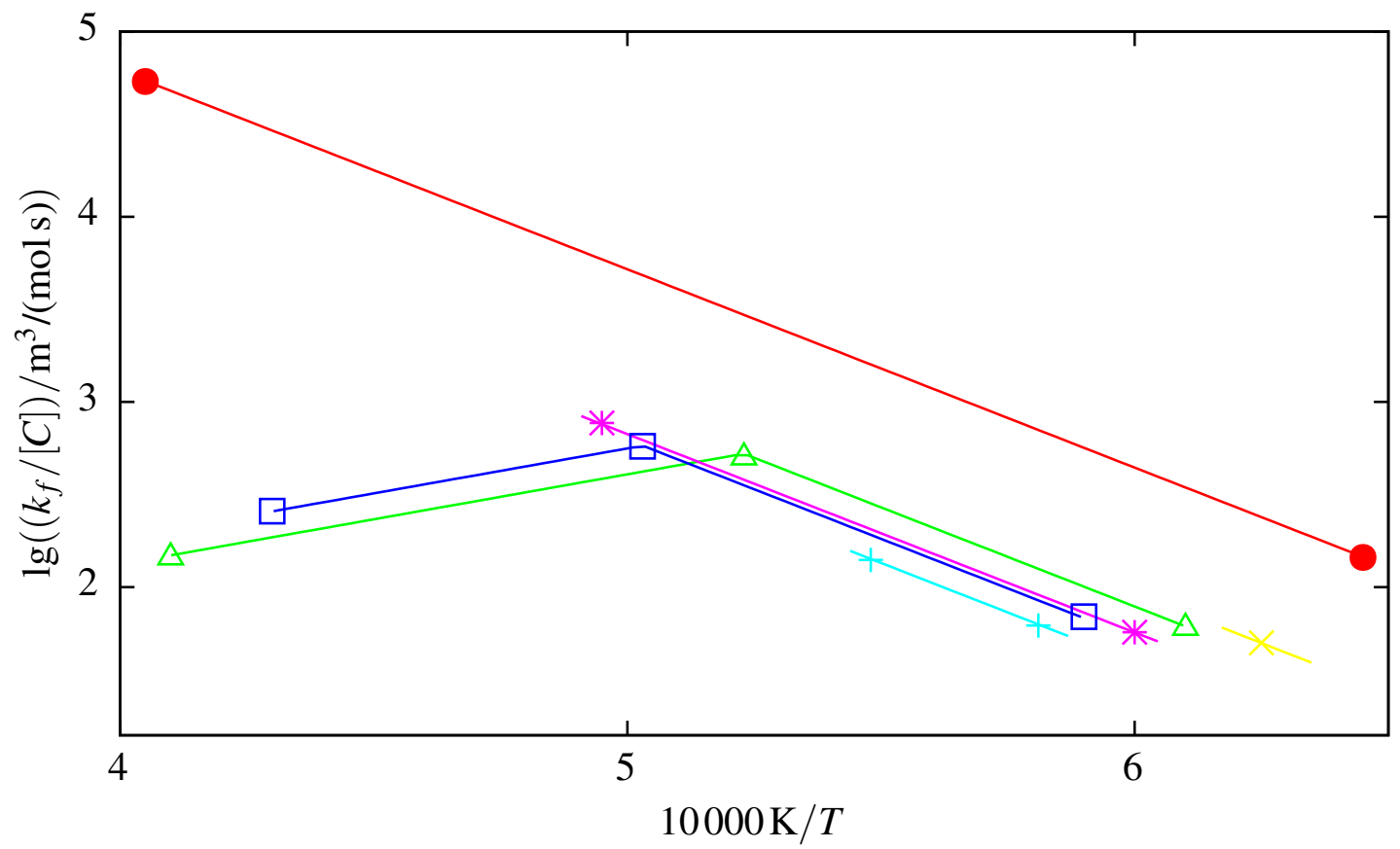

Abbildung 4.5: Vergleich der normierten Massenwachstumskonstante $\left(k_{f} /[C]\right)$ in Abhängigkeit der Temperatur für Benzol- Ethylen- und Hexanpyrolysen sowie Benzolund Ethylenflammen. Die angegebenen Konzentrationen beziehen sich auf die C-Atome. $\times$ : Normaldruck-Benzol-Luft-Flamme 0,3 bis $0,7 \mathrm{~mol} / \mathrm{m}^{3}+$ : Normaldruck-Ethylen-Sauerstoff-Flamme 0,5 bis $0,8 \mathrm{~mol} / \mathrm{m}^{3}$ und $*$ : $1 \mathrm{MPa}$-Ethylen-Luft-Flamme $0,4 \mathrm{bis} 3 \mathrm{~mol} / \mathrm{m}^{3}$. •: Benzol 0,4 bis $4,0 \mathrm{~mol} / \mathrm{m}^{3} \triangle$ : Ethylen 3,9 bis $12 \mathrm{~mol} / \mathrm{m}^{3}$ und $\square$ : $\mathrm{n}$-Hexan 5,3 bis $35 \mathrm{~mol} / \mathrm{m}^{3}$.

- Wagner et al [55] berechneten aus Gaschromatograph-Analysen, die parallel zu den Absorptionmessungen aus Flammenabgasen ausgeführt wurden, eine „effektive“ Kohlenstoffdichte. Von der Gesamt-Kohlenstoffdichte wird die Kohlenstoffdichte abgezogen, die sich in für die Rußbildung ,,inerten“ Molekülen befindet. Als inert werden Kohlendioxid, Kohlenmonoxid und Methan betrachtet.

$$
[\mathrm{C}]_{\text {effektiv }}=[\mathrm{C}]_{\text {ges }}-\left[\mathrm{CO}_{2}\right]-[\mathrm{CO}]-\left[\mathrm{CH}_{4}\right]
$$

Kohlendioxid und Kohlenmonoxid sind thermodynamisch stabile Endprodukte, und Methan ist der einzige Kohlenwasserstoff, der in vorgemischten NormaldruckLuftflammen besonders wenig zur Rußbildung neigt.

Bei Drücken bis zu $1 \mathrm{MPa}$ ist in Ethylenflammen die „Surplus“-Kohlenstoffdichte über einen weiten Bereich des (C/O)-Verhältnisses ca. doppelt so groß wie die effektive Kohlenstoffdichte: $[\mathrm{C}]_{\text {surplus }} \approx 2 \cdot[\mathrm{C}]_{\text {effektiv }}$. In dieser Arbeit wird für Flammen $[\mathrm{C}]_{\text {effektiv }}$ als Maß für die zur Rußbildung zur Verfügung stehende Kohlenstoffdichte verwendet. Für die Fälle, in denen zu den Absorptionmessungen keine GC-Analysen vorlagen, wurde stattdessen $[\mathrm{C}]_{\text {surplus }}$ verwendet. Abbildung (4.5) zeigt die normierten Rußbildungsgeschwindigkeitskonstanten $\left(k_{f} /[C]\right)$ für die Rußbildung in Benzol- Ethylen- und Hexanpyrolysen sowie in einer Benzolflamme [43] und zwei Ethylen-Flammen [31, 56]. Die Rußbildungsgeschwindigkeitskonstante der Benzolflamme wurde auf $[\mathrm{C}]_{\text {surplus }}$ normiert. Die normierten Rußbildungsgeschwindigkeitskonstanten in den Ethylenflammen 
unterscheiden sich im gesamten Temperaturmeßbereich (1700 bis $2000 \mathrm{~K})$ um weniger als $\pm 50 \%$ von den Rußbildungsgeschwindigkeitskonstanten in der Pyrolyse der Alkane und Ethylen. Offenbar haben die sauerstoffhaltigen Spezies in Ethylenflammen nur einen vernachlässigbaren kleinen Einfluß auf $\left(k_{f} /[C]\right)$. Das Rußmassenwachstum verläuft in Benzolflammen ähnlich langsam wie in in der Pyrolyse der Alkane und in Ethylenflammen. Die im Vergleich zur Benzolpyrolyse fünffach kleinere normierte Rußbildungsgeschwindigkeitskonstante in Benzolflammen wird mit der chemischen Umgebung erklärt, in der die Rußpartikel wachsen. Das Rußmassenwachstum setzt erst hinter der Reaktionszone in ca. $10 \mathrm{~mm}$ Höhe über der Brenneroberfläche ein. Hier enthält die chemische Umgebung qualitativ dieselben Hauptprodukte (Wassergase, Acetylen, Methan, Ethylen) wie die Ethylenflamme.

In den Arrhenius-Auftragungen (4.5 und 3.24) sind die Maxima der normierten Massenwachstumskonstante $\left(k_{f} /[C]\right)$ bei der Temperatur von $(1950 \pm 50) \mathrm{K}$ besonders auffällig. Diese Maxima zeigten alle untersuchten Kohlenwasserstoffe sowie Styrol und Toluol [34]. Nur für die Benzolpyrolysen und die Flammenexperimente wurde kein Maximum gefunden. In den vorgemischten Flammen kann dieses Maximum nicht beobachtet werden, weil Flammentemperaturen von über $2000 \mathrm{~K}$ kaum erreicht werden konnten. In den Benzolpyrolysen wurde bis $2400 \mathrm{~K}$ kein Maximum gefunden. In Pyrolysen, die bei Temperaturen über $2400 \mathrm{~K}$ ausgeführt wurden, war die gebildete Rußmenge zu klein und die Zeitauflösung zu gering um aus den Rußzeitprofilen die formale Rußbildungsgeschwindigkeitskonstante zu finden. Ein größerer Anteil des Benzols wird offenbar innerhalb der Induktionszeit nicht pyrolysiert und nimmt anschließend als Wachstumsspezies am schnellen Rußmassenwachstum teil.

\subsubsection{Rußausbeute}

Der Rußausbeute nimmt in vorgemischten Ethylenflammen mit zunehmender ÜberschußKohlenstoffdichte stark zu [31, 57, 58]. Mit einem zu Gleichung (3.11) äquivalenten Ansatz wurde die Abhängigkeit vom $(\mathrm{C} / \mathrm{O})$-Verhältnis bzw. von $[\mathrm{C}]_{\text {surplus }}^{n}$ beschrieben.

$$
R A_{\infty} \propto[\mathrm{C}]_{\text {surplus }}^{n} \quad \text { mit: } \quad n \approx 2
$$

Die Rußausbeute nimmt quadratisch mit der Überschuß-Kohlenstoffdichte zu. In dieser Arbeit wurde im untersuchten Bereich der Kohlenstoffdichte für Ethylen- und Methanpyrolysen derselbe Exponent gefunden. Dieser starke Einfluß der Kohlenstoffkonzentration auf die Rußausbeute $m u \beta$ im Bereich hoher Rußausbeuten kleiner werden $n \rightarrow 0$. Andernfalls würde die theoretische Rußausbeute auf über $100 \%$ zunehmen. Besonders hohe Rußausbeuten wurden in den Pyrolysen von Acetylen und Benzol gemessen. Hier nahm die Rußausbeute nur noch proportional mit der Kohlenstoffkonzentration zu.

Auch die Temperaturabhängigkeit der Rußausbeute in Flammenexperimenten ist der in Pyrolyseexperimenten ähnlich. In der Diplomarbeit von Weiß [59] wurde eine „Glockenkurve“ für Ethylen-Luft-Flammen dargestellt. Die maximale Rußausbeute beträgt bei $T_{\max }^{R A}=1600 \mathrm{~K}$ ca. $7 \%$. In vielen Ethylen-Flammen wurde häufig nur die Hochtemperaturseite der Glockenkurven bestimmt [31, 56, 60]. Die Maximumtemperatur ist $T_{\max }^{R A} \approx 1600 \mathrm{~K}$ und die Rußausbeuten erreichen Werte von $0,1 \%$ bis zu $40 \%$. Im Vergleich dazu wurden bei Ethylen-Pyrolysen Rußausbeuten bis zu $90 \%$ gemessen und die Maximumtemperatur ist um $250 \mathrm{~K}$ höher. Messungen bei niedrigen Flammmentemperaturen sind wegen der niedrigen Flammenstabilität schwer zugänglich. 


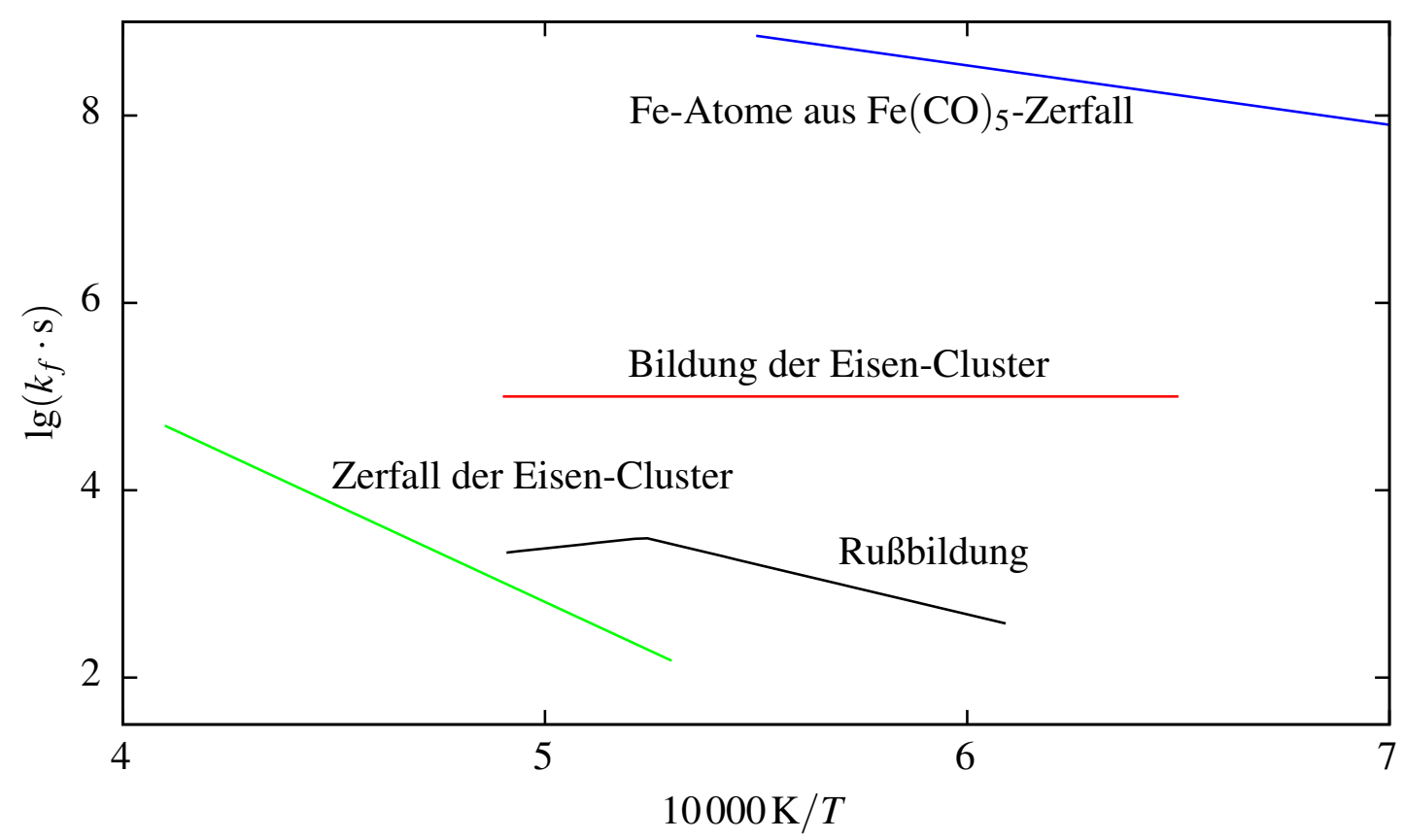

Abbildung 4.6: Charakteristische Geschwindigkeitskonstanten erster Ordnung für die Eisenatombildung in der Pyrolyse von Eisenpentacarbonyl, die Bildung und den Zerfall von Eisenclustern sowie das Rußmassenwachstum in der Ethylenpyrolyse in dem für die Rußbildung relevanten Temperaturbereich.

\subsection{Einfluß von Eisenpentacarbonyl}

Die Experimente zeigten, daß die Rußbildung in Ethylenpyrolysen durch die Anwesenheit von 200 ppm Eisenpentacarbonyl wenig beeinflußt wurde. Die Konzentration von Eisenclustern lag in den Experimenten hinter reflektierten Stoßwellen im Bereich von $10^{19} / \mathrm{m}^{3}$. Obwohl das die Größenordnung von der Rußpartikelanzahldichte $N_{0}$ in der Frühphase der Rußbildung ist, dienten die Eisencluster als wenig wirksame Kondensationskeime für Rußmassenwachstumspezies. Es wurden nur kleine Rußausbeuten $(R A<5 \%)$ unmittelbar nach Eintreffen der reflektierten Stoßwelle erzeugt. Die „normalen“ Induktionszeiten wurden nicht verkürzt und die nach der Induktionsperiode erzeugte Rußausbeute war um ca. 1/3 höher als in Experimenten ohne Eisenpentacarbonyl. In Experimenten hinter einfallenden Stoßwellen lagen unmittelbar am Beginn der Pyrolyse keine Eisencluster und somit keine Kondensationskeime vor. Der Zerfall von Eisenpentacarbonyl und die sich anschließende Bildung der Eisencluster finden auf einer kürzeren Zeitskala statt als die Rußbildung [22, 61], siehe Abbildung (4.6). Somit konnte die Rußbildung ähnlich gefördert werden wie hinter reflektierten Stoßwellen. In vorgemischten Ethylenflammen wurde der Eiseneinfluß der Rußbildung von Ritrievi [62] und Feitelberg [63] untersucht. Ein kleiner Anteil Ferrocen (200 ppm), das zum Frischgas zugemischt wurde, erhöhte die Rußausbeute bis zu einem Faktor von drei. Im Gegensatz dazu fanden Bonczyk [64] und Mitchell [65] in Diffusionsflammen eine Abnahme der Rußausbeute. 


\section{Literaturverzeichnis}

[1] S. C. Graham, J. B. Homer und J. L. J. Rosenfeld, Proc. R. Soc. Lond. A., 344:259, 1975. 1, 8, 17, 18, 35, 59, 66, 76

[2] M. Frenklach, S. Taki und R. A. Matula, Combustion and Flame, 49:275, 1983. 1, $17,18,35$

[3] B. Simmons und A. Williams, Combustion and Flame, 71:219, 1988. 1

[4] C. C. Geck, Diplomarbeit, Universität Göttingen, 1975. 1, 16, 17, 18, 21, 28, 66, 67

[5] S. M. Hwang, P. Vlasov, H. Gg. Wagner und Th. Wolff, Z. Phys. Chem. Neue Folge, 137:129-139, 1991. 1, 35

[6] H. Oertel, Stoßrohre, Springer-Verlag, 1966. 3, 4, 5, 77

[7] A. G. Gaydon und I. R. Hurle, The Shock Tube in High-Temperature Chemical Physics, Chapman and Hall L.T.D., London, 1963. 3

[8] E. F. Greene und J. P. Toennies, Chemische Reaktionen in Stoßwellen, Dr. Dietrich Steinkopff Verlag, Darmstadt, 1959, translated by H. Gg. Wagner. 3

[9] H. Henrici, Dissertation, Universität Göttingen, 1966. 6

[10] H. A. Olschewski, J. Troe und H. Gg. Wagner, Ber. Bunsenges. Physk. Chem., 70:1060, 1966. 6

[11] Th. Wolff, Dissertation, Universität Göttingen, 1990. 6

[12] H. C. van de Hulst, Light Scattering by Small Particles, Wiley, New York, 1957. 8

[13] M. Kerker, The Scattering of Light, Academic Press, New York, 1969. 8, 57, 58

[14] W. H. Dalzell und A. F. Sarofim, Trans. ASME., J. Heat Transfer, 91:100-104, 1969. 8

[15] S. C. Lee und C. L. Tien, Optical Constants of Soot in Hydrocarbon Flames, in Eighteenth Symposium (International) on Combustion, S. 1159-1166, The Combustion Institute, Pittsburgh, 1980. 9, 76

[16] S. Hanisch, Dissertation, Universität Göttingen, 1994. 13, 59

[17] K. Spindler, Dissertation, Universität Göttingen, 1986. 13

[18] B. S. Haynes und H. Gg. Wagner, Prog. Energy Combust. Sci., 7:229, 1981. 16, 58 
[19] Dietmar Tanke, Zeitaufgelöste Absorptionsmessungen bei der Rußbildung aus nHexan und Benzol hinter Hochdruck-Stoßwellen, Diplomarbeit, Universität Göttingen, Okt. 1991. 16, 19, 24, 30, 40

[20] W. Buckendahl, Diplomarbeit, Universität Göttingen, 1970. 17, 18

[21] A. Müller, Dissertation, Universität Karlsruhe, 1991. 18

[22] I. S. Zaslonko, private Mitteilung, Göttingen, 1994. 43, 70

[23] Prof. Tesner, private Mitteilung, Moskau, 1994. 54, 77

[24] Dr. Wilbrandt, private Mitteilung, Göttingen, 1993. 56

[25] A. Heddrich, Dissertation, TH Darmstadt, 1986. 57

[26] B. Lüers-Jongen, Dissertation, Universität Göttingen, 1990. 57, 65

[27] S. Hanisch, H. Jander, Th. Pape und H. Gg. Wagner, in Twenty-Fifth Symposium (International) on Combustion, The Combustion Institute, Pittsburgh, 1994. 58, 59

[28] Th. Pape, Diplomarbeit, Universität Göttingen, 1993. 58

[29] Th. Baum, S. Löffler, Ph. Löffler, P. Weilmünster und K. H. Homann, Ber. Bunsenges. Physk. Chem., 96:841, 1992. 58

[30] H. Böhm, Ch. Feldermann, Th. Heidermann, H. Jander, B. Lüers und H. Gg. Wagner, in Twenty-Fourth Symposium (International) on Combustion, S. 991, The Combustion Institute, Pittsburgh, 1992. 58, 65

[31] Ch. Feldermann, Dissertation, Universität Göttingen, 1992. 58, 67, 68, 69

[32] G. M. Hidy und J. R. Brock, The Dynamics of Aerocolloidal Systems, Pergamon Press, New York, 1979. 58, 59

[33] J. Schulz, Dissertation, Universität Göttingen, 1987. 59

[34] Th. Thienel, private Mitteilung, Göttingen, 1994. 60, 65, 69

[35] A. A. Frost und R. G. Pearson, Kinetik und Mechanismen homogener chemischer Gasreaktionen, Verlag Chemie, Weinheim, 1964. 60

[36] Robert J. Kee, Fran M. Ruply und James A. Miller, The Chemkin Thermodynamic Data Base, Sandia Report SAND87-8215B, Sandia National Laboratories, Livermore, California, März 1990, http://www.ca.sandia.gov/chemkin/. 62

[37] D. R. Stull und H. Prophet, JANAF Thermochemical Tables, Nat. Stand. Ref. Data Ser., Nat. Bur. Stand, 1971. 62

[38] S. G. et al Lias, NIST Standard Reference Database 25 Version 2.01, Techn. Ber., NIST, Gaithersburg, 1994. 62

[39] I. Barin, Thermochemical Data of Pure Substances, VCH, Weinheim, 2. Aufl., 1993. 62 
[40] B. Meredith und M. B. Colket, in Twenty-First Symposium (International) on Combustion, S. 851, The Combustion Institute, Pittsburgh, 1986. 65, 67

[41] F. Stephan, Diplomarbeit, Universität Göttingen, 1994. 65

[42] M. Brei, Dissertation, Universität Göttingen, 1985. 65

[43] D. Hesse, Dissertation, Universität Göttingen, 1988. 65, 68

[44] R. D. Kern, H. J. Singh, M. A. Esslinger und P. W. Winkeler, in Nineteenth Symposium (International) on Combustion, S. 1351, The Combustion Institute, Pittsburgh, 1982. 65

[45] H. J. Singh und R. D. Kern, Combustion and Flame, 54:49, 1983. 65

[46] R. D. Kern, C. H. Wu, G. B. Skinner, V. S. Rao, J. H. Kiefer, J. A. Towers und L. J. Mizerka, in Twenthieth Symposium (International) on Combustion, S. 789, The Combustion Institute, Pittsburgh, 1984. 65

[47] C. H. Wu, H. J. Singh und R. D. Kern, Journal of Chemical Kinetics, 19:975, 1987. 65

[48] U. Bonne und H. Gg. Wagner, Ber. Bunsenges. Physk. Chem., 69:503, 1965. 65

[49] A. Kazakov, H. Wang und M. Frenklach, in Twenty-Fifth Symposium (International) on Combustion, The Combustion Institute, Pittsburgh, 1994. 66

[50] D. E. Fussey, A. J. Gosling und D. Lampard, Combustion and Flame, 32:181, 1978. 66,67

[51] A. D'Alessio und P. Massoli, in Twenty-Fifth Symposium (International) on Combustion, The Combustion Institute, Pittsburgh, 1994. 66

[52] M. Frenklach, D. W. Clary und M. K. Ramachandra, Contractor Report 174880, NASA, 1985. 66, 67

[53] Y. Yoshizawa, H. Kawada und M. Kurokawa, in Seventeenth Symposium (International) on Combustion, 1978. 67

[54] W. J. Hooker, in Seventh Symposium (International) on Combustion, S. 949, The Combustion Institute, Pittsburgh, 1958. 67

[55] Stefan Bauerle, Yuri K. Karasevitch, Stefan Slavov, Dietmar Tanke, Matthias Tappe, Thomas Thienel und Heinz Gg. Wagner, Soot Formation at Elevated Pressures and Carbon Concentrations in Hydrocarbon Pyrolysis, in Twenty-Fifth Symposium (International) on Combustion, S. 627-634, The Combustion Institute, Pittsburgh, University of California, Irvine, Aug. 1994. 67, 68

[56] N. Petereit, Dissertation, Universität Göttingen, 1992. 68, 69

[57] H. Gg. Wagner, Agard Report 422/22, Nato, 1988. 69

[58] J. Lahaye und G. Prado, Soot in Combustion Systems and its Toxic Properties, Plenum Press, New York and London, 1983. 69 
[59] M. Weiß, Diplomarbeit, Universität Göttingen, 1986. 69

[60] H. Mätzing, Dissertation, Universität Göttingen, 1986. 69

[61] D. Tanke, H. Gg. Wagner und I. S. Zaslonko, wird zur Veröffentlichung eingereicht. 70

[62] K. E. Ritrievi, J. P. Longwell und A. F. Sarofim, Combustion and Flame, 70:17, 1987. 70

[63] A. S. Feitelberg, J. P. Longwell und A. F. Sarofim, Combustion and Flame, 92:241, 1993. 70

[64] P. A. Bonczyk, Combustion and Flame, 87:233, 1991. 70

[65] J. B. A. Mitchell, D. J. M. Miller und M. Sharpe, Combust. Sci. Technol., 74:63, 1990. 70

[66] J. E. Smith und M. L. Jordan, Journal of Colloid Science, 19:549, 1964. 75 


\section{Kapitel 5}

\section{Anhang}

\subsection{Logarithmisch normale Verteilungsfunktion}

Die Durchmesser von polydispersen Systemen (z. B. Aerosolen) sind häufig logarithmisch normal verteilt [66]. Für eine logarithmisch normal verteilte Wahrscheinlichkeit $P$ einer Zufallsgröße $x$ gilt, daß der natürliche Logarithmus der Zufallsgröße $\ln (x)$ GaußNormalverteilt ist.

$$
P(x)=\int_{a}^{b} p(x) \mathrm{d} x=\int_{a}^{b} \frac{1}{\sqrt{2 \pi} \sigma_{g}} \cdot \exp \left[-\frac{\ln ^{2}\left(x / x_{g}\right)}{2 \sigma_{g}^{2}}\right] \mathrm{d} \ln (x)
$$

Die Parameter der Funktion sind das geometrische Mittel $x_{g}$ und die geometrische Standardabweichung $\sigma_{g}$.

$$
\sigma_{g}^{2}=\int_{\rightarrow 0}^{\infty} \ln ^{2}\left(x / x_{g}\right) \cdot p(x) \mathrm{d} x
$$

Mit Hilfe der ersten Ableitung wird der Modalwert $x_{M}$ bestimmt.

$$
\begin{gathered}
\frac{\mathrm{d} p(x)}{\mathrm{d} x}=-\frac{p(x)}{x} \cdot\left(1+\frac{\ln \left(x / x_{g}\right)}{\sigma_{g}^{2}}\right) \\
\ln x_{M}=\ln x_{g}-\sigma_{g}^{2} \\
p\left(x_{M}\right)=\frac{\exp \left(\sigma_{g}^{2} / 2\right)}{\sqrt{2 \pi} \cdot \sigma_{g} \cdot x_{g}}
\end{gathered}
$$

Der Median $x_{m}$ ist gleich dem geometrischem Mittelwert $x_{g}$. Aus den Momenten $\alpha_{n}$ werden die Mittelwerte $x_{n m}$ berechnet.

$$
\begin{aligned}
& \alpha_{n}=\int_{\rightarrow 0}^{\infty} x^{n} \cdot p(x) \mathrm{d} x=x_{g}^{n} \cdot \exp \left(\frac{n^{2} \cdot \sigma_{g}^{2}}{2}\right) \\
& x_{n m}=\left(\frac{\alpha_{n}}{\alpha_{m}}\right)^{\frac{1}{n-m}}=x_{g} \cdot \exp \left(\frac{n+m}{2} \cdot \sigma_{g}^{2}\right)
\end{aligned}
$$


Der mittlere Durchmesser von Rußpartikel läßt sich aus einer Kombination von Streuund Absorptionsexperimenten bestimmen. Der Streuquerschnitt ist proportional zur sechsten Potenz und die Absorption proportional zur dritten Potenz des Durchmessers. Damit gilt folgender Zusammenhang zwischen dem geometrischem Mittel $d_{g}$ und dem optisch bestimmten Durchmesser $d_{36}$.

$$
d_{36}=d_{g} \cdot \exp \left(4.5 \cdot \sigma_{g}^{2}\right)
$$

\subsection{Symbolverzeichnis}

Tabelle 5.1: Die wichtigsten Symbole, in thematischer Sortierung mit Angabe typischer Werte.

\begin{tabular}{|c|c|c|c|}
\hline Symbol & typ. Werte & Einheit & Bedeutung \\
\hline \multicolumn{4}{|l|}{ Testgas } \\
\hline$\dot{x}_{S}$ & 1000 & $\mathrm{~m} / \mathrm{s}$ & $\begin{array}{l}\text { Geschwindigkeit der einfallenden Stoß- } \\
\text { welle, siehe Abbildung (2.1) auf Seite } 5\end{array}$ \\
\hline Indizes $1,2,2 \mathrm{R}$ & - & - & $\begin{array}{l}\text { Laufgas vor sowie hinter der einfallenden } \\
\text { und reflektierten Stoßwelle }\end{array}$ \\
\hline Indizes $3,4,5$ & - & - & $\begin{array}{l}\text { Treibgas hinter der Mediengrenze sowie } \\
\text { vor und hinter den Expansionswellen }\end{array}$ \\
\hline$a_{L}$ und $a_{T}$ & 308,1261 & $\mathrm{~m} / \mathrm{s}$ & $\begin{array}{l}\text { Schallgeschwindigkeit des Laufgases Ar } \\
\text { und Treibgases } \mathrm{H}_{2}\end{array}$ \\
\hline$f_{L}$ und $f_{T}$ & 3 und 5 & - & Freiheitsgrade des Lauf- und Treibgases \\
\hline$\kappa=\left(c_{p} / c_{v}\right)$ & $(1+2 / f)$ & - & Verhältnis der spezifischen Wärmen \\
\hline$M$ & 2 bis 5 & - & MACH-Zahl \\
\hline$t$ & 2 & $\mathrm{~ms}$ & Meßzeit hinter der Stoßwelle \\
\hline$T$ & $2000 \pm 500$ & $\mathrm{~K}$ & Temperatur hinter der Stoßwelle \\
\hline$P$ & 0,25 bis 25 & $\mathrm{MPa}$ & Druck hinter der Stoßwelle \\
\hline$x\left(\mathrm{C}_{\mathrm{n}} \mathrm{H}_{\mathrm{m}}\right)$ & 0,02 bis 2 & $\%$ & $\begin{array}{l}\text { Molenbruch oder Stoffmengenanteil von } \\
\text { Kohlenwasserstoff }\end{array}$ \\
\hline$(\mathrm{C} / \mathrm{O})$ & $0,7 \pm 0,3$ & - & $\mathrm{C}$ zu O-Verhältnis einer fetten Flamme \\
\hline$(\mathrm{C} / \mathrm{O})_{\text {krit }}$ & $0,6 \pm 0,2$ & - & $(\mathrm{C} / \mathrm{O})$ an der Rußgrenze \\
\hline$[\mathrm{C}]$ & 0,5 bis 50 & $\mathrm{~mol} / \mathrm{m}^{3}$ & Kohlenstoffkonzentration \\
\hline$[\mathrm{C}]_{\text {surplus }}$ & 0,1 bis 10 & $\mathrm{~mol} / \mathrm{m}^{3}$ & $\begin{array}{l}\text { Überschuß-Kohlenstoffdichte berechnet } \\
\text { aus }(\mathrm{C} / \mathrm{O}) \text {, siehe Gl. (4.17) auf Seite } 67\end{array}$ \\
\hline$[\mathrm{C}]_{\text {effektiv }}$ & 0,05 bis 5 & $\mathrm{~mol} / \mathrm{m}^{3}$ & $\begin{array}{l}\text { Effektive Kohlenstoffdichte berechnet aus } \\
\text { Gasanalysen, siehe Gl. (4.18) auf Seite } 68\end{array}$ \\
\hline \multicolumn{4}{|l|}{ Rußpartikel } \\
\hline$\rho_{\mathrm{Ru} ß}$ & 1860 & $\mathrm{~kg} / \mathrm{m}^{3}$ & Spezifische Dichte [1] \\
\hline$m(632,8 \mathrm{~nm})$ & $n-i \cdot k$ & - & Brechungsindex \\
\hline$n$ & 1,9 & - & Realteil von $m$ [15] \\
\hline$k$ & 0,5 & - & Imaginärteil von $m$ [15] \\
\hline$\alpha(632,8 \mathrm{~nm})$ & 30 bis 50 & $\mathrm{~m}^{2} / \mathrm{mol}$ & $\begin{array}{l}\text { Natürlicher Extinktionskoeffizient, siehe } \\
\text { Gleichung (2.11) auf Seite } 8\end{array}$ \\
\hline
\end{tabular}


Tabelle 5.1: Die wichtigsten Symbole (Fortsetzung)

\begin{tabular}{|c|c|c|c|}
\hline$d_{g}$ & 30 & $\mathrm{~nm}$ & Geometrischer Partikeldurchmesser \\
\hline$\sigma_{g}$ & 0,2 & - & Geometrische Standardabweichung \\
\hline $\bar{v}$ & $10^{-23}$ & $\mathrm{~m}^{3}$ & Mittleres Partikelvolumen \\
\hline$(A / m)$ & 110 & $\mathrm{~m}^{2} / \mathrm{g}$ & spezifische Oberfläche [23] \\
\hline \multicolumn{4}{|c|}{ Rußbildung } \\
\hline$\tau$ & 1 bis 1000 & $\mu \mathrm{s}$ & Induktionszeit, siehe Abb. (3.2) auf S. 17 \\
\hline$E_{\text {Ind }}$ & +220 & $\mathrm{~kJ} / \mathrm{mol}$ & Aktivierungsenergie Induktionszeit \\
\hline$k_{f}$ & 0,5 bis 50 & $10^{3} / \mathrm{s}$ & $\begin{array}{l}\text { Formale Rußbildungsgeschwindigkeits- } \\
\text { konstante, siehe Gl. (3.3) auf Seite } 16\end{array}$ \\
\hline$T_{\max }^{k_{f}}$ & $1950 \pm 50$ & $\mathrm{~K}$ & Maximumtemperatur von $k_{f}$ \\
\hline$E_{k f}$ & $+205-93$ & $\mathrm{~kJ} / \mathrm{mol}$ & Aktivierungsenergie unter und über $T_{\max }^{k_{f}}$ \\
\hline$f_{v}$ & 0,1 und 20 & $10^{-6}$ & $\begin{array}{l}\text { Untere und obere Rußvolumenbruch- } \\
\text { Nachweisgrenze, siehe Gl. (3.2) auf S. } 16\end{array}$ \\
\hline$\dot{f}_{v}$ & $10^{-4}$ bis 1 & $1 / \mathrm{s}$ & Zeitliche Änderung von $f_{v}$ \\
\hline$R A$ & 1 bis 100 & $\%$ & Rußausbeute, siehe Gl. (3.1) auf Seite 15 \\
\hline$T_{\max }^{R A}$ & $1900 \pm 100$ & $\mathrm{~K}$ & Maximumtemperatur der Rußausbeute \\
\hline$n$ & 0 bis 2 & - & Exponent der Kohlenstoffdichte $[C]^{n}$ \\
\hline$\Delta_{R} H_{12}$ & $1000 \pm 200$ & $\mathrm{~kJ} / \mathrm{mol}$ & $\begin{array}{l}\text { Reaktionsenthalpie vom Edukt zum Zwi- } \\
\text { schenprodukt }\end{array}$ \\
\hline$\Delta_{R} H_{23}$ & $-400 \pm 200$ & $\mathrm{~kJ} / \mathrm{mol}$ & $\begin{array}{l}\text { Reaktionsenthalpie vom Zwischenprodukt } \\
\text { zu Ruß }\end{array}$ \\
\hline \multicolumn{4}{|c|}{ Koagulation } \\
\hline$N_{0}$ & $2 \cdot 10^{19}$ & $1 / \mathrm{m}^{3}$ & Partikel-Anzahldichte zur Zeit 0 \\
\hline$N_{\infty}$ & $10^{17}$ bis $10^{19}$ & $1 / \mathrm{m}^{3}$ & Partikel-Anzahldichte zur Zeit $t$ \\
\hline$k_{\text {koag }}$ & $5 \cdot 10^{-16}$ & $\mathrm{~m}^{3} / \mathrm{s}$ & Geschwindigkeitskonstante, Gl. (4.2) S. 58 \\
\hline$K n$ & 0,1 bis 100 & - & KNUDSEN-Zahl, siehe Gl. (4.4) auf S. 59 \\
\hline$\tau_{\text {koag }}$ & 0,1 bis 10 & $\mathrm{~ms}$ & Halbwertszeit \\
\hline \multicolumn{4}{|c|}{ Sonstiges } \\
\hline$R$ & 8,314510 & $\mathrm{~J} /(\operatorname{mol~K})$ & Gaskonstante \\
\hline$M_{C}$ & 12 & $\mathrm{~g} / \mathrm{mol}$ & Molmasse des Kohlenstoffs \\
\hline$L$ & 70,0 & $\mathrm{~mm}$ & $\begin{array}{l}\text { Stoßrohrdurchmesser und einfache opti- } \\
\text { sche Weglänge, siehe Abb. (2.2) auf S. } 6\end{array}$ \\
\hline$\lambda$ & 632,8 & $\mathrm{~nm}$ & Lichtwellenlänge des HeNe-Lasers \\
\hline
\end{tabular}

\subsection{Berechnung von Stoßwellenparametern}

Temperatur- und Druck-Verhältnisse im Stoßrohr (Abbildung 5.1 und 5.2), Fortpflanzungsgeschwindigkeiten der Wellen und der Mediengrenze (Abbildung 5.3 und 5.4), sowie Zeiten und Orte des Zusammentreffen der Wellen (Abbildung 5.5 und 5.6), als Funktion der Stoßmachzahl $M$ berechnet nach Oertel [6]. 


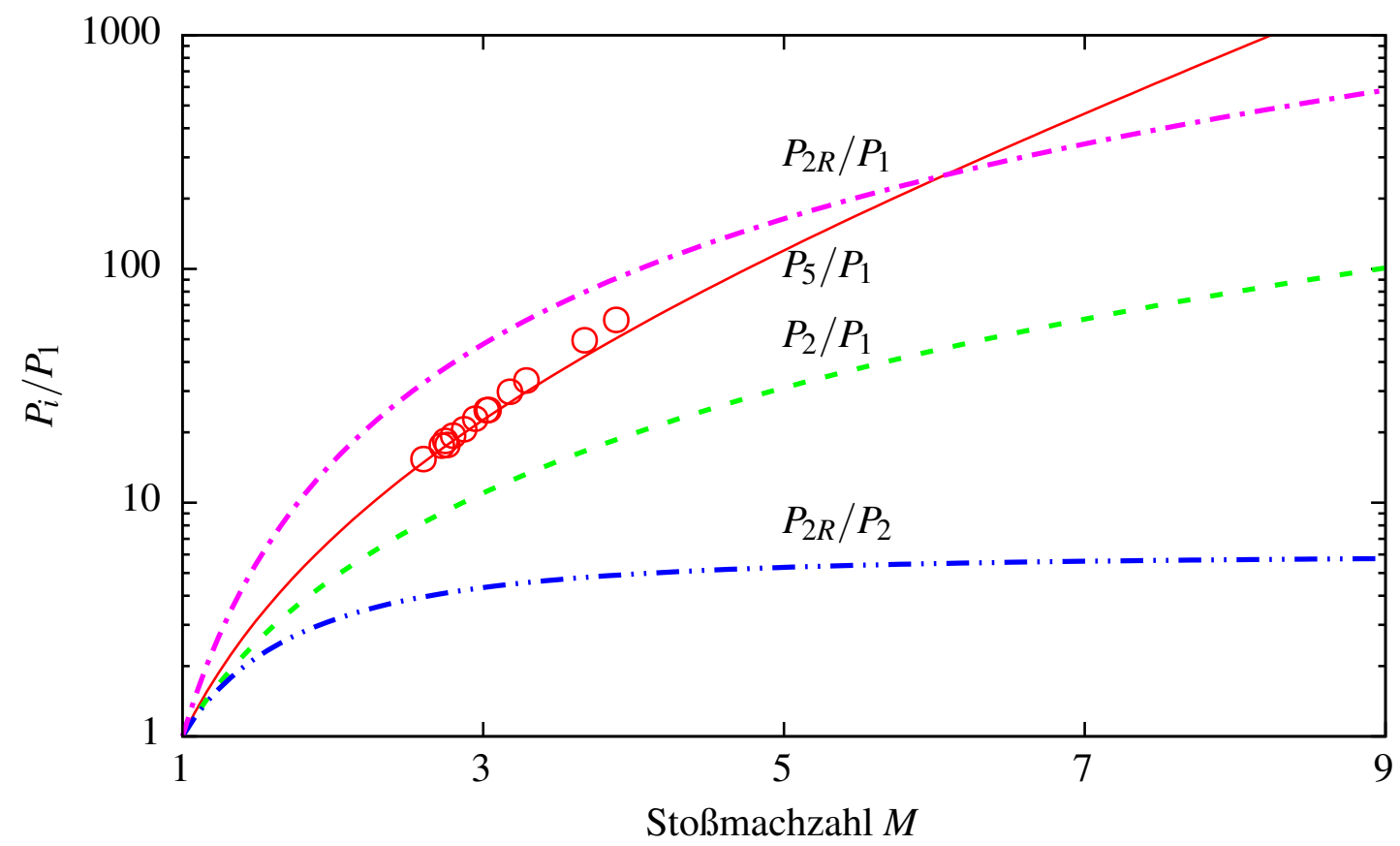

Abbildung 5.1: Darstellung des auf den Testgasdruck $P_{1}$ normierten Drucks im Treibgas $P_{5}$, hinter einfallender Stoßwelle $P_{2}$ und hinter reflektierter Stoßwelle $P_{2 R}$ als Funktion der Stoßmachzahl $M$. Treibgas $\mathrm{H}_{2}$, Laufgas Ar. Zusätzlich sind gemessene Treibgasdrücke $P_{5}$ im Bereich zwischen 2,5 und 4,0 MACH dargestellt.

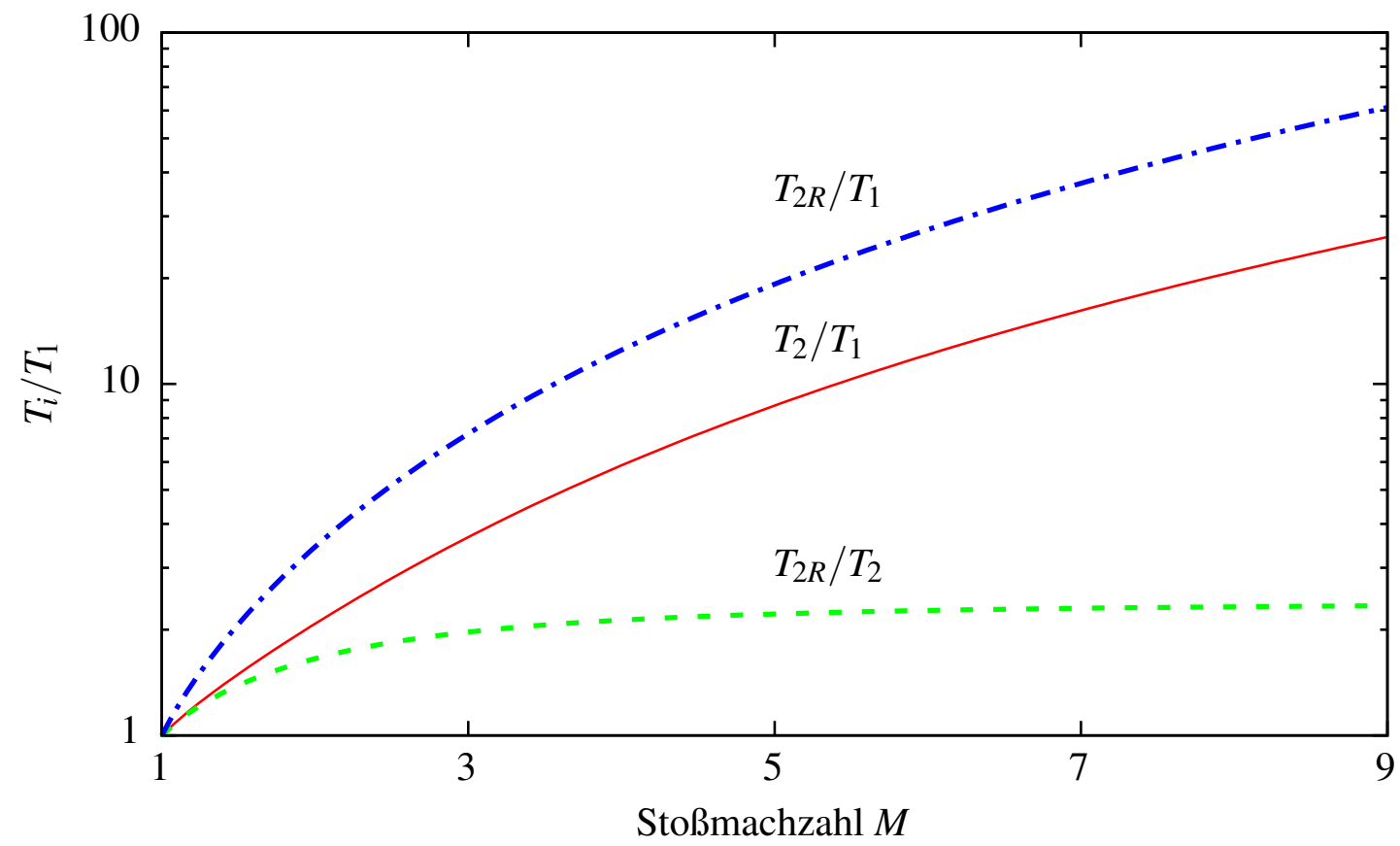

Abbildung 5.2: Darstellung der auf der Testgastemperatur $T_{1}$ normierten Temperatur hinter einfallender Stoßwelle $T_{2}$ und hinter reflektierter Stoßwelle $T_{2 R}$ als Funktion der Stoßmachzahl $M$. Treibgas $\mathrm{H}_{2}$, Laufgas Ar. 


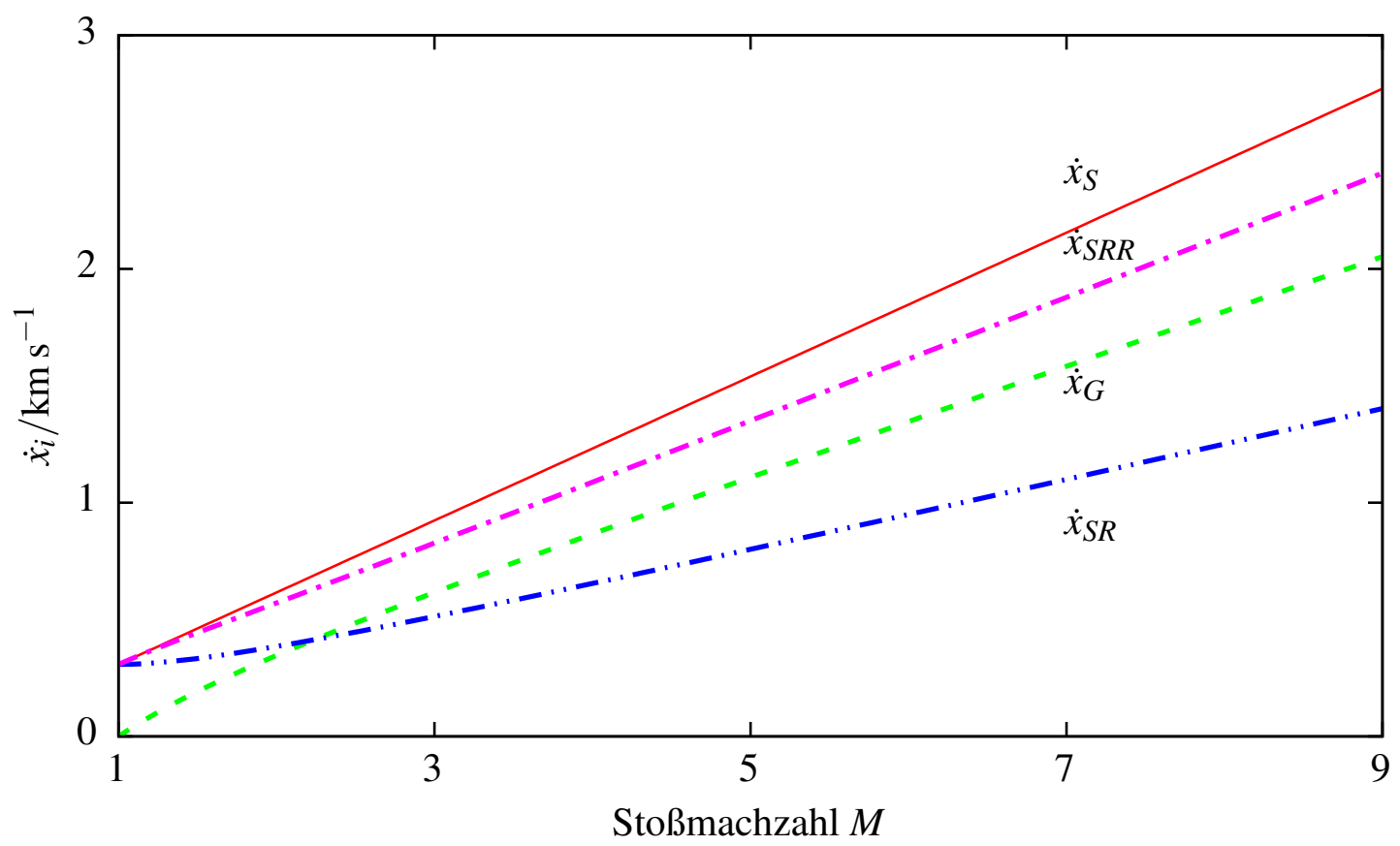

Abbildung 5.3: Die Fortpflanzungsgeschwindigkeiten der einfallenden Stoßwelle $\dot{x}_{S}$, der reflektierten Stoßwelle $\dot{x}_{S R}$, der Mediengrenze $\left(\dot{x}_{G}\right)$ und der an der Mediengrenze reflektierten Stoßwelle $\dot{x}_{S R R}$ als Funktion der Stoßmachzahl $M$. Treibgas $\mathrm{H}_{2}$, Laufgas Ar.

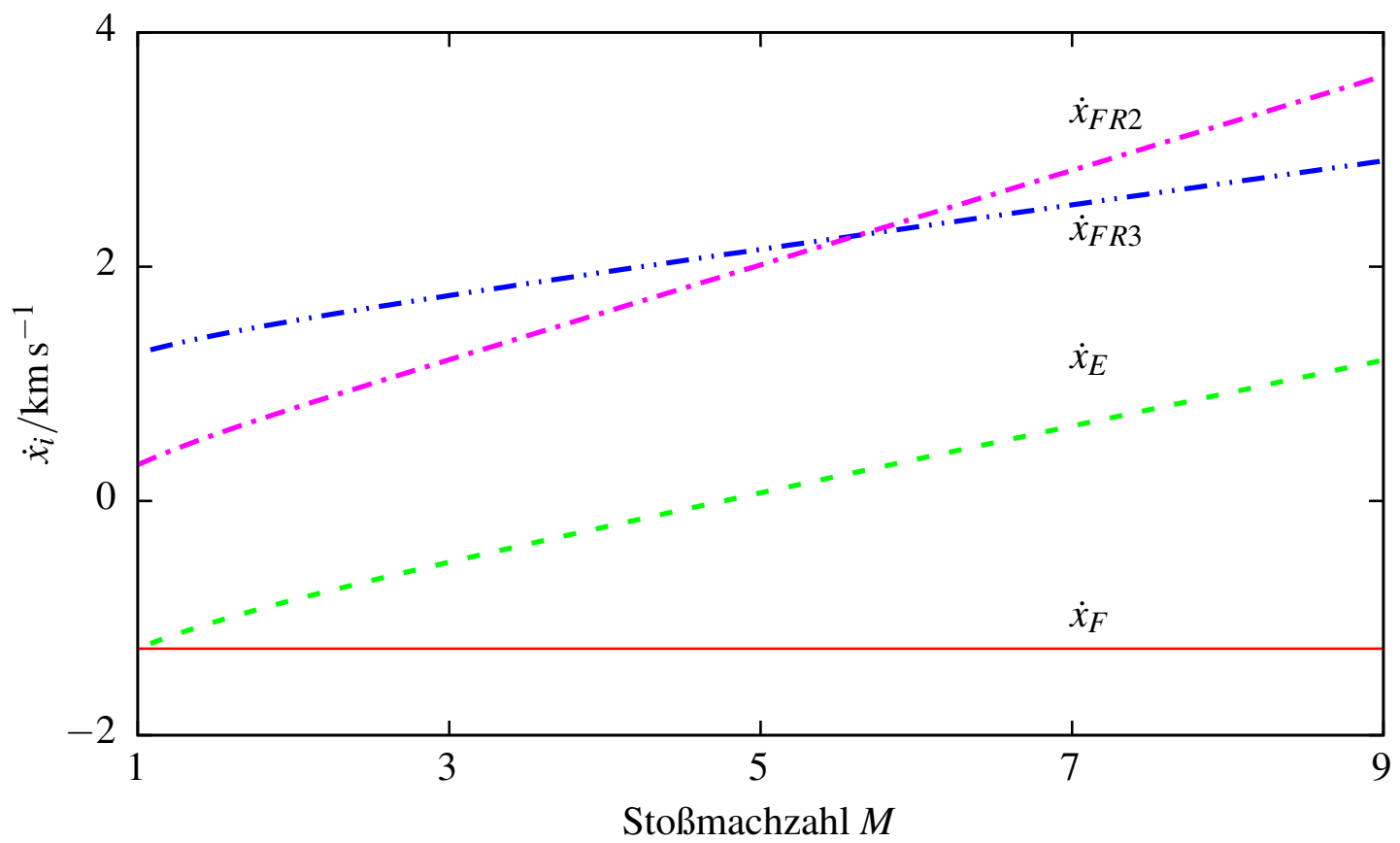

Abbildung 5.4: Die Fortpflanzungsgeschwindigkeiten der Front der Verdünnungswelle $\dot{x}_{F}$, des Endes der Verdünnungswelle $\dot{x}_{E}$ und der am Treibrohrende reflektierten Verdünnungswelle vor und hinter der Mediengrenze $\dot{x}_{F R 2}$ bzw. $\dot{x}_{F R 3}$ als Funktion der Stoßmachzahl $M$. Treibgas $\mathrm{H}_{2}$, Laufgas Ar. 


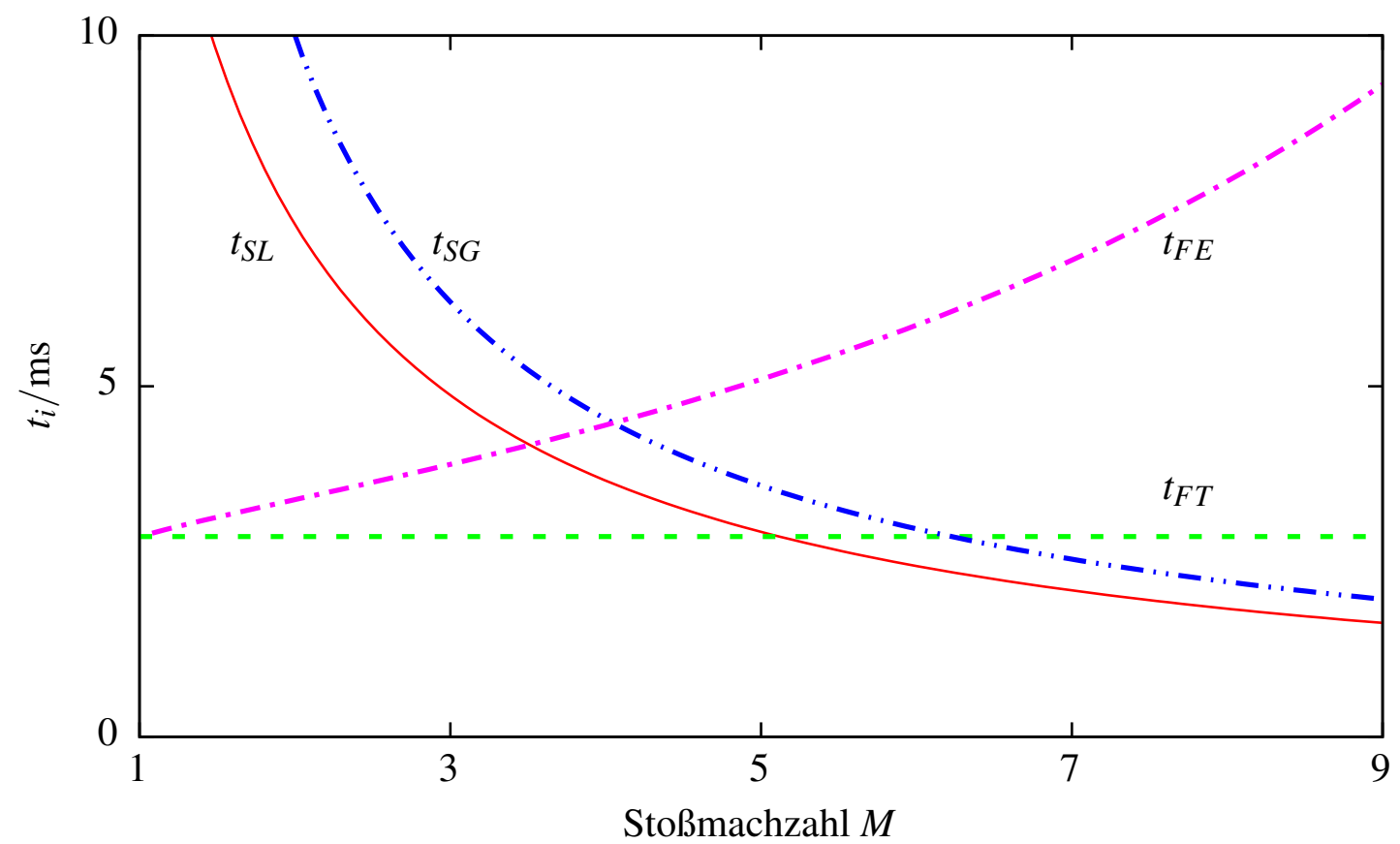

Abbildung 5.5: Die Zeiten des Eintreffens der Stoßwelle $t_{S L}$ (Verdünnungswelle $t_{F T}$ ) am Ende des Laufrohres (Treibrohres), des Zusammentreffen der Stoßwelle mit der Mediengrenze $t_{S G}$ und des Zusammentreffen der Front der Verdünnungswelle mit dem Ende der Verdünnungswelle $t_{F E}$ als Funktion der Stoßmachzahl $M$. Treibgas $\mathrm{H}_{2}$, Laufgas Ar.

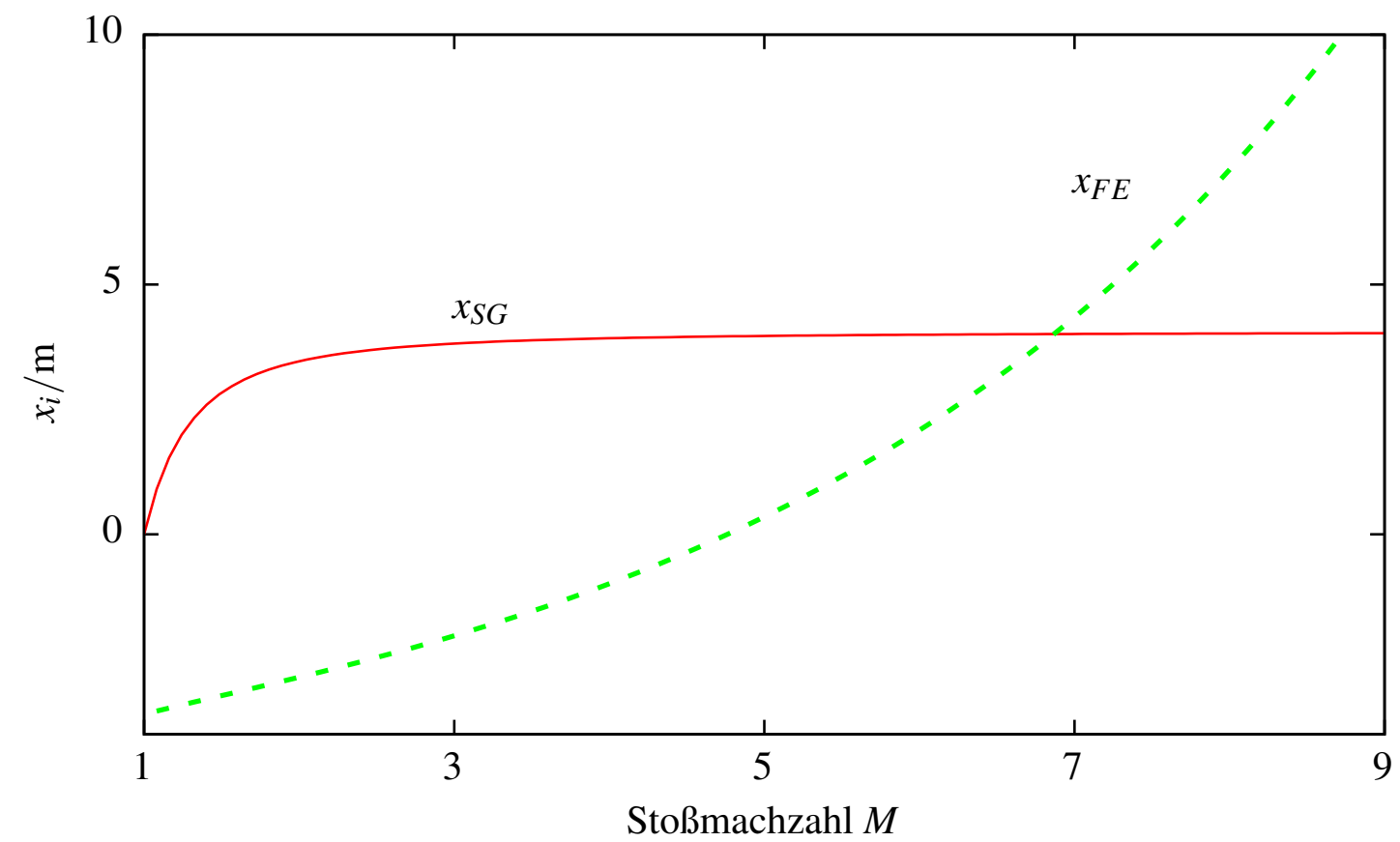

Abbildung 5.6: Die Entfernungen der Mediengrenze $x_{S G}$ und des Endes der Verdünnungswelle $x_{F E}$ von der Membran des Hochdruckrohres zum Zeitpunkt des Zusammentreffens mit der reflektierten Stoßwelle bzw. reflektierten Verdünnungswelle als Funktion der Stoßmachzahl $M$. Treibgas $\mathrm{H}_{2}$, Laufgas Ar. 


\subsection{Zusammenfassung}

In der vorliegenden Arbeit wurde die Rußbildung in der Kohlenwasserstoffpyrolyse hinter einfallenden und reflektierten Stoßwellen mit hoher Zeitauflösung absorptionsspektroskopisch beobachtet. Parallel dazu wurden am Ende der Rußbildung Ruß- und Gasproben durch ein besonders schnelles Ventilsystem gesammelt. Die Gasproben wurden mit GC und HPLC auf Pyrolyseprodukte analysiert. Die Struktur der Rußproben wurde mit Elektronenmikroskopen untersucht.

Im Temperaturbereich zwischen 1500 und $2500 \mathrm{~K}$ wurden Benzol, Phenylacetylen, Acetylen, Ethylen, n-Hexan und Methan pyrolysiert. Dabei wurde die Kohlenstoffdichte bis zu zwei Größenordnungen von 0,4 bis $40 \mathrm{~mol} / \mathrm{m}^{3}$ und der Druck zwischen 0,5 und $25 \mathrm{MPa}$ variiert. Außerdem wurde der Einfluß von geringen Mengen Eisenpentacarbonyl auf die Rußbildung in der Ethylenpyrolyse untersucht.

Vom Beginn der Pyrolyse bis zum Einsetzen der Rußbildung wurde stets eine Induktionszeit $\tau$ beobachtet, deren Länge von der Temperatur, der Kohlenstoffkonzentration und der Struktur des pyrolysierten Kohlenwasserstoffs abhängt. Dieser Zusammenhang wird mit einem Arrhenius-Ansatz beschrieben. Der Vorfaktor $A$ ist für Aromaten eine Größenordnung kleiner als für Alkane. Die scheinbare Aktivierungsenergie beträgt $(220 \pm 10) \mathrm{kJ} / \mathrm{mol}$. Die Induktionszeit der Rußbildung hängt für die untersuchten Alkane und Ethylen nur wenig von der Kohlenstoffdichte $a b, n<0,4$. In der Acetylen- und Benzolpyrolyse wurde für $n$ ein bester Wert von 0,75 gefunden.

$$
\tau=A \cdot \exp \left(E_{\text {Ind }} / R T\right) \cdot \frac{P^{0}}{[C]^{n}}
$$

Das Rußmassenwachstum, das unmittelbar nach der Induktionsperiode stattfindet, wurde mit einem Gesetz erster Ordnung beschrieben. $R A$ ist die Rußausbeute und $k_{f}$ die formale Rußbildungsgeschwindigkeitskonstante. Bis auf die Benzolpyrolyse zeigt $k_{f}$ in allen Kohlenwasserstoffpyrolysen ein nichtlineares Arrheniusverhalten mit einem Maximum bei $(1950 \pm 50) \mathrm{K}$. Durch Normierung auf die Kohlenstoffdichte $\left(k_{f} /[C]\right)$ wurde gezeigt, daß das Rußmassenwachstum in Pyrolysen und in vorgemischten Ethylenflammen, d. h. in einer sauerstoffhaltigen chemischen Umgebung, vergleichbar schnell abläuft.

$$
\frac{\mathrm{d}(R A)}{\mathrm{d} t}=k_{f} \cdot\left(R A_{\infty}-R A\right)
$$

Am Ende der Pyrolyse wurde eine konstant bleibende Rußmenge gebildet. Die Rußausbeute ist in der Pyrolyse der Aromaten und Acetylen bei $T=1800 \mathrm{~K}$ maximal. Für Ethylen und die Alkane liegt diese charakteristische Temperatur um 50 bzw. $150 \mathrm{~K}$ höher. Die Temperaturabhängigkeit der Rußausbeuten wurde quantitativ mit einem Drei-Spezies-Modell beschrieben. Eine Druckabhängigkeit der Rußausbeute wurde nur für Ethylen und Benzol gefunden. Die Temperaturabhängigkeit der Acetylenausbeute in Kohlenwasserstoffpyrolysen verhält sich gegenläufig zur Rußausbeute. Bei der Temperatur bei der die Rußausbeute ein Maximum hat, zeigt die Acetylenausbeute ein Minimum. Neben Ruß und Wasserstoff waren Acetylen gefolgt von Methan und Ethylen die wichtigsten Hauptprodukte. Die nachgewiesenen polycyclischen Aromaten trugen keine Seitengruppe und enthielten maximal einen Fünfring. Die Ausbeute an PAH war kleiner als ein tausendstel.

Der Zusatz von 200 ppm Eisenpentacarbonyl erhöhte die Rußausbeute in der Ethylenpyrolyse um ca. $30 \%$. Die Induktionszeit und die Massenwachstumskonstante wurden nicht beeinflußt. 
Der Durchmesser der primären Rußpartikel am Ende der Rußbildung lag unter allen Versuchsbedingungen im Bereich von $30 \mathrm{~nm}$. Daraus wurde gefolgert, daß die Haftwahrscheinlichkeit der Rußpartikel während der Koagulation mit zunehmender Kohlenstoffdichte von eins auf nahezu null abnimmt.

\subsection{Danksagung und Lebenslauf}

\section{Danksagung}

Meinem verehrten Lehrer Herrn Prof. Dr. H.Gg. Wagner danke ich herzlich für die Anregung und Förderung dieser Arbeit. Den Mitgliedern der Stoßwellen- und der Flammenabteilung insbesondere S. Bauerle, M. Tappe und Th. Thienel sowie den Gastwissenschaftlern Y. Karasevich (Moskau), St. Slavov (Sofia) und I. Zaslonko (Moskau), danke ich für ihre stete Unterstützung bei der Planung, Ausführung und Auswertung der Experimente und den fruchtbaren Diskussionen. Besonders bedanke ich mich bei den Mitarbeitern der Institutswerkstätten, die mir beim Aufbau der Apparaturen tatkräftig zur Seite standen. Der Gesellschaft für wissenschaftliche Datenverarbeitung Göttingen (GWDG) danke ich für die freundliche Bereitstellung von Geräten und Programmen.

\section{Lebenslauf}

Am 2. Mai 1961 wurde ich als erster Sohn der Eheleute Friedrich und Hanna Tanke in Walsrode geboren. Von 1967 bis 1981 besuchte ich die Schule, die ich mit der allgemeinen Hochschulreife abgeschlossen habe. Zum Wintersemester 1981/2 immatrikulierte ich mich an der Göttinger Georg-August-Universität im Fach Chemie (Diplom). Während einer längeren Studienpause arbeitete ich in einem Göttinger Couleur-, Heraldik- und Porzellanmalerei-Betrieb (Arnold'sche Manufaktur). Im April 1990 legte ich die Diplomvorprüfung ab. In der Zeit vom Februar bis September 1991 fertigte ich im Arbeitskreis von Prof. Dr. H.Gg. Wagner die Diplomarbeit über das Thema „Zeitaufgelöste Absorptionsmessungen bei der Rußbildung aus n-Hexan und Benzol hinter Hochdruck-Stoßwellen“ an. Seit der Diplomprüfung im Oktober 1991 bin ich im Institut für Physikalische Chemie beschäftigt. Neben der Betreuung von Lehrveranstaltungen und Praktika wurde mir Gelegenheit gegeben Fragestellungen der Verbrennungschemie in Theorie und Experiment zu untersuchen. Im Rahmen dieser Tätigkeit entstand die vorliegende Arbeit. 